\title{
HFIP solvent enables alcohols to act as alkylating agents in stereoselective heterocyclization
}

Yuxiang Zhu, Ignacio Colomer, Amber L. Thompson and Timothy J. Donohoe*

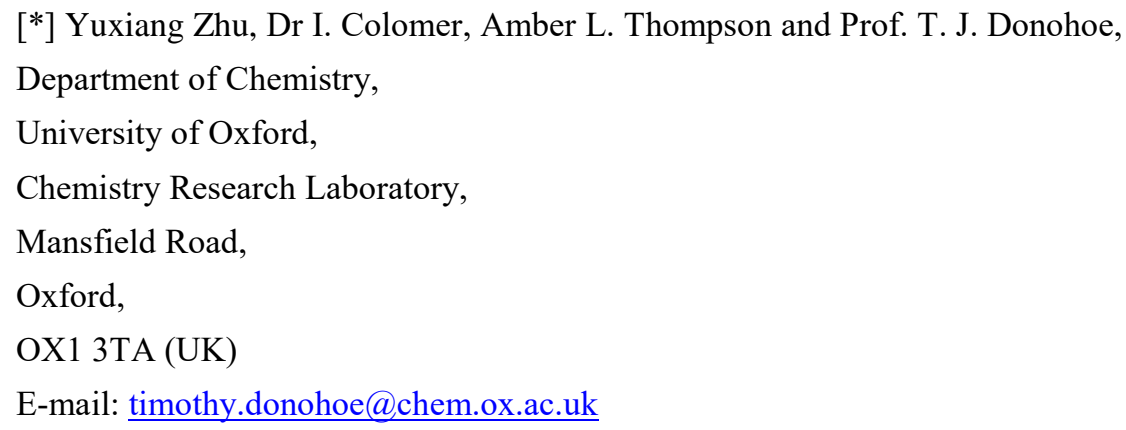




\section{Table of Contents}

Compound S11: ${ }^{1} \mathbf{H}$ NMR and ${ }^{13} \mathbf{C}$ NMR

Compound S12: ${ }^{1} \mathrm{H}$ NMR and ${ }^{13} \mathrm{C}$ NMR

S5

Compound S13: ${ }^{1} \mathrm{H}$ NMR and ${ }^{13} \mathrm{C}$ NMR

Compound $(E)-\mathbf{1 b}:{ }^{1} \mathbf{H}$ NMR and ${ }^{13} \mathrm{C}$ NMR

S7

Compound $(E)-1 c:{ }^{\mathbf{1}} \mathbf{H}$ NMR and ${ }^{13} \mathrm{C}$ NMR

S8

Compound 1f: ${ }^{\mathbf{1}} \mathbf{H}$ NMR and ${ }^{{ }^{13} \mathbf{C}} \mathbf{N M R}$

S9

Compound (E)-1h: ${ }^{\mathbf{1}} \mathrm{H}$ NMR and ${ }^{{ }^{13}} \mathrm{C}$ NMR

S10

Compound $(E)-1 \mathbf{1 i}:{ }^{\mathbf{1}} \mathrm{H}$ NMR and ${ }^{{ }^{13}} \mathrm{C}$ NMR

S11

Compound $(E)-\mathbf{1 j}$ : ${ }^{\mathbf{1}} \mathbf{H}$ NMR and ${ }^{{ }^{13}} \mathbf{C}$ NMR

$\mathrm{S} 12$

Compound $(E)-11:{ }^{1} \mathrm{H}$ NMR and ${ }^{13} \mathrm{C}$ NMR

S13

Compound $(E)-1 m:{ }^{1} \mathbf{H}$ NMR and ${ }^{13} \mathrm{C}$ NMR

S14

Compound 3a: ${ }^{\mathbf{1}} \mathbf{H}$ NMR and ${ }^{13} \mathbf{C}$ NMR

S15

Compound 3b: ${ }^{1} \mathrm{H}$ NMR, ${ }^{13} \mathrm{C}$ NMR, COSY, HSQC, HMBC and NOESY-2D

S16

Compound 3c: ${ }^{1} \mathrm{H}$ NMR, ${ }^{13} \mathrm{C}$ NMR, COSY, HSQC and NOESY-2D

S19

Compound 3d: ${ }^{1} \mathbf{H}$ NMR and ${ }^{13} \mathbf{C}$ NMR

S22

Compound 3e: ${ }^{1} \mathbf{H}$ NMR and ${ }^{13} \mathrm{C}$ NMR

S23

Compound 3f: ${ }^{\mathbf{1}} \mathbf{H}$ NMR and ${ }^{13} \mathbf{C}$ NMR

S24

Compound 3g: ${ }^{1} \mathbf{H}$ NMR and ${ }^{13} \mathbf{C}$ NMR

S25

Compound 3h: ${ }^{1} \mathbf{H}$ NMR and ${ }^{13} \mathbf{C}$ NMR

Compound 3i (diasteromers A and B): ${ }^{1} \mathrm{H}$ NMR, ${ }^{13} \mathrm{C}$ NMR, COSY, HSQC and NOESY-2D

Compound 3i (diasteromers $\mathrm{C}$ and D): ${ }^{\mathbf{1}} \mathrm{H}$ NMR and ${ }^{\mathbf{1}} \mathrm{C}$ NMR

Compound 3j: ${ }^{\mathbf{1}} \mathbf{H}$ NMR and ${ }^{\mathbf{1 3}} \mathbf{C}$ NMR

Compound 3k: ${ }^{\mathbf{1}} \mathrm{H}$ NMR and ${ }^{13} \mathbf{C}$ NMR

Compound 31: ${ }^{\mathbf{1}} \mathrm{H}$ NMR and ${ }^{13} \mathrm{C}$ NMR

Compound 3m: ${ }^{1} \mathrm{H}$ NMR, ${ }^{13} \mathrm{C}$ NMR, COSY, HSQC and NOESY-2D $\quad$ S34

Compound 3n: ${ }^{1} \mathbf{H}$ NMR and ${ }^{13} \mathbf{C}$ NMR

Compound 3o: ${ }^{1} \mathbf{H}$ NMR and ${ }^{13} \mathrm{C}$ NMR

Compound 3p: ${ }^{1} \mathrm{H}$ NMR and ${ }^{13} \mathrm{C}$ NMR

Compound 3q: ${ }^{1} \mathbf{H}$ NMR and ${ }^{13} \mathbf{C}$ NMR

Compound 3r: ${ }^{1} \mathbf{H}$ NMR and ${ }^{13} \mathrm{C}$ NMR

Compound 3s: ${ }^{1} \mathbf{H}$ NMR and ${ }^{13} \mathrm{C}$ NMR

Compound 3t: ${ }^{1} \mathbf{H}$ NMR and ${ }^{13} \mathbf{C}$ NMR

Compound 3u: ${ }^{1} \mathbf{H}$ NMR and ${ }^{13} \mathbf{C}$ NMR

Compound 3v: ${ }^{1} \mathbf{H}$ NMR and ${ }^{13} \mathbf{C}$ NMR

Compound 3w: ${ }^{1} \mathbf{H}$ NMR and ${ }^{13} \mathrm{C}$ NMR

Compound 3x: ${ }^{1} \mathrm{H}$ NMR, ${ }^{13} \mathrm{C}$ NMR, COSY, HSQC and NOESY-2D

Compound 3y: ${ }^{\mathbf{1}} \mathbf{H}$ NMR and ${ }^{\mathbf{1 3}} \mathbf{C}$ NMR

Compound 3z: ${ }^{1} \mathrm{H}$ NMR, ${ }^{13} \mathrm{C}$ NMR, COSY, HSQC and NOESY-2D

Compound 3aa: ${ }^{1} \mathrm{H}$ NMR, ${ }^{13} \mathrm{C}$ NMR, COSY, HSQC and NOESY-2D

Compound 3ab: ${ }^{\mathbf{1}} \mathbf{H}$ NMR and ${ }^{13} \mathbf{C}$ NMR

Compound 3ac: ${ }^{\mathbf{1}} \mathbf{H}$ NMR and ${ }^{13} \mathbf{C}$ NMR

Compound 3ad: ${ }^{\mathbf{1}} \mathbf{H}$ NMR and ${ }^{13} \mathbf{C}$ NMR 
${ }^{1} \mathrm{H}$ NMR (400 MHz, $\left.\mathrm{CDCl}_{3}\right)$
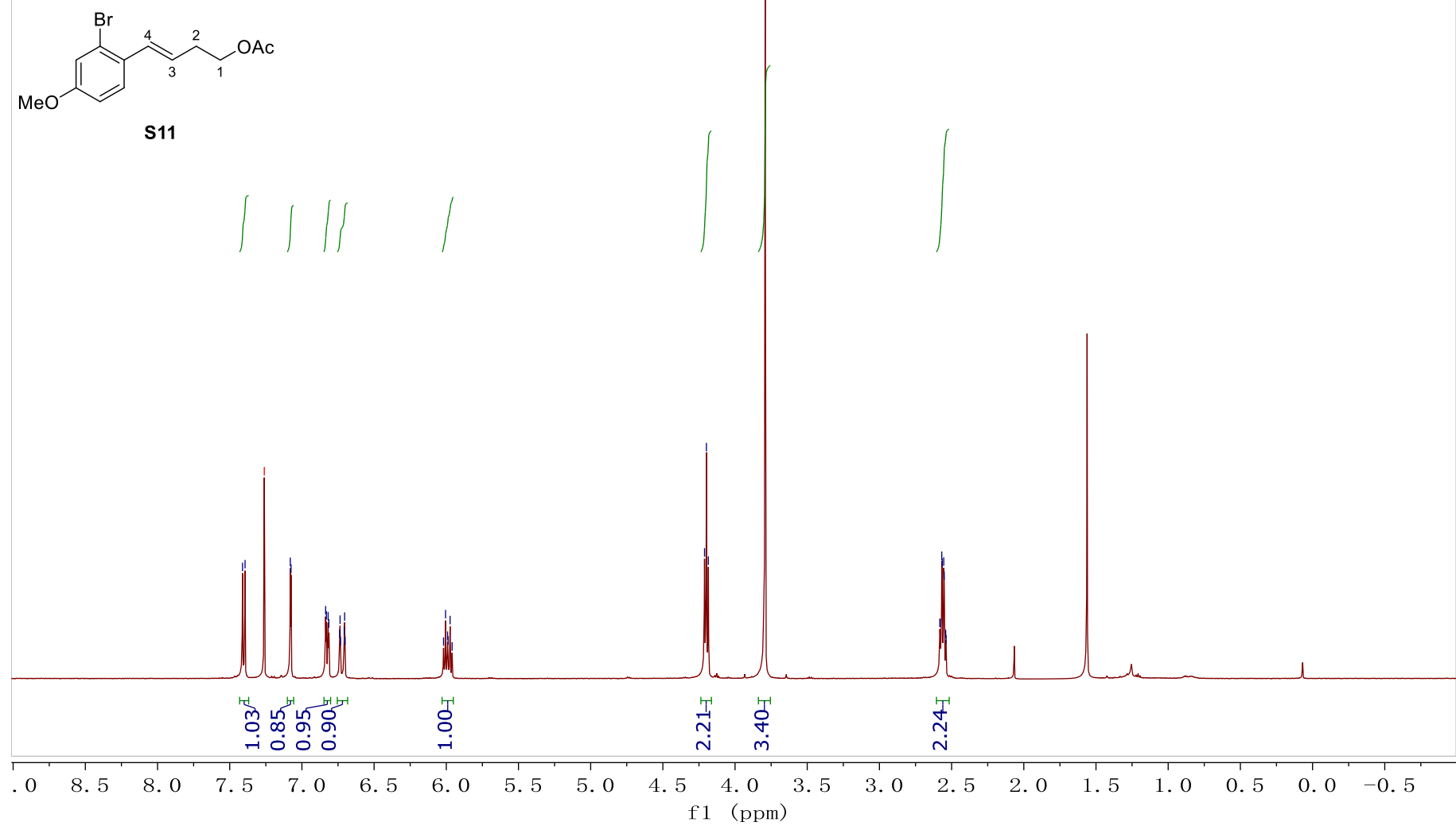

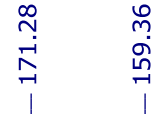

m.

${ }^{13} \mathrm{C}$ NMR (100 MHz, $\mathrm{CDCl}_{3}$ )<smiles>COc1ccc(/C=C/CC(C)OC(C)=O)c(Br)c1</smiles>

S11
-

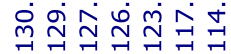

1 $7 \rightarrow 7$
ํㅡㄴ

₹ํㅡ

ヘำ

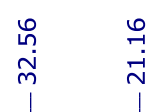
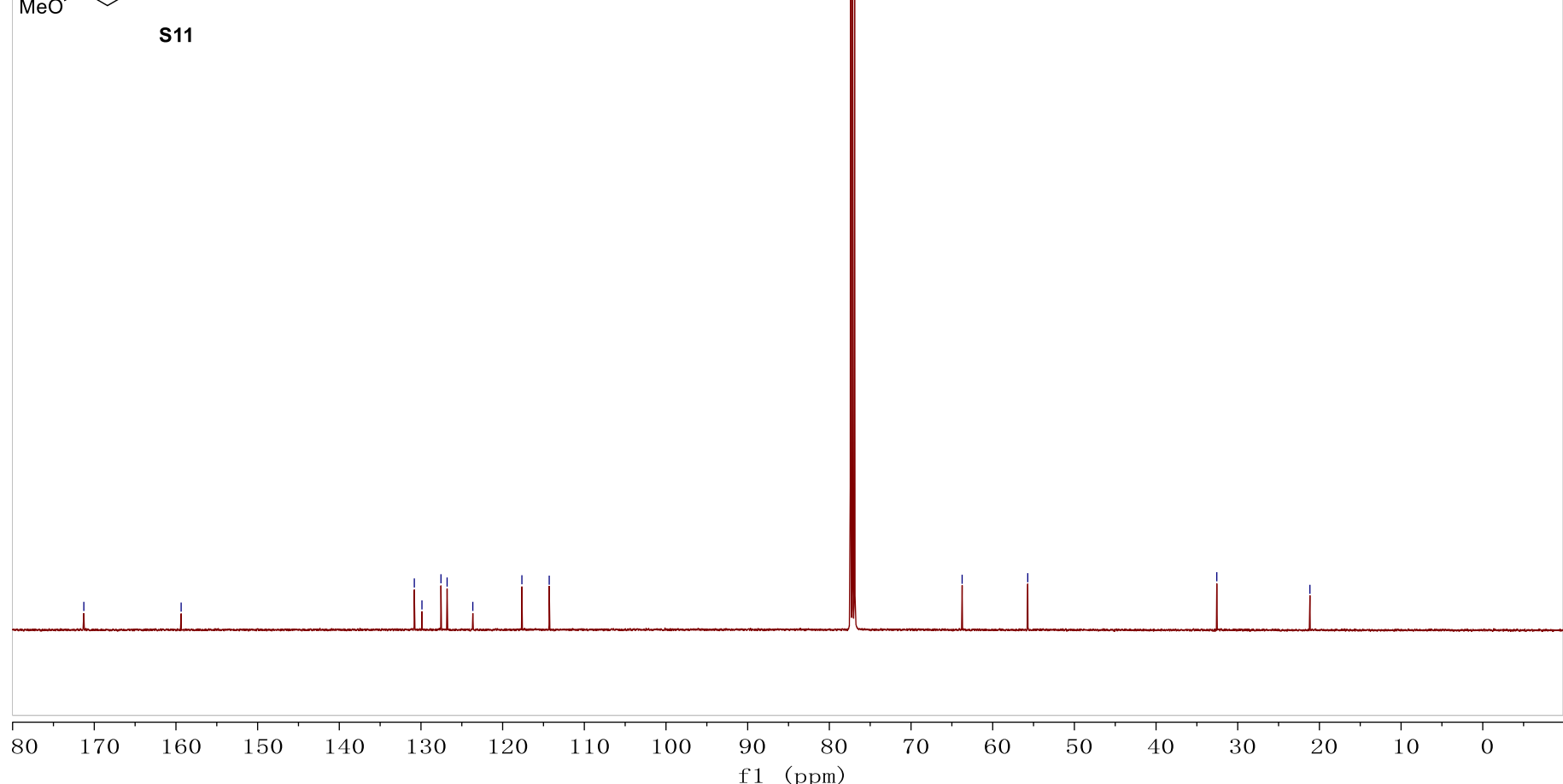
荇

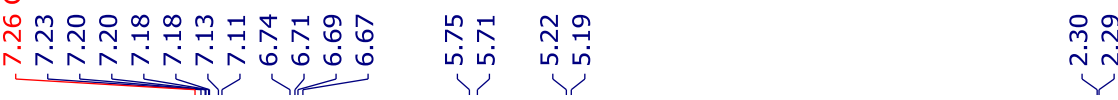

${ }^{1} \mathrm{H}$ NMR (400 MHz, $\left.\mathrm{CDCl}_{3}\right)$

$\overbrace{31}^{2} O A C$

S12

\section{$\stackrel{4}{i}$}

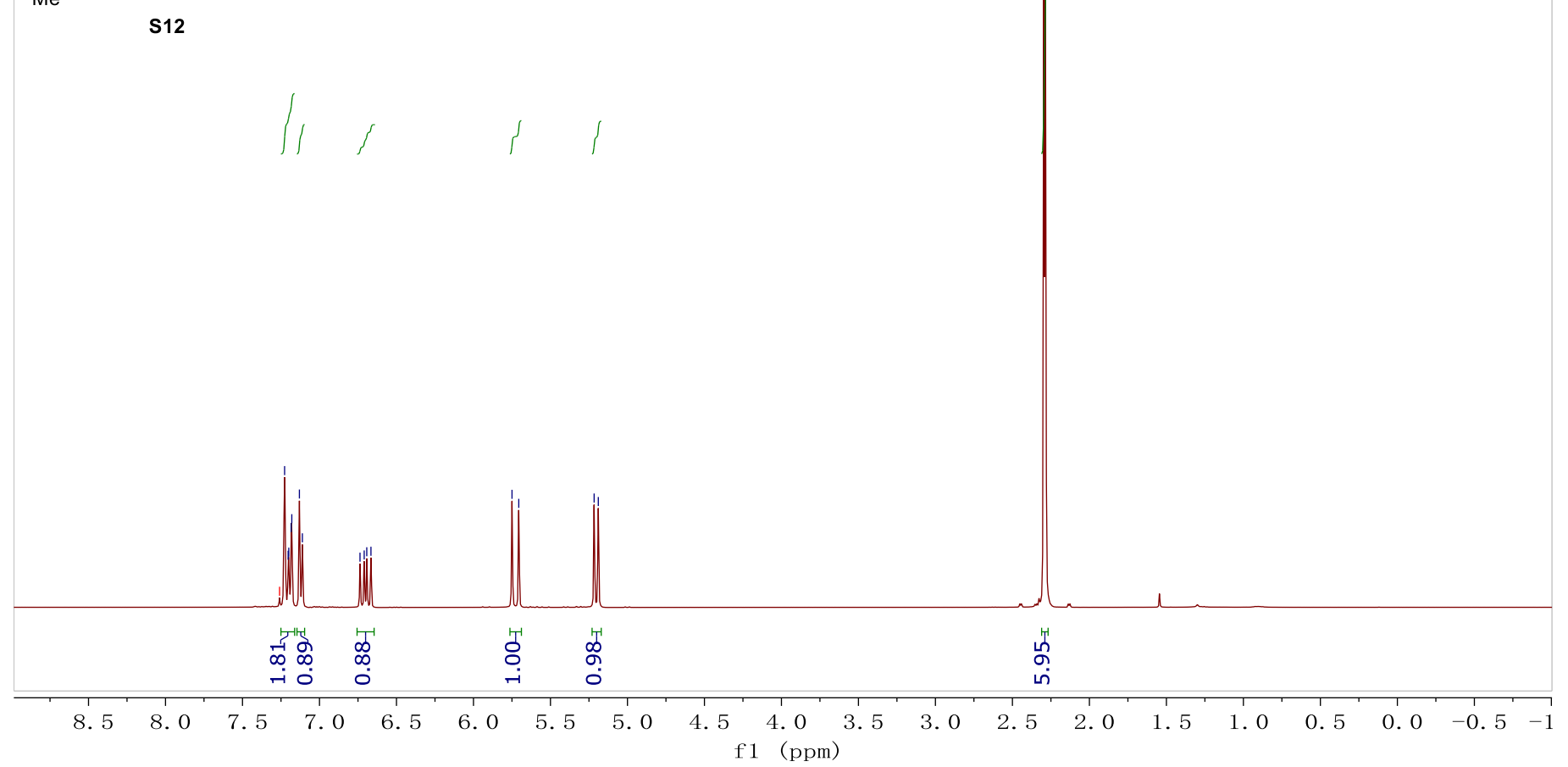

${ }^{13} \mathrm{C}$ NMR (100 MHz, $\left.\mathrm{CDCl}_{3}\right)$

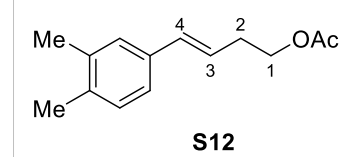

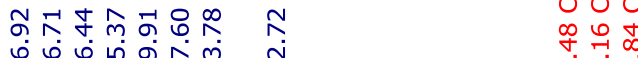

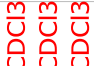

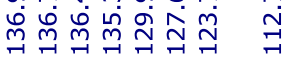

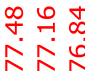

손

नَळ

คे

S12

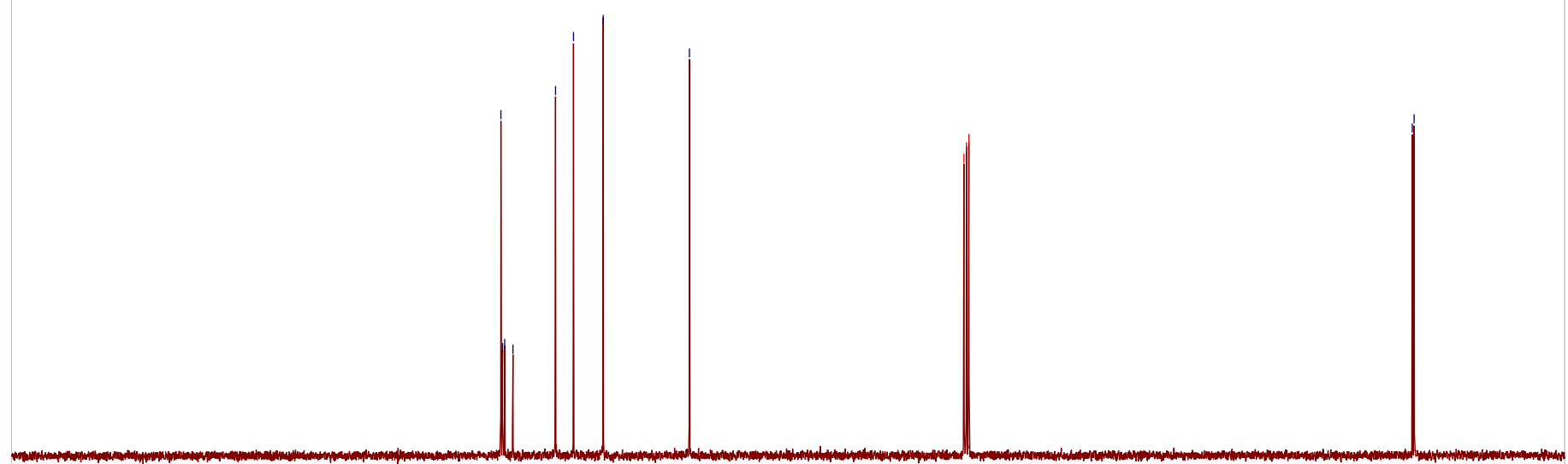




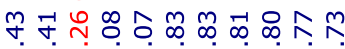

ヘヘヘベヘ

ำกำ

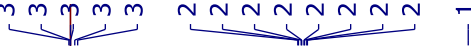

${ }^{1} \mathrm{H}$ NMR (400 MHz, $\mathrm{CDCl}_{3}$ )<smiles>COc1ccc(C=CCC(C)O)c(Br)c1</smiles>

$E-1 b$
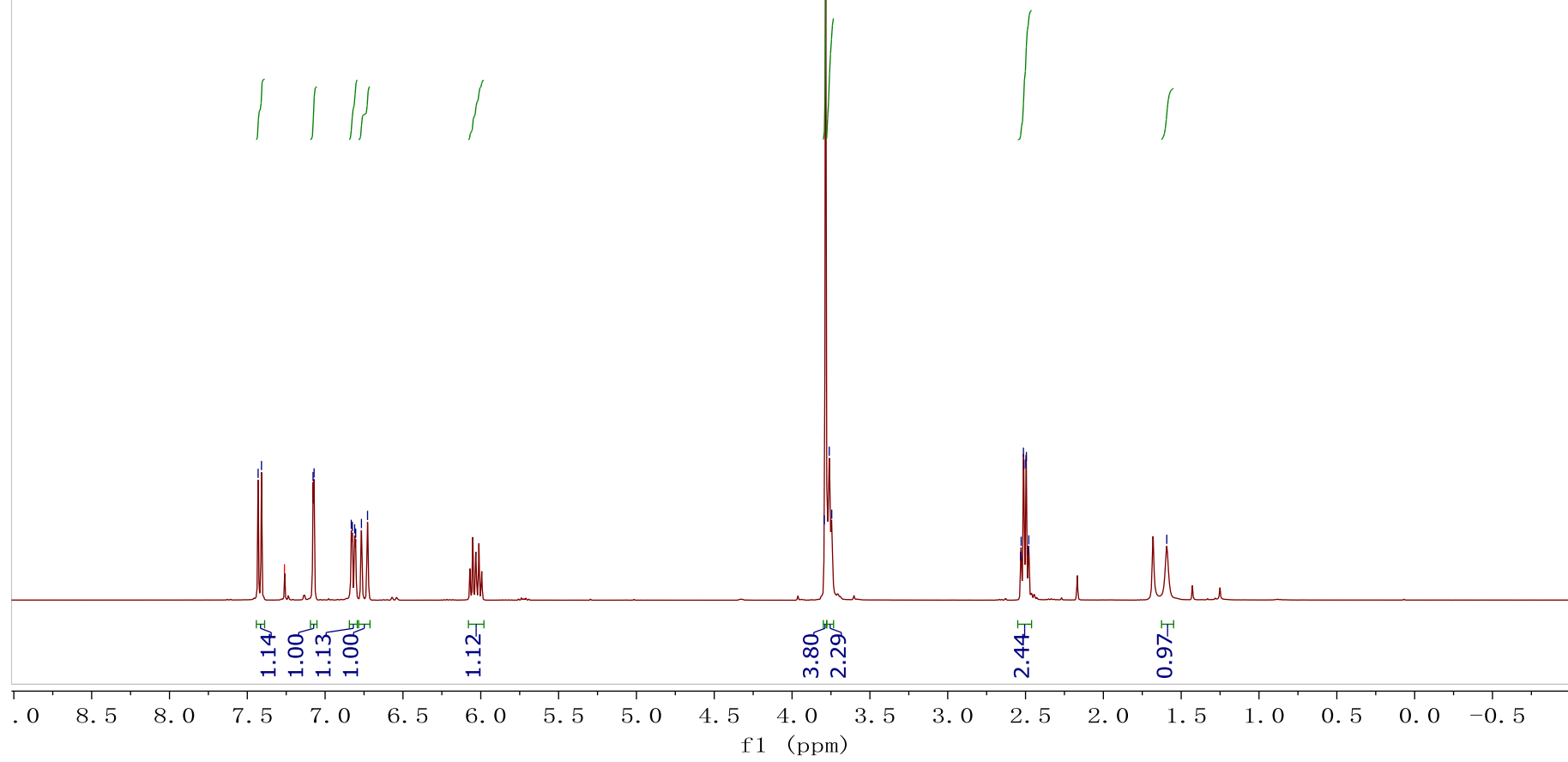

ํํำ

${ }^{13} \mathrm{C}$ NMR (100 MHz, $\left.\mathrm{CDCl}_{3}\right)$

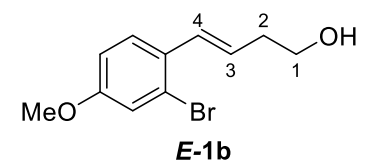

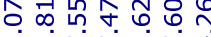

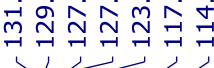

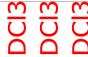

$\infty$ o

소

$E-1 b$

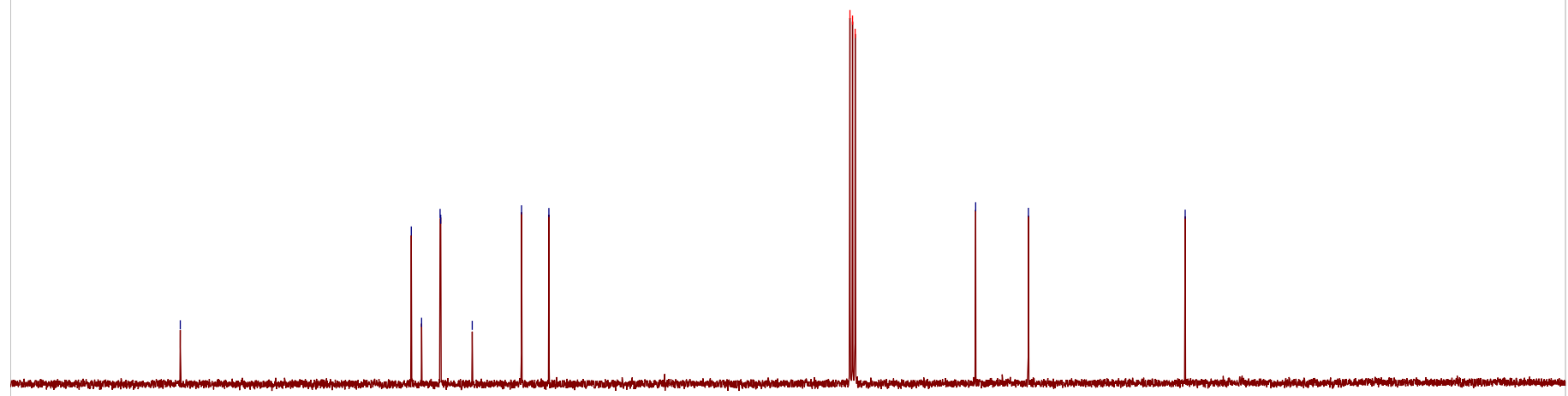


บั

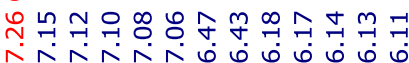

요송

$\underbrace{m i m}$

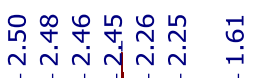

${ }^{1} \mathrm{H}$ NMR (400 MHz, $\left.\mathrm{CDCl}_{3}\right)$

$\overbrace{\mathrm{Me}=1 \mathrm{C}}^{4} \overbrace{1}^{2} \mathrm{OH}$

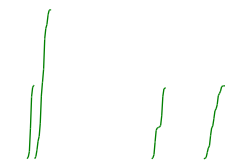

$\int$

in

ESc
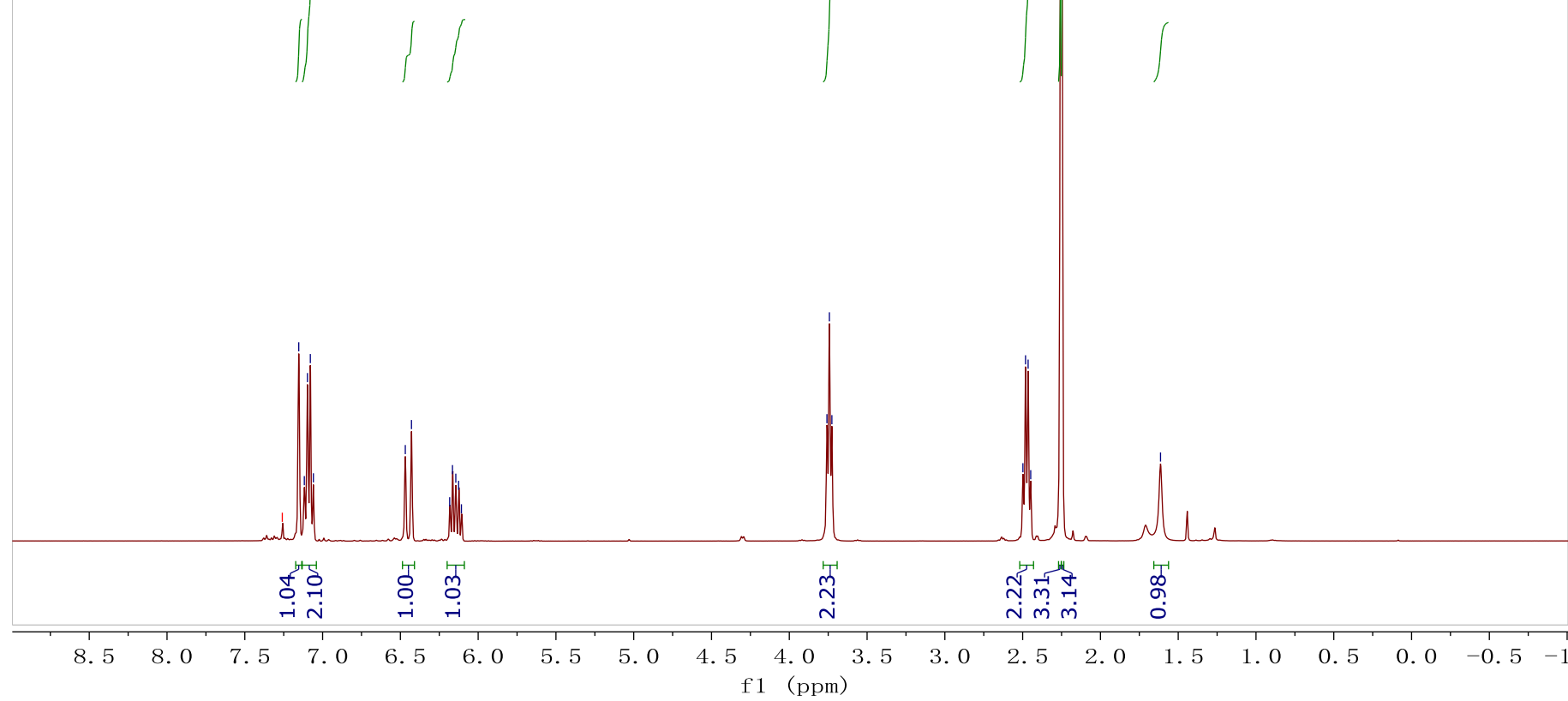

\section{${ }^{13} \mathrm{C}$ NMR (100 MHz, $\mathrm{CDCl}_{3}$ )}

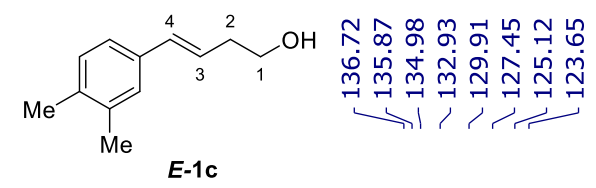

ํㅡㄴ $\frac{m}{\mathrm{U}} \frac{m}{\mathrm{U}}$

我桴

소용

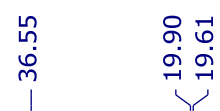

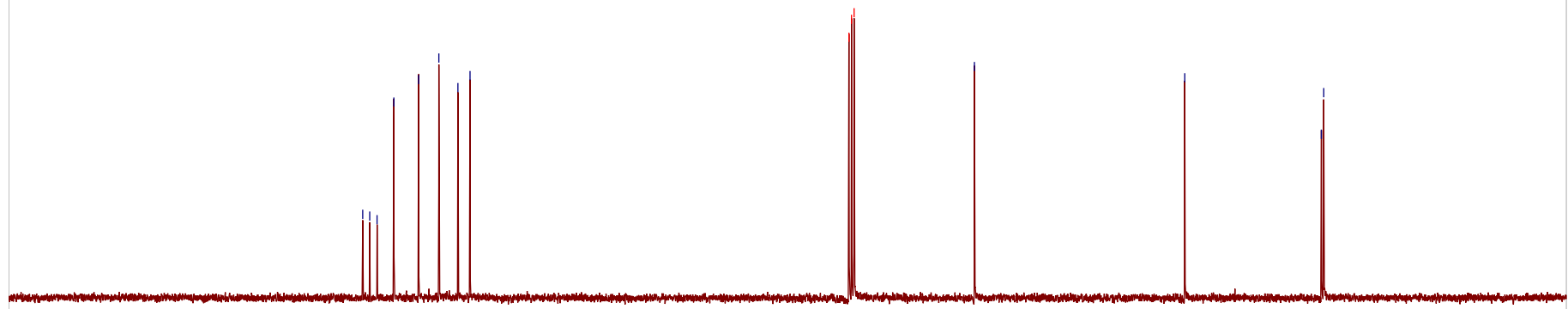


${ }^{1}$ H NMR (400 MHz, $\mathrm{CDCl}_{3}$ )

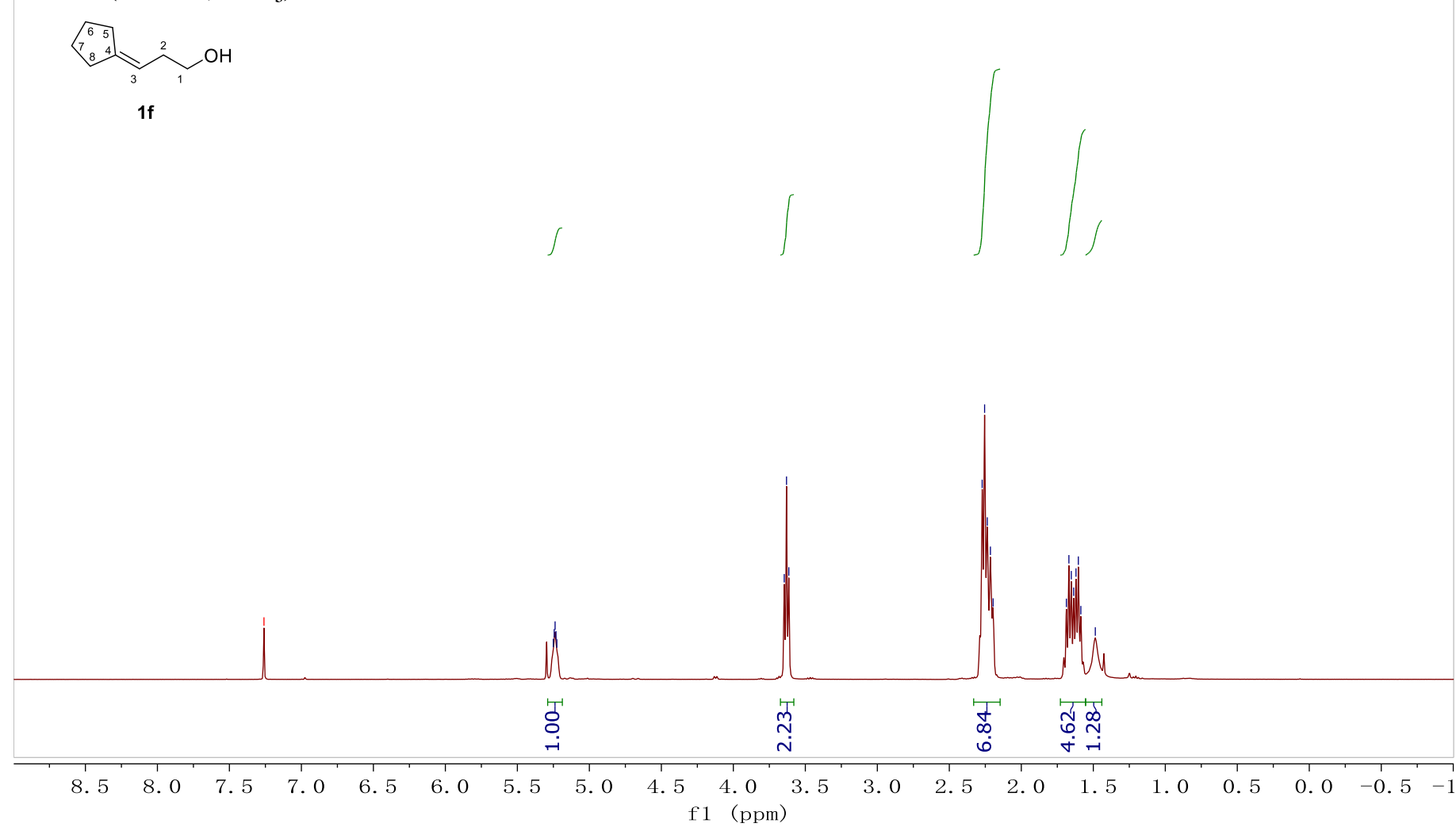

\section{${ }^{13} \mathrm{C}$ NMR (100 MHz, $\left.\mathrm{CDCl}_{3}\right)$}<smiles>CC(COCC(C)(C)C)CC1=CC=CC1</smiles>

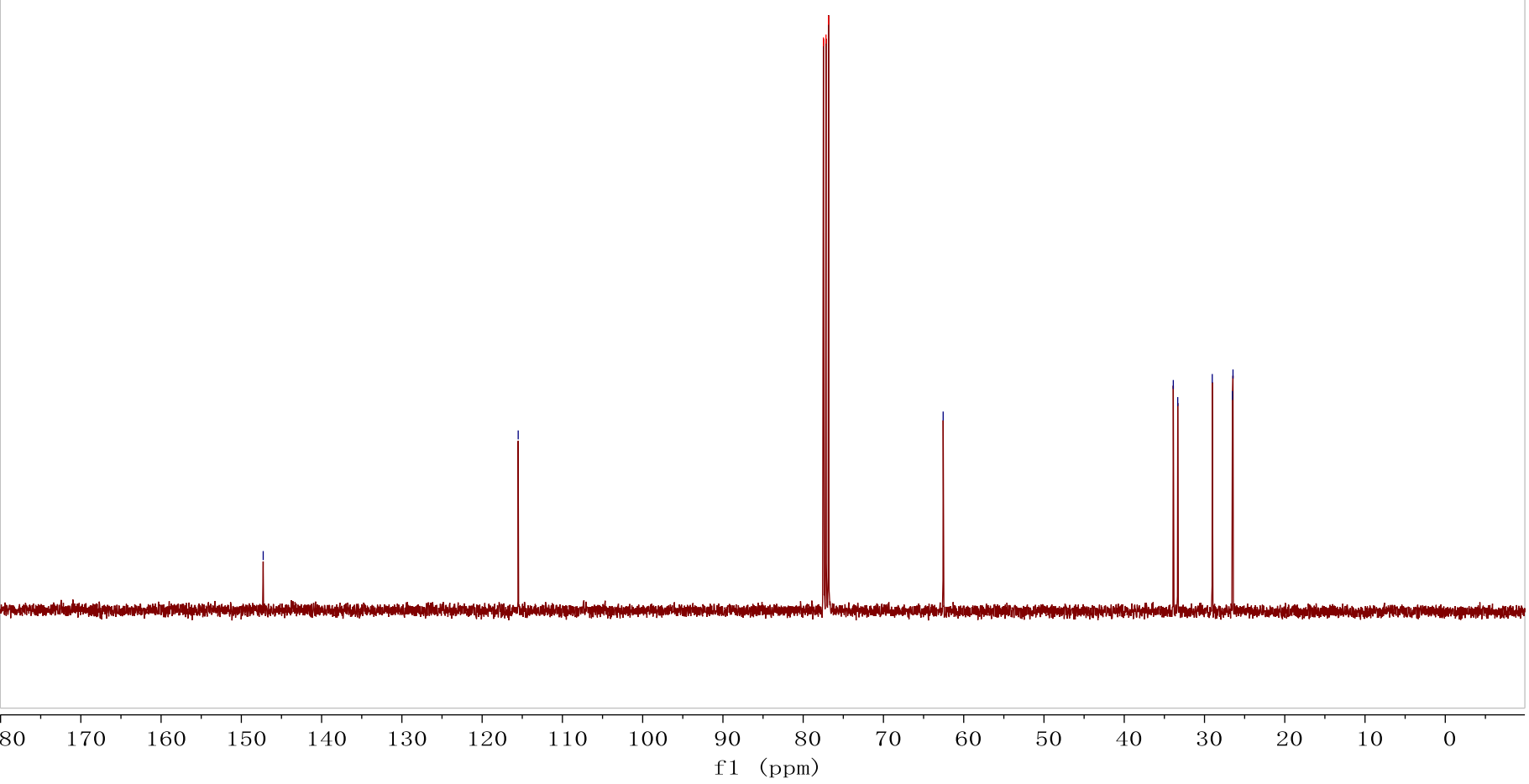


m

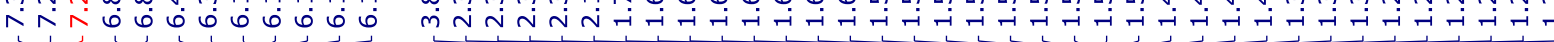

\section{${ }^{1} \mathrm{H}$ NMR (400 MHz, $\left.\mathrm{CDCl}_{3}\right)$}<smiles>COc1ccc(/C=C/Cc2ccccc2O)cc1</smiles>

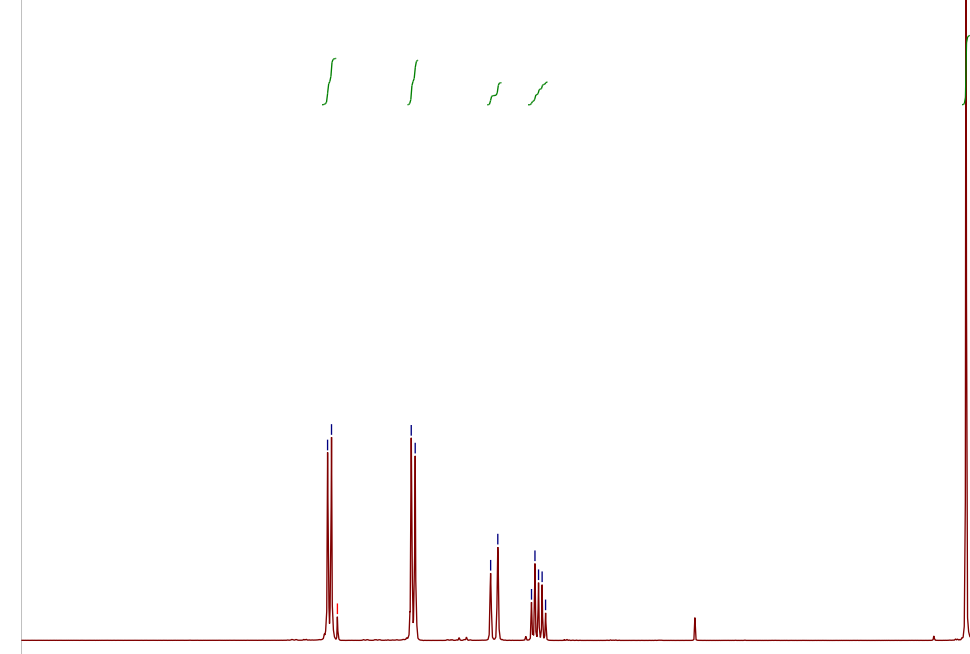

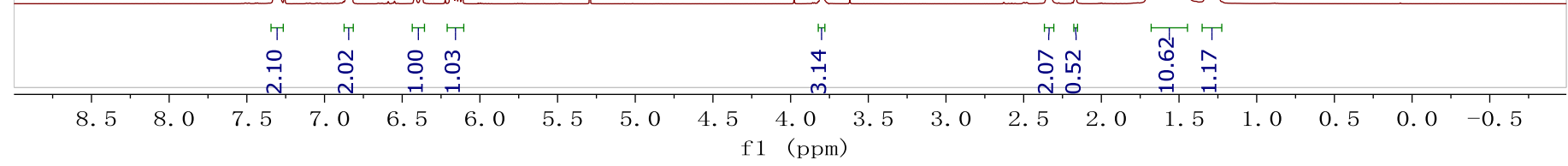

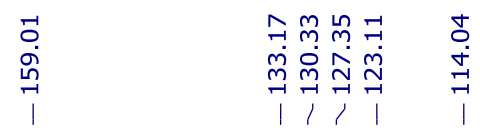
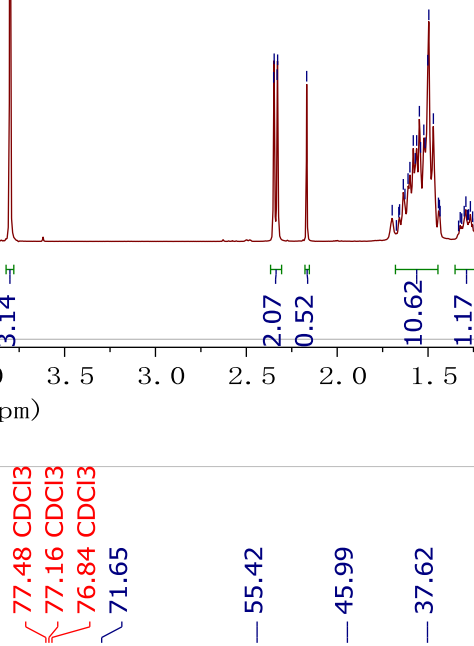

\section{${ }^{13} \mathrm{C}$ NMR (100 MHz, $\left.\mathrm{CDCl}_{3}\right)$}

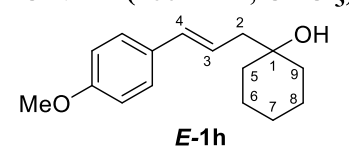

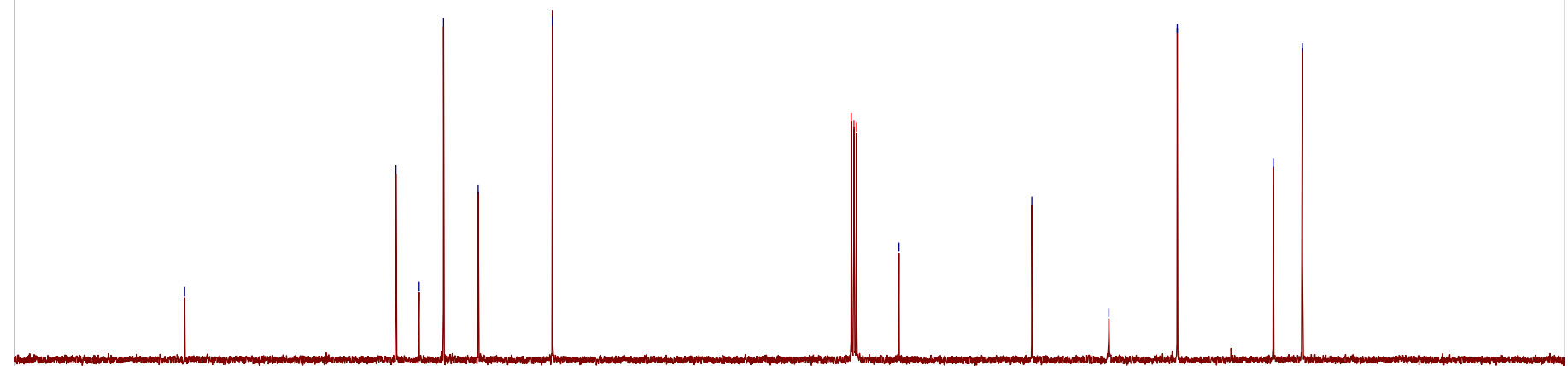


${ }^{1} \mathrm{H}$ NMR (400 MHz, $\left.\mathrm{CDCl}_{3}\right)$

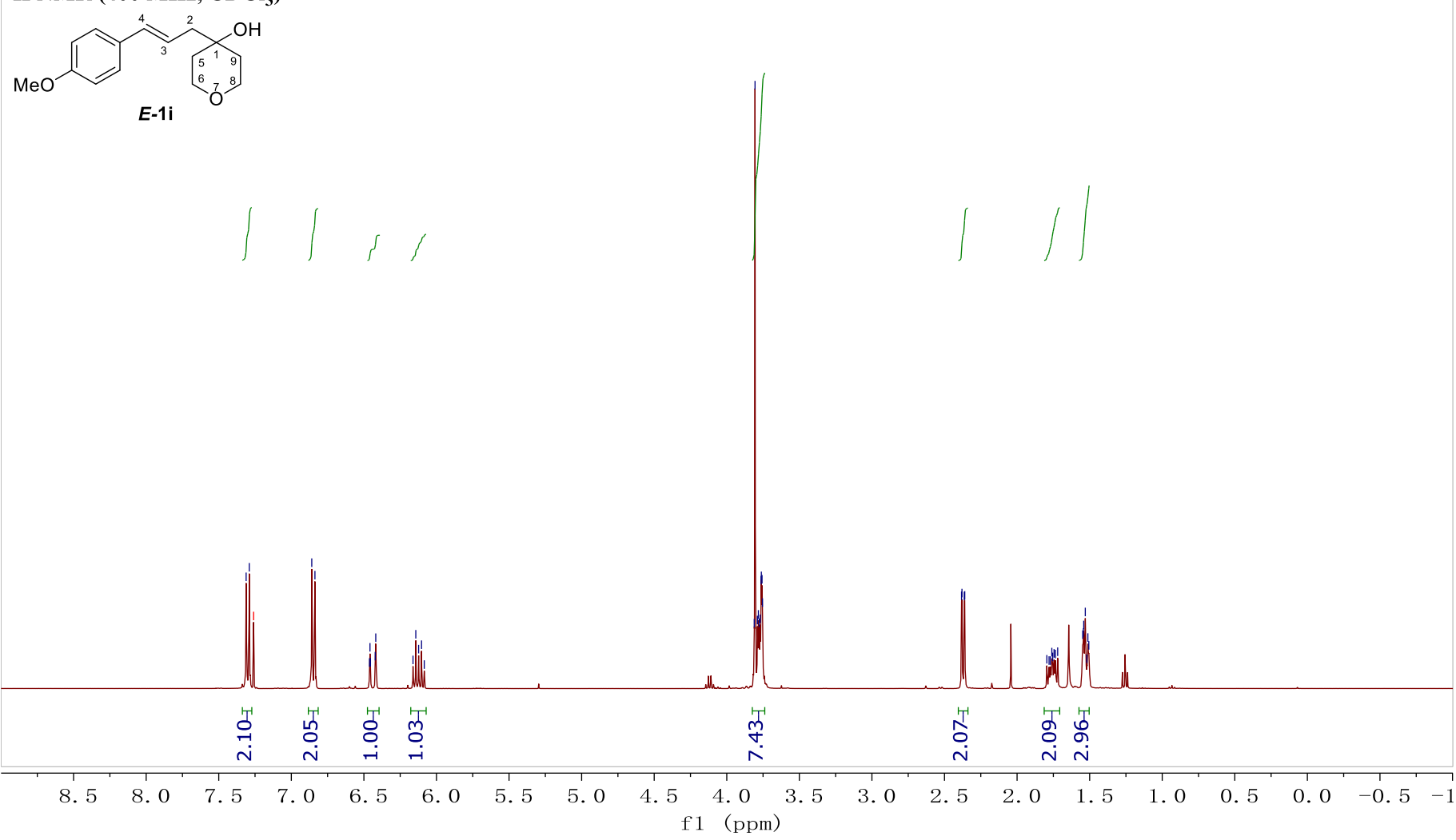

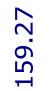

${ }^{13} \mathrm{C}$ NMR (100 MHz, $\left.\mathrm{CDCl}_{3}\right)$<smiles>CCCCCCC=Cc1ccc(OC)cc1</smiles>

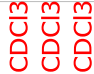

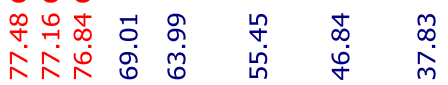

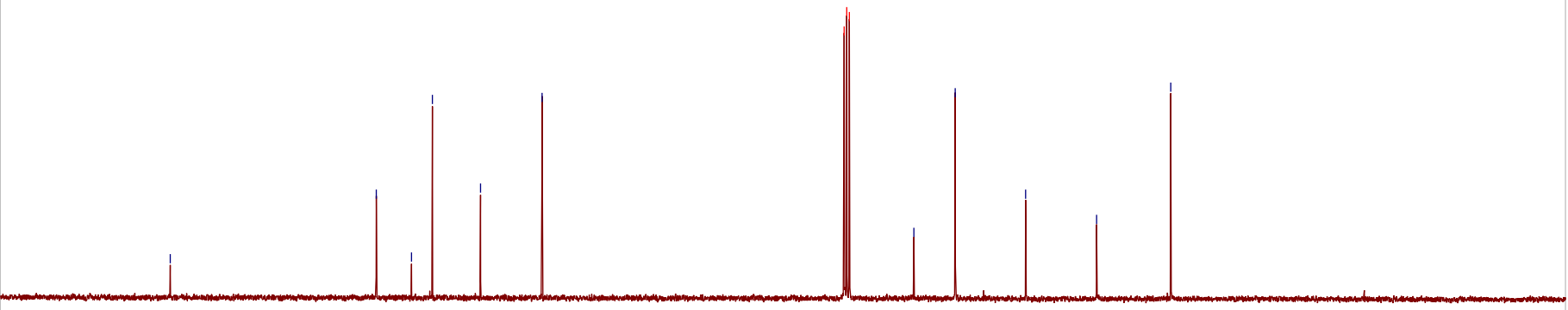


${ }^{1} \mathrm{H}$ NMR (400 MHz, $\left.\mathrm{CDCl}_{3}\right)$

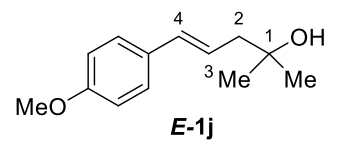

E-1j
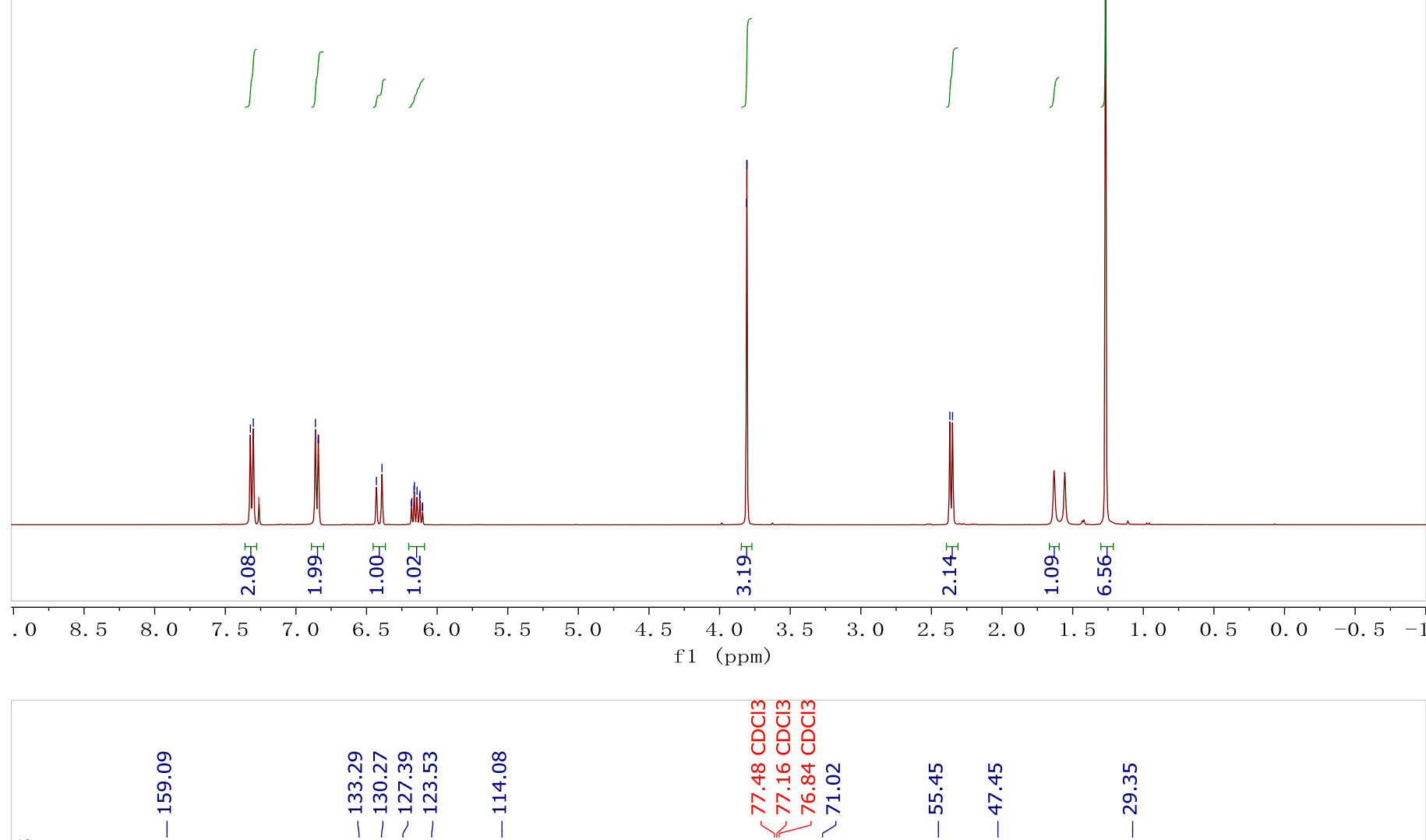

${ }^{13} \mathrm{C}$ NMR (100 MHz, $\left.\mathrm{CDCl}_{3}\right)$
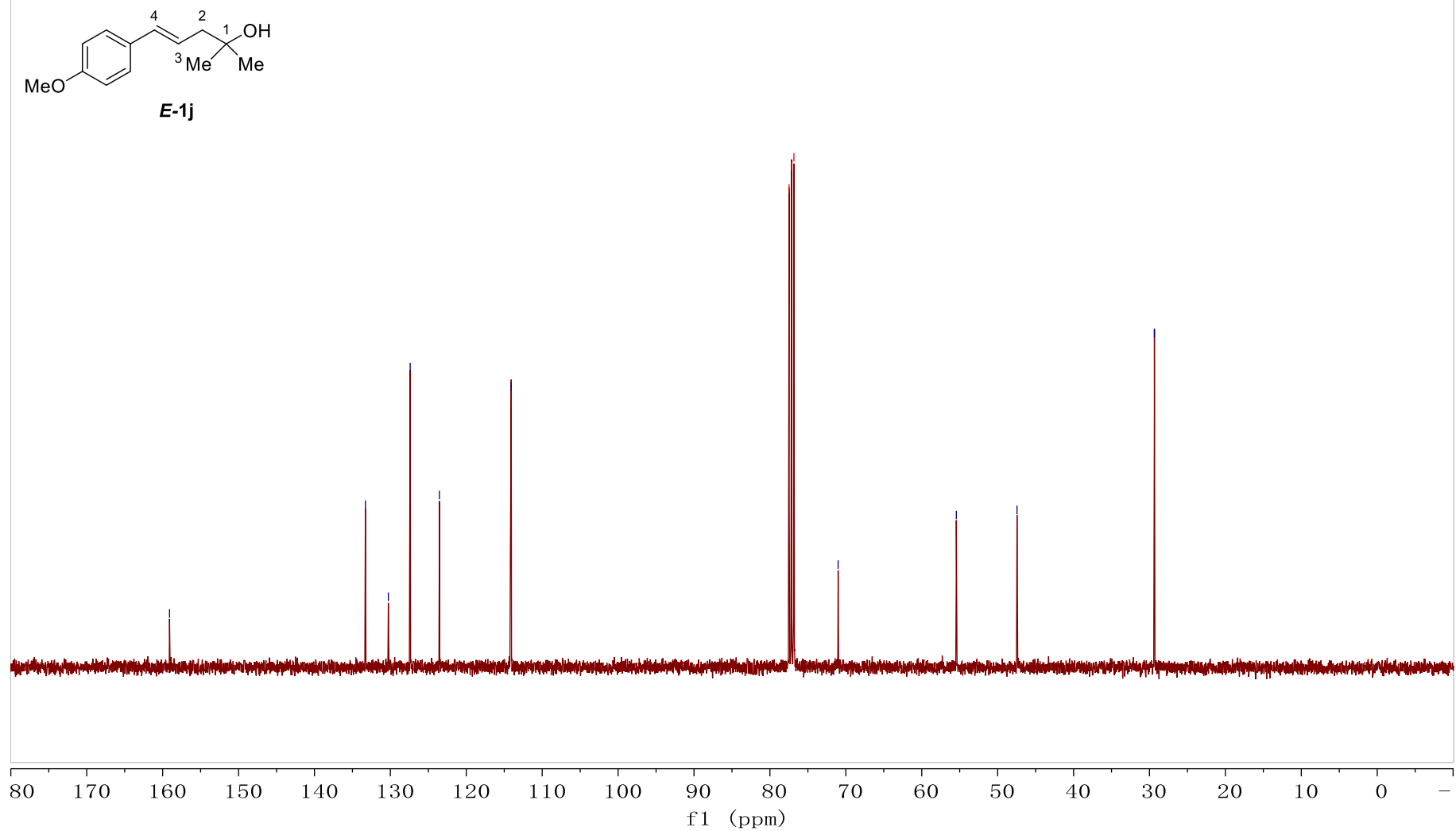
${ }^{1} \mathrm{H}$ NMR (500 MHz, $\mathrm{CDCl}_{3}$ )
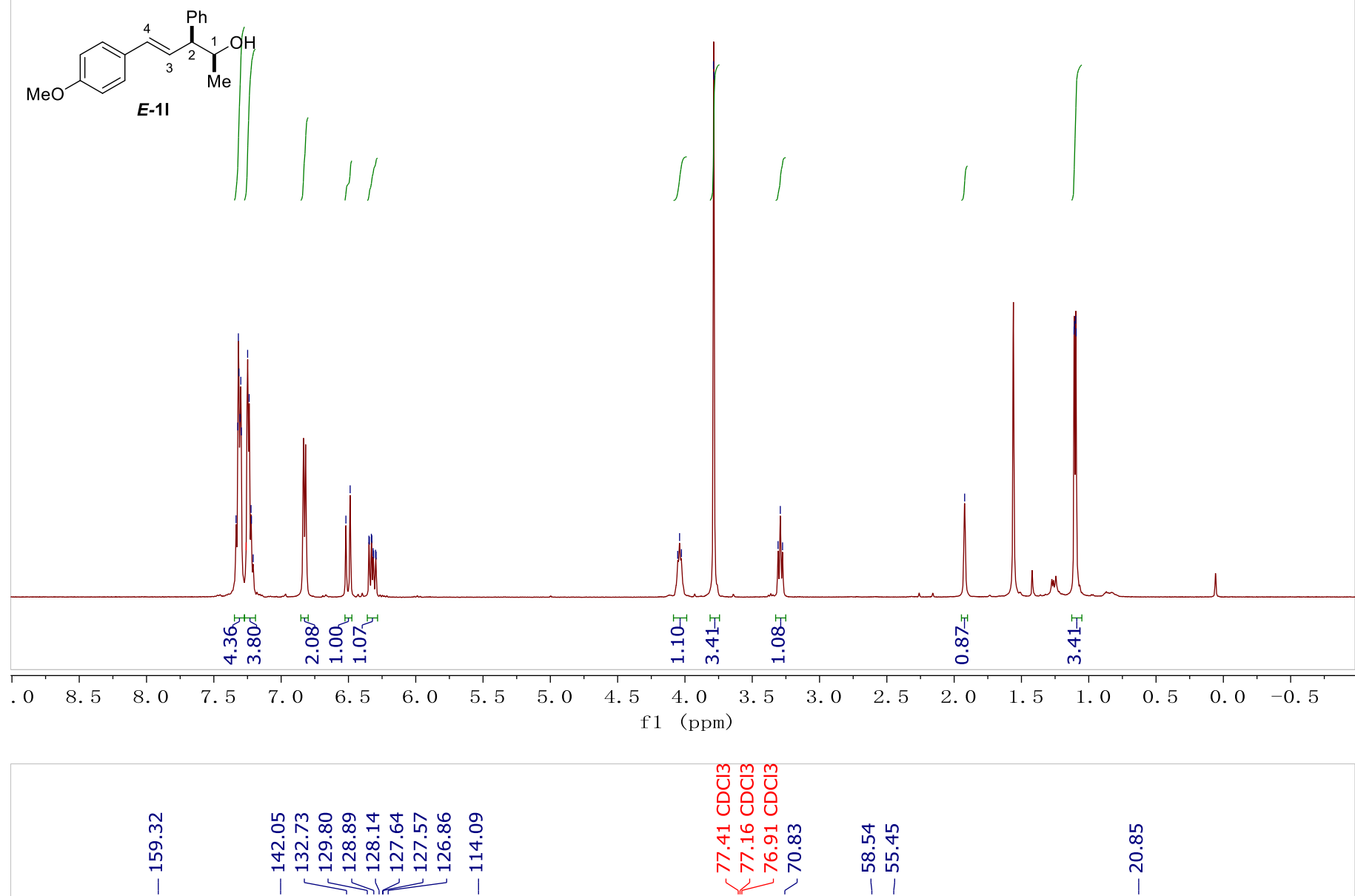

${ }^{13} \mathrm{C}$ NMR (125 MHz, $\left.\mathrm{CDCl}_{3}\right)$<smiles>COc1ccc(/C=C/C(c2ccccc2)C(O)O)cc1</smiles>

E-1I

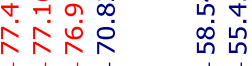
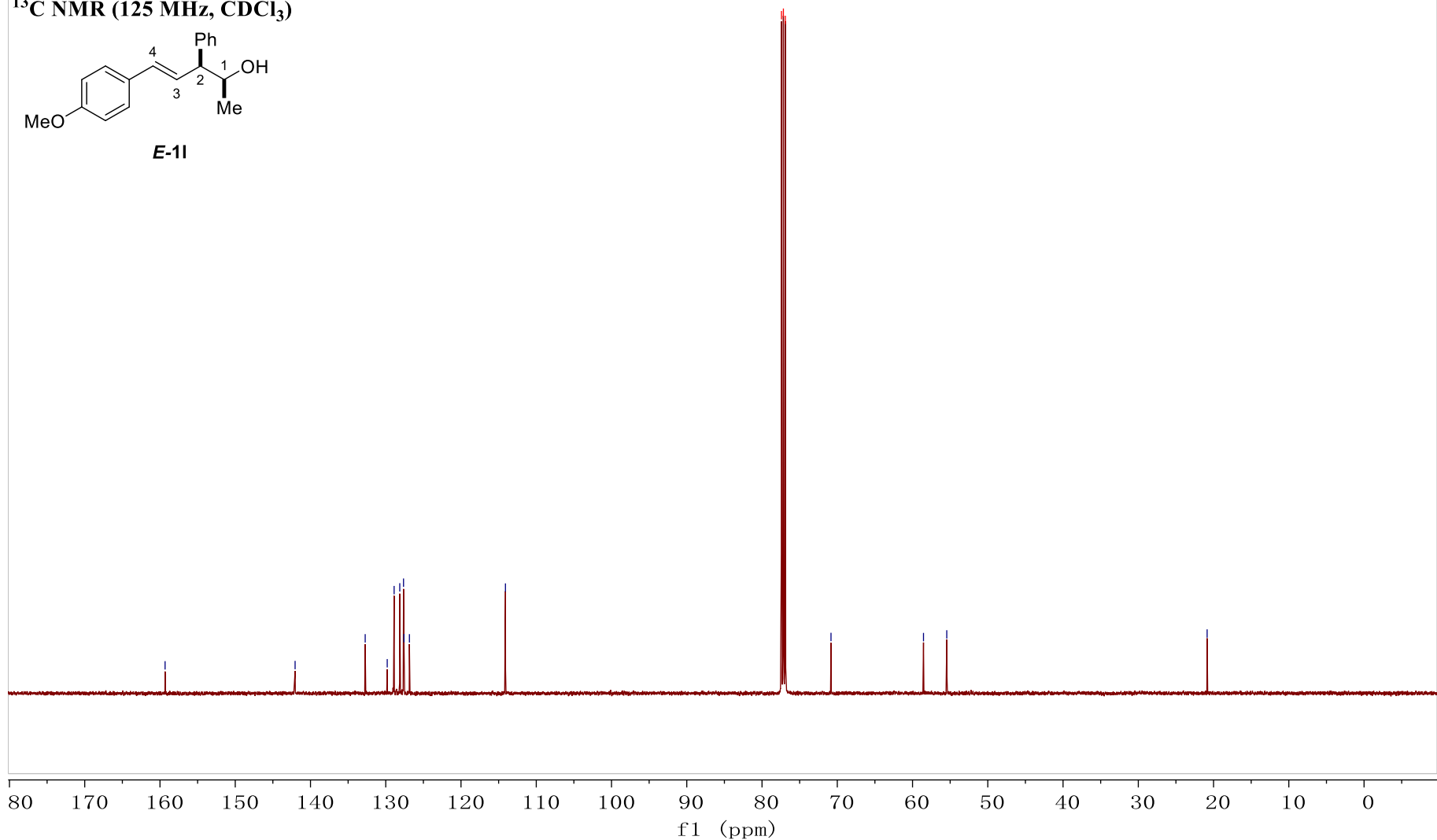


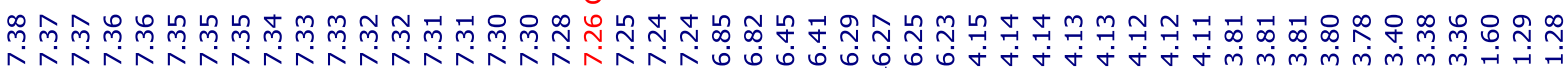

${ }^{1}$ H NMR (400 MHz, $\left.\mathrm{CDCl}_{3}\right)$
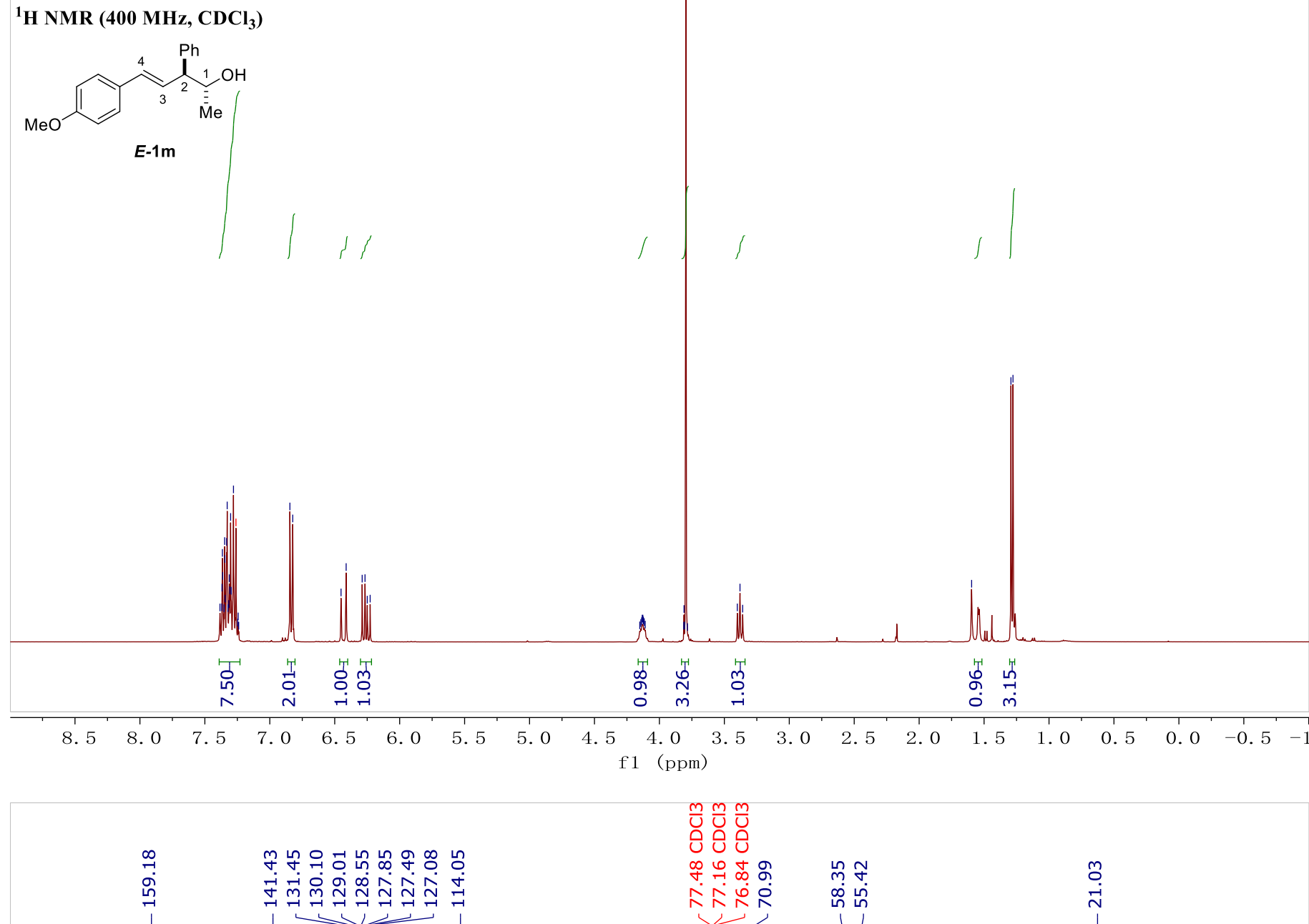

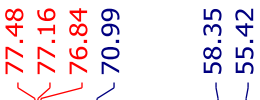

${ }^{13} \mathrm{C}$ NMR (100 MHz, $\mathrm{CDCl}_{3}$ )
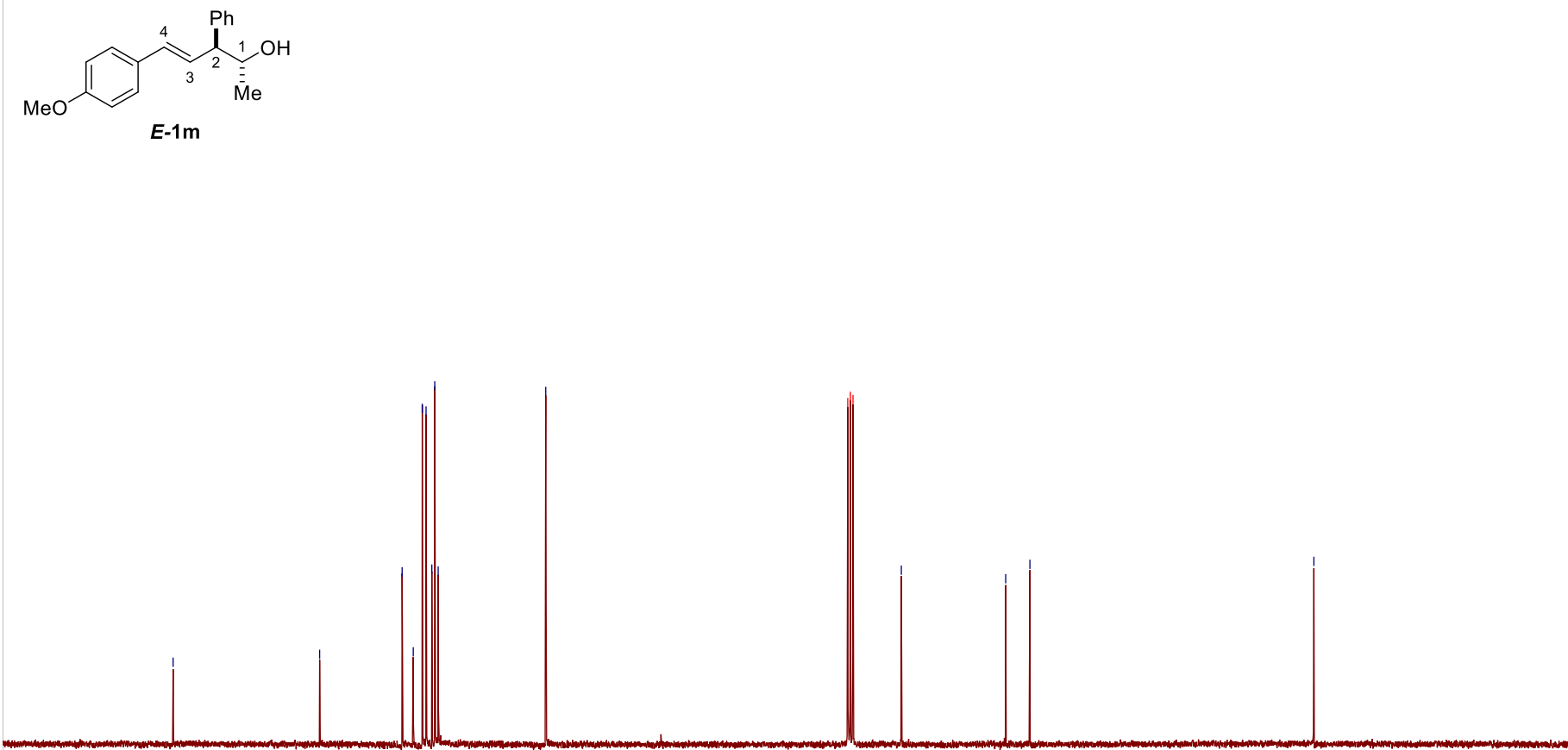

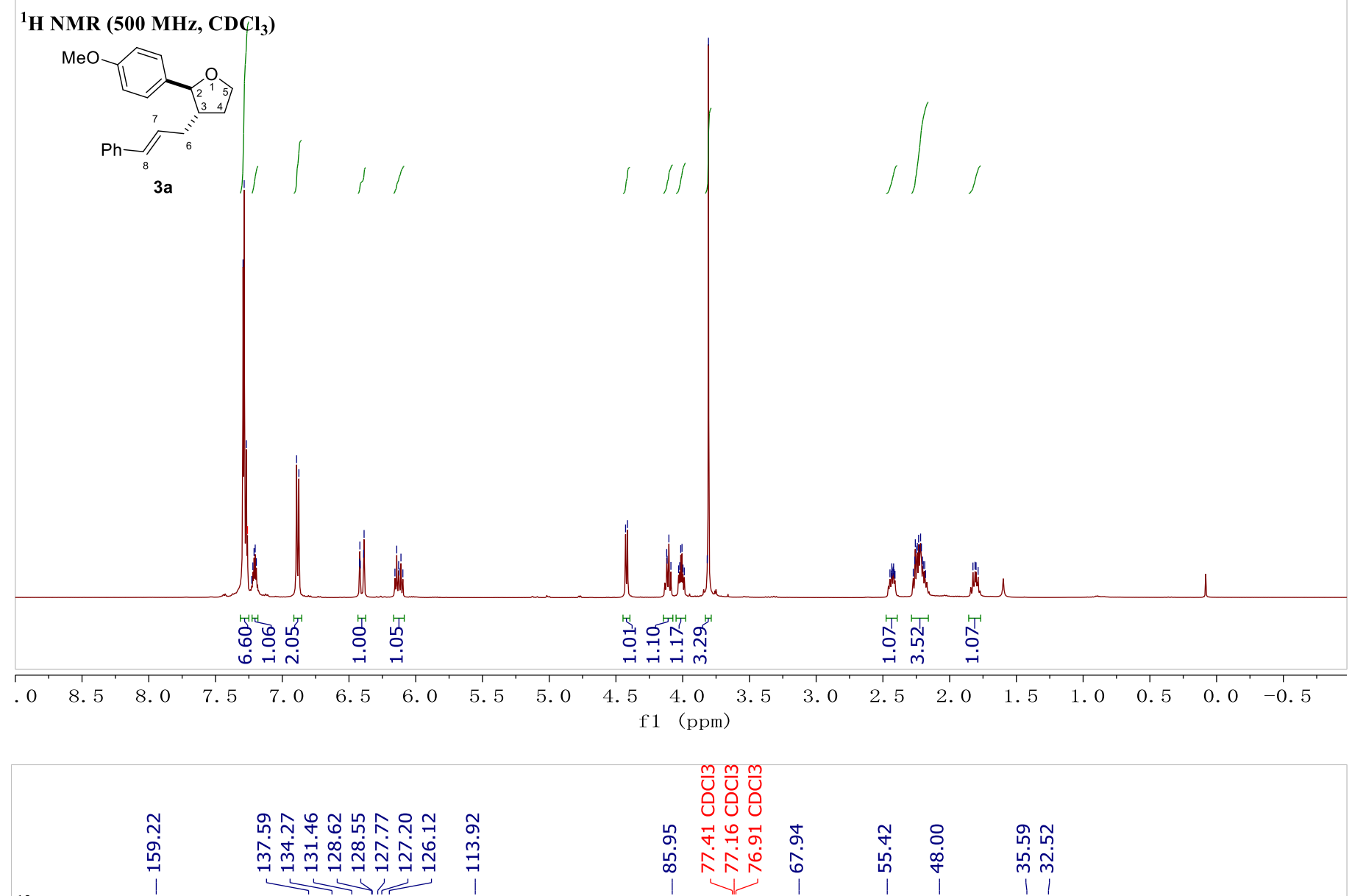

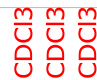

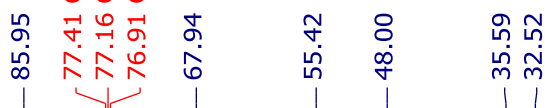

\section{${ }^{13} \mathrm{C}$ NMR (125 MHz, $\left.\mathrm{CDCl}_{3}\right)$}<smiles>CCCCC=CC=Cc1ccoc1-c1ccc(OC)cc1</smiles>

$3 a$

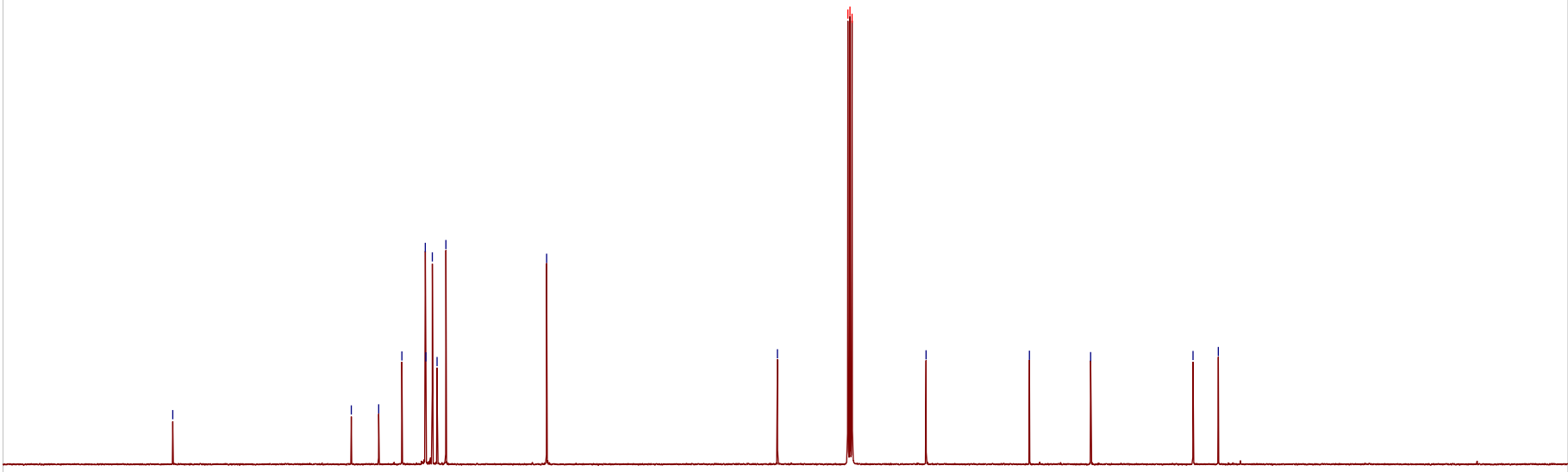




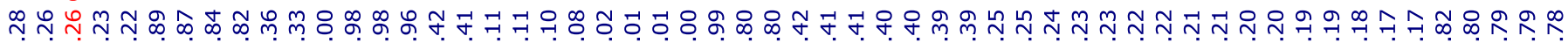

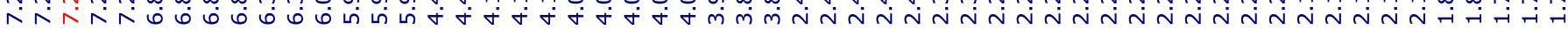

${ }^{1}$ H NMR (500 MHz, $\mathrm{CDCl}_{3}$ )
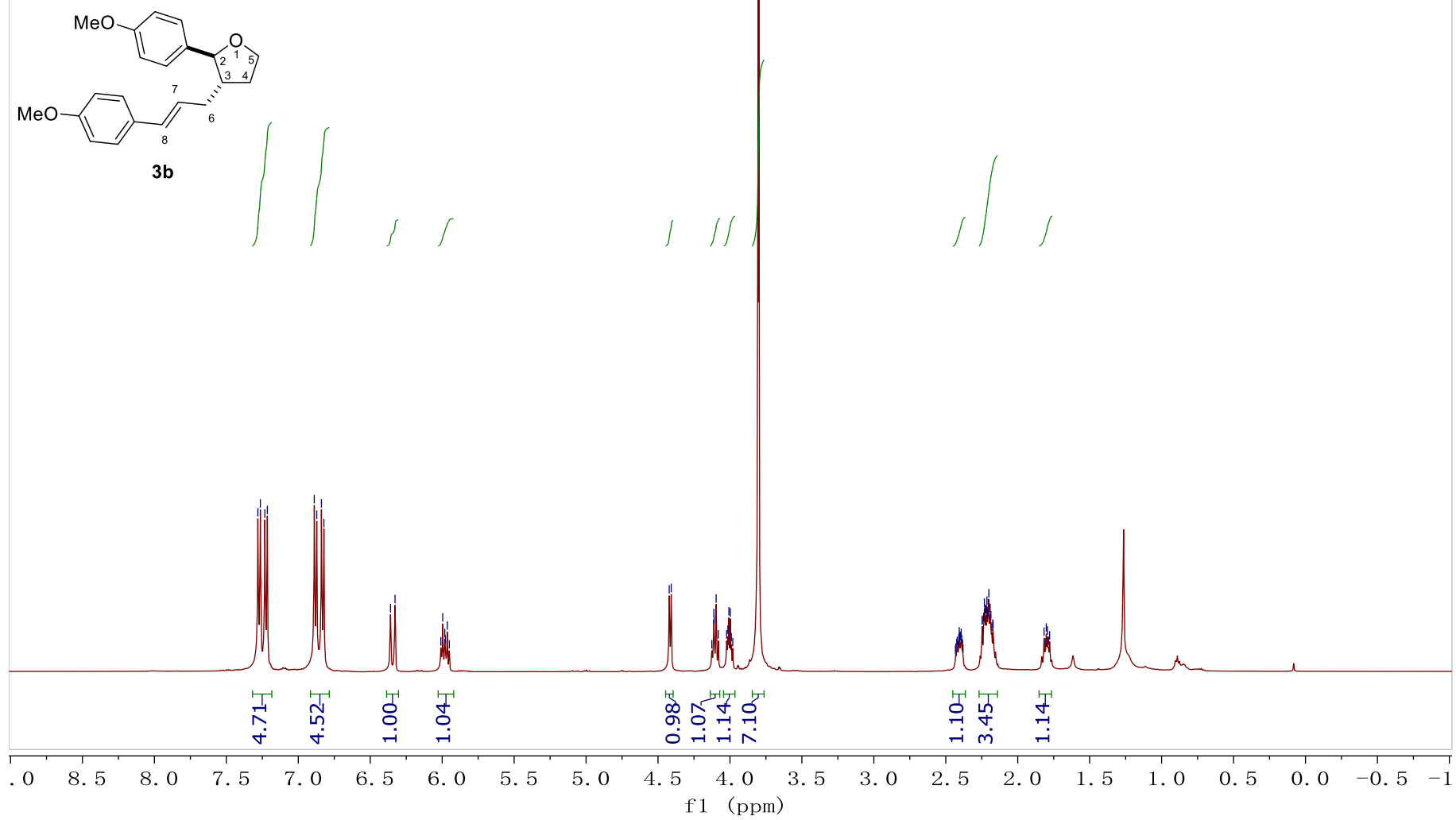

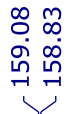

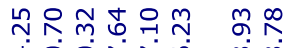

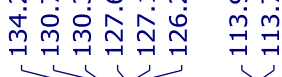

商商商

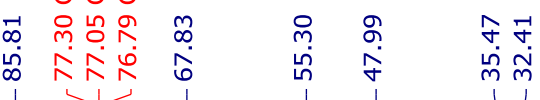

${ }^{13} \mathrm{C}$ NMR (125 MHz, $\left.\mathrm{CDCl}_{3}\right)$

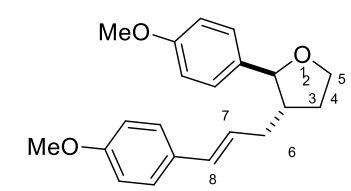

$3 b$
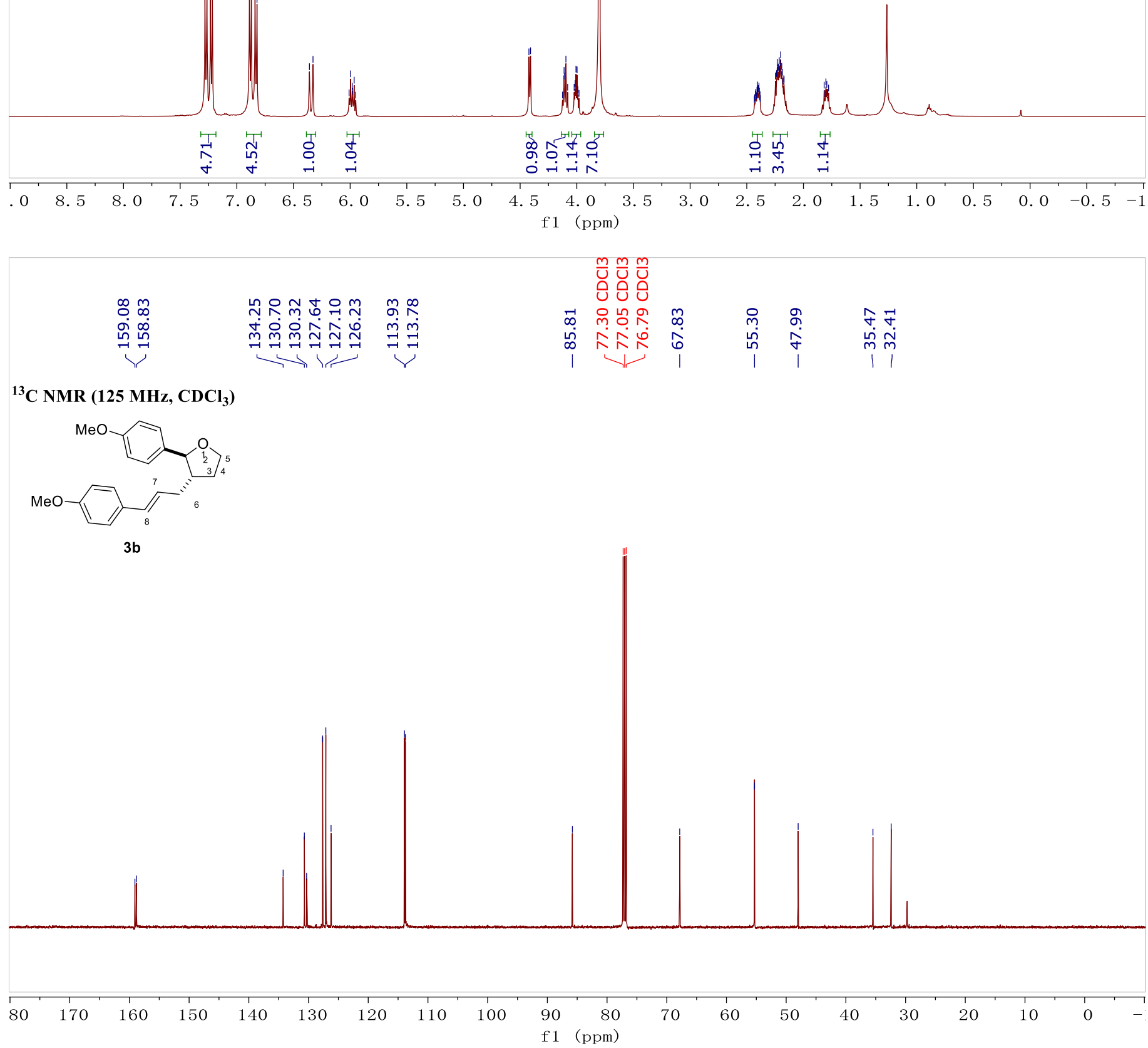

11 
${ }^{1} \mathrm{H}-{ }^{1} \mathrm{H}$ COSY - $90\left(500 \mathrm{MHz}, \mathrm{CDCl}_{3}\right)$

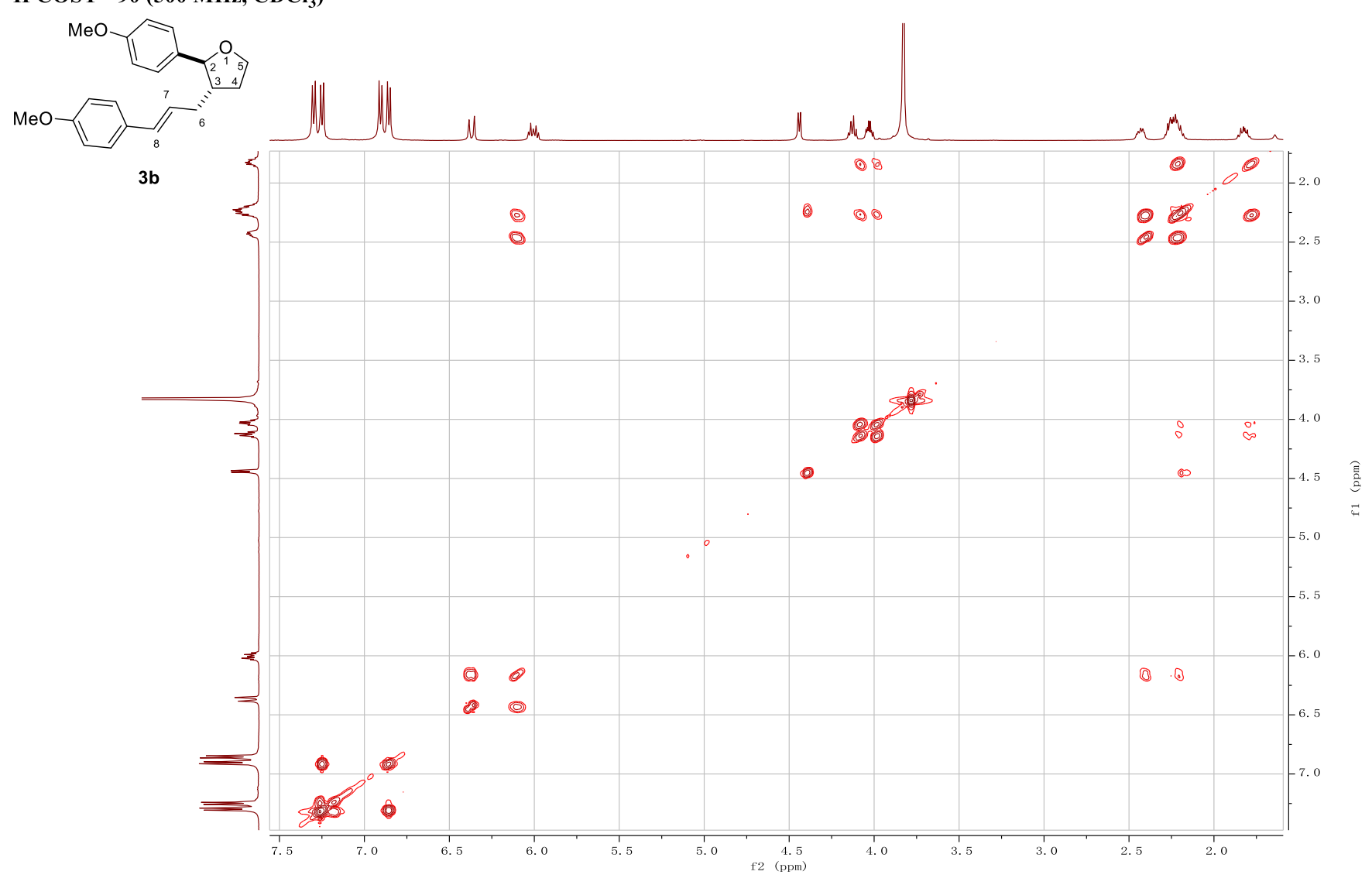

${ }^{1} \mathrm{H}(500 \mathrm{MHz})-{ }^{13} \mathrm{C}(125 \mathrm{MHz}) \mathrm{HSQC}\left(\mathrm{CDCl}_{3}\right)$

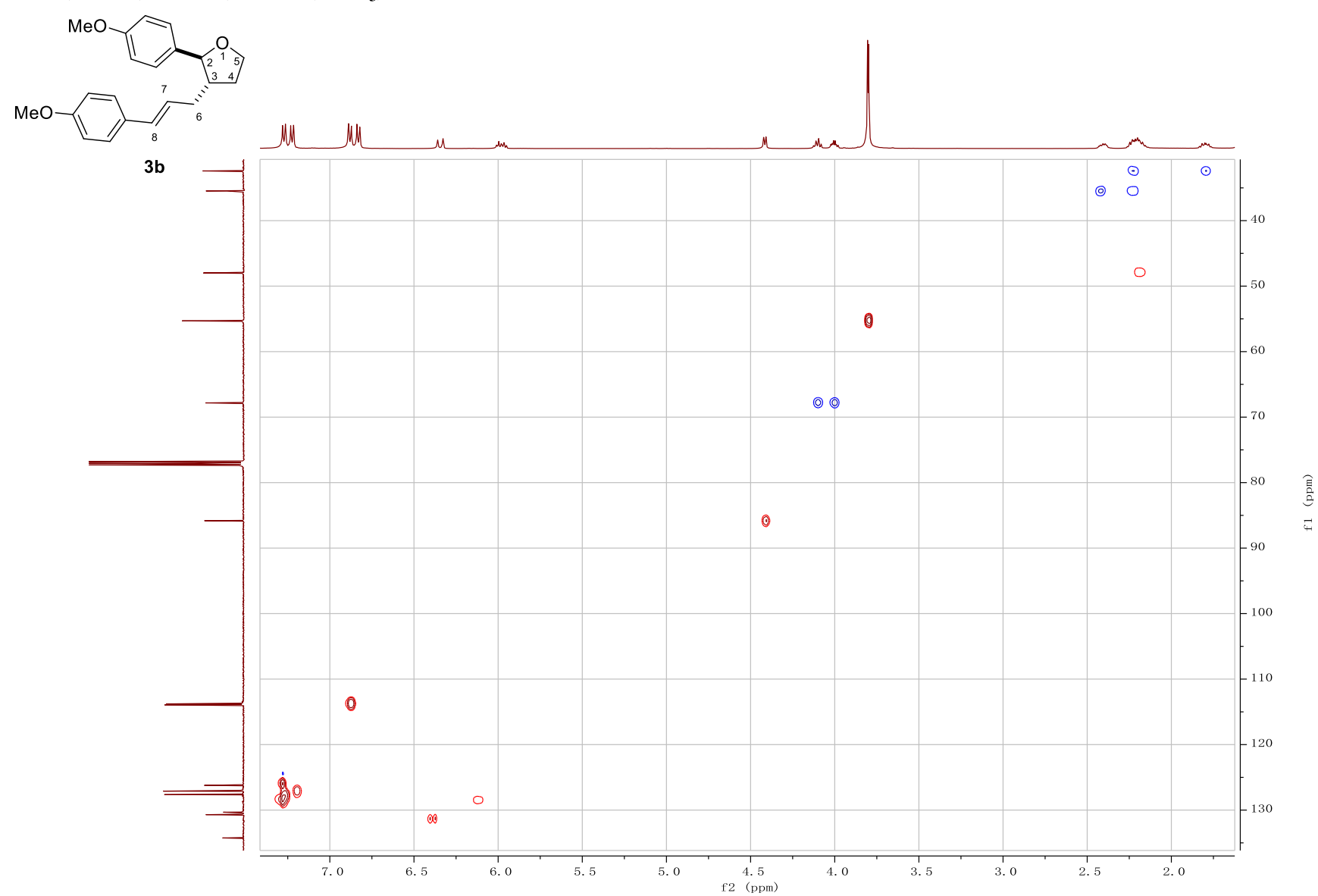




$$
\begin{aligned}
& 7 \\
& =1 \\
& =11
\end{aligned}
$$


บั

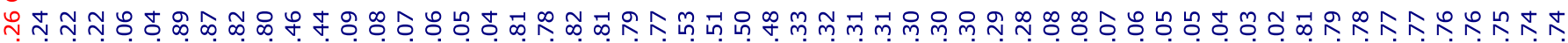

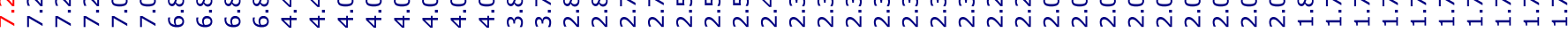

\section{${ }^{1} \mathrm{H}$ NMR (400 MHz, $\left.\mathrm{CDCl}_{3}\right)$}

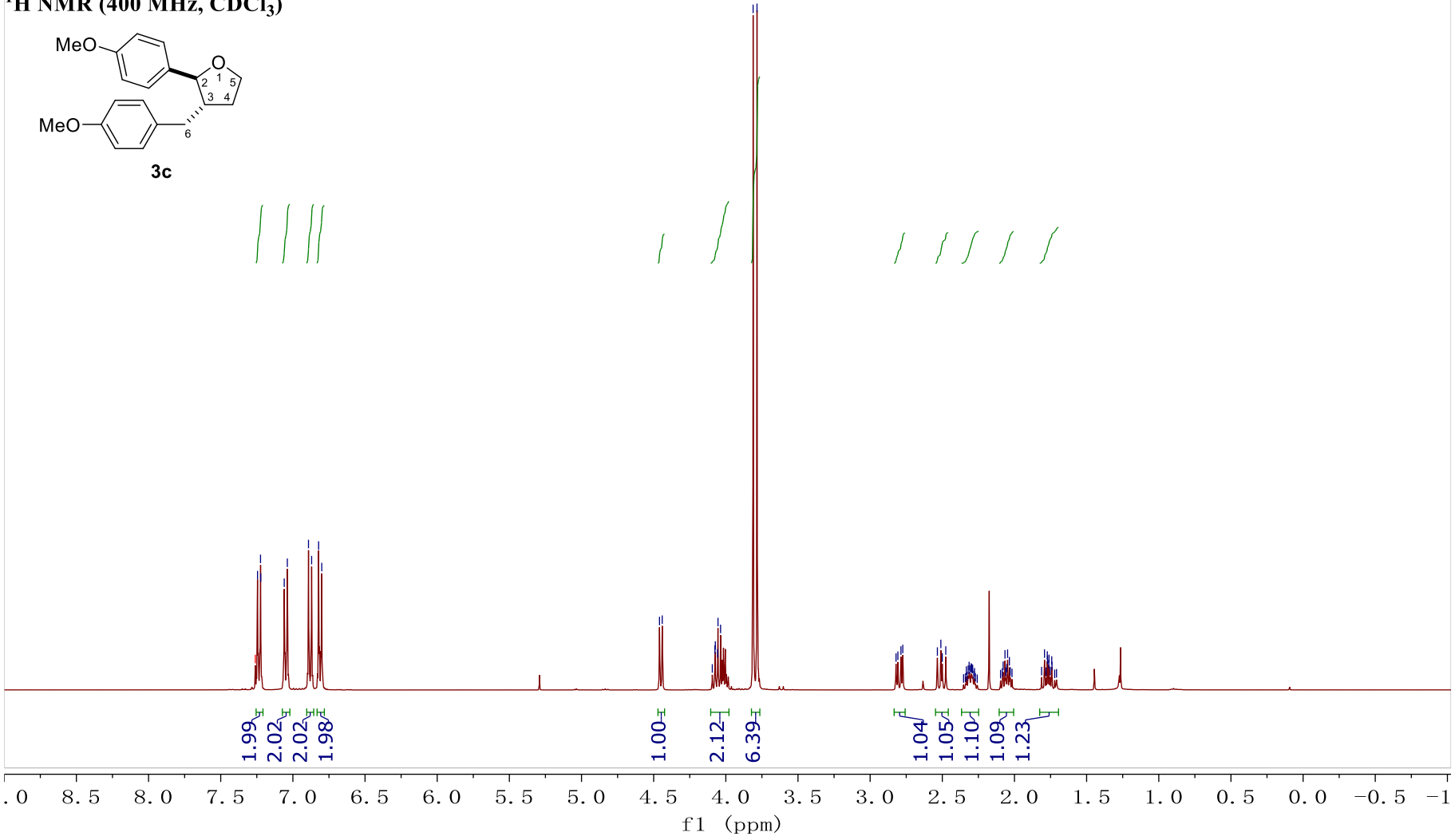

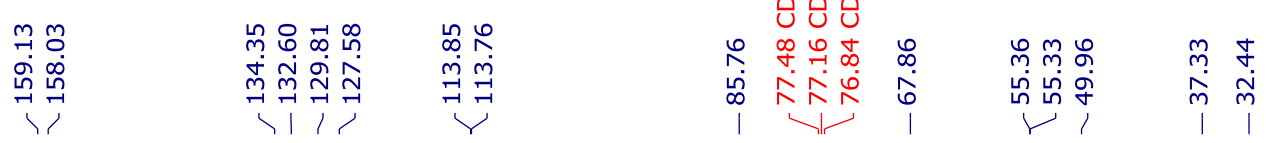

${ }^{13} \mathrm{C}$ NMR (100 MHz, $\left.\mathrm{CDCl}_{3}\right)$
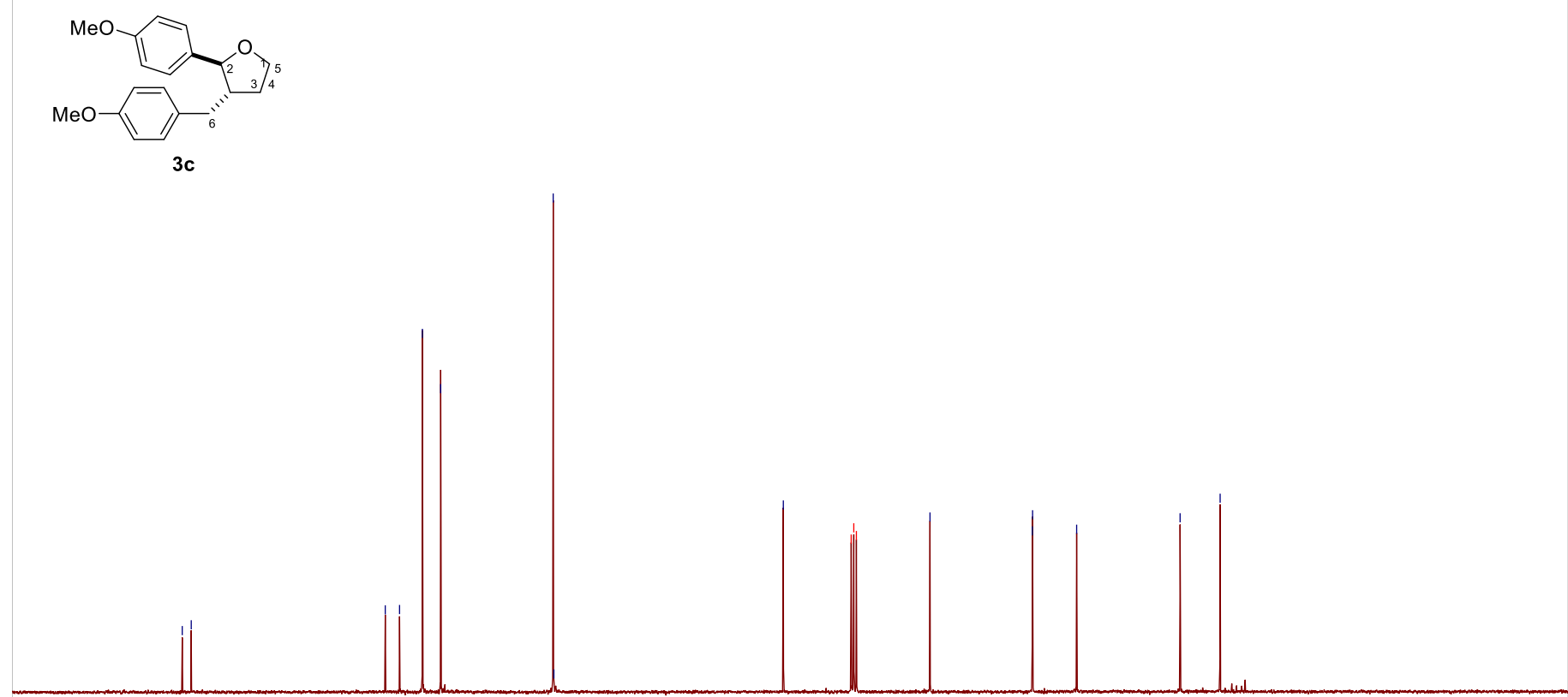

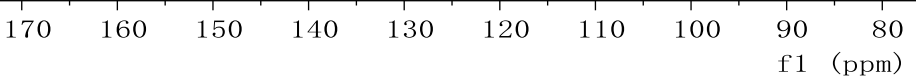


${ }^{1} \mathrm{H}-{ }^{1} \mathrm{H} \operatorname{COSY}$ - $90\left(400 \mathrm{MHz}, \mathrm{CDCl}_{3}\right)$

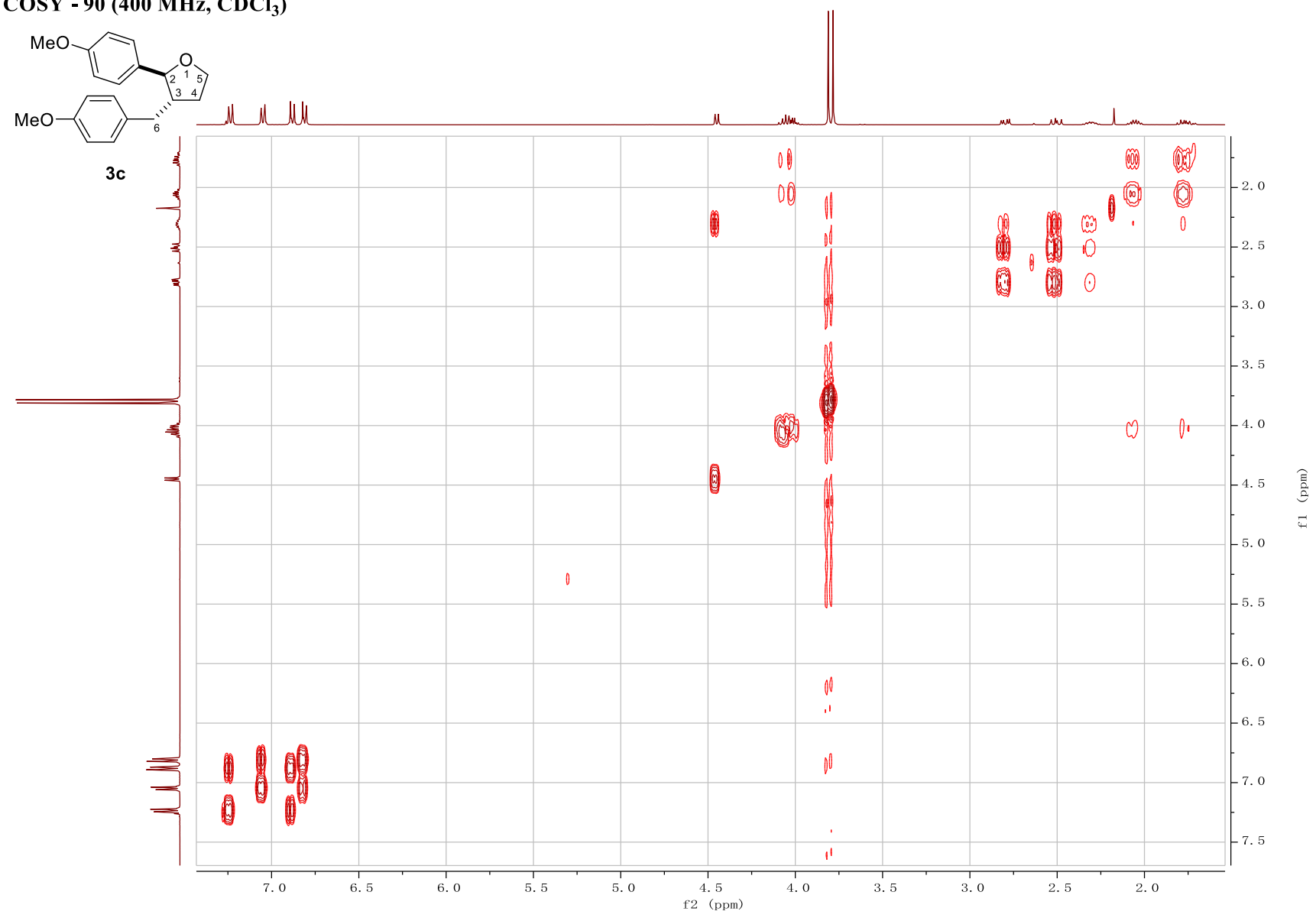

${ }^{1} \mathrm{H}(400 \mathrm{MHz})-{ }^{13} \mathrm{C}(100 \mathrm{MHz}) \mathrm{HSQC}\left(\mathrm{CDCl}_{3}\right)$
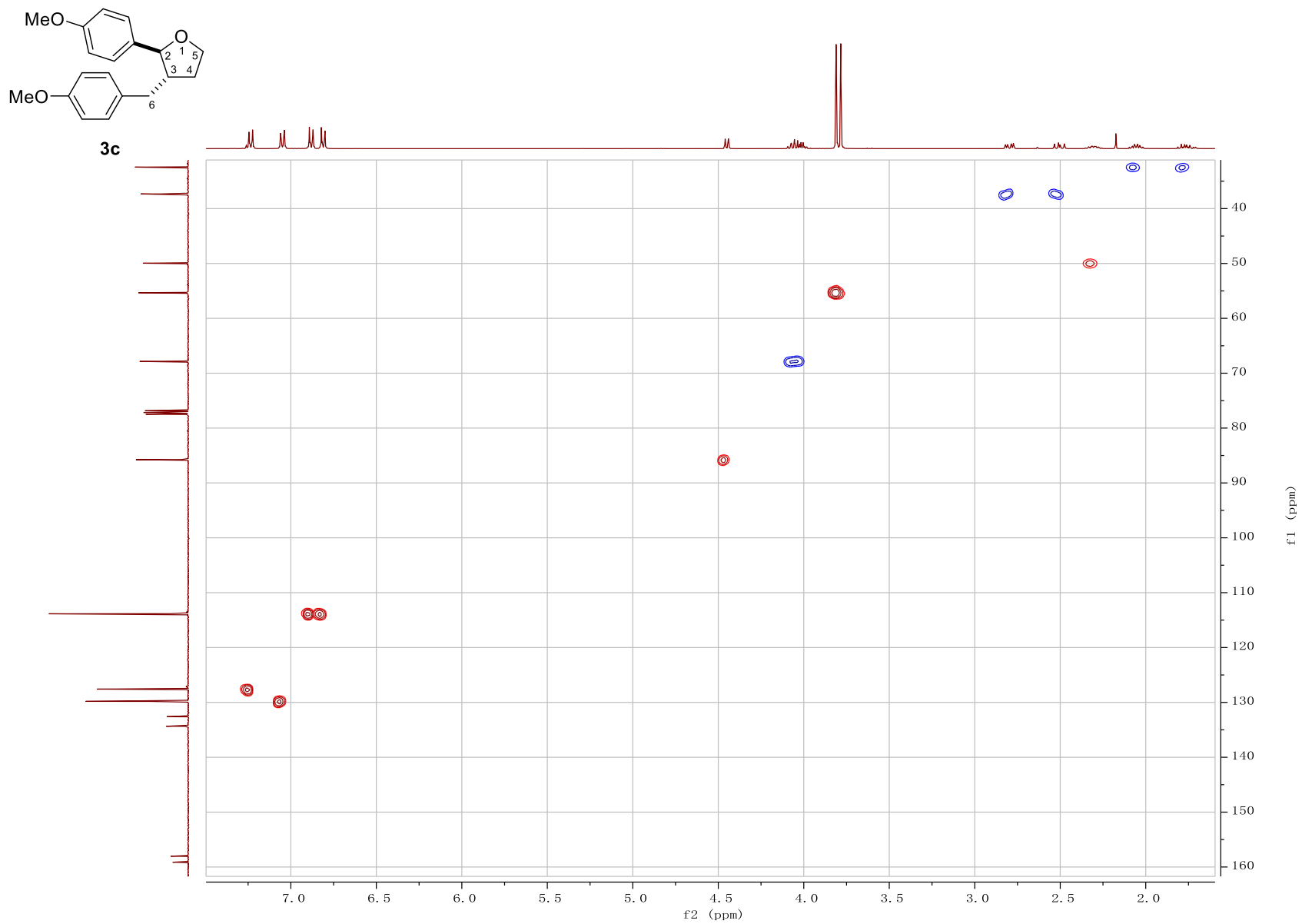


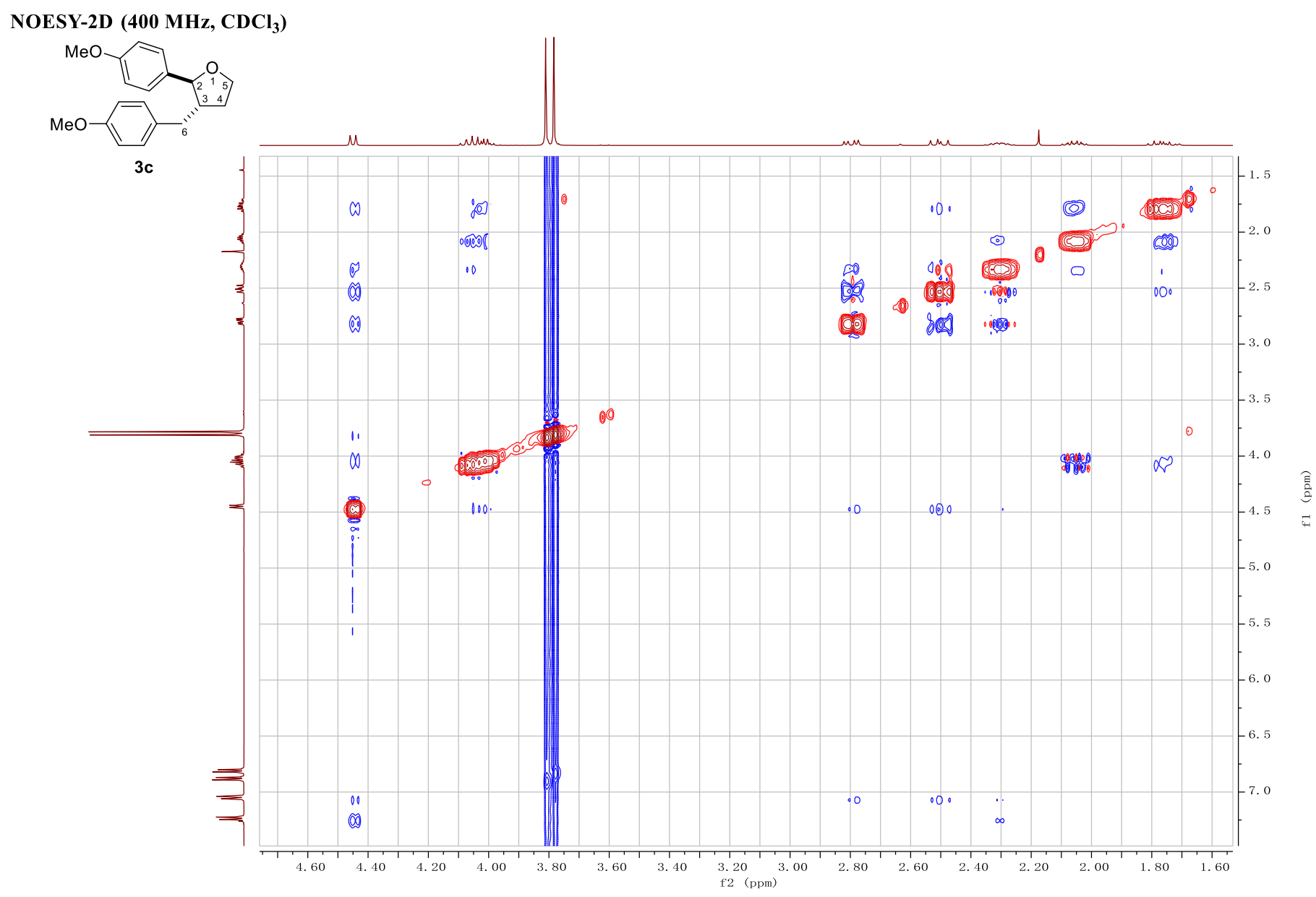

S21 
${ }^{1} \mathrm{H}$ NMR (400 MHz, $\left.\mathrm{CDCl}_{3}\right)$
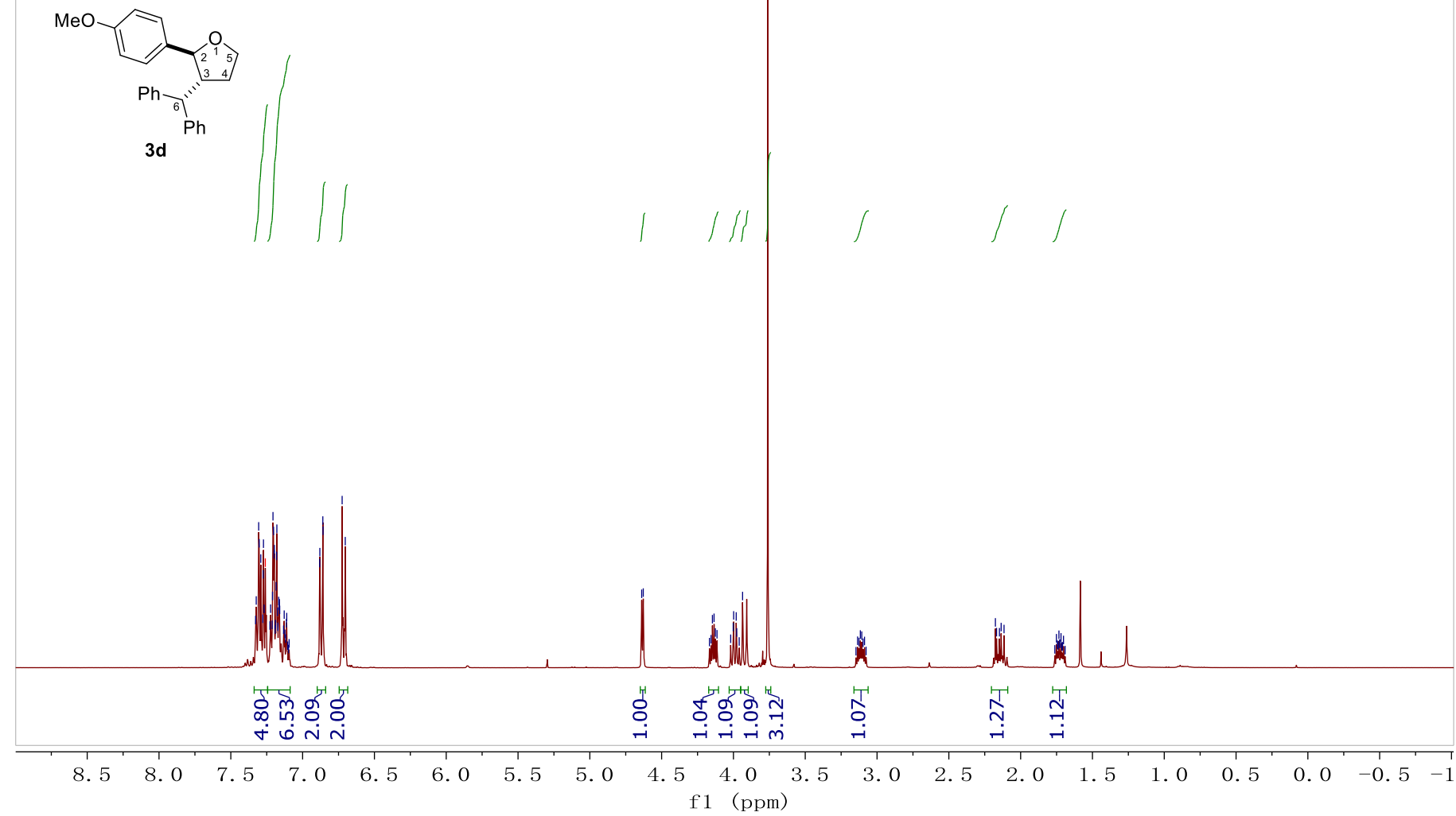

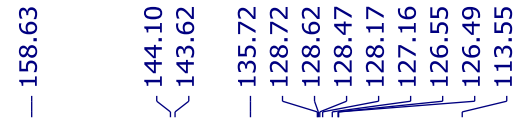

产商商

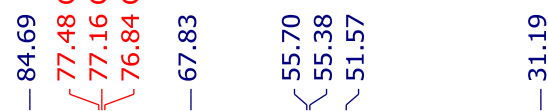

${ }^{13} \mathrm{C}$ NMR (100 MHz, $\left.\mathrm{CDCl}_{3}\right)$<smiles>COc1ccc(-c2occc2-c2ccccc2)cc1</smiles>

3d

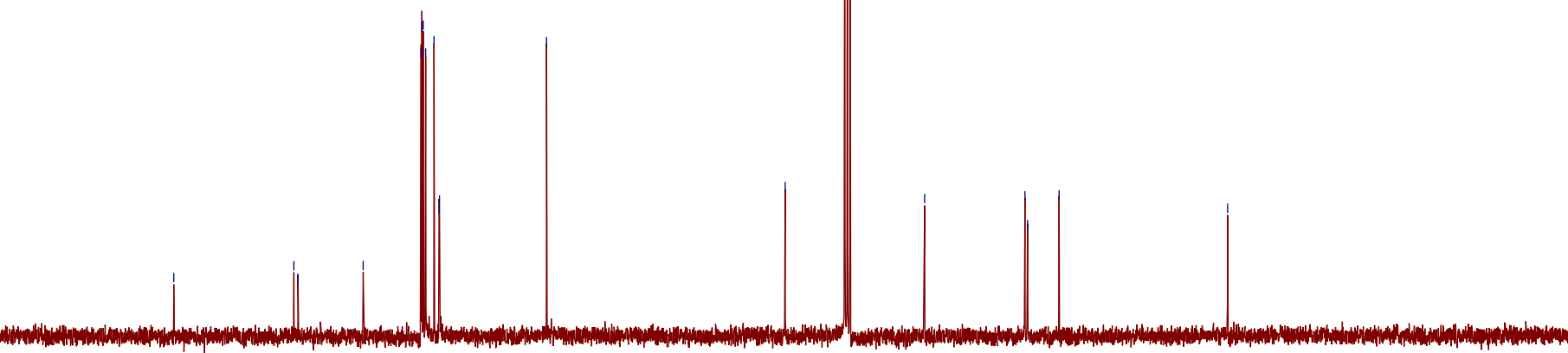

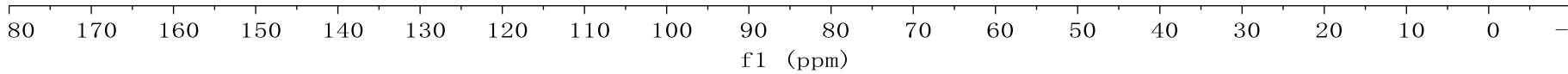




\section{${ }^{1} \mathrm{H}$ NMR (500 MHz, $\mathrm{CDCl}_{3}$ )}

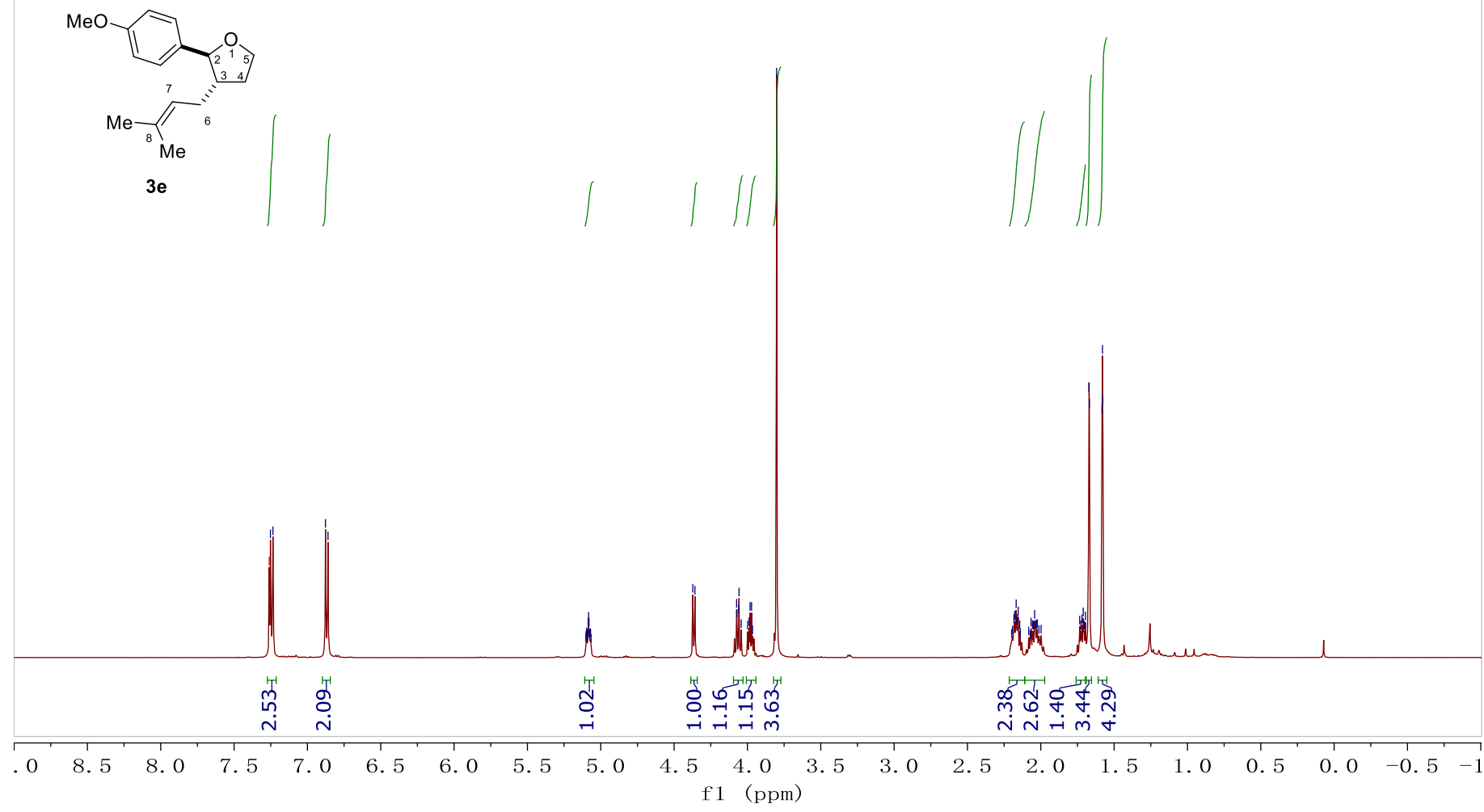

\begin{tabular}{|c|c|c|c|c|c|c|c|c|}
\hline 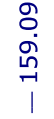 & 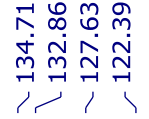 & $\begin{array}{l}-1 \\
\infty \\
\stackrel{9}{\rightrightarrows} \\
-1 \\
1\end{array}$ & $\begin{array}{l}m \\
\infty \\
1 \\
\infty \\
1\end{array}$ & 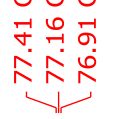 & 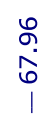 & 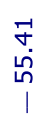 & $\begin{array}{c}\hat{m} \\
\infty \\
\infty \\
\dot{\sigma} \\
1\end{array}$ & 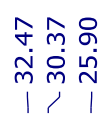 \\
\hline
\end{tabular}

${ }^{13} \mathrm{C}$ NMR (125 MHz, $\left.\mathrm{CDCl}_{3}\right)$

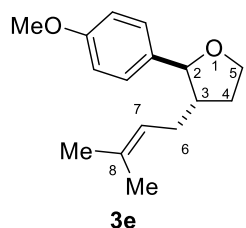


$\frac{m}{\mathrm{U}}$

뉴슈슈

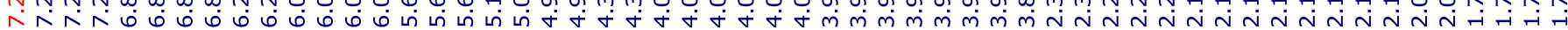

${ }^{1} \mathrm{H}$ NMR (500 MHz, $\left.\mathrm{CDCl}_{3}\right)$

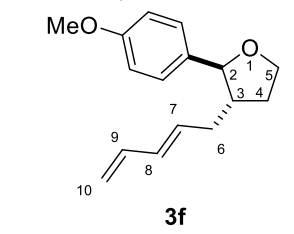

$3 f$
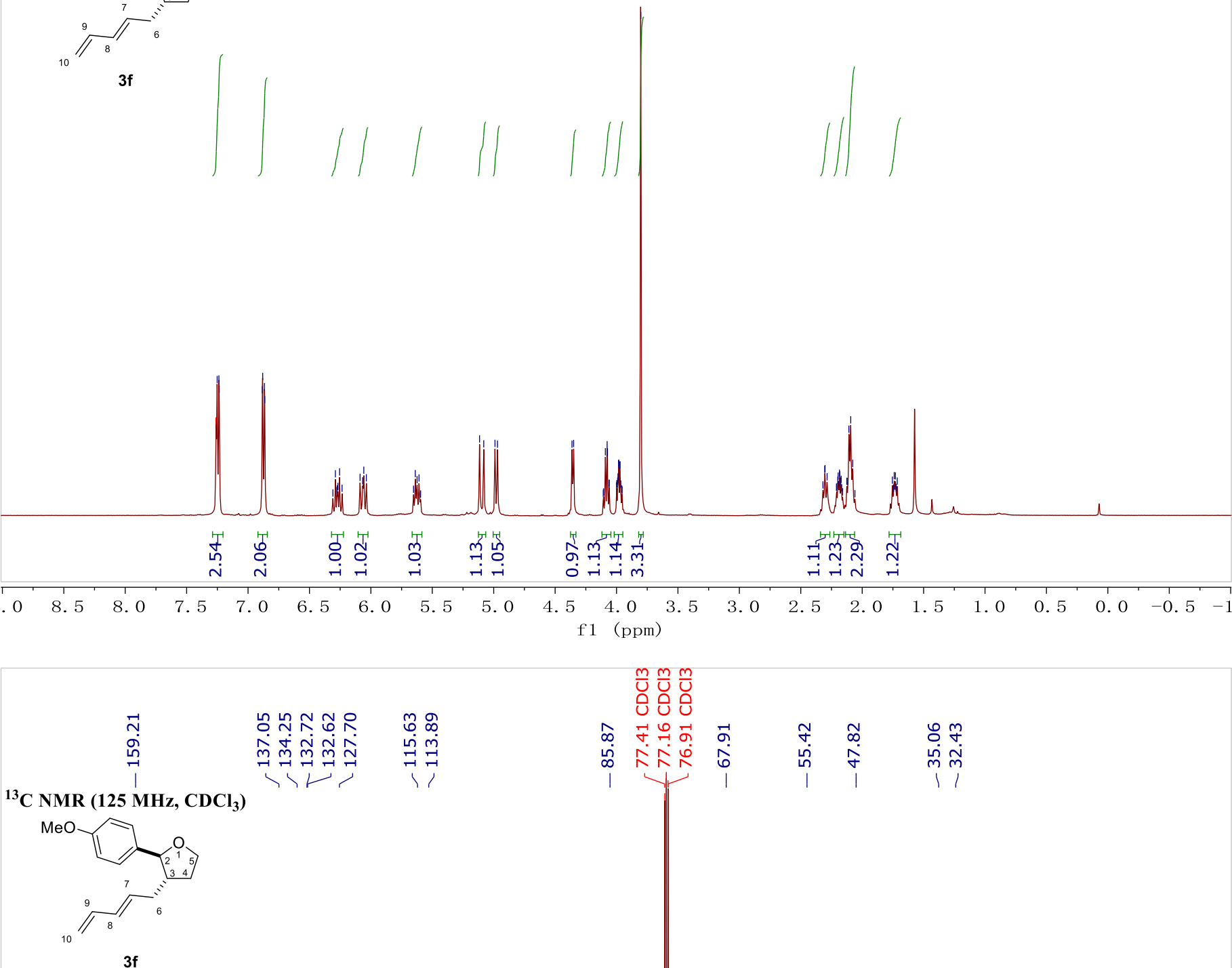

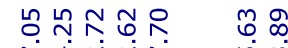

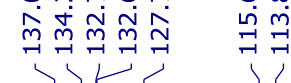

商旁融

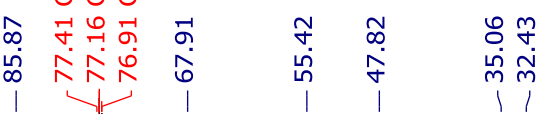

${ }^{13} \mathrm{C}$ NMR (125 MHz, $\left.\mathrm{CDCl}_{3}\right)$
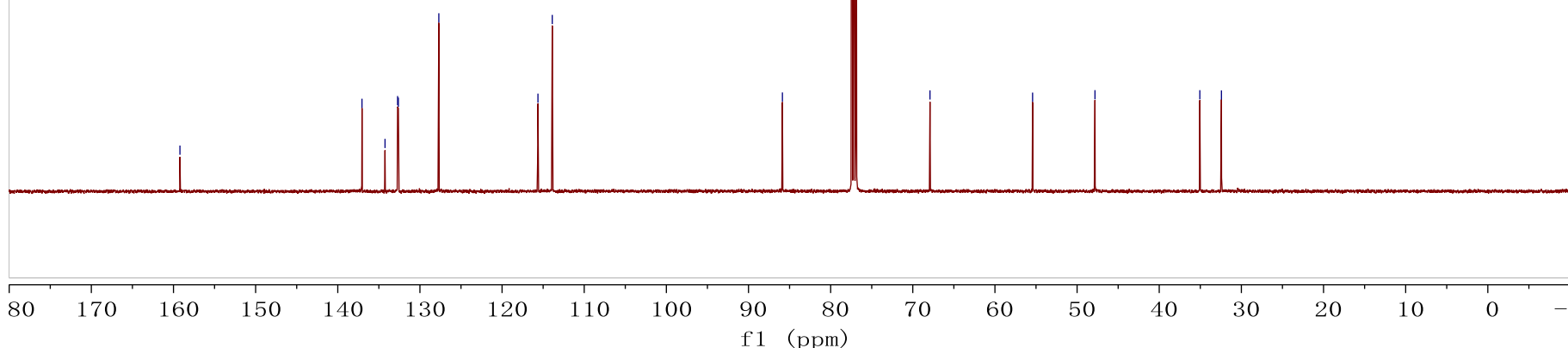


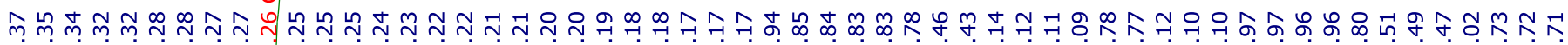

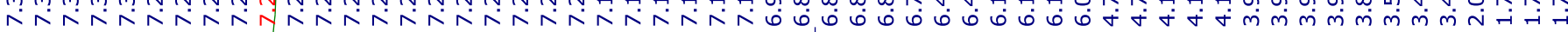

${ }^{1} \mathrm{H}$ NMR (500 MHz, $\left.\mathrm{CDCl}_{3}\right)$

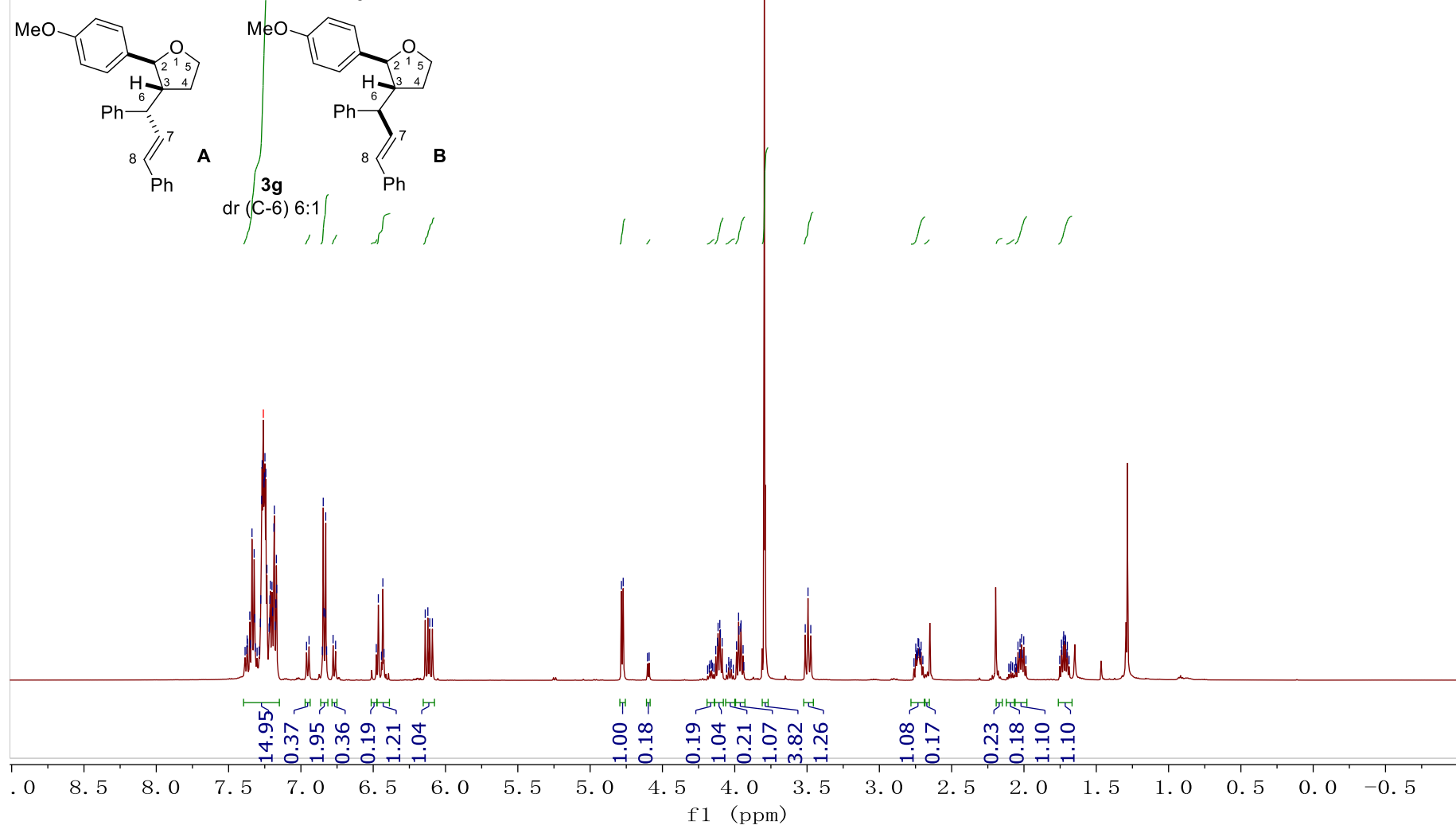

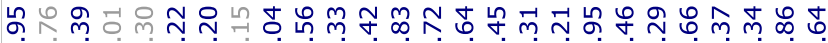

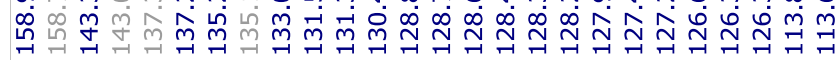

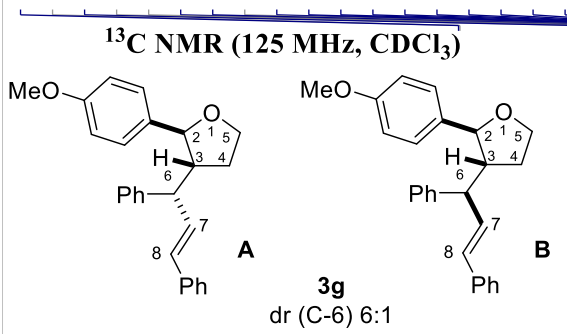

\section{产 $\frac{m}{\bar{U}}$}

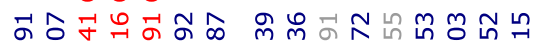

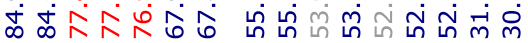

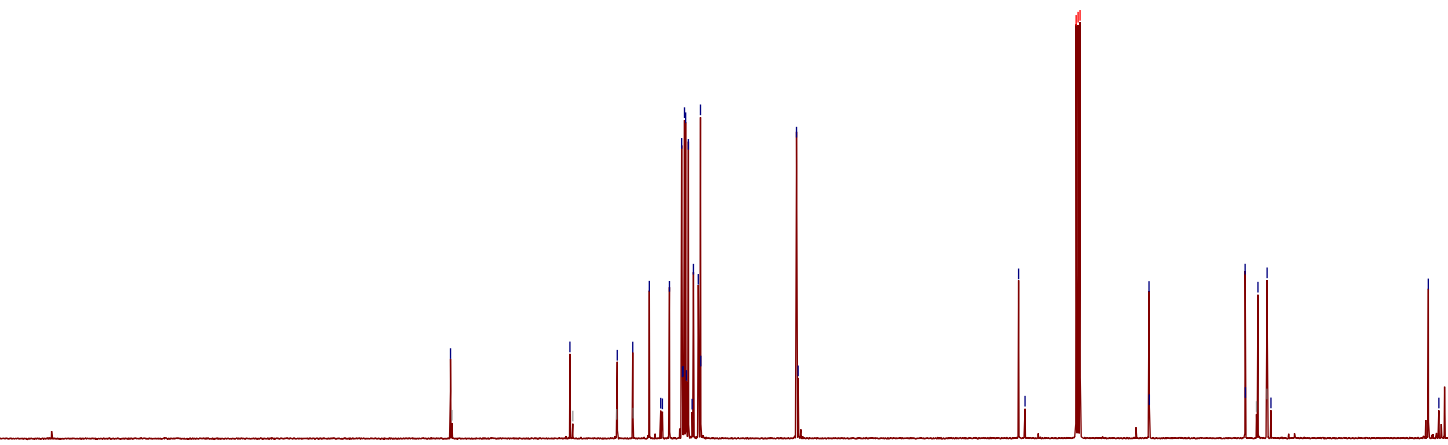

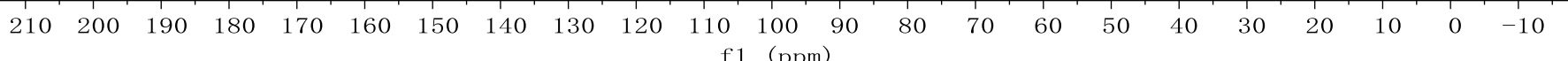


$\frac{m}{\mathrm{U}}$

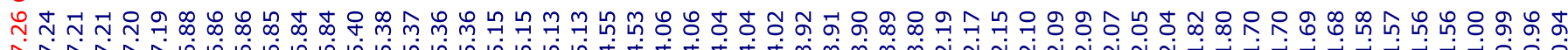

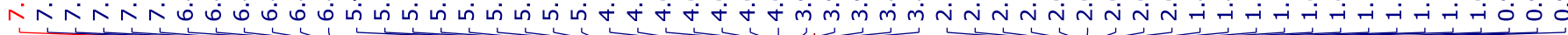

${ }^{1} \mathrm{H}$ NMR (400 MHz, $\left.\mathrm{CDCl}_{3}\right)$

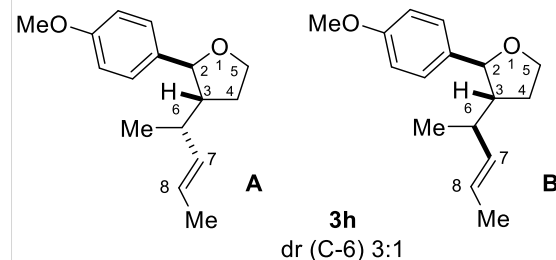

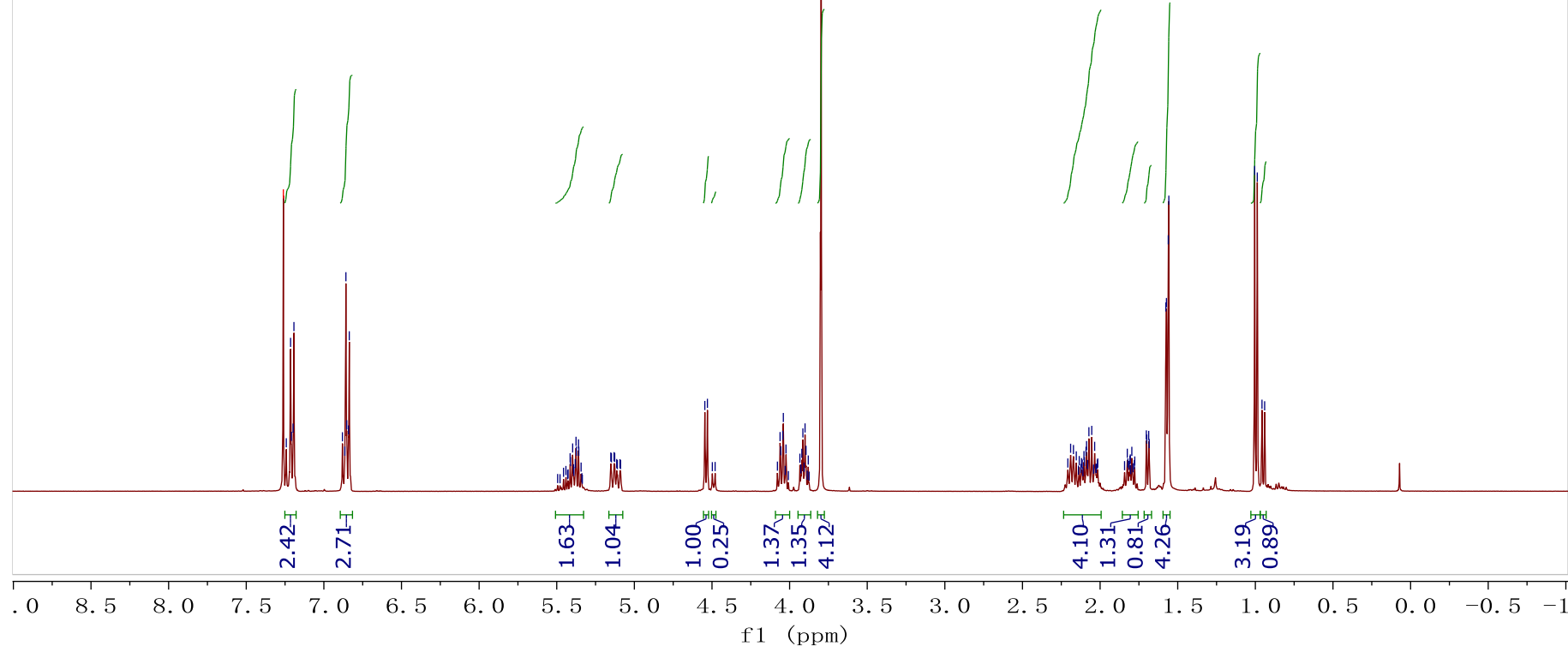

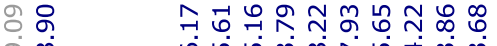

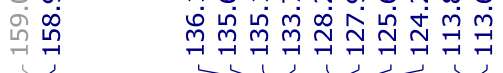
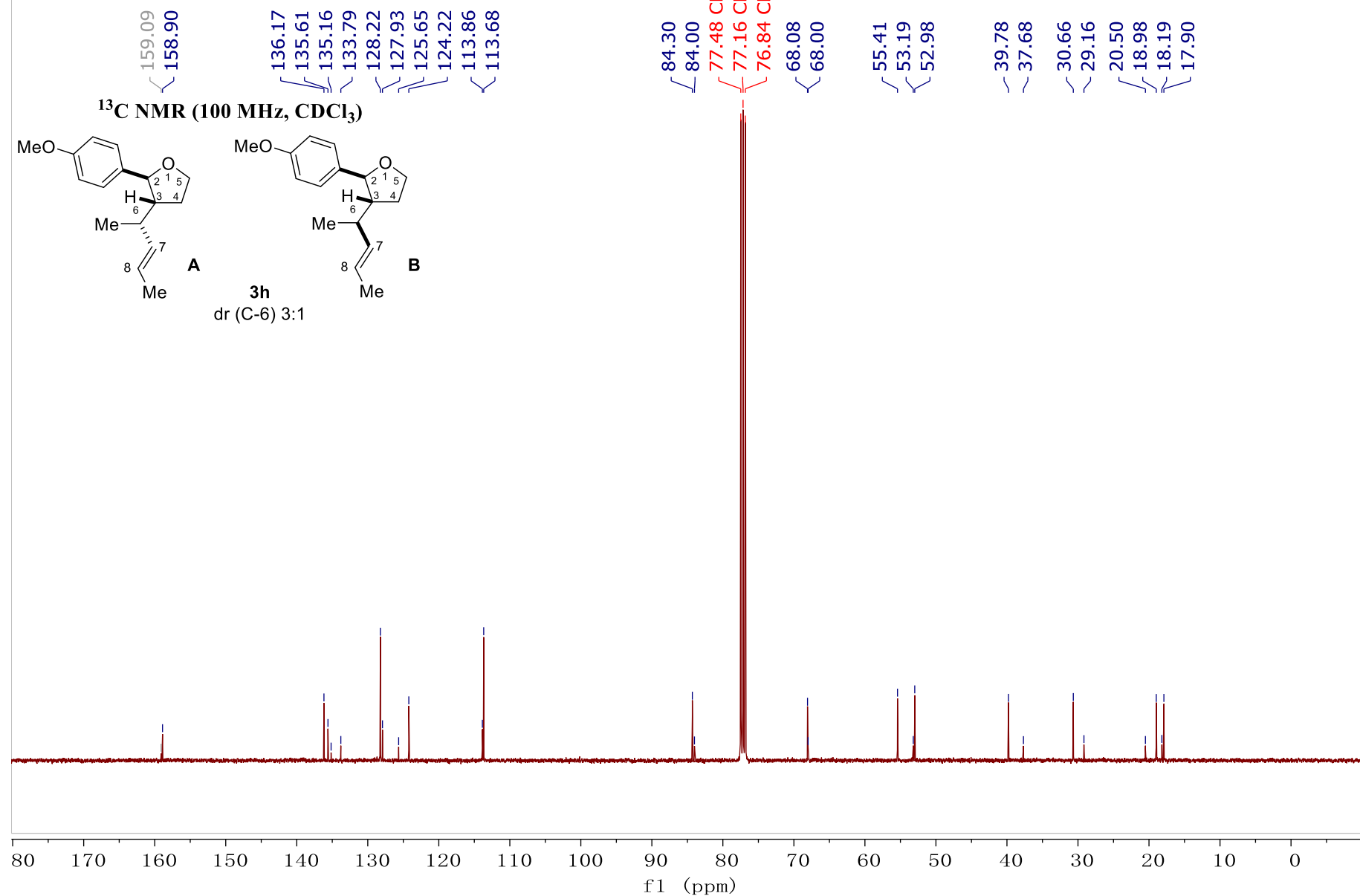
剀

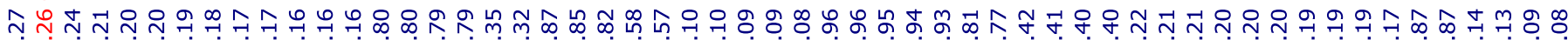

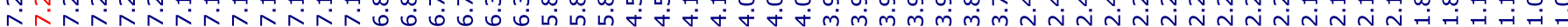

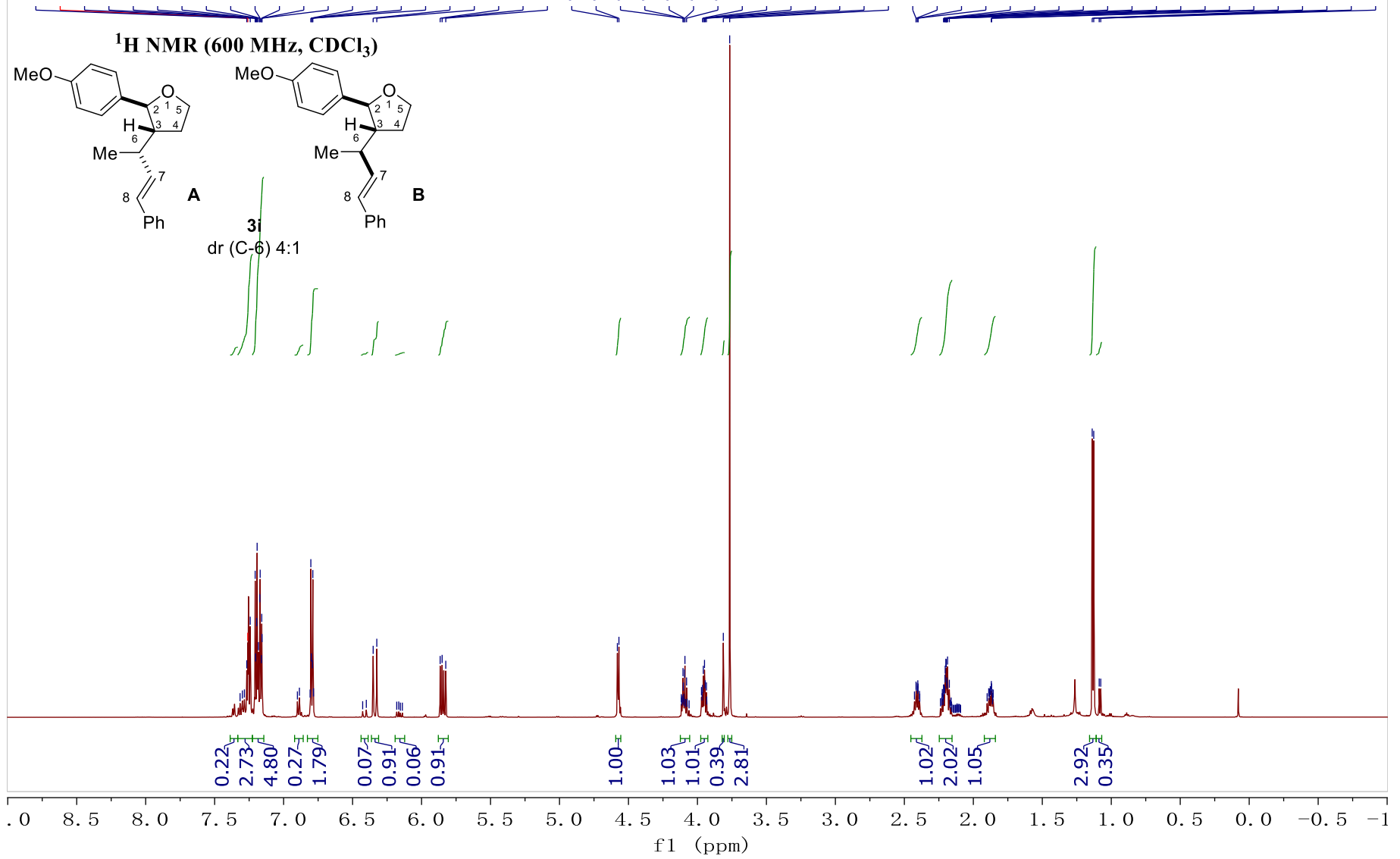

\section{บับ}

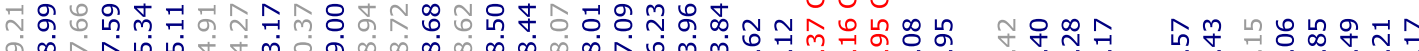

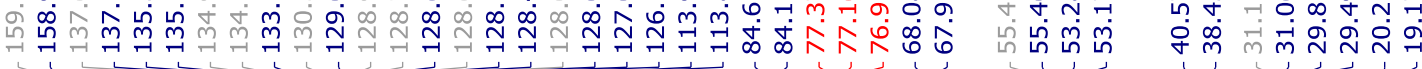
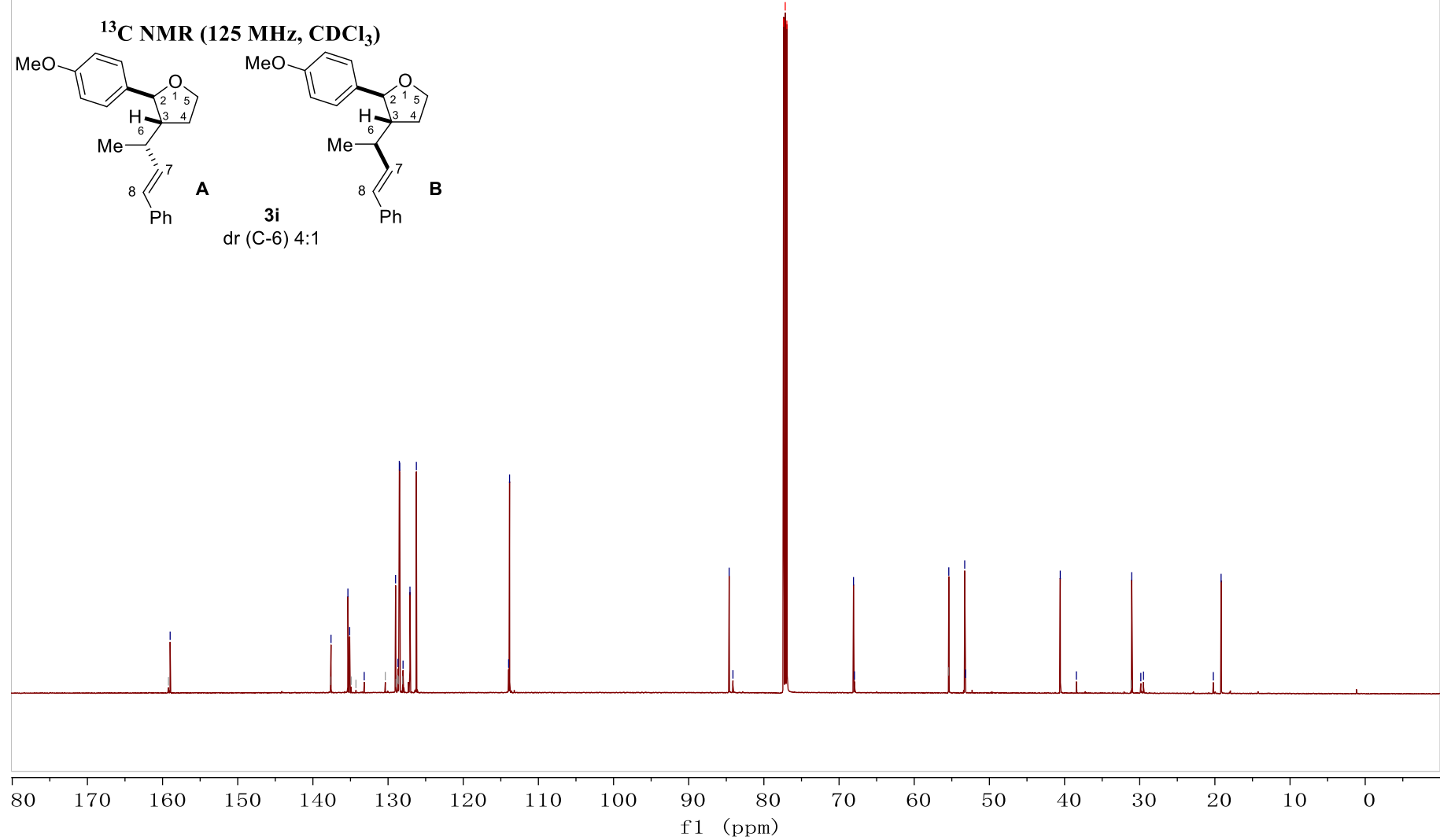
${ }^{1} \mathrm{H}-{ }^{1} \mathrm{H} \mathrm{COSY}$ - $90\left(600 \mathrm{MHz}, \mathrm{CDCl}_{3}\right)$

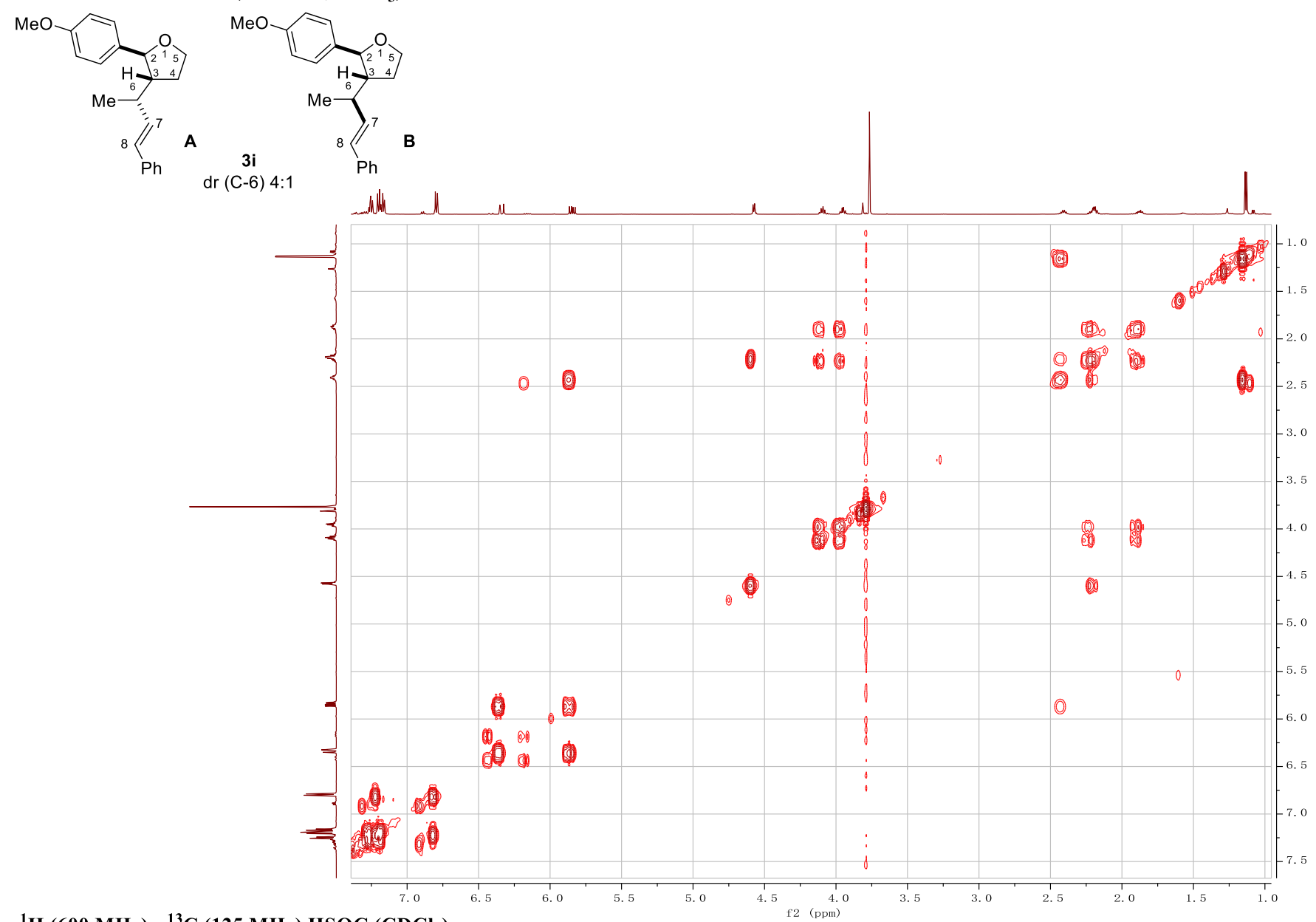

${ }^{1} \mathrm{H}(600 \mathrm{MHz})-{ }^{13} \mathrm{C}(125 \mathrm{MHz}) \mathrm{HSQC}\left(\mathrm{CDCl}_{3}\right)$

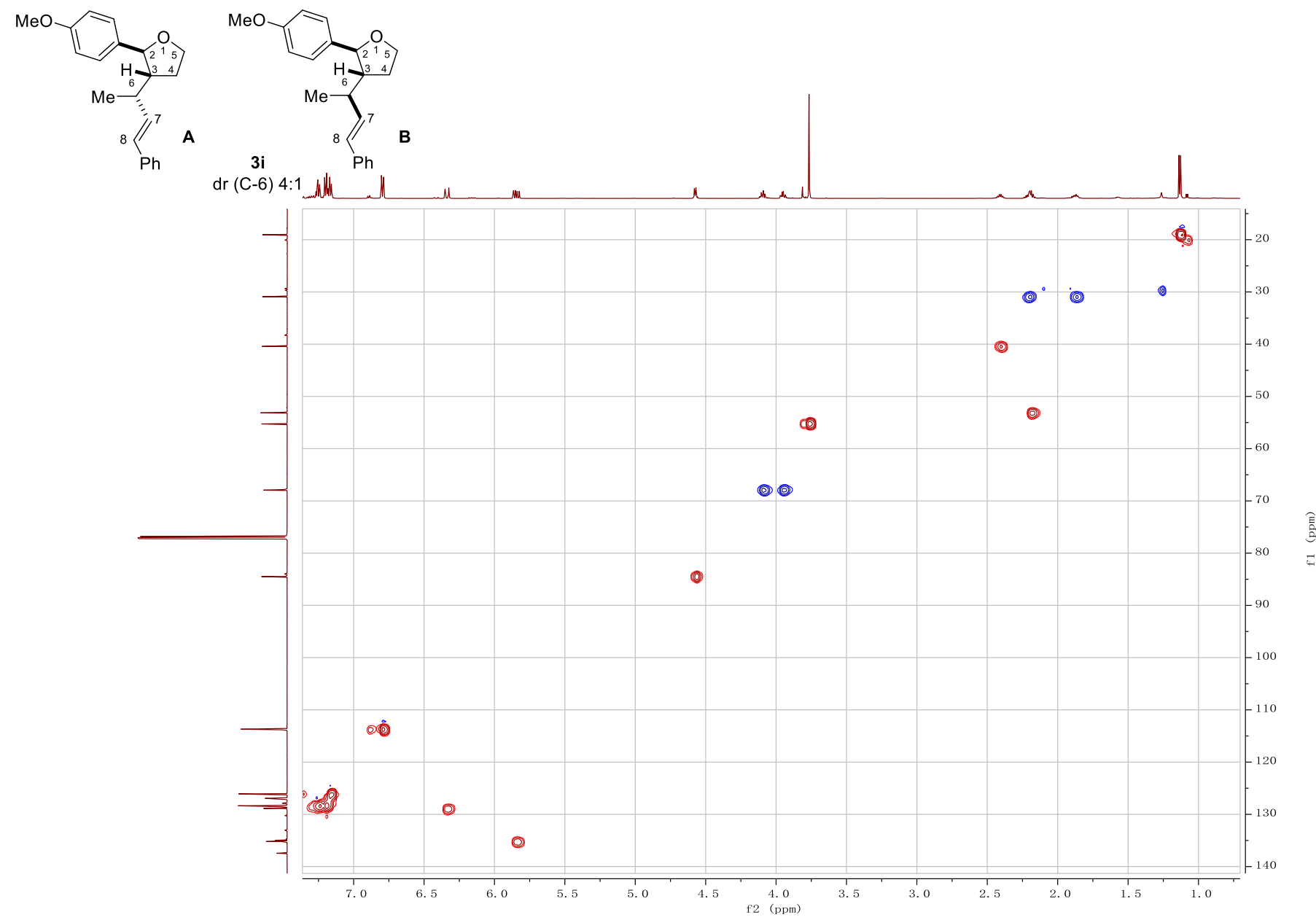




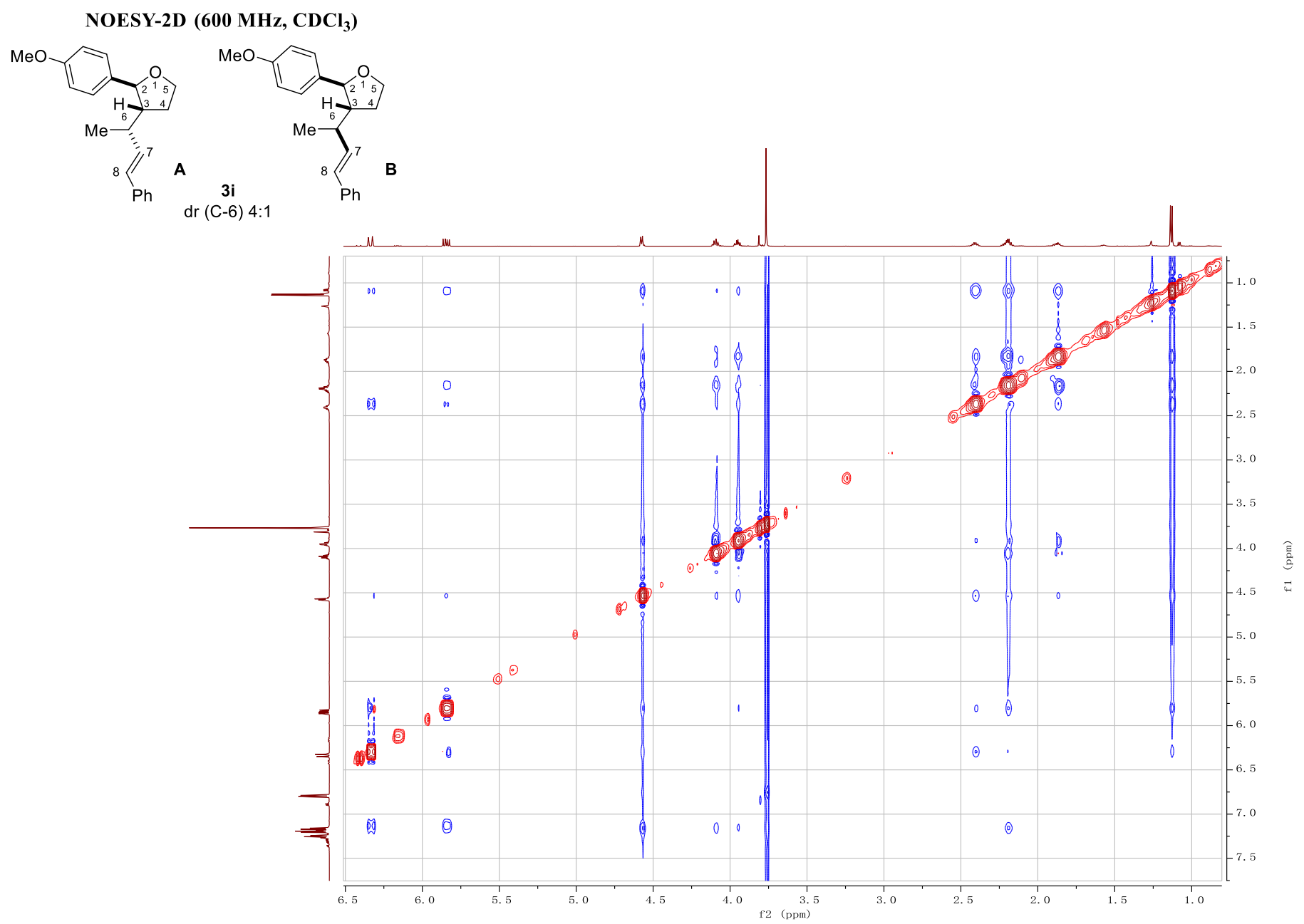

S29 


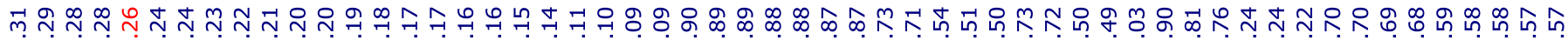
ヘ

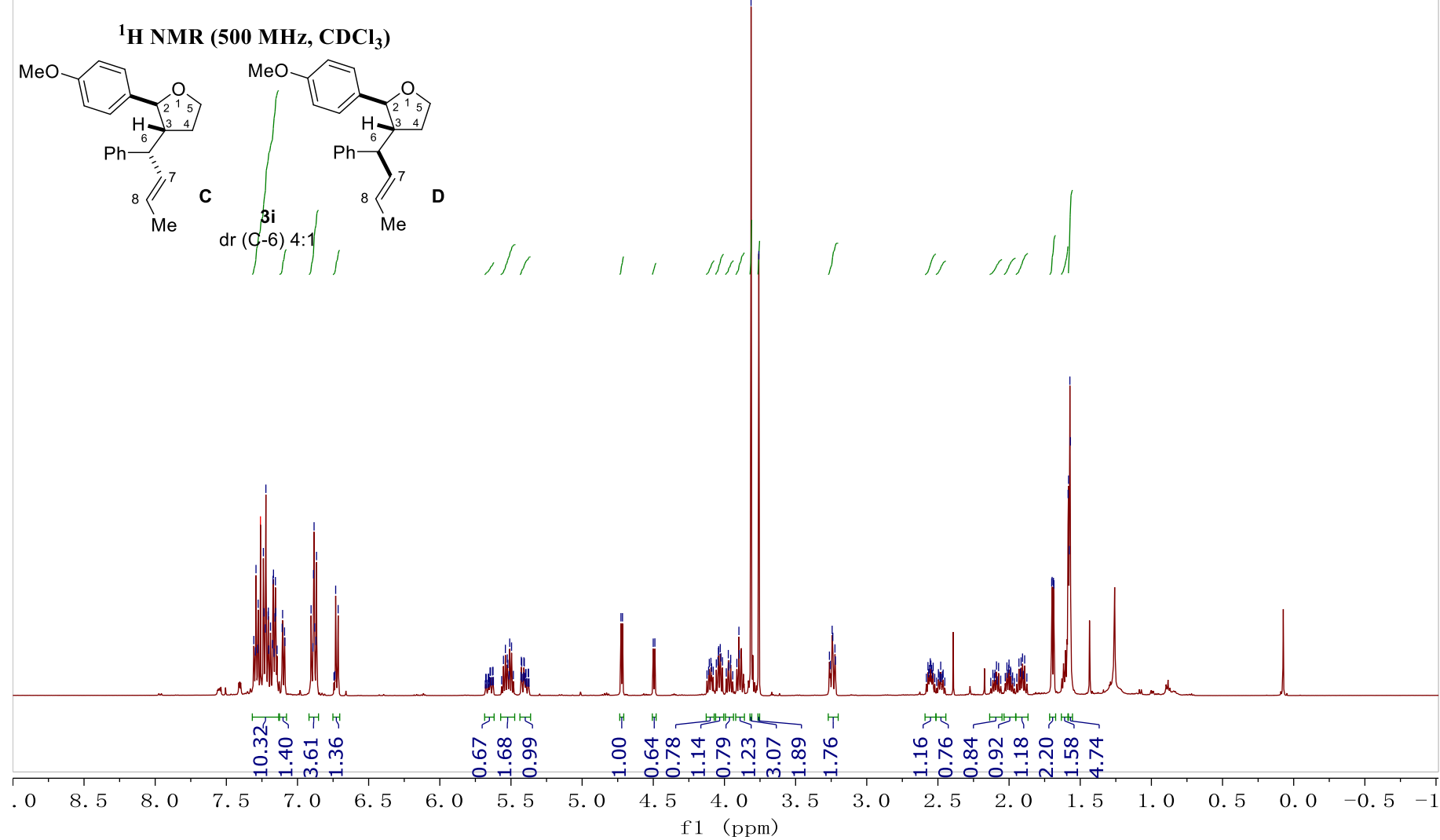

\section{는}

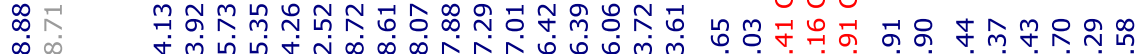

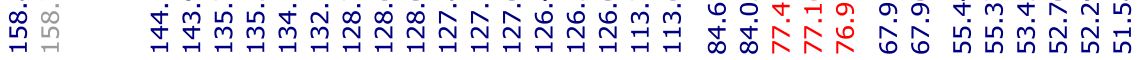

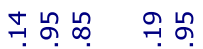

लंจ่ จิ

$1<$ W

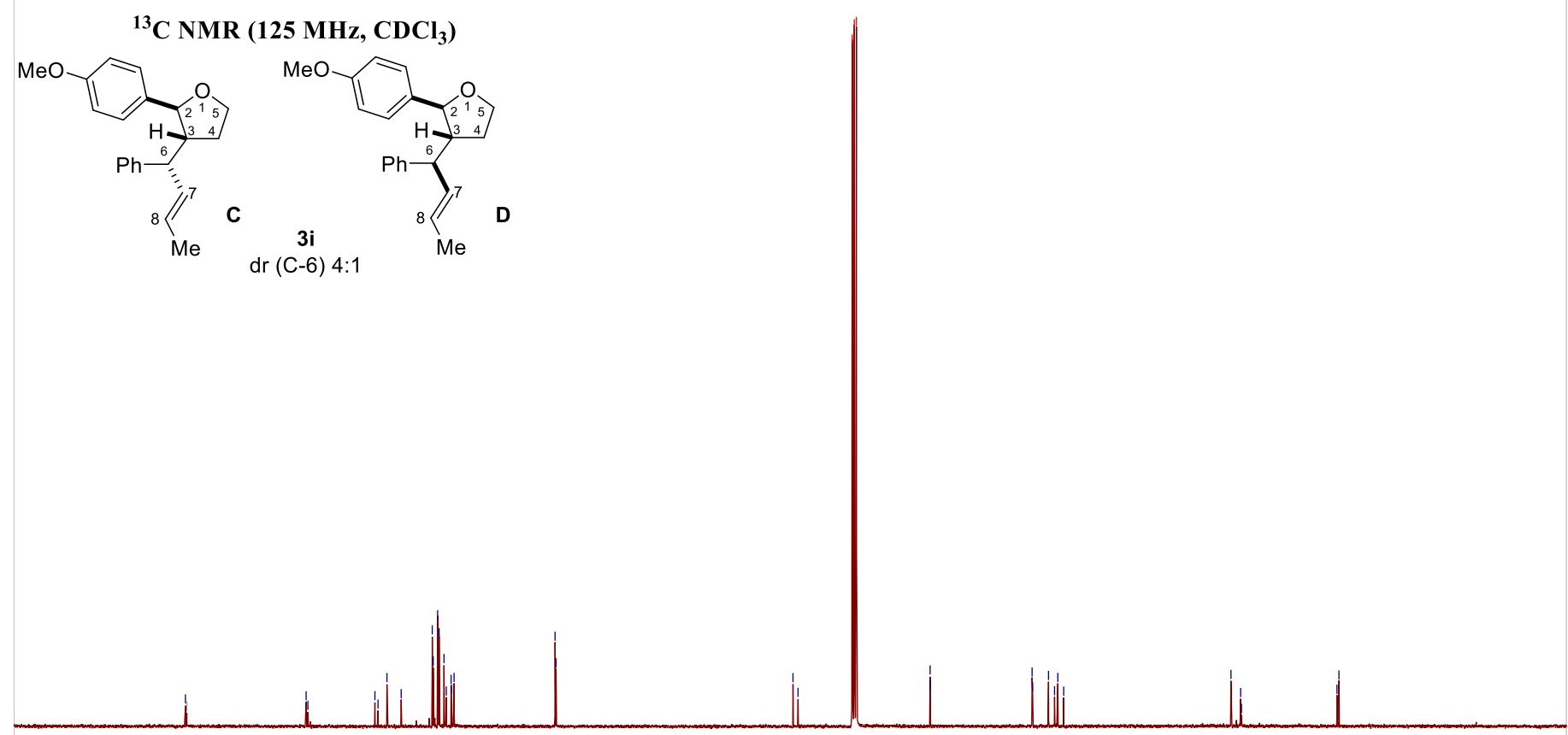


誉

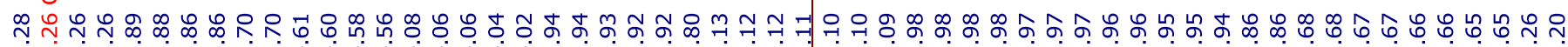

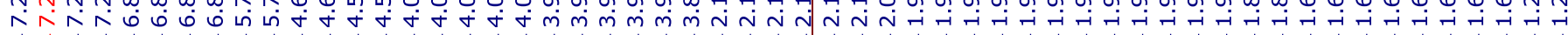

${ }^{1} \mathrm{H}$ NMR (400 MHz, $\left.\mathrm{CDCl}_{3}\right)$

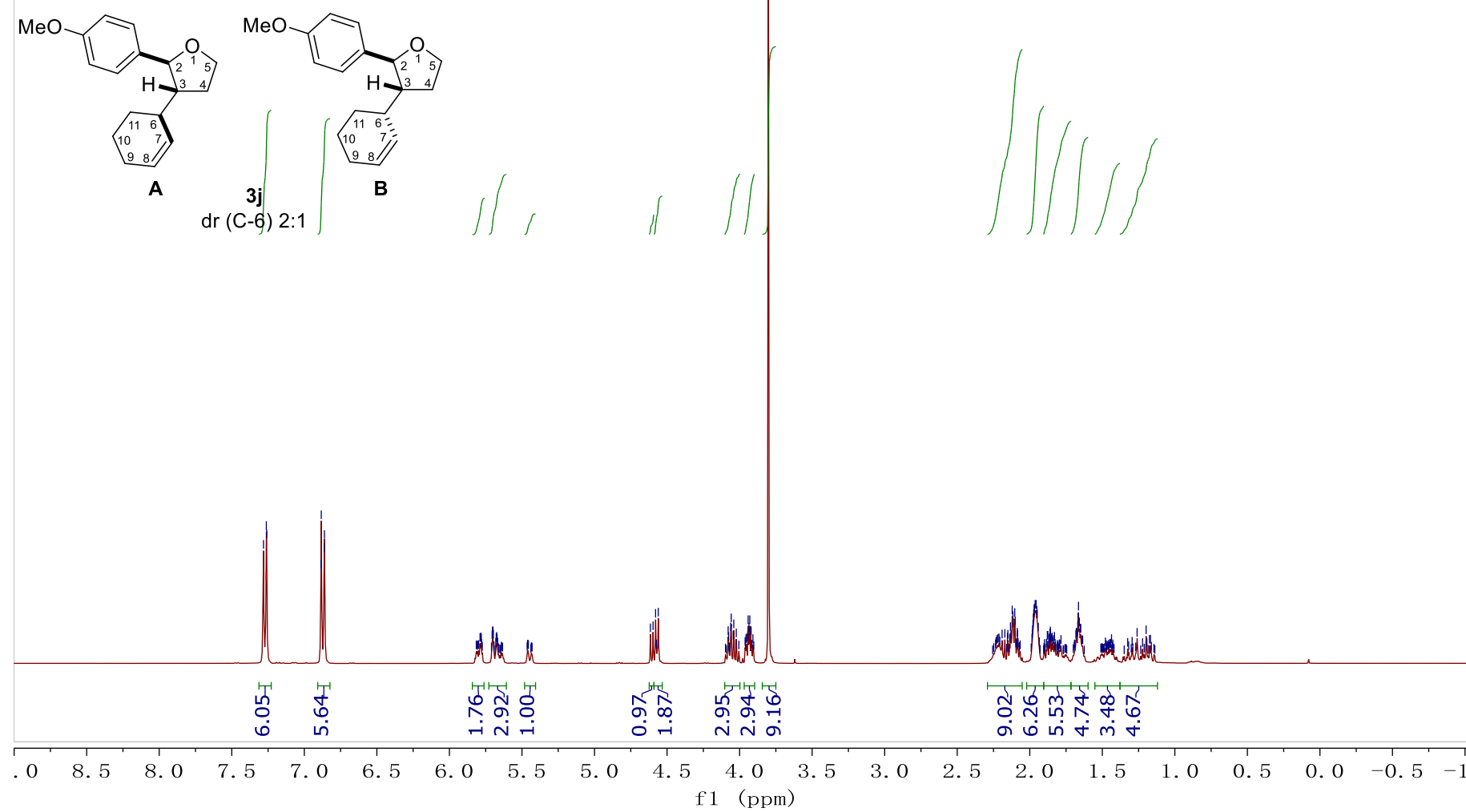

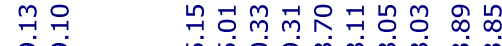

官品

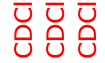

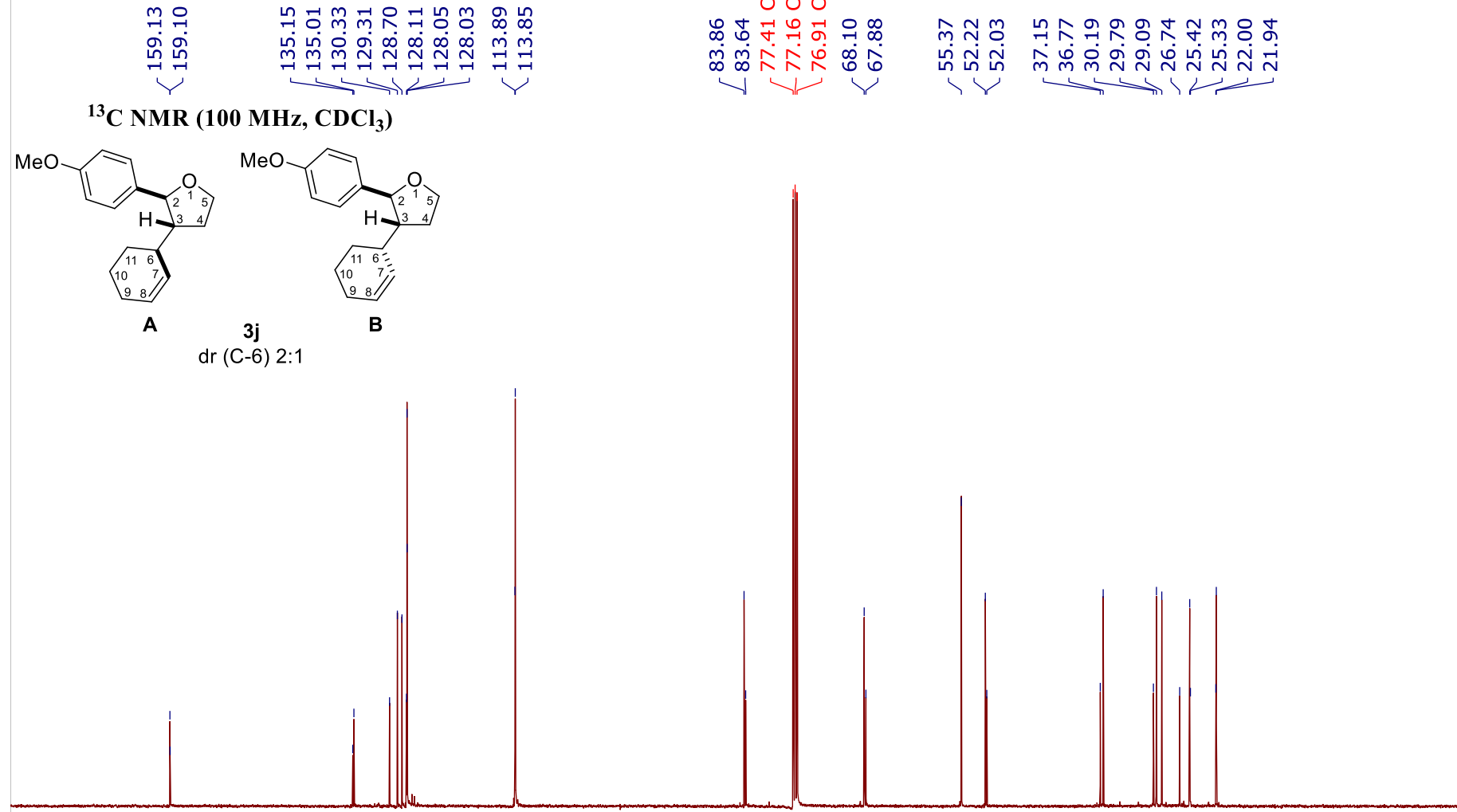

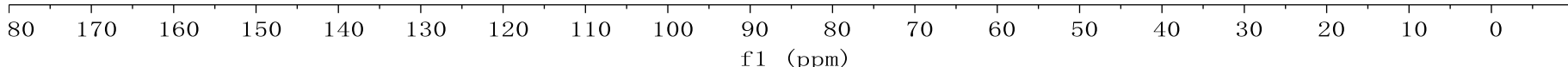


${ }^{1} \mathrm{H}$ NMR (400 MHz, $\mathrm{CDCl}_{3}$ )
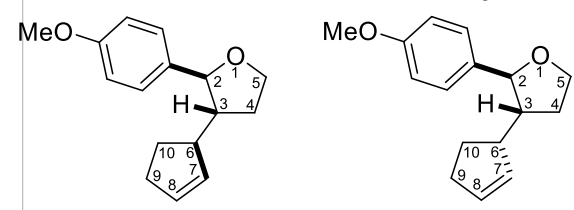

A $\quad \begin{aligned} & \mathbf{3 k} \\ & \mathrm{dr}(\mathrm{C}-6) \mathbf{3})\end{aligned}$

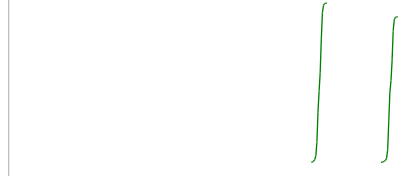

B
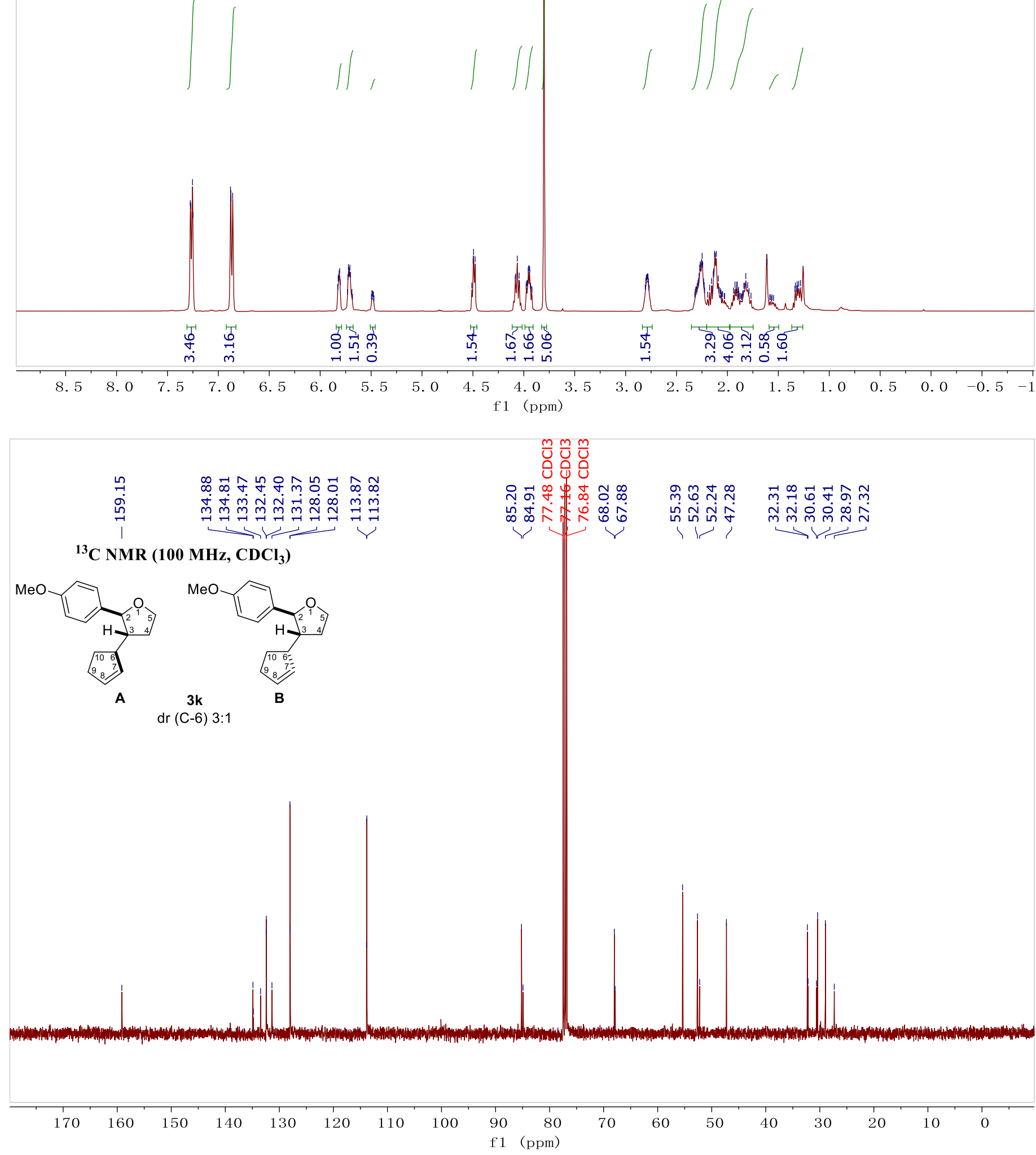
${ }^{1} \mathrm{H}$ NMR (500 MHz, $\mathrm{CDCl}_{3}$ )
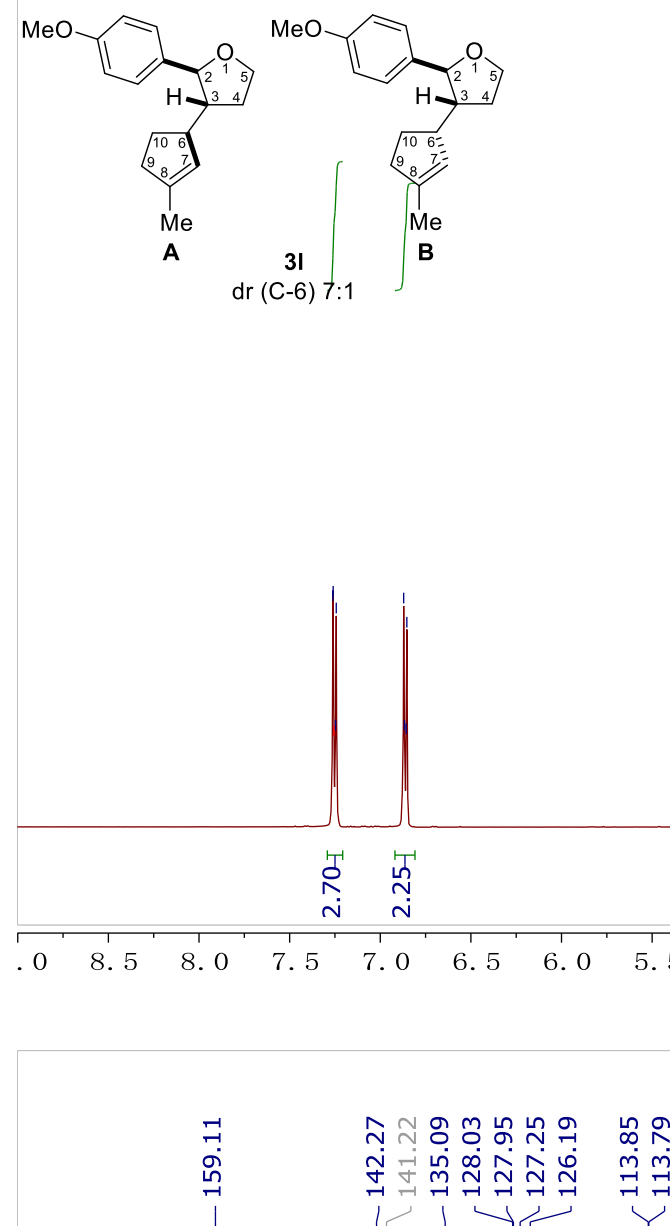

${ }^{13} \mathrm{C}$ NMR (125 MHz, $\left.\mathrm{CDCl}_{3}\right)$

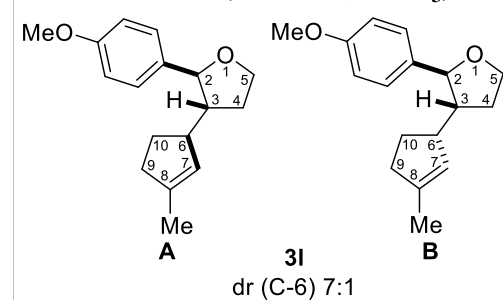

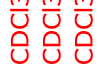

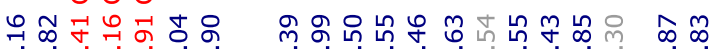

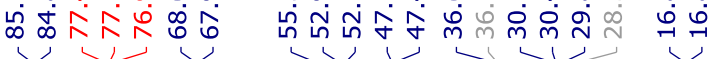




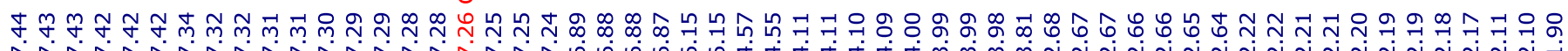

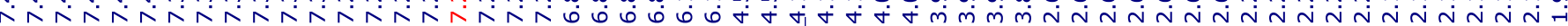

${ }^{1} \mathrm{H}$ NMR (500 MHz, $\left.\mathrm{CDCl}_{3}\right)$

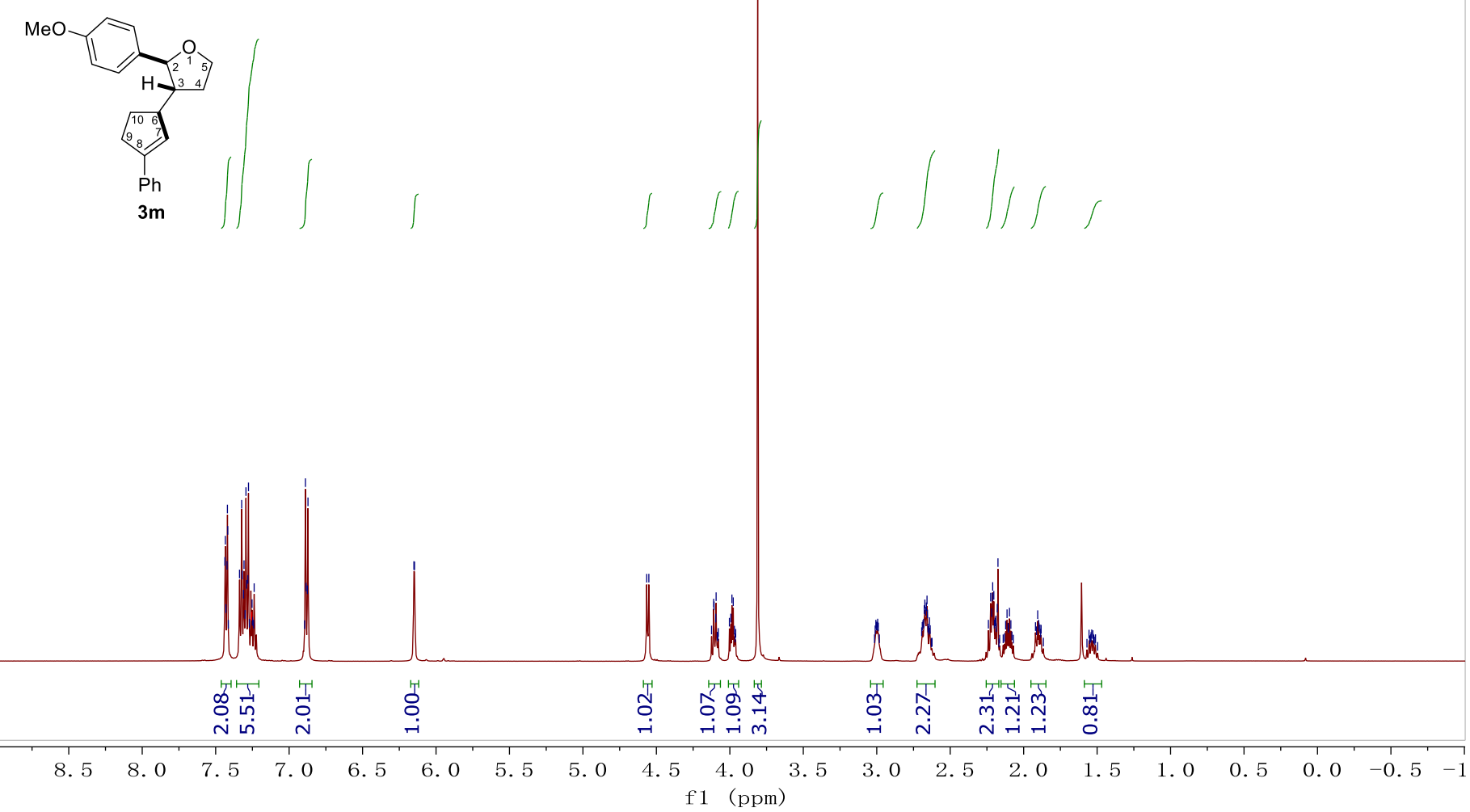

กิ $\quad \stackrel{\infty}{\sim}$ 于

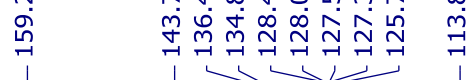

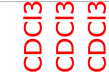

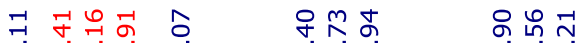

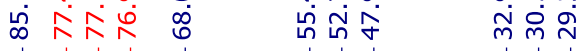

\section{${ }^{13} \mathrm{C}$ NMR (125 MHz, $\left.\mathrm{CDCl}_{3}\right)$}

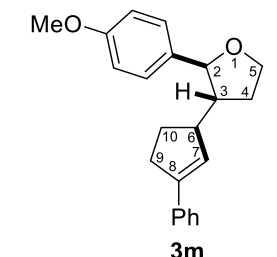

$3 \mathrm{~m}$
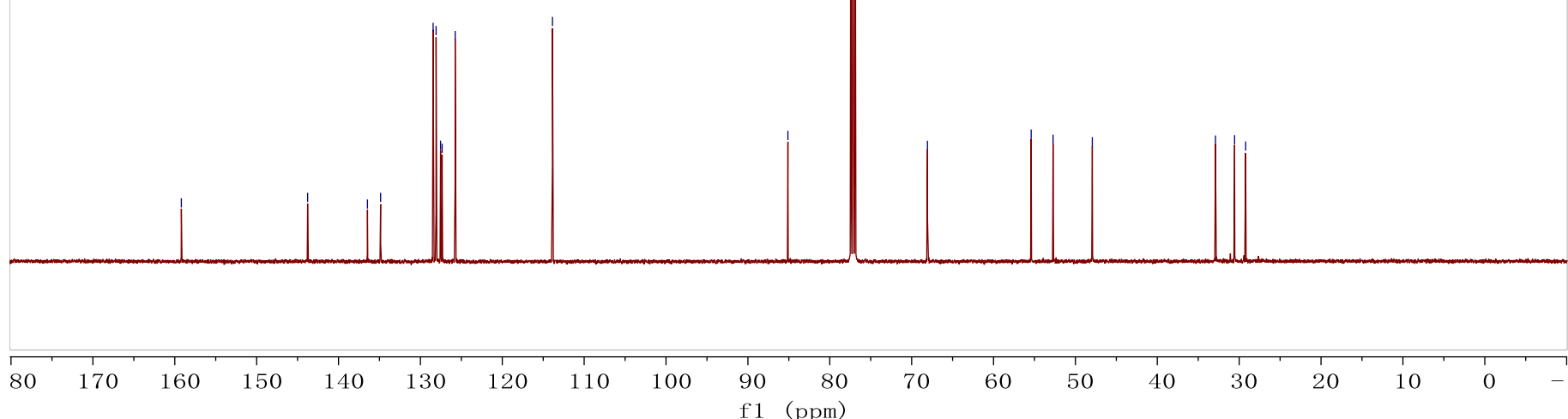

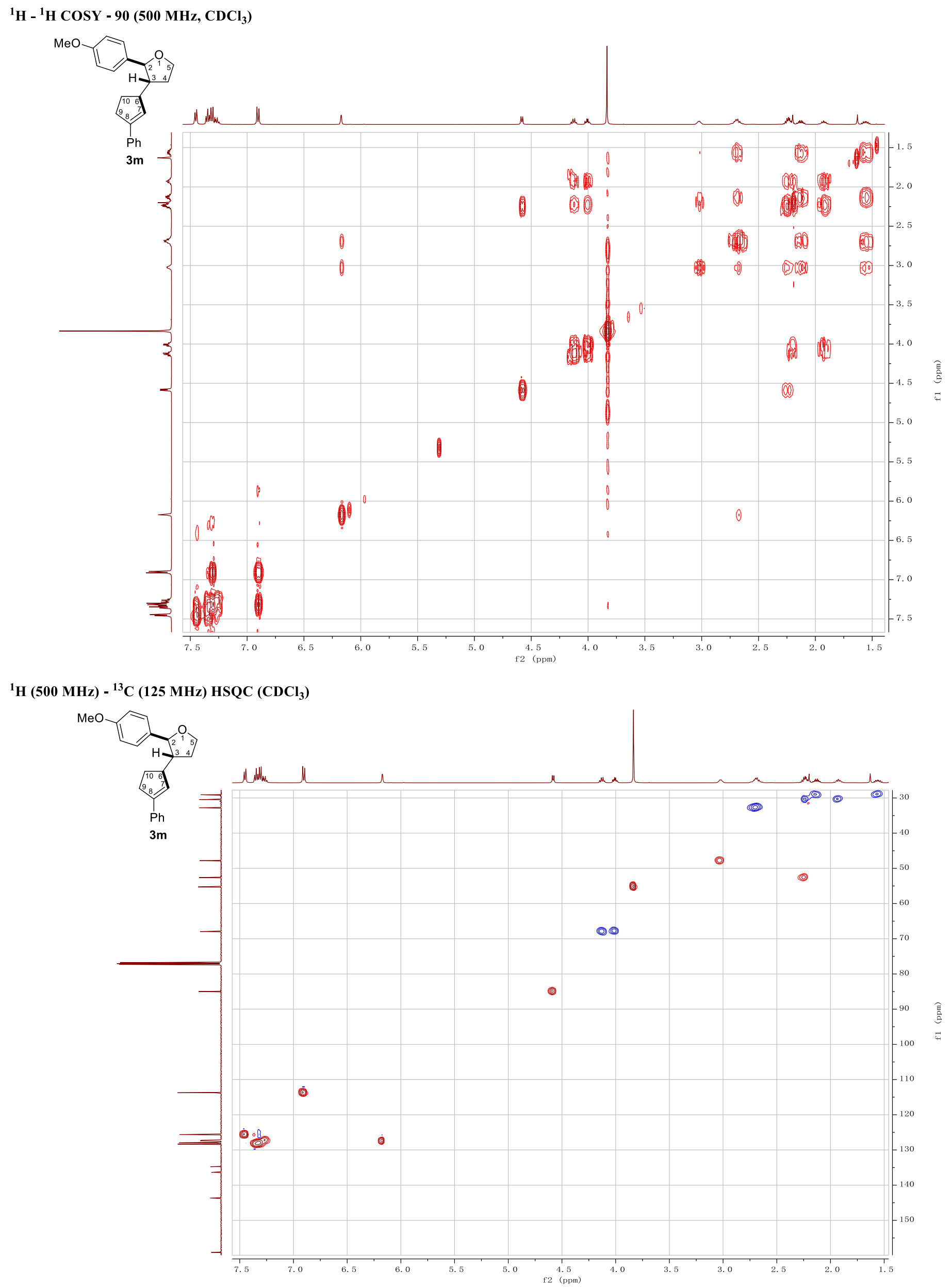

S35 
NOESY-2D (500 MHz, $\left.\mathrm{CDCl}_{3}\right)$

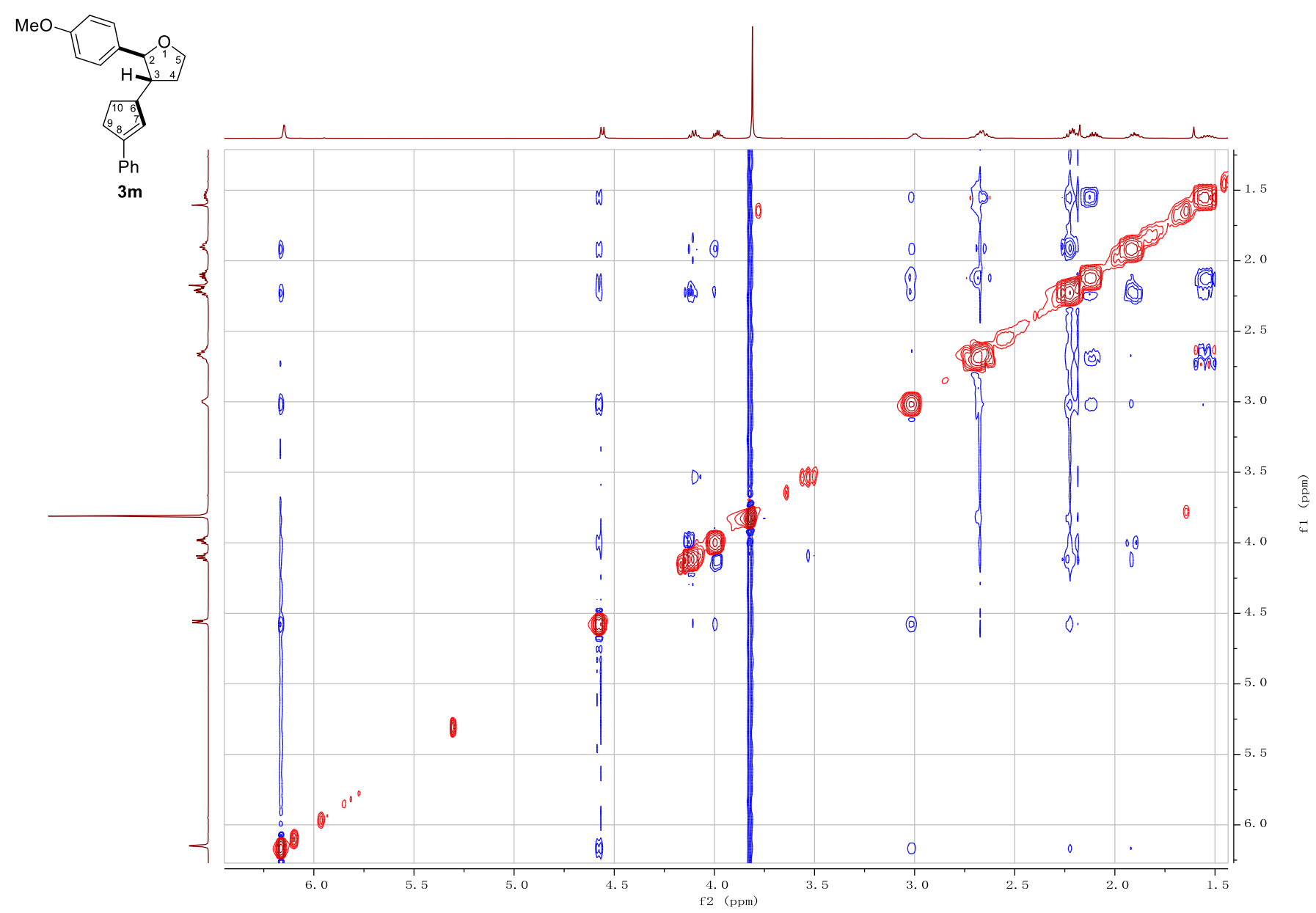




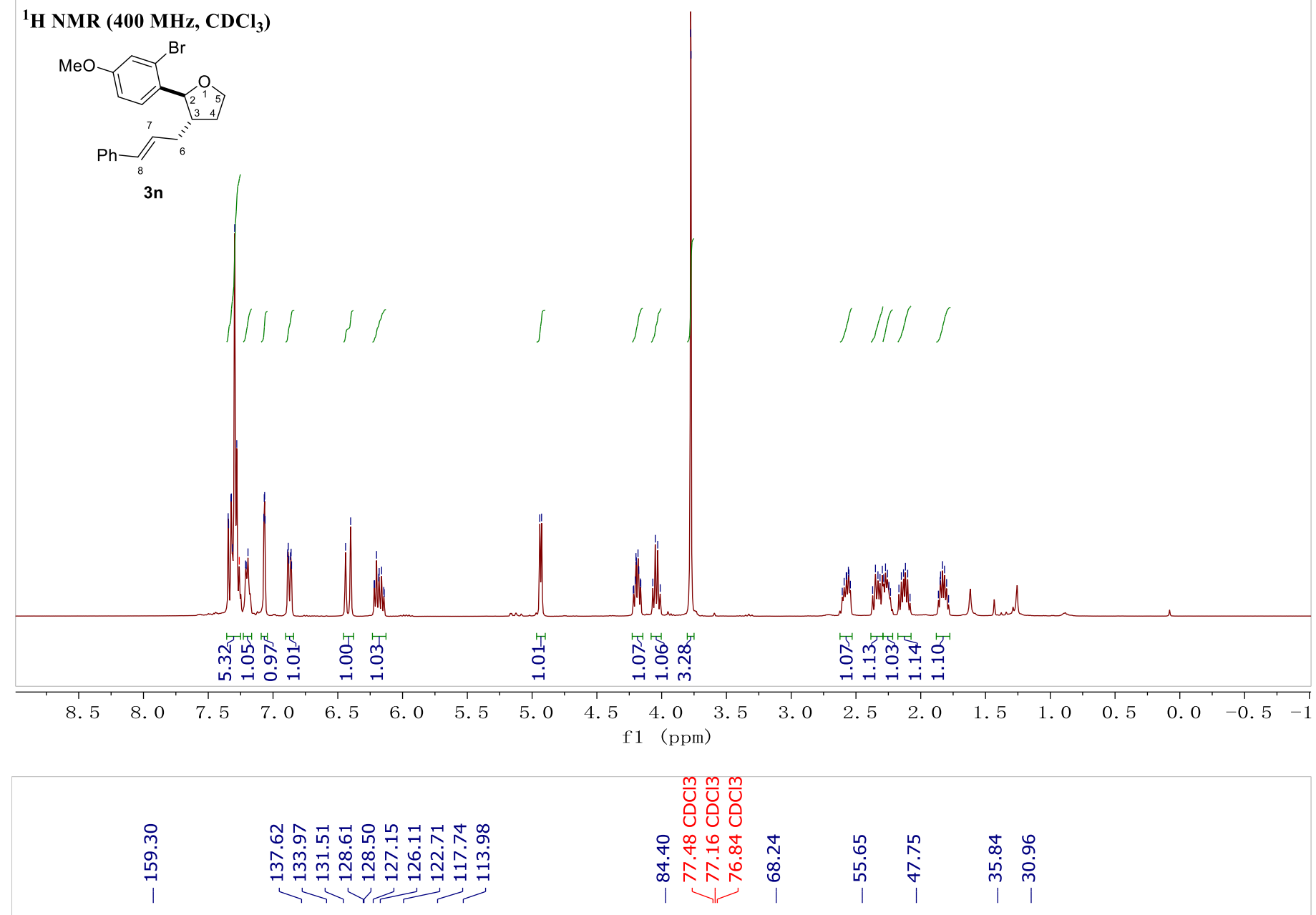

\section{${ }^{13} \mathrm{C}$ NMR (100 MHz, $\left.\mathrm{CDCl}_{3}\right)$}

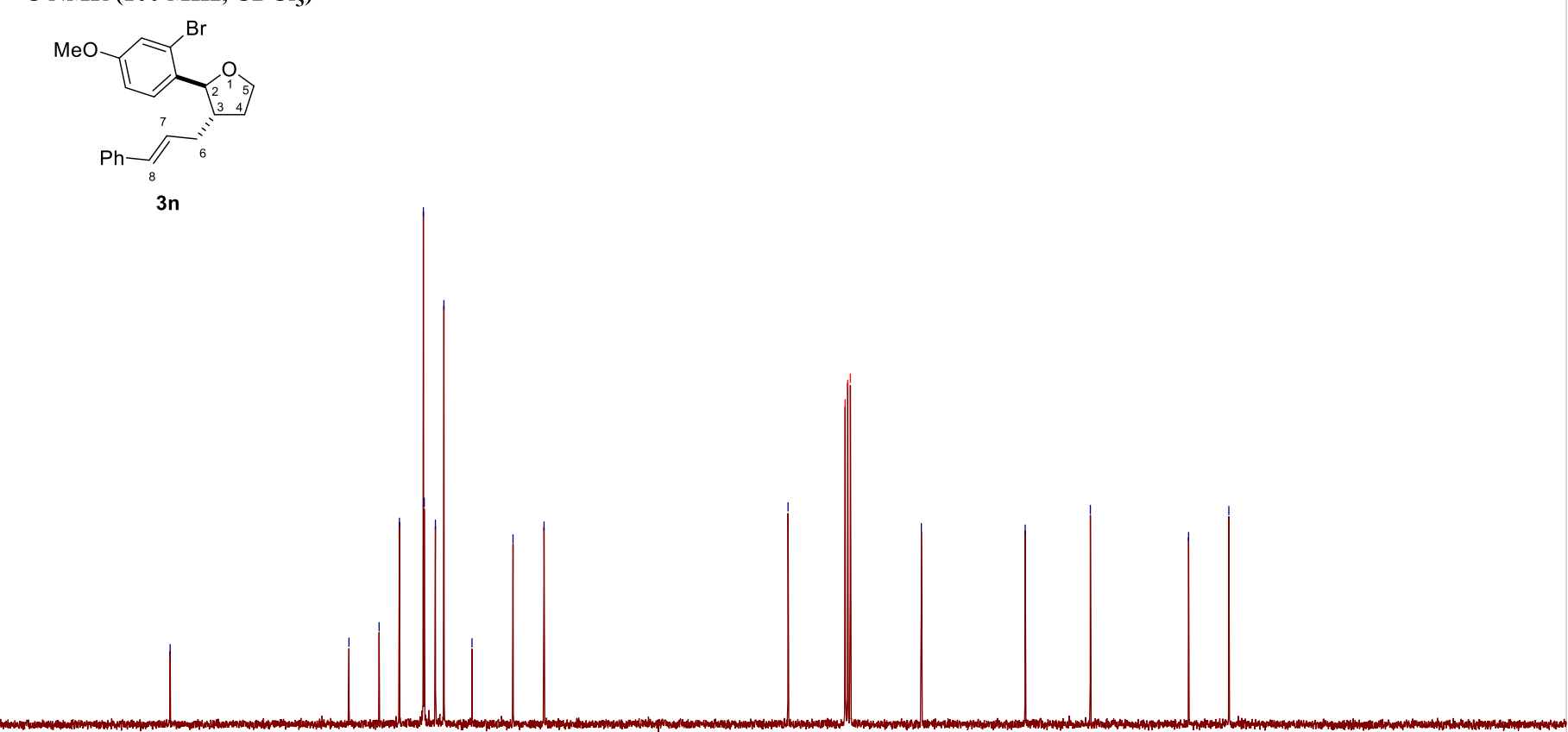




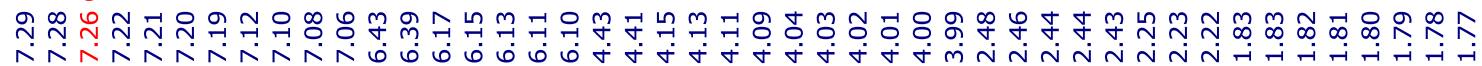

\section{${ }^{1} \mathrm{H}$ NMR (400 MHz, $\mathrm{CDCl}_{3}$ )}
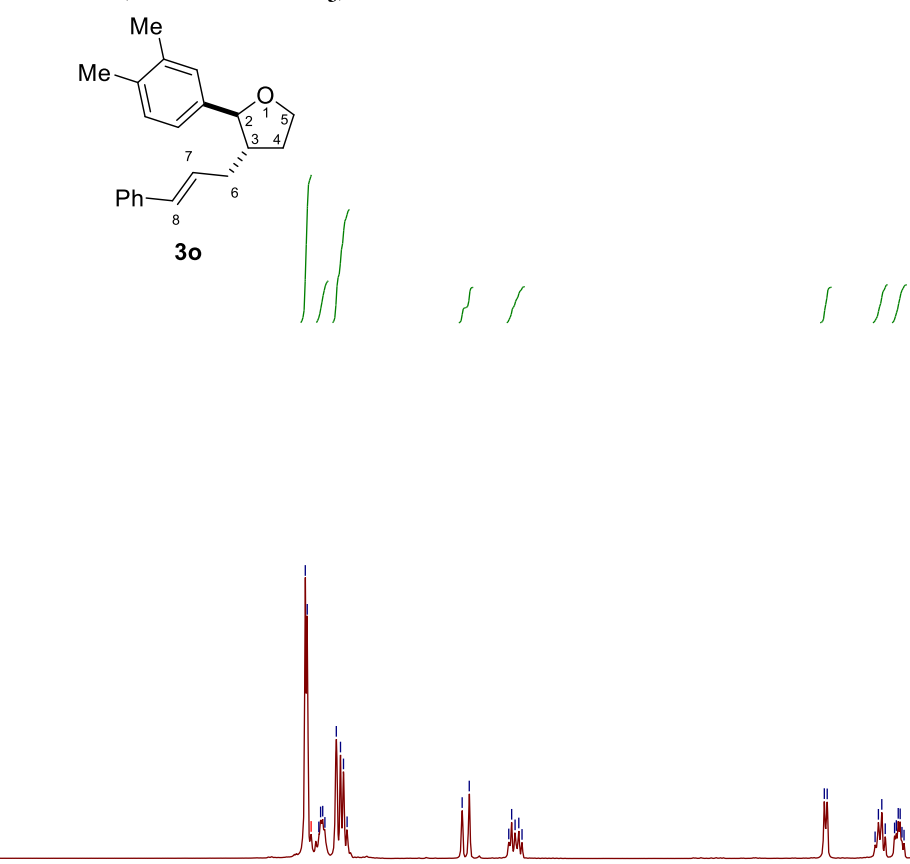

N
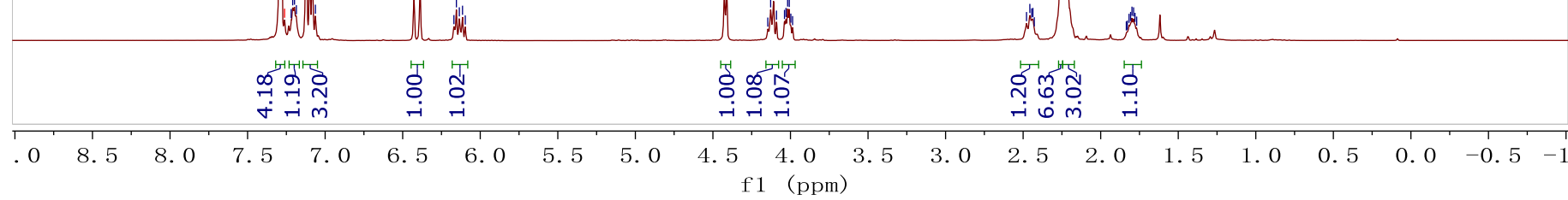

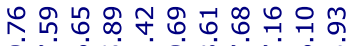

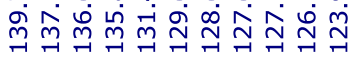

产商商

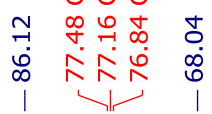

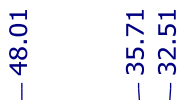

هั ซุర

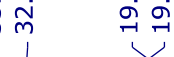

\section{${ }^{13} \mathrm{C}$ NMR (100 MHz, $\left.\mathrm{CDCl}_{3}\right)$}

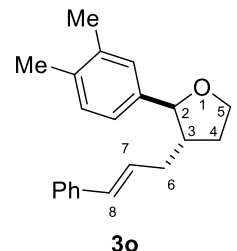

30

$\begin{array}{llll}170 & 160 & 150 & 140\end{array}$

$130 \quad 120$

$110 \quad 100$

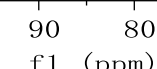

70

60

50

$40 \quad 30$

20 
${ }^{1}$ H NMR (400 MHz, $\mathrm{CDCl}_{3}$ )

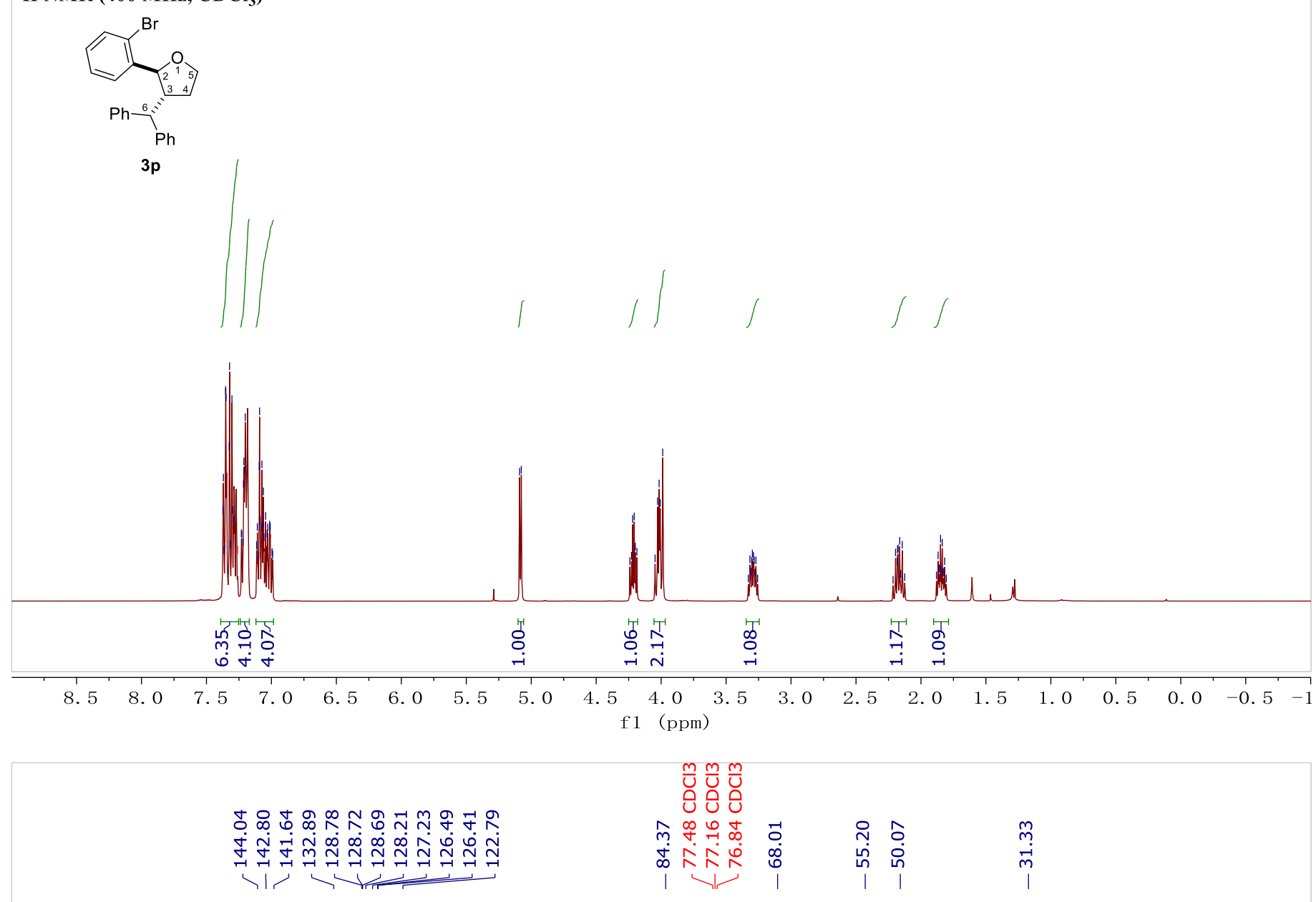

${ }^{13} \mathrm{C}$ NMR (100 MHz, $\left.\mathrm{CDCl}_{3}\right)$

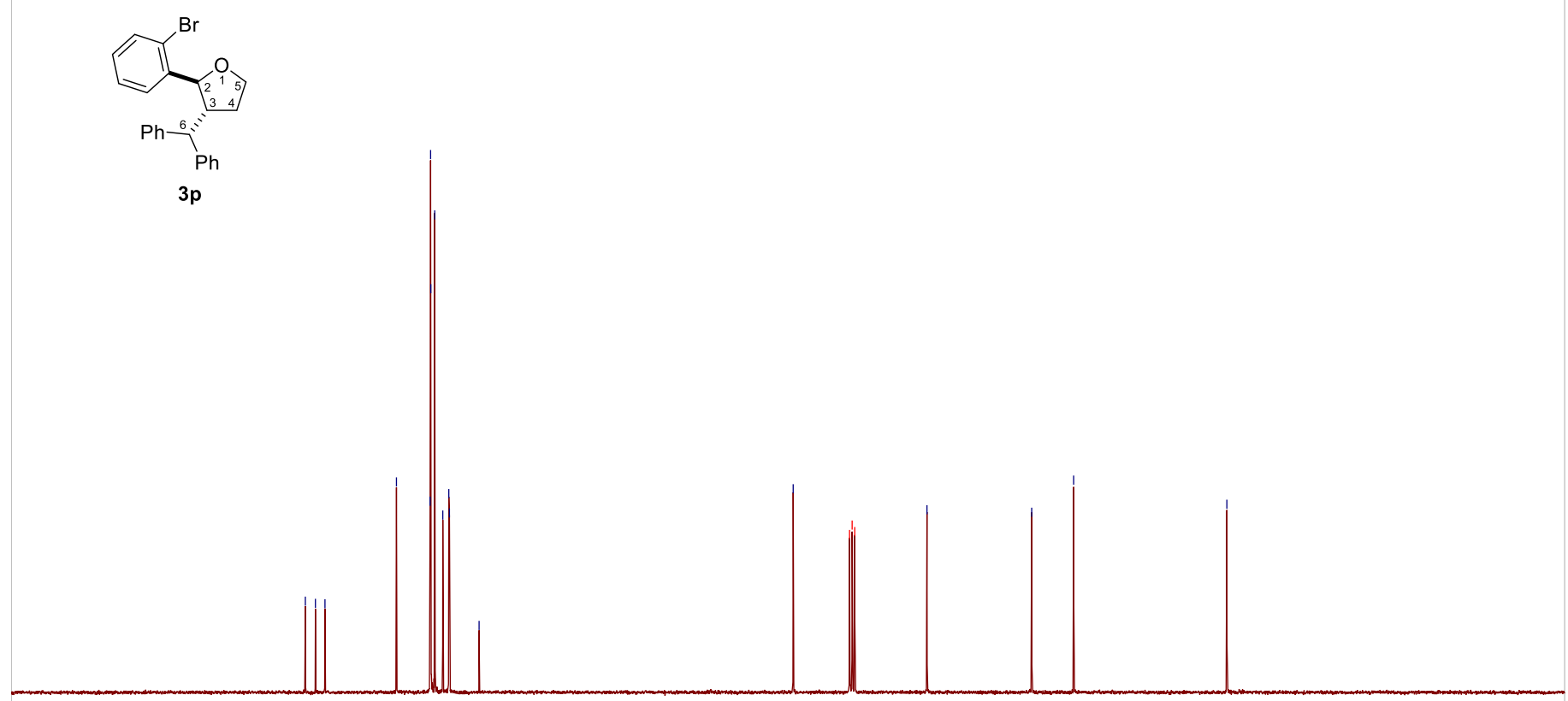


${ }^{1} \mathrm{H}$ NMR (400 MHz, $\left.\mathrm{CDCl}_{3}\right)$
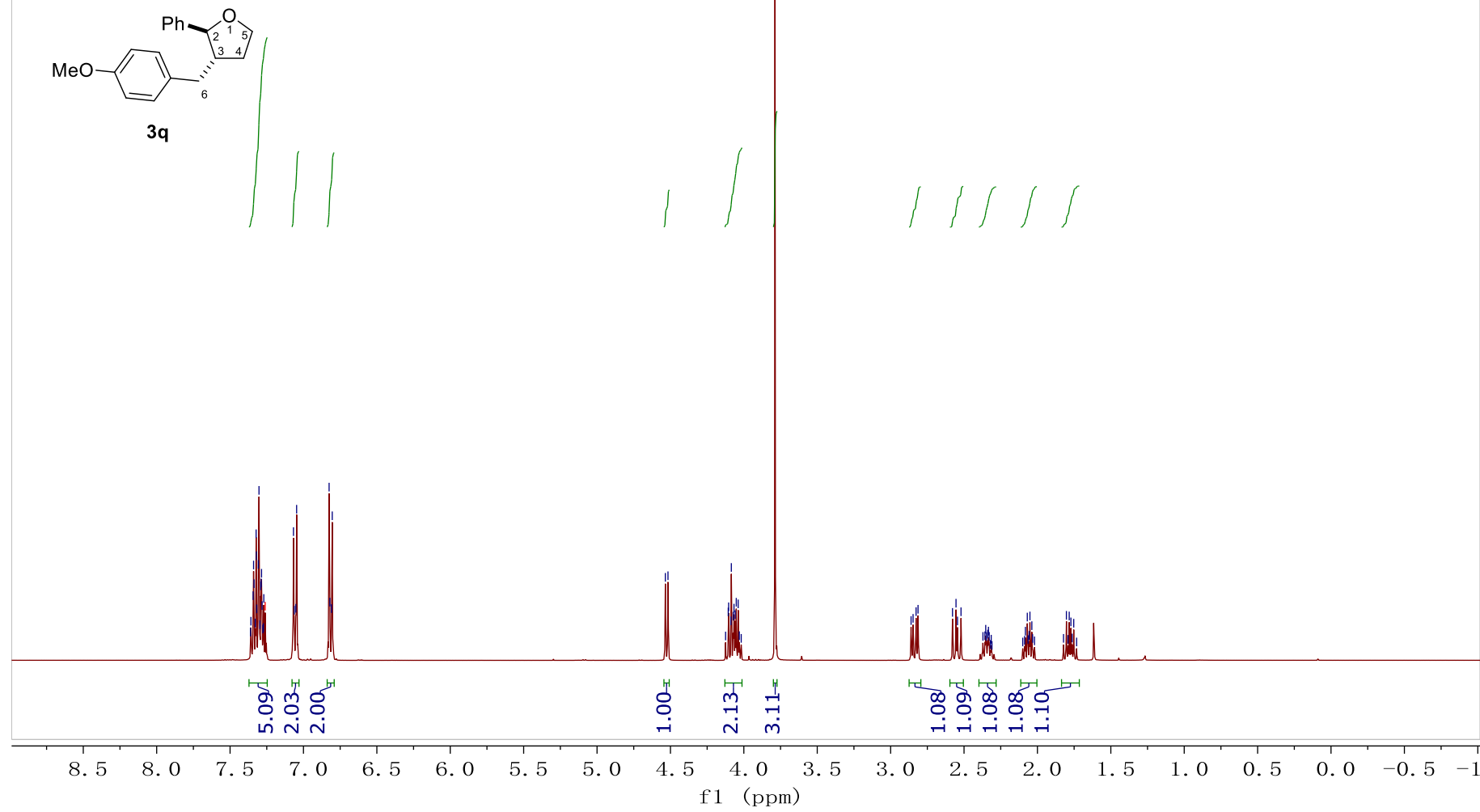

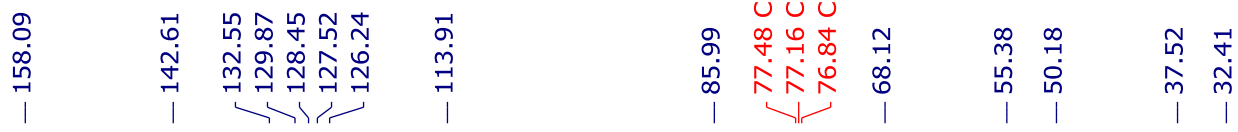

${ }^{13} \mathrm{C}$ NMR (100 MHz, $\left.\mathrm{CDCl}_{3}\right)$

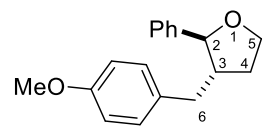

$3 q$

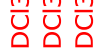
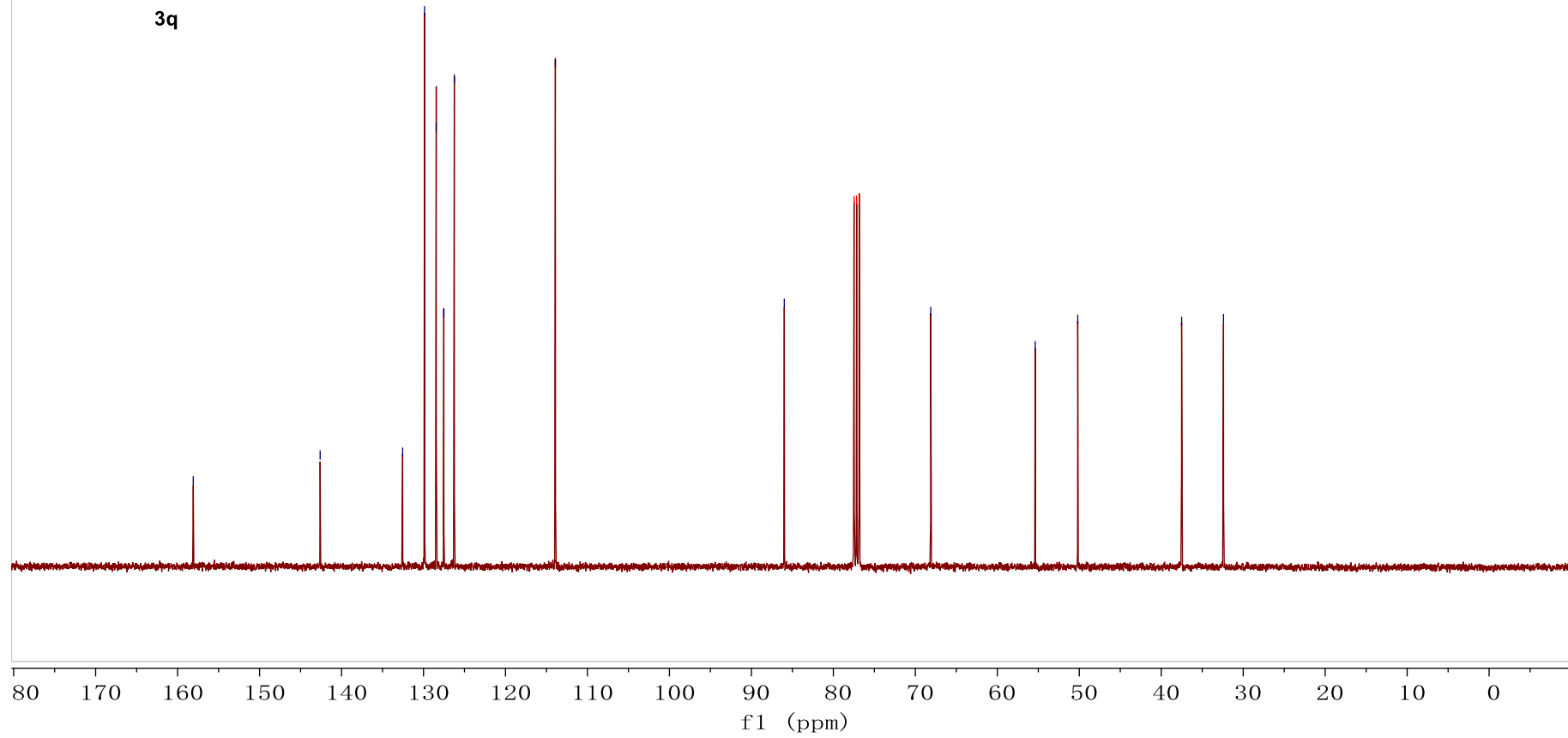
${ }^{1} \mathrm{H}$ NMR (400 MHz, $\left.\mathrm{CDCl}_{3}\right)$

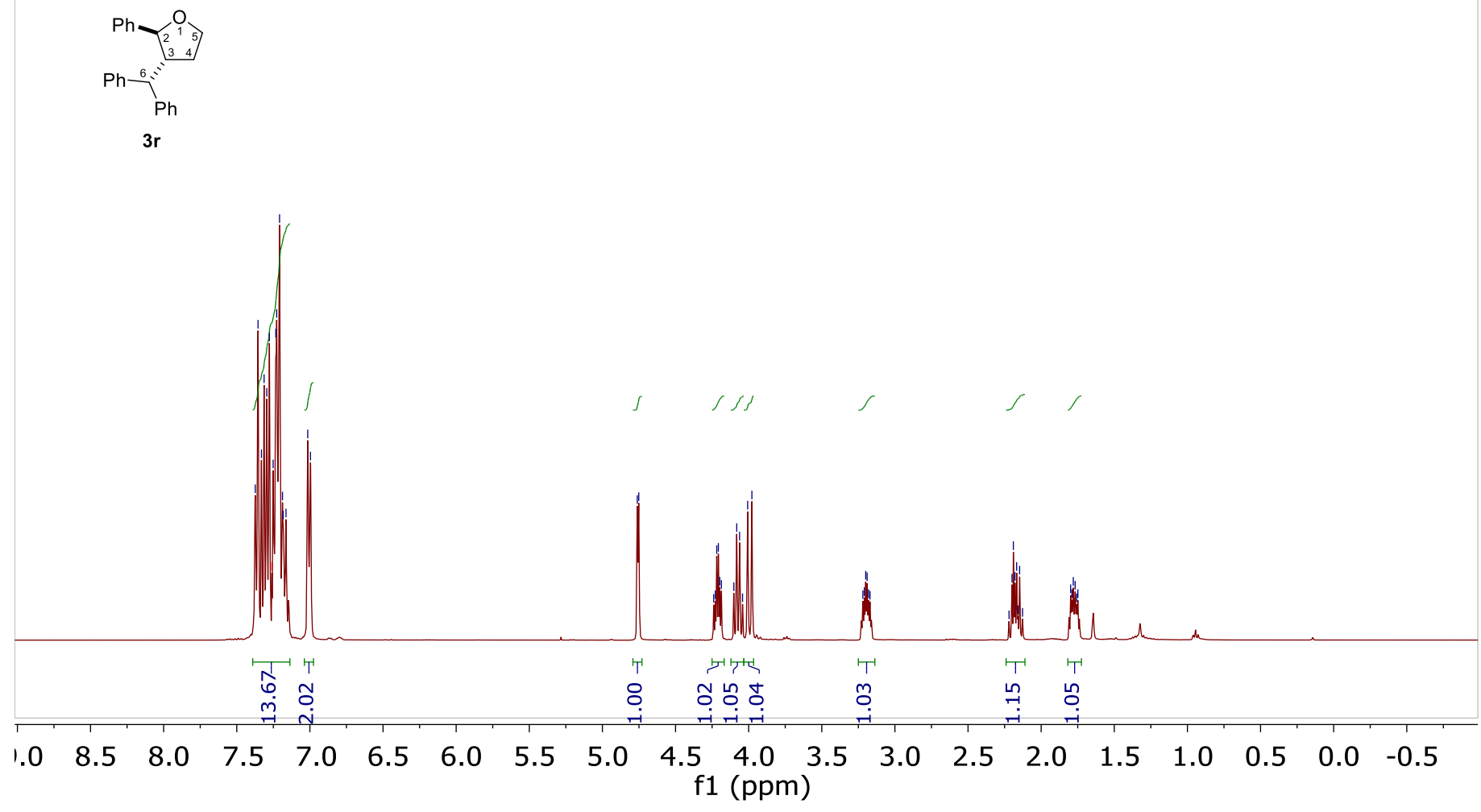

$3 r$

${ }^{13} \mathrm{C}$ NMR (100 MHz, $\left.\mathrm{CDCl}_{3}\right)$

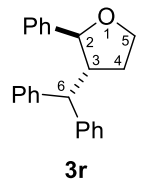

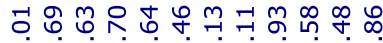

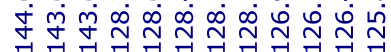
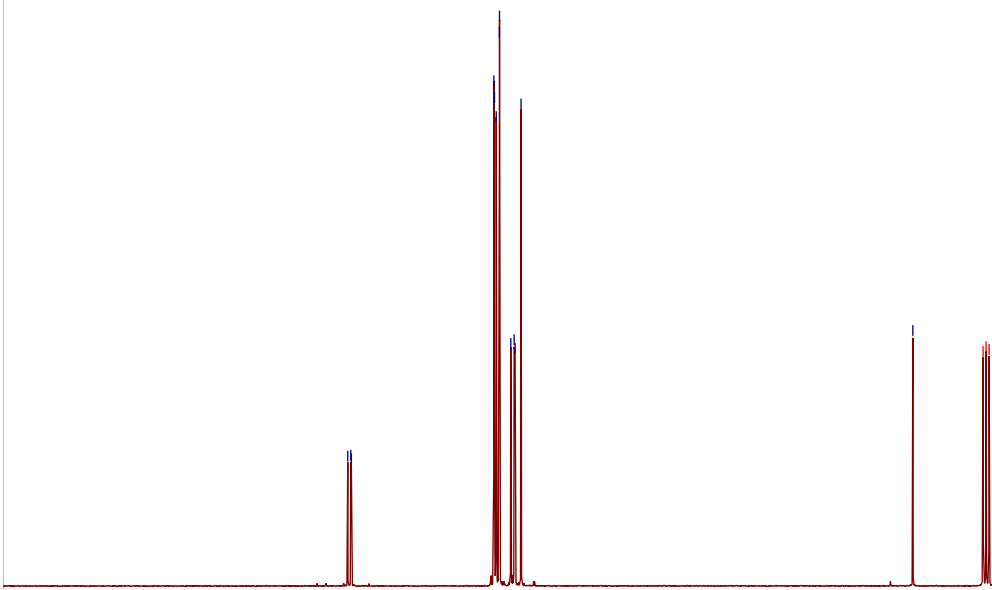

80

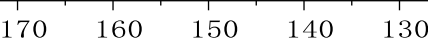

120 


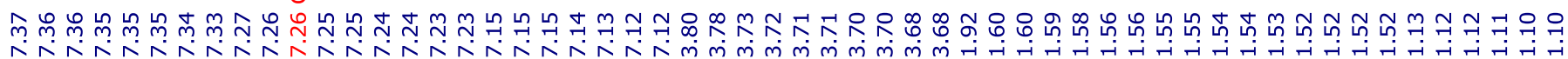

\section{${ }^{1} \mathrm{H}$ NMR (500 MHz, $\mathrm{CDCl}_{3}$ )}

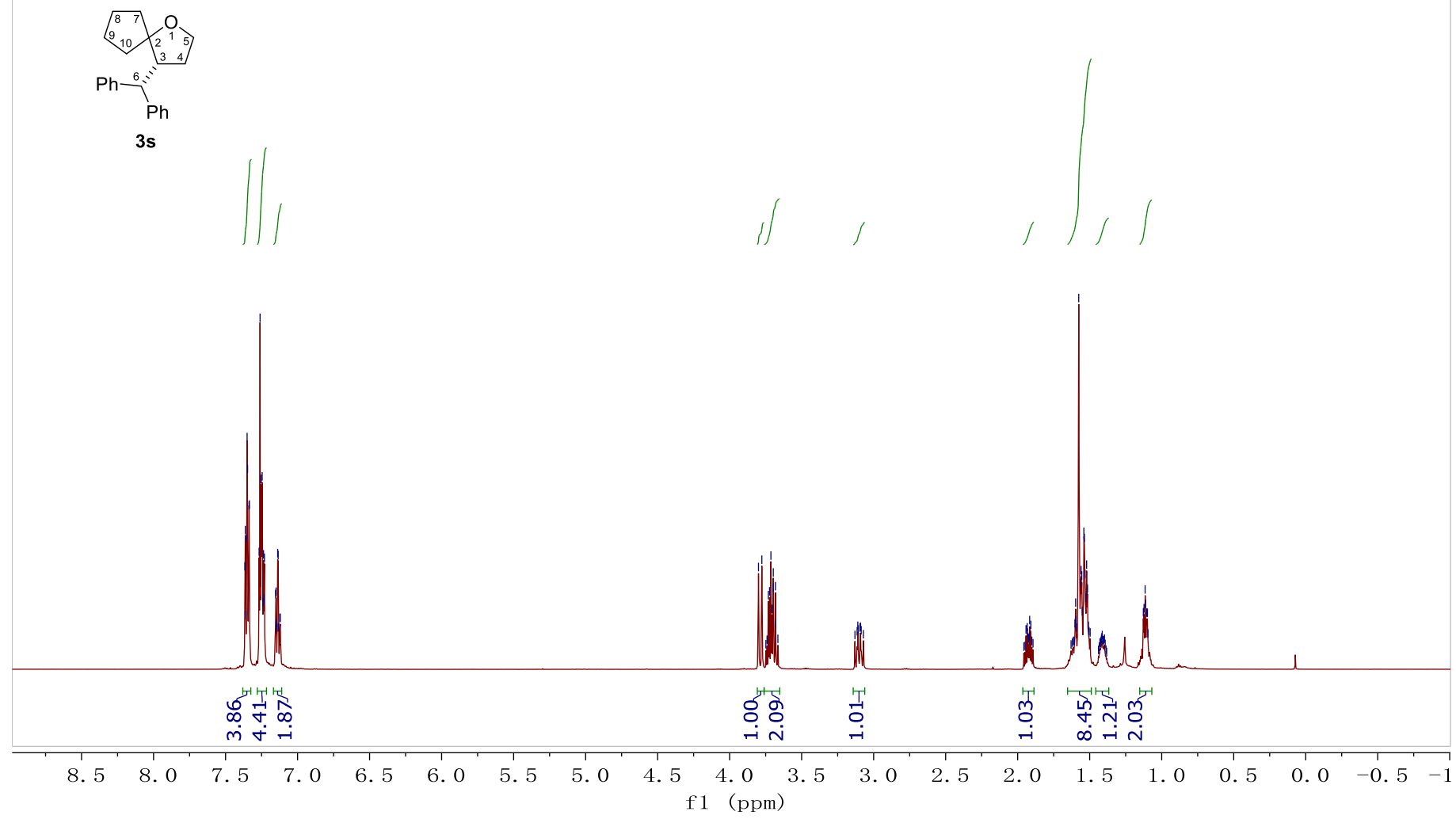

\section{${ }^{13} \mathrm{C}$ NMR (125 MHz, $\left.\mathrm{CDCl}_{3}\right)$}

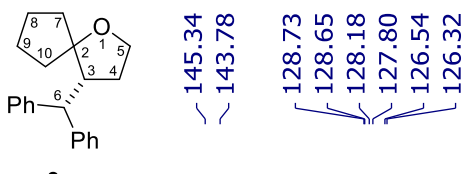

3 s
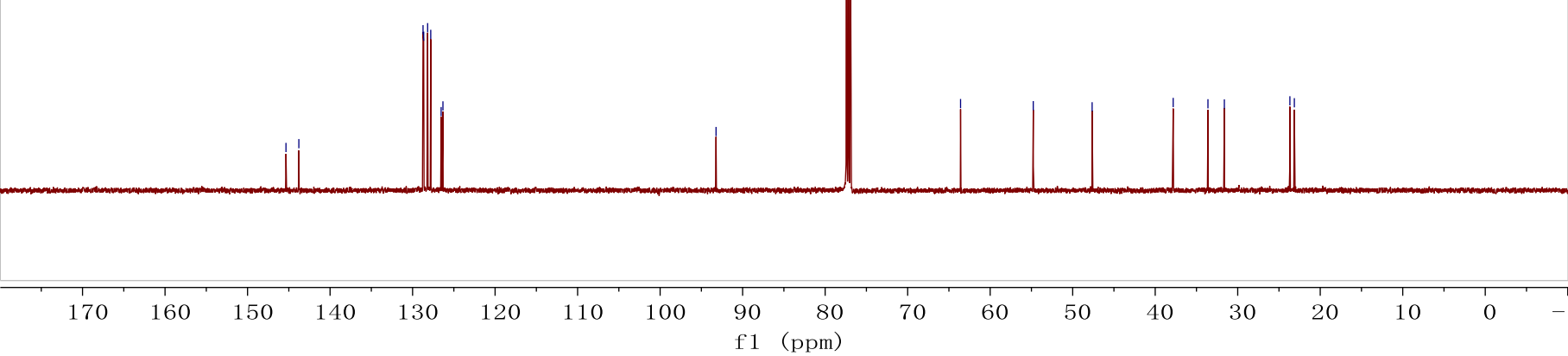


\section{${ }^{1} \mathrm{H}$ NMR (400 MHz, $\left.\mathrm{CDCl}_{3}\right)$}<smiles></smiles>

$3 \mathbf{t}$
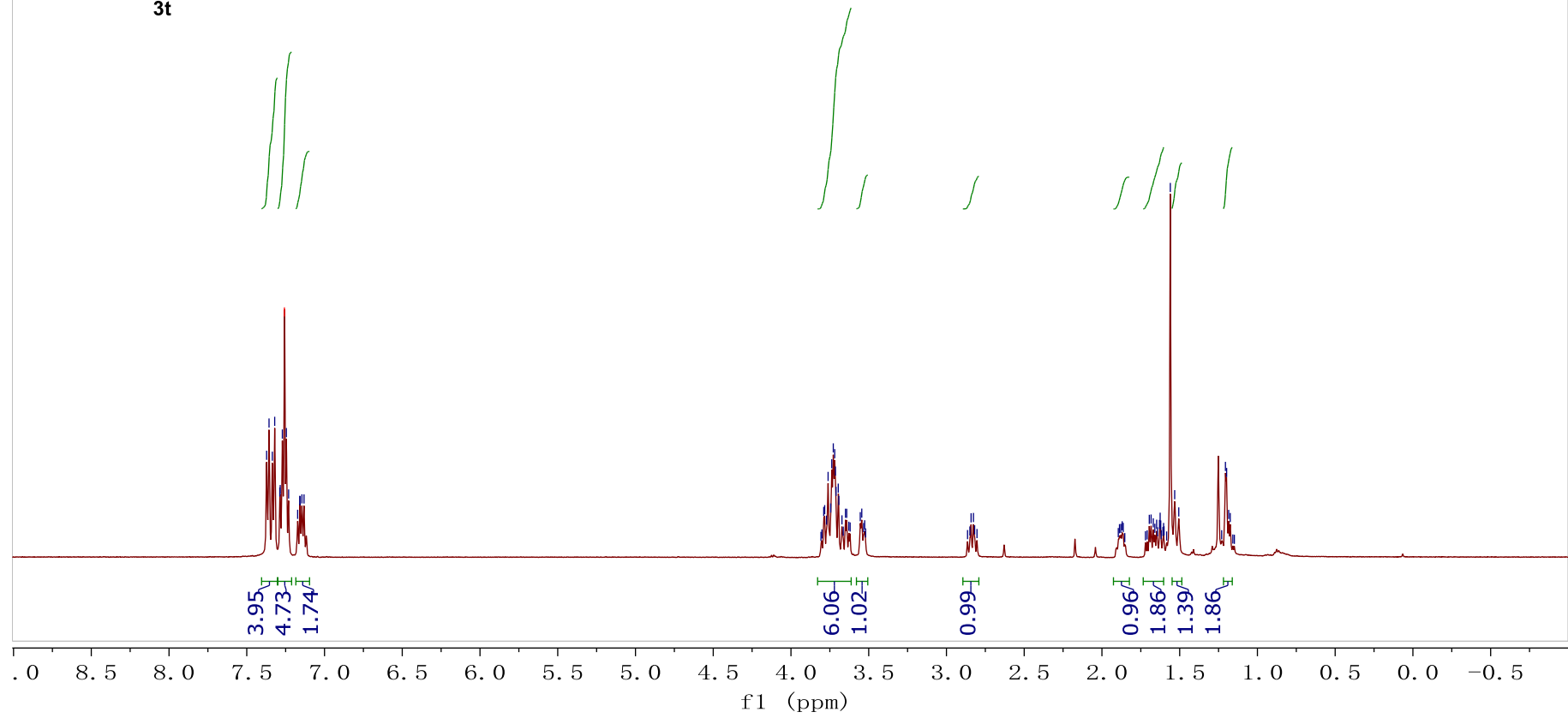

\section{${ }^{13} \mathrm{C}$ NMR (100 MHz, $\left.\mathrm{CDCl}_{3}\right)$}

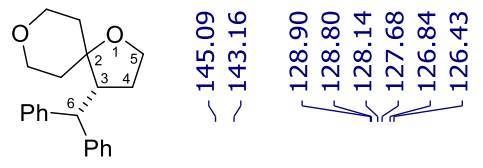

3t

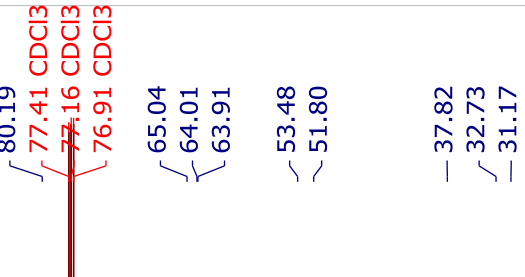
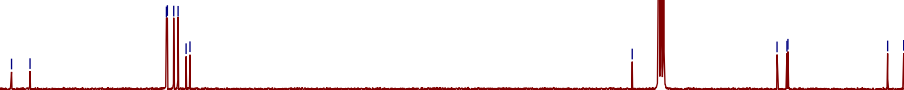


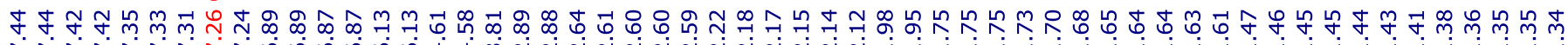

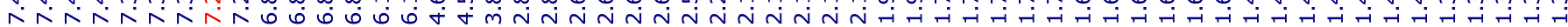

\section{${ }^{1} \mathrm{H}$ NMR (400 MHz, $\left.\mathrm{CDCl}_{3}\right)$}

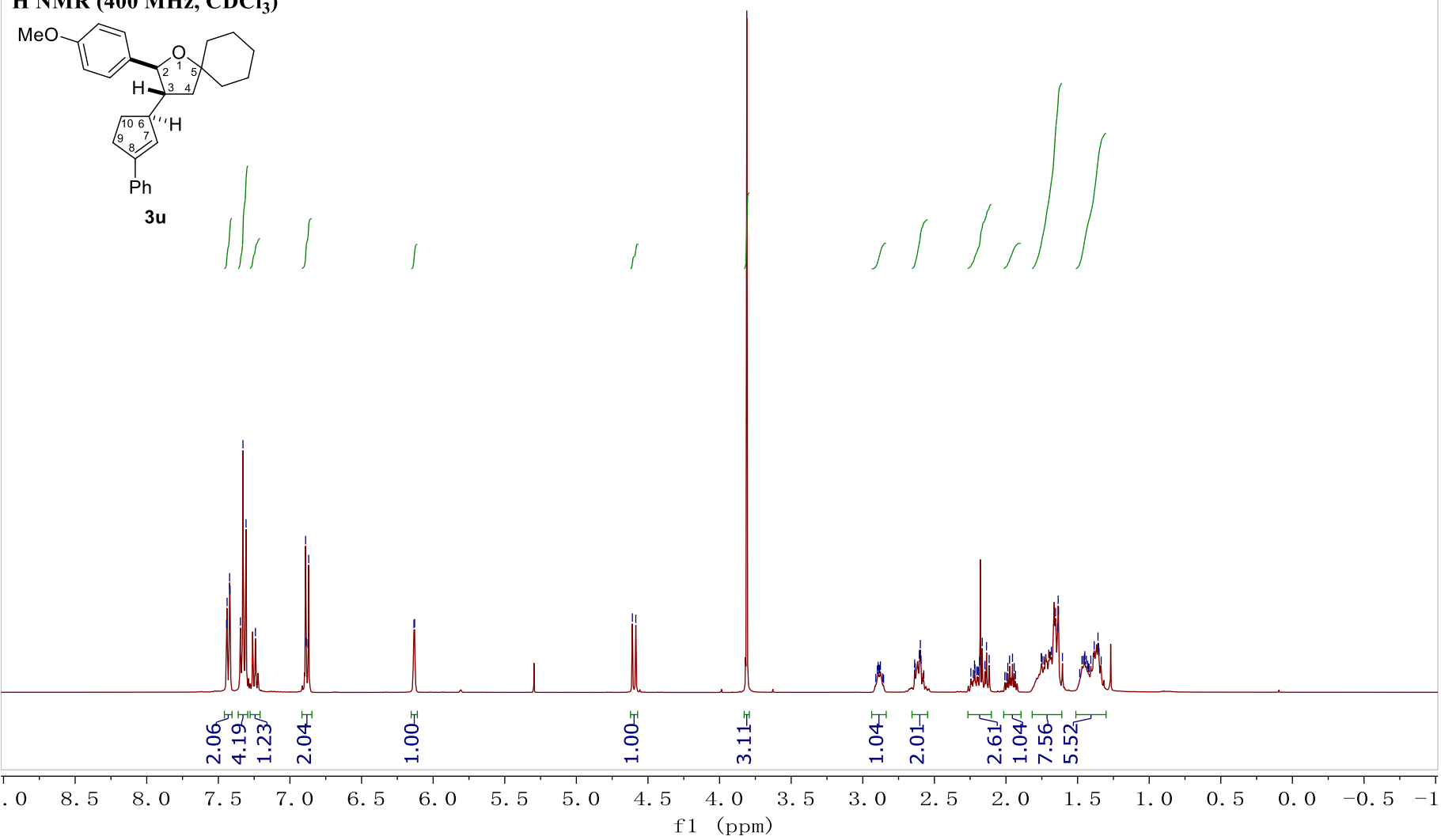

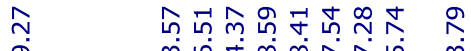

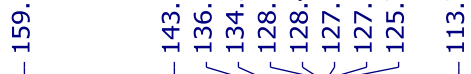

${ }^{13} \mathrm{C}$ NMR (100 MHz, $\left.\mathrm{CDCl}_{3}\right)$

$\mathrm{MeO}$

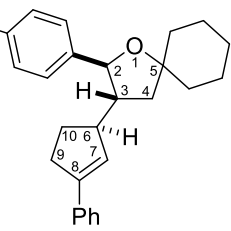

3u

\section{元产}

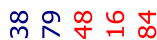

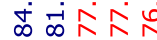

$>>1$

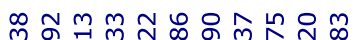

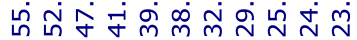

r

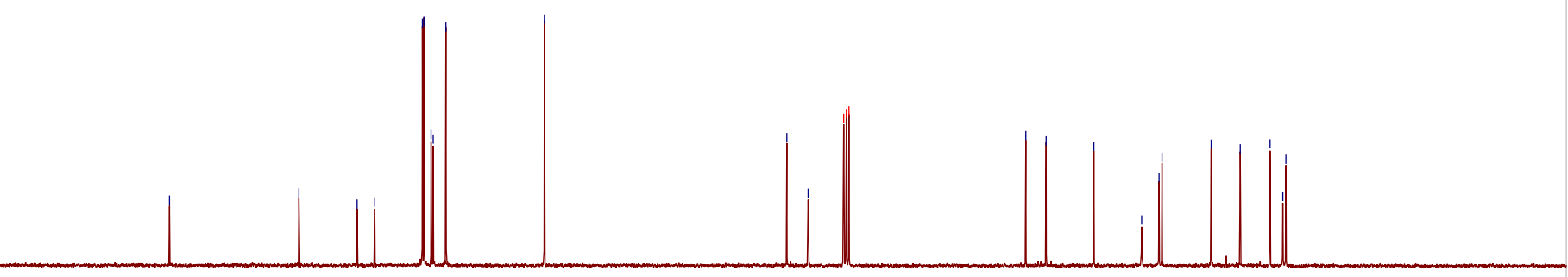

170

$\begin{array}{llll}160 & 150 & 140 & 130\end{array}$

120

110

100 
嘀

J 궀

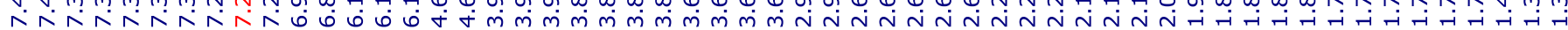
${ }_{1}^{1} \mathrm{NMR}\left(400 \mathrm{MHz}, \mathrm{CDCC}_{3}\right)$
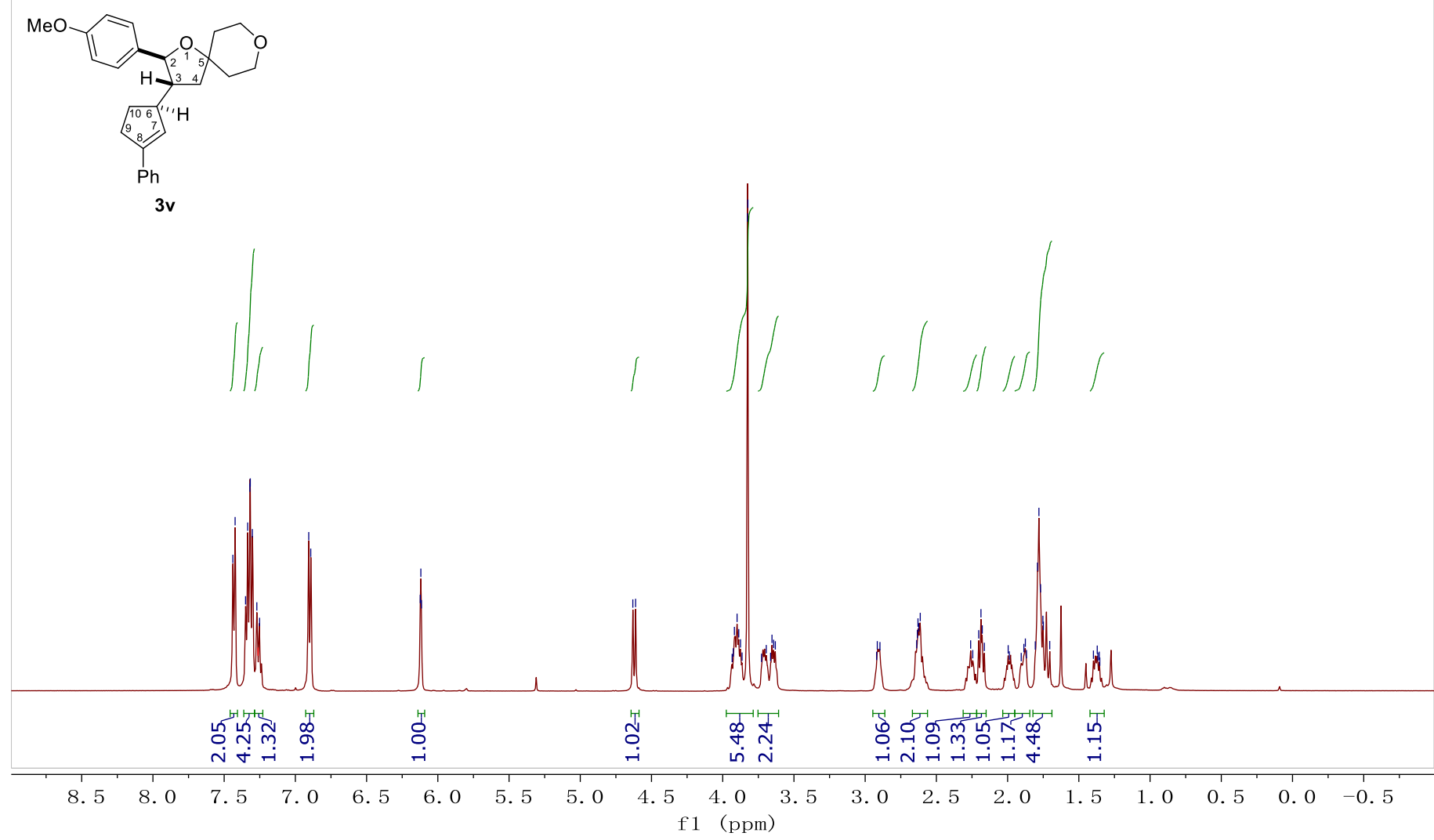

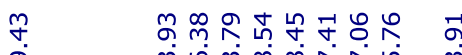

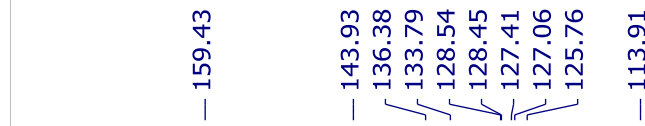

产 $\frac{m}{u} \frac{m}{u}$

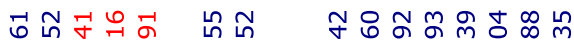

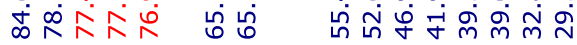

\section{${ }^{13} \mathrm{C}$ NMR (100 MHz, $\left.\mathrm{CDCl}_{3}\right)$}

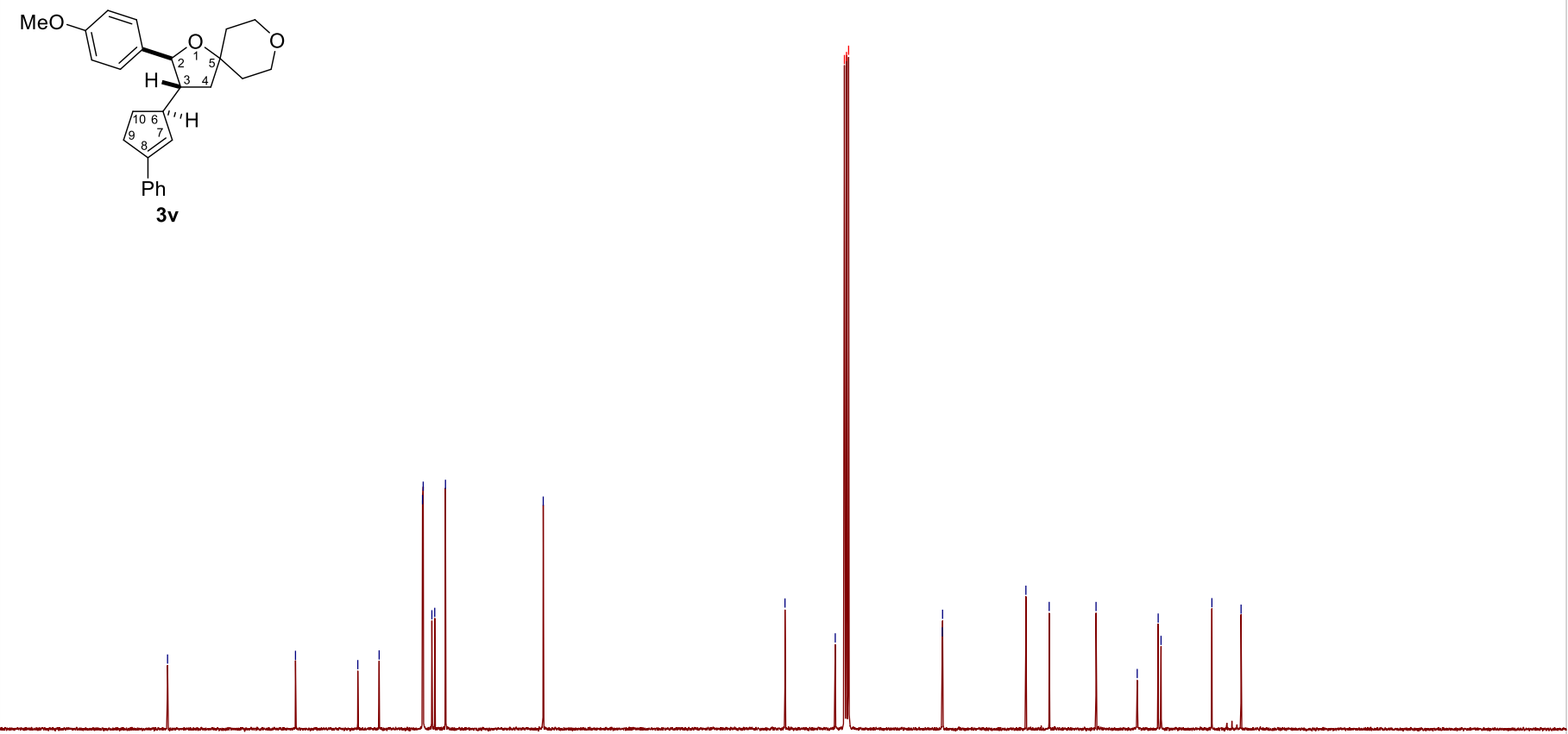


mై

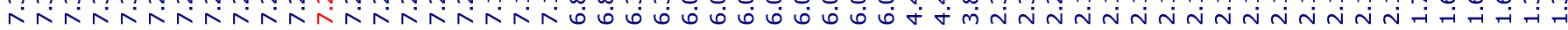

${ }_{1}{ }_{\left.\mathrm{HMR}(400 \mathrm{MHz}, \mathrm{CDC})_{3}\right)}$

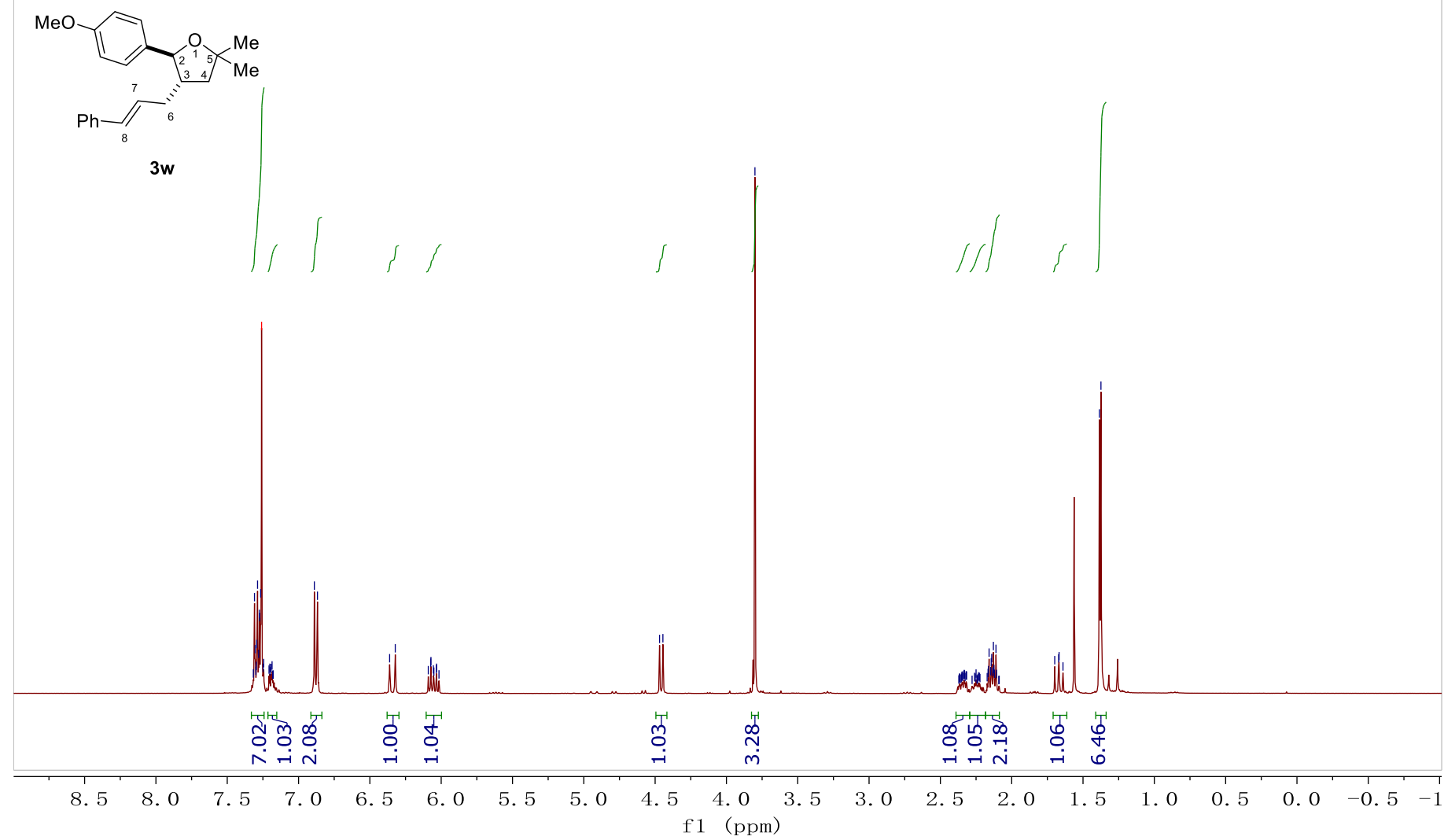

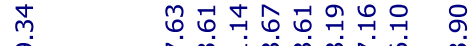

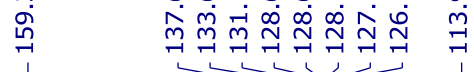

${ }^{13} \mathrm{C}$ NMR (100 MHz, $\mathrm{CDCl}_{3}$ )

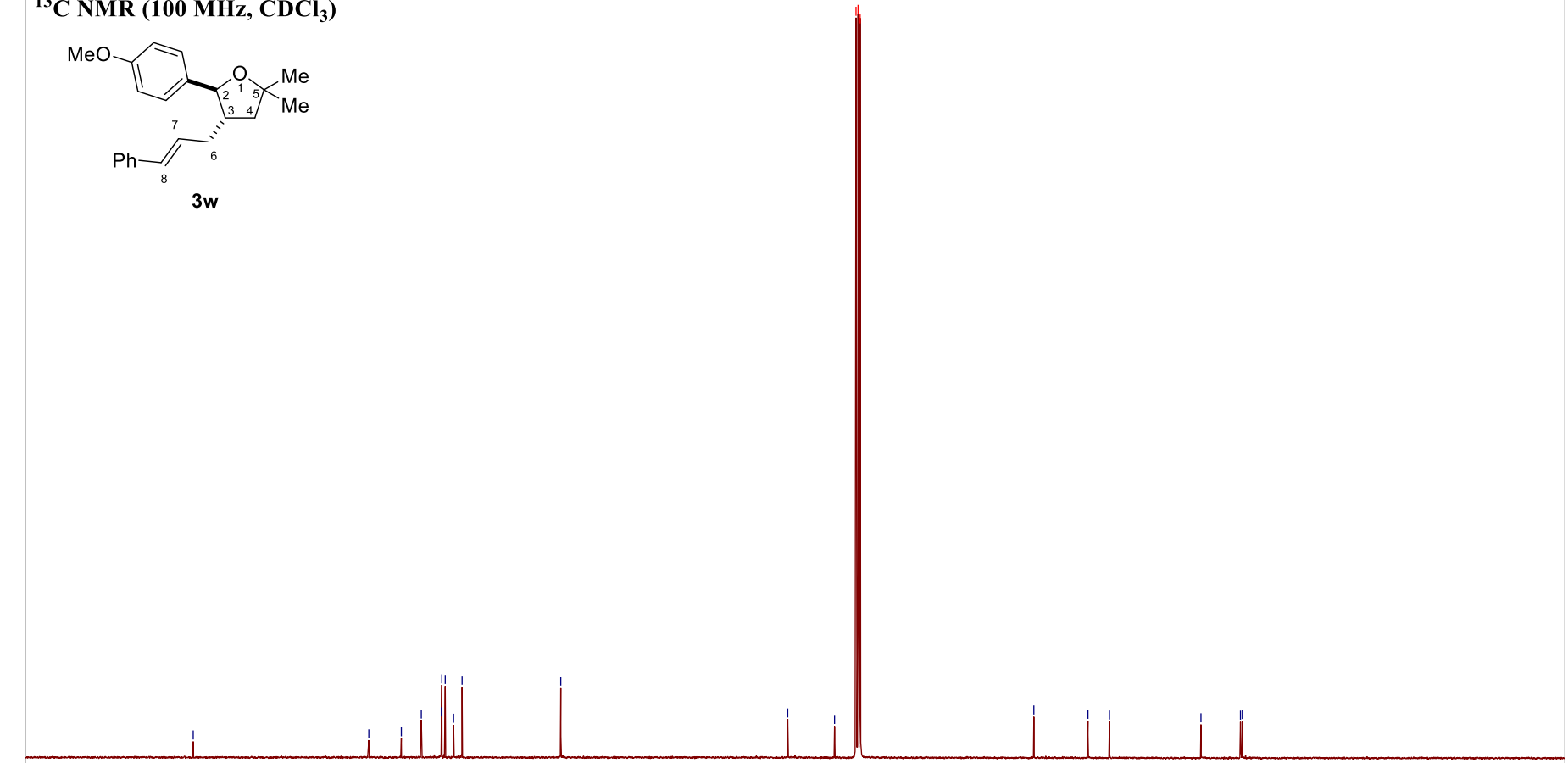

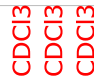

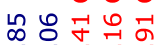
岗官卡官

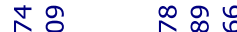

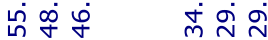

\begin{tabular}{|c|c|c|c|c|c|c|c|c|c|c|c|c|c|c|c|c|c|c|}
\hline 80 & 170 & 160 & 150 & 140 & 130 & 120 & 110 & 100 & 90 & 80 & 70 & 60 & 50 & 40 & 30 & 20 & 10 & $\begin{array}{l}1 \\
0\end{array}$ \\
\hline
\end{tabular}




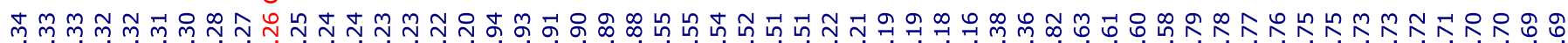

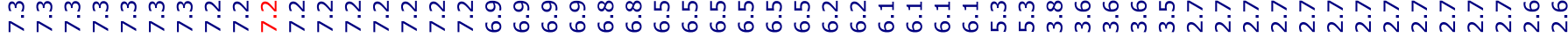

\section{${ }^{1} \mathrm{H}$ NMR (500 MHz, $\mathrm{CDCl}_{3}$ )}

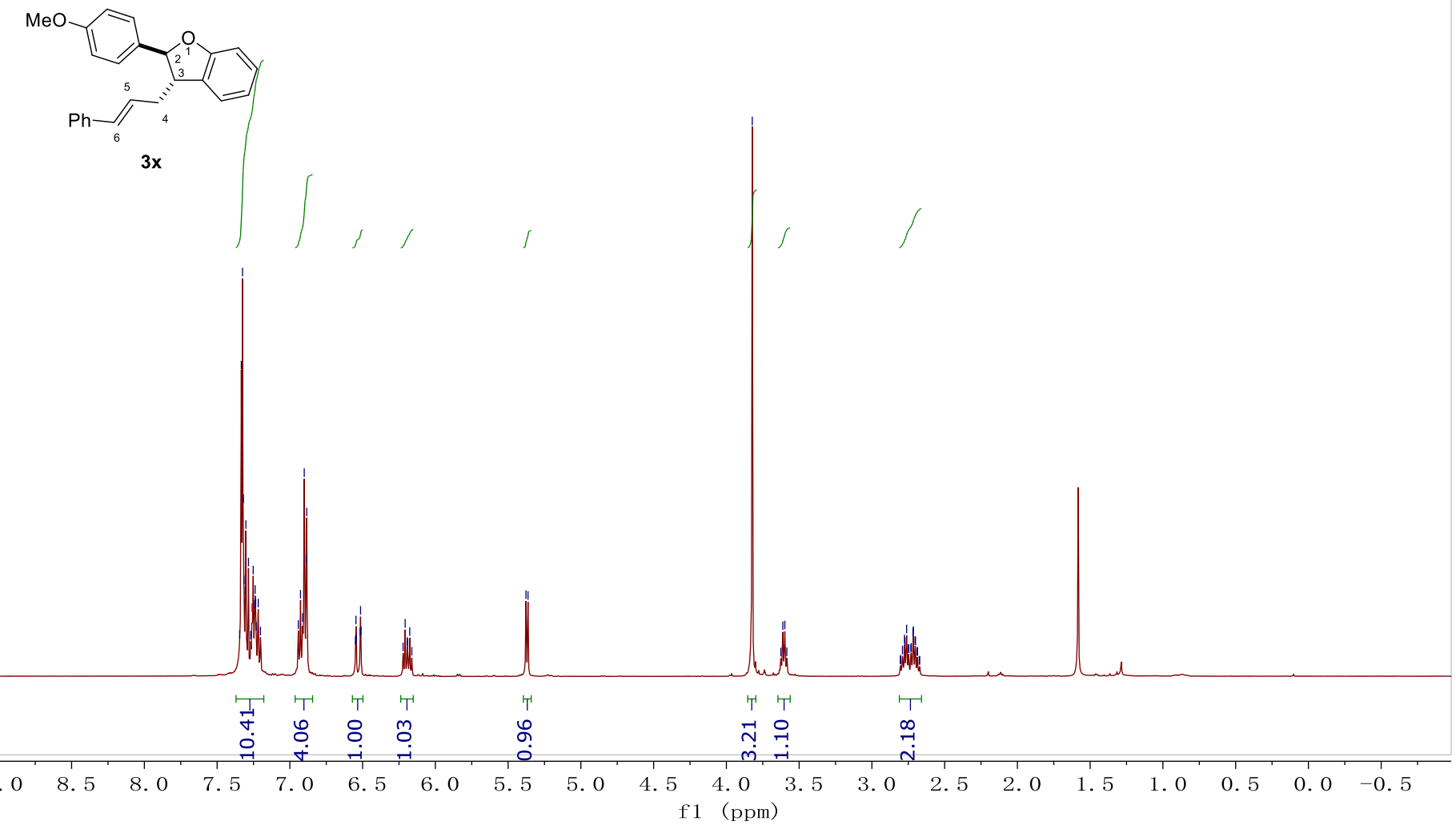

产

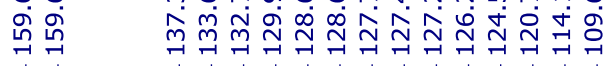

ํㅡㄴ

\section{${ }^{13} \mathrm{C}$ NMR (125 MHz, $\left.\mathrm{CDCl}_{3}\right)$}

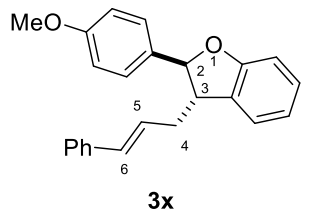

$3 \mathbf{x}$

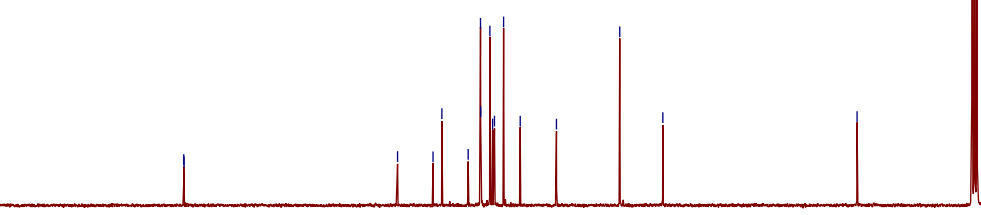


${ }^{1} \mathrm{H}-{ }^{1} \mathrm{H}$ COSY - $90\left(500 \mathrm{MHz}, \mathrm{CDCl}_{3}\right)$

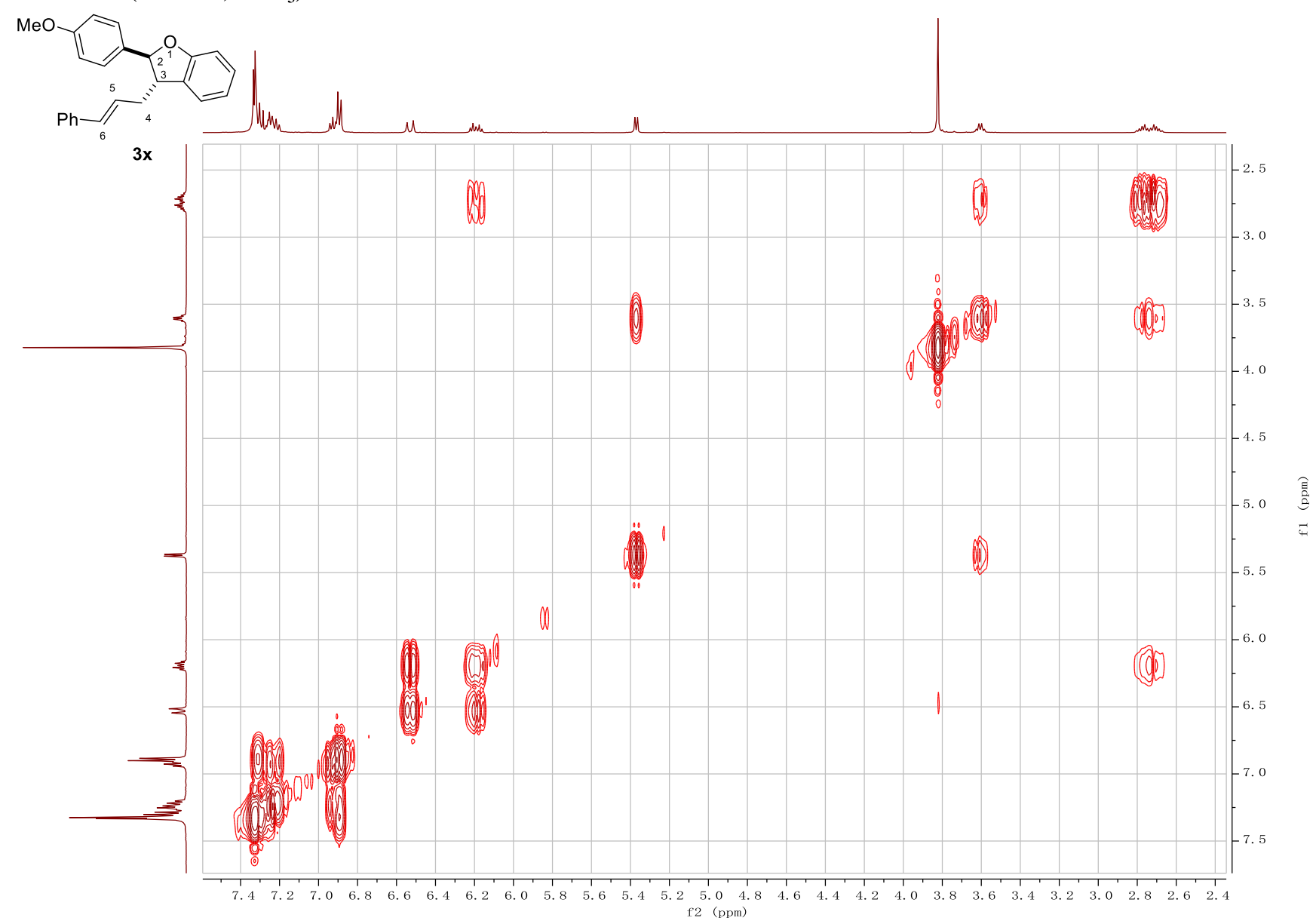

${ }^{1} \mathrm{H}(500 \mathrm{MHz})-{ }^{13} \mathrm{C}(125 \mathrm{MHz}) \mathrm{HSQC}\left(\mathrm{CDCl}_{3}\right)$

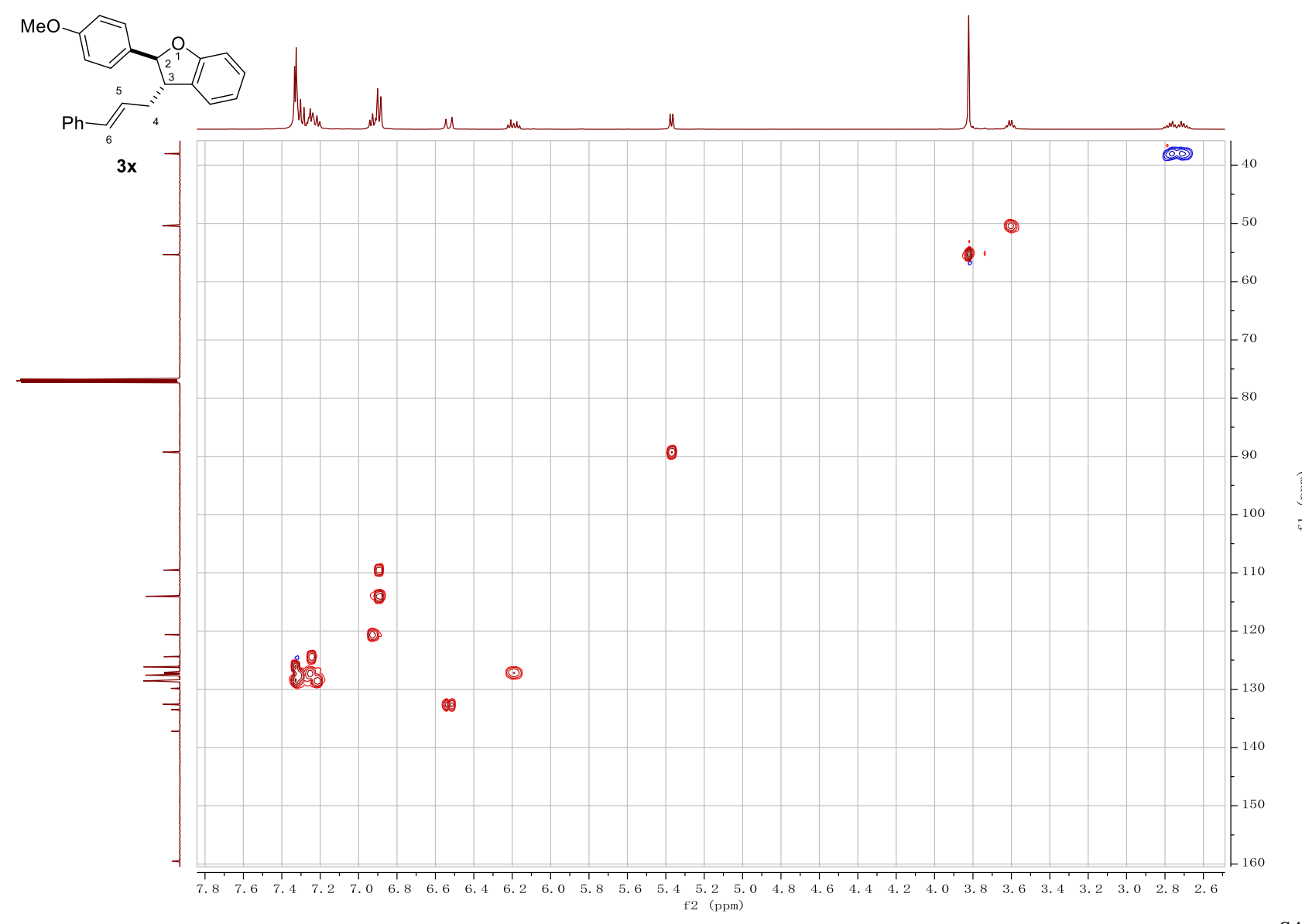




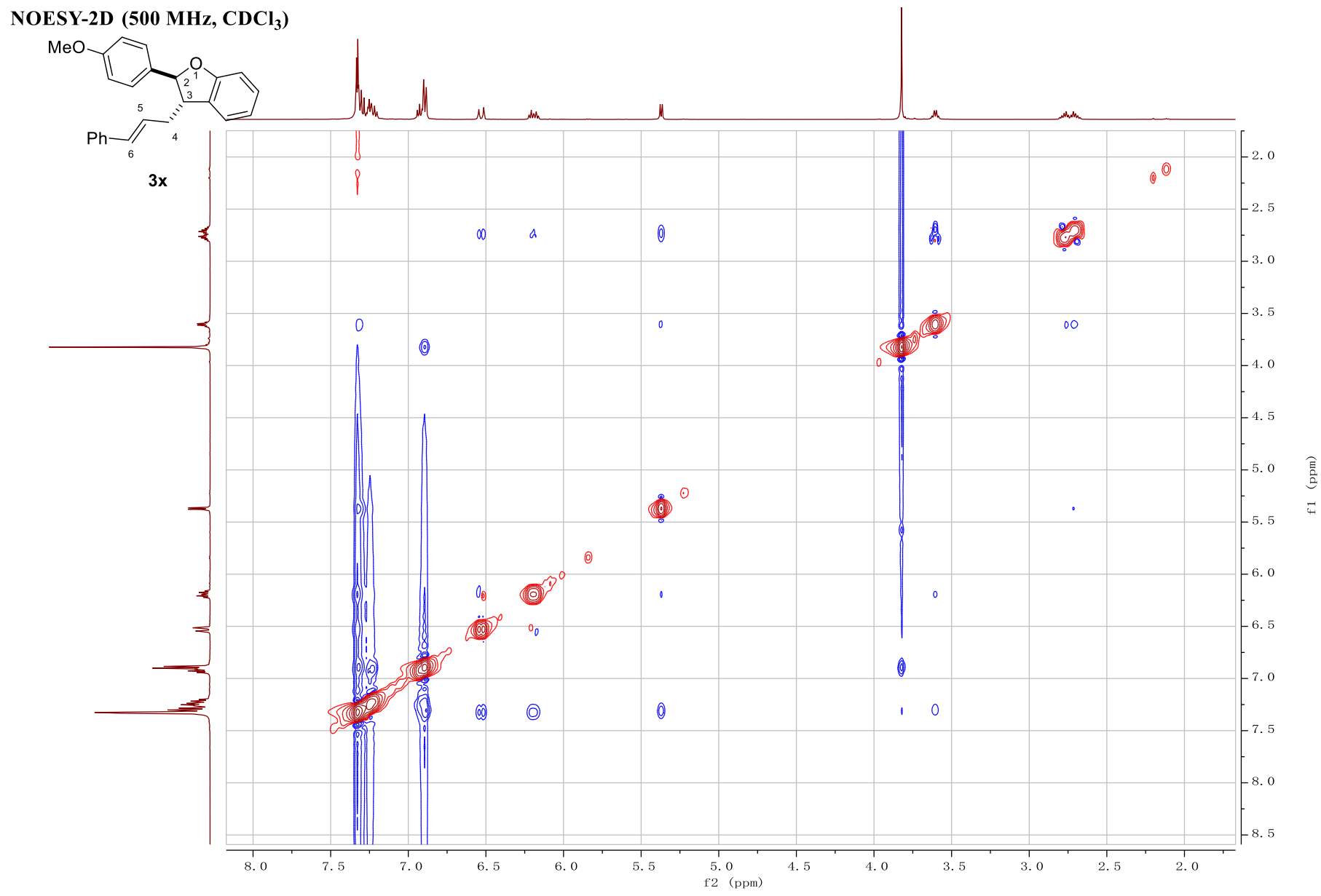

S49 
${ }^{1} \mathrm{H}$ NMR (400 MHz, $\left.\mathrm{CDCl}_{3}\right)$

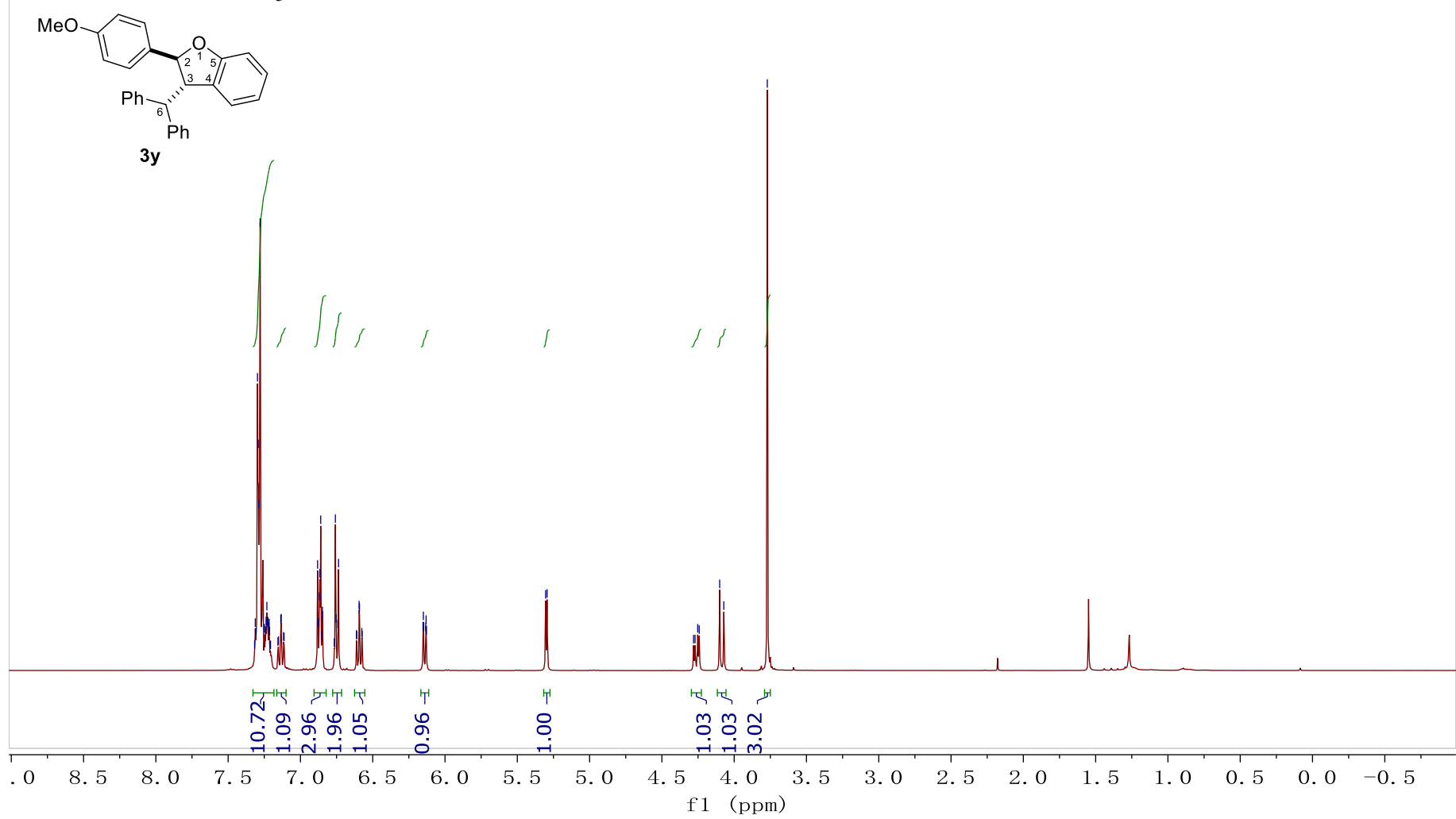

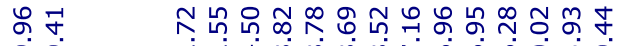

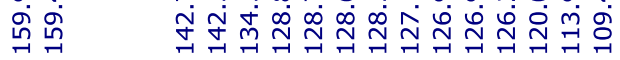

ํㅡㄹ

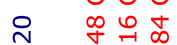

1

\section{${ }^{13} \mathrm{C}$ NMR (100 MHz, $\left.\mathrm{CDCl}_{3}\right)$}

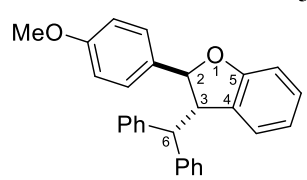

3y

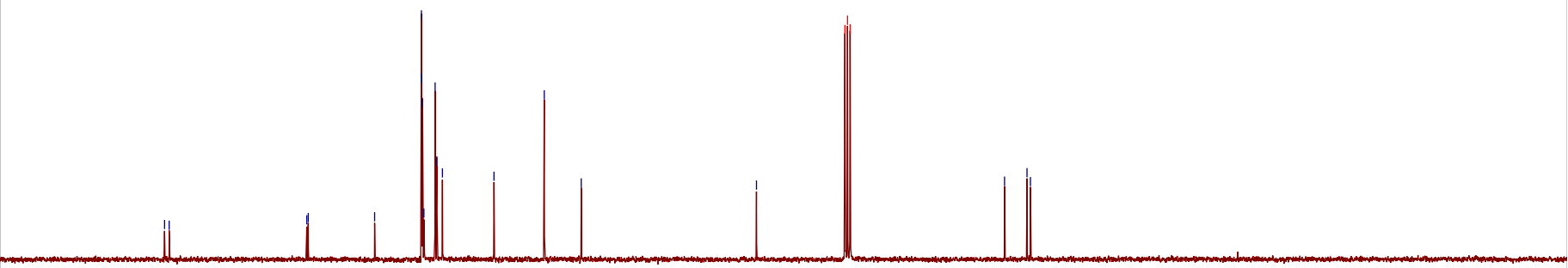


峁

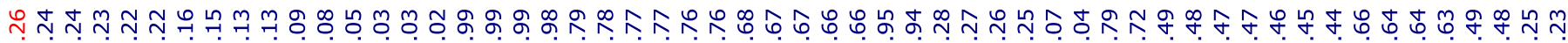

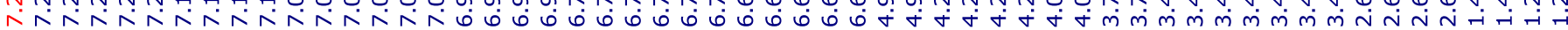

${ }^{1} \mathrm{H}$ NMR $\left(500 \mathrm{MHz}, \mathrm{CDCl}_{3}\right)$<smiles>COc1ccc(-c2oc(C)c(-c3ccccc3)c2-c2ccccc2)cc1</smiles><smiles>COc1ccc(-c2oc(-c3ccccc3)c(-c3ccccc3)c2-c2ccccc2)cc1</smiles>

A

B $\operatorname{dr} 8: 1$ (C3-C4)
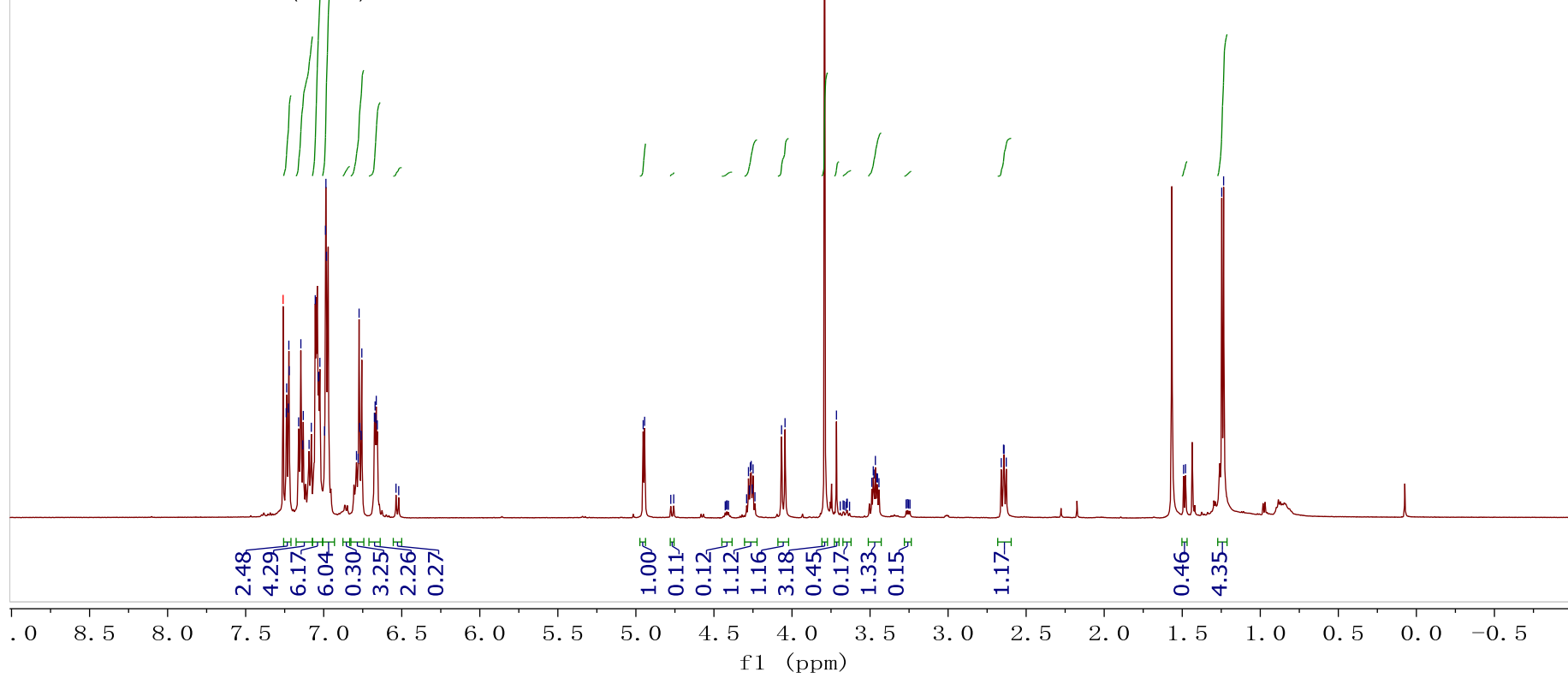

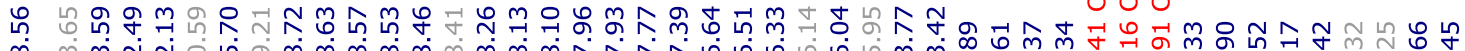

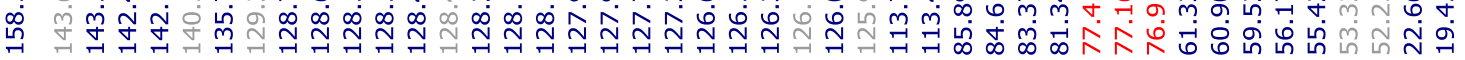
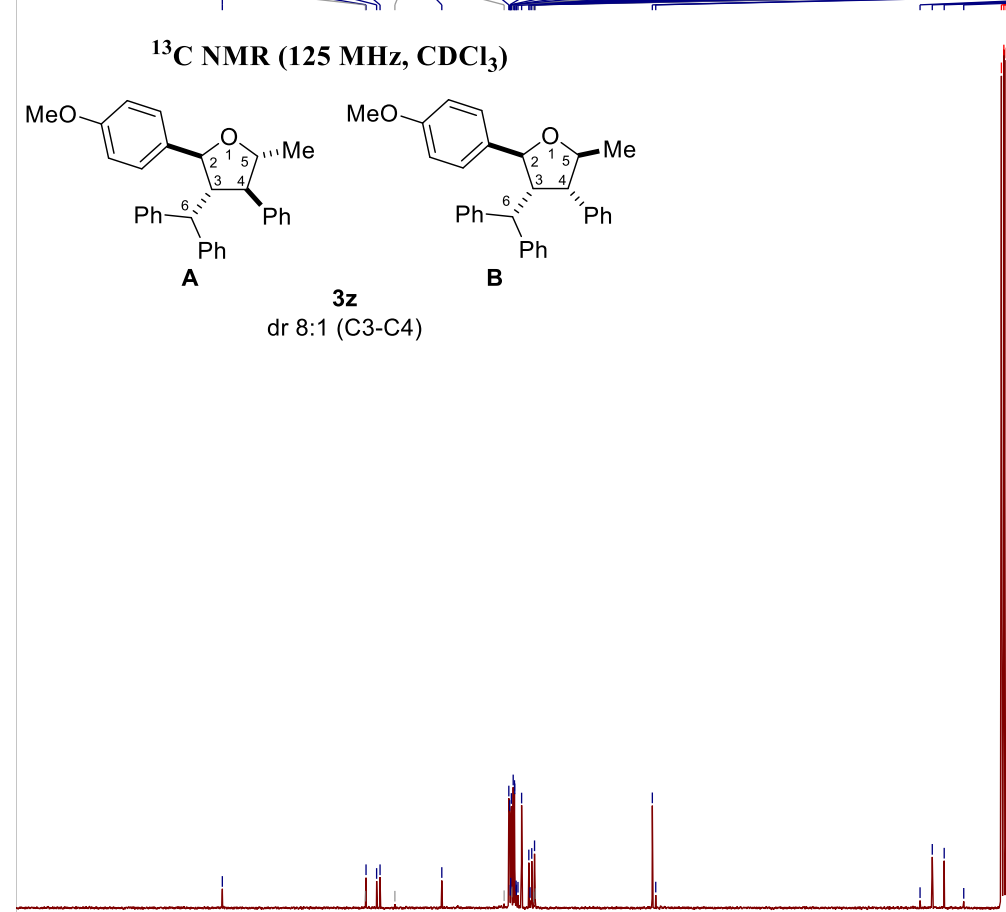

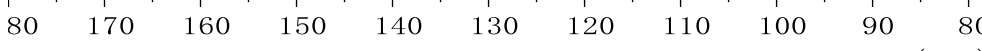


${ }^{1} \mathrm{H}$ - ${ }^{1} \mathrm{H}$ COSY - $90\left(500 \mathrm{MHz}, \mathrm{CDCl}_{3}\right)$
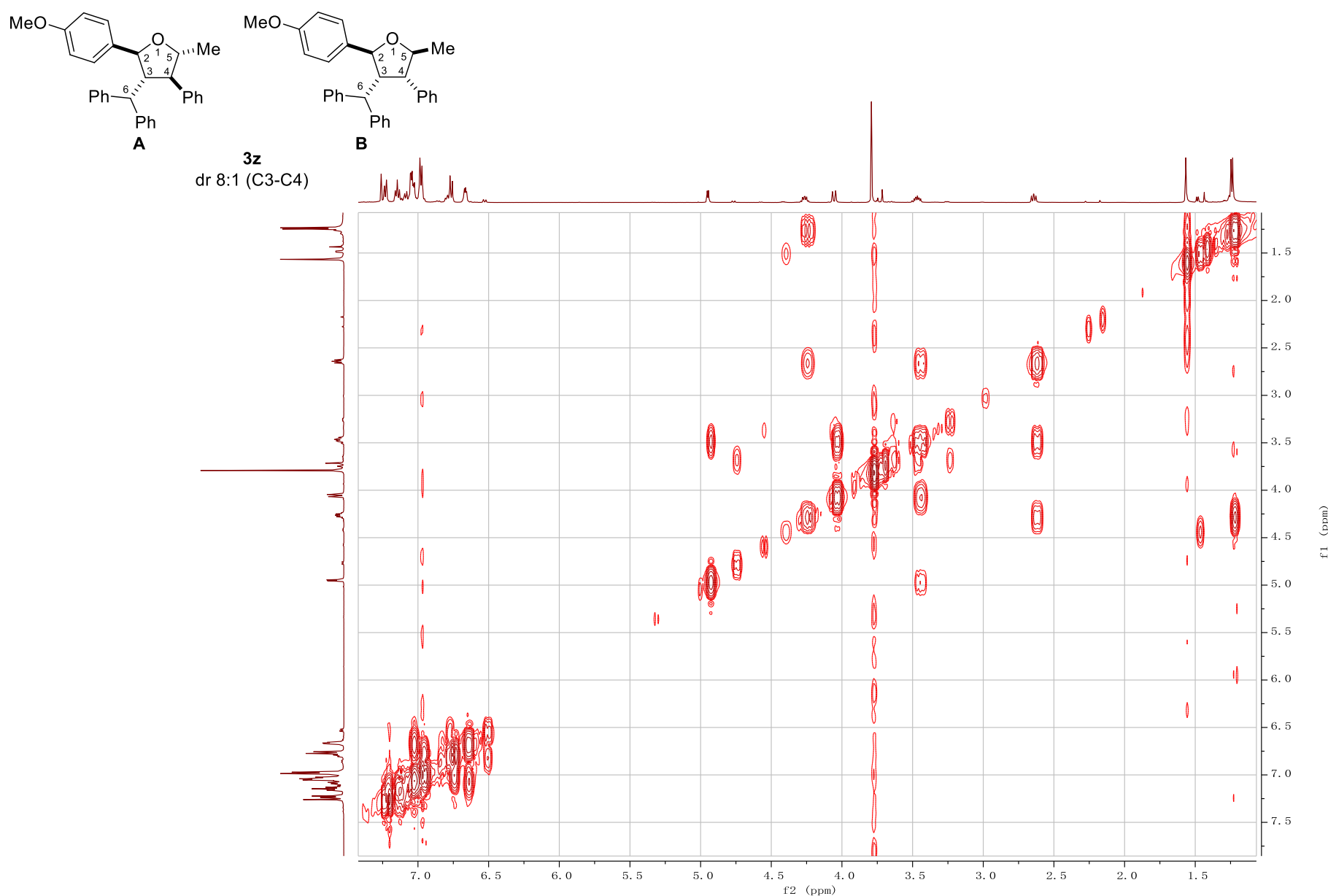

${ }^{1} \mathrm{H}(500 \mathrm{MHz})-{ }^{13} \mathrm{C}(125 \mathrm{MHz}) \mathrm{HSQC}\left(\mathrm{CDCl}_{3}\right)$
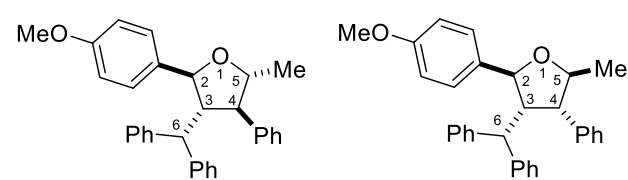

A $^{\mathrm{Ph}}$
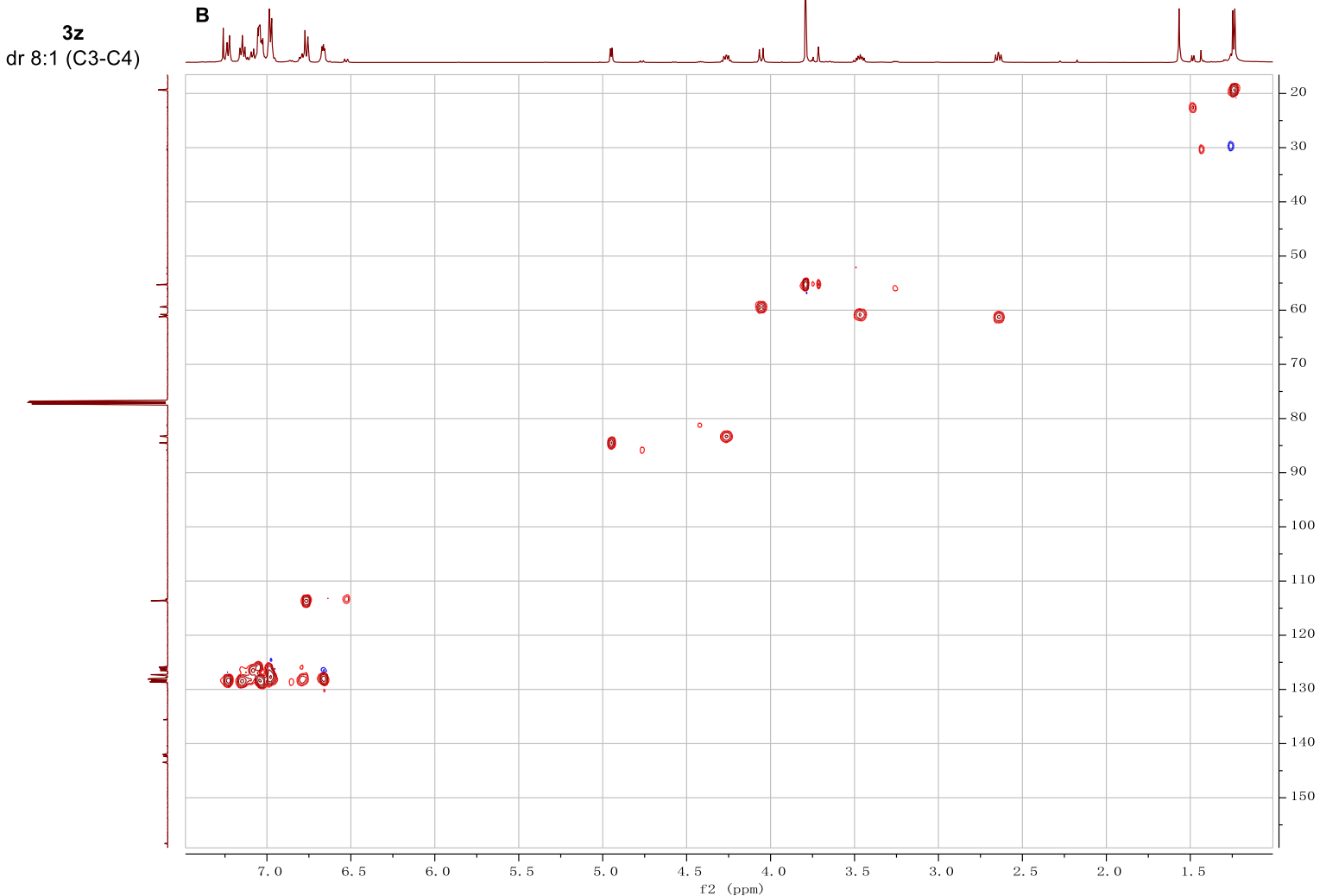


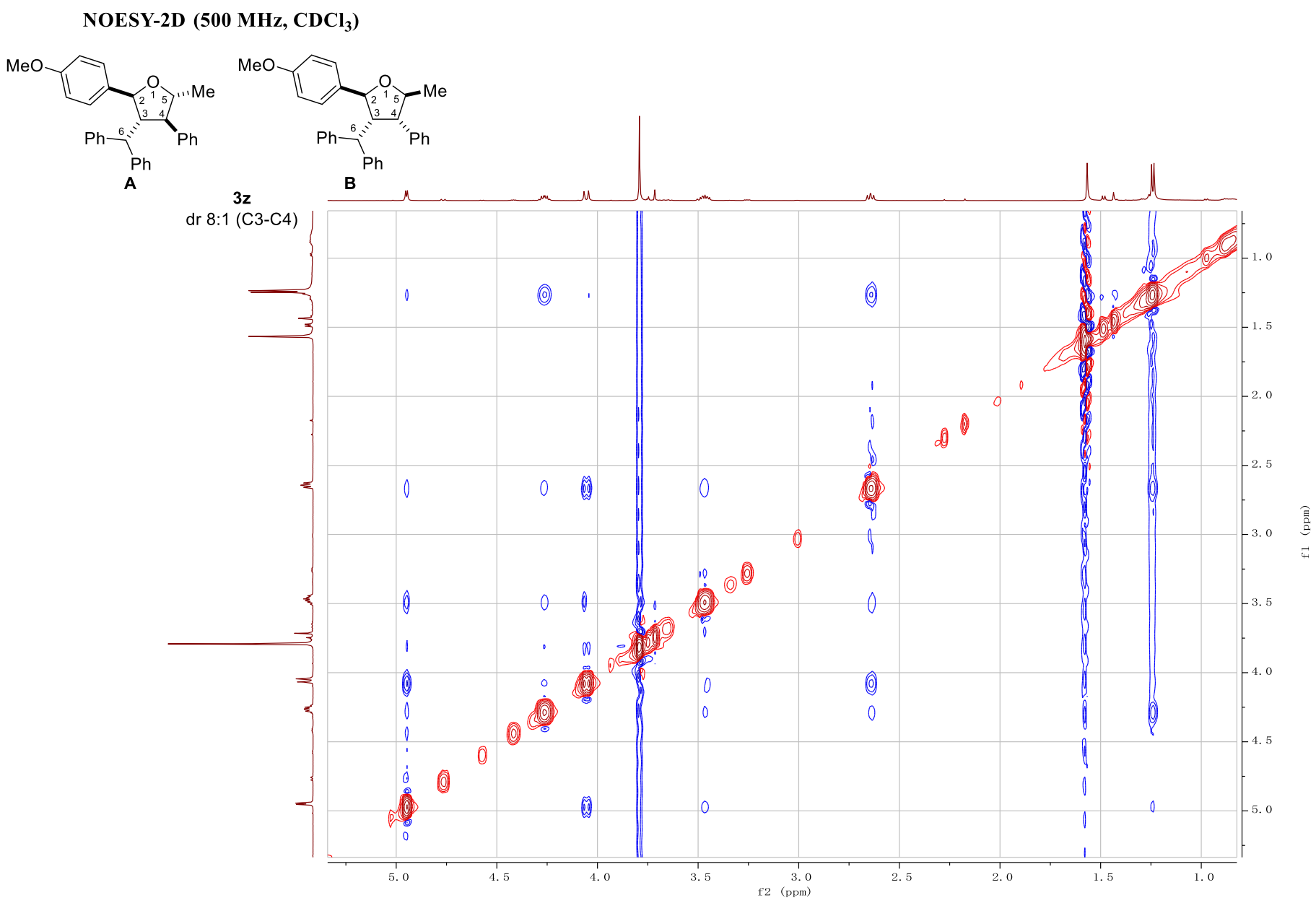

S53 
峁

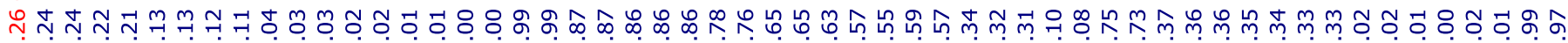

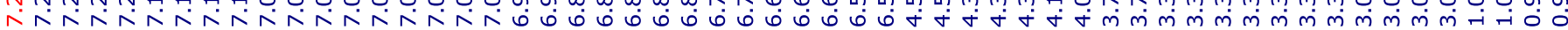

${ }^{1} \mathrm{H}$ NMR (500 MHz, $\left.\mathrm{CDCl}_{3}\right)$

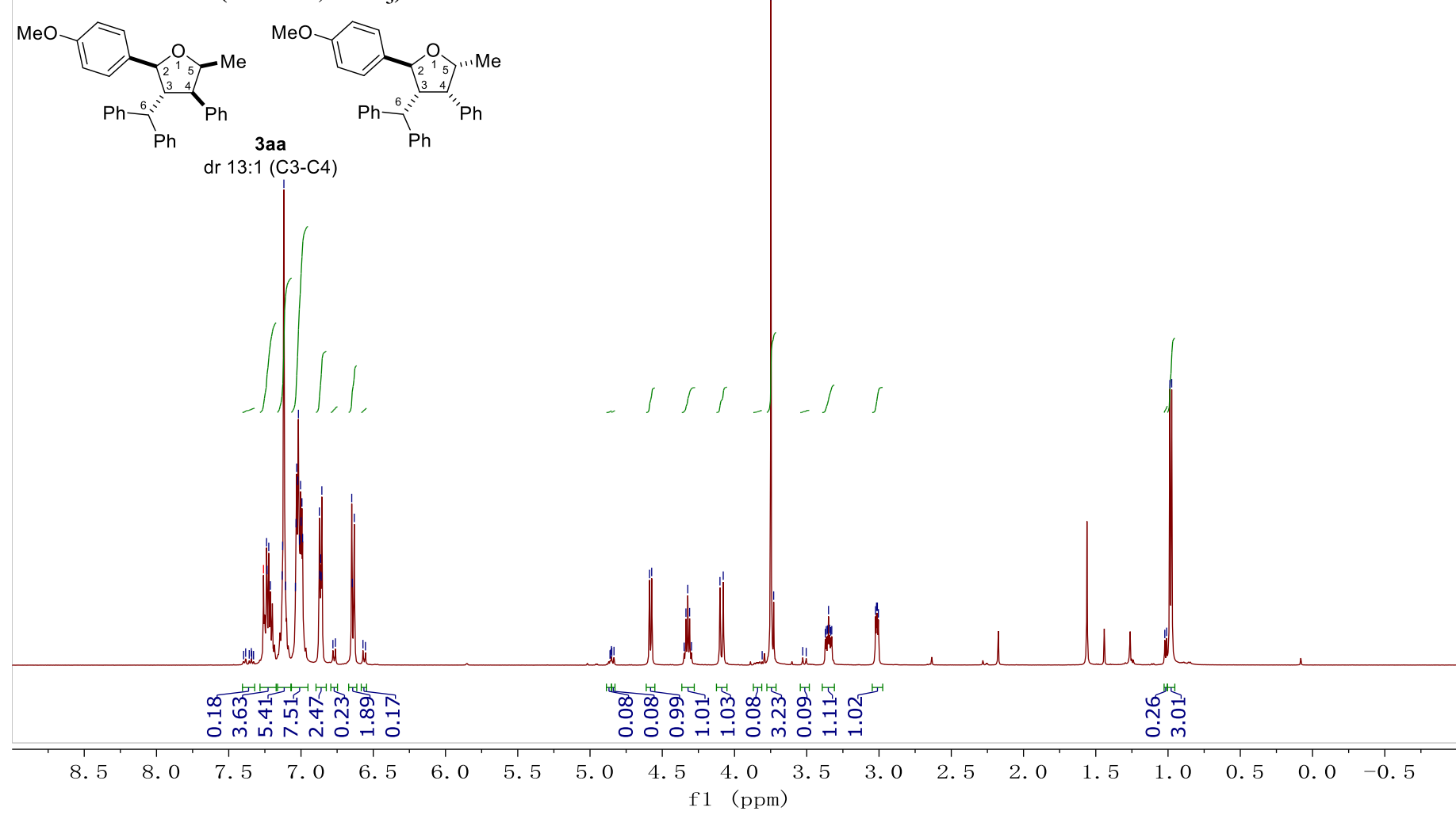

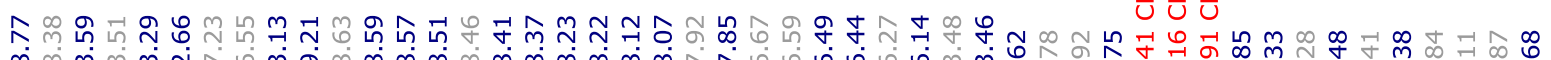

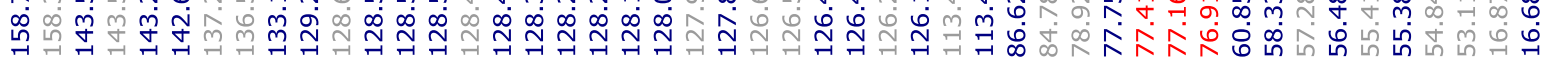

\section{${ }^{13} \mathrm{C}$ NMR (125 MHz, $\left.\mathrm{CDCl}_{3}\right)$}

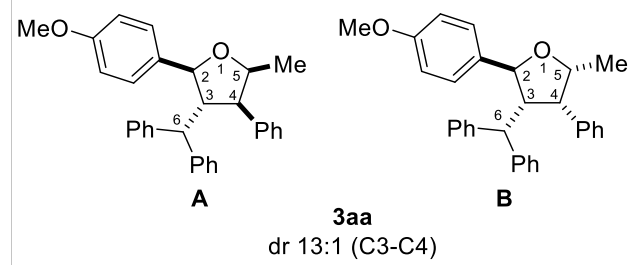

dr 13:1 (C3-C4)

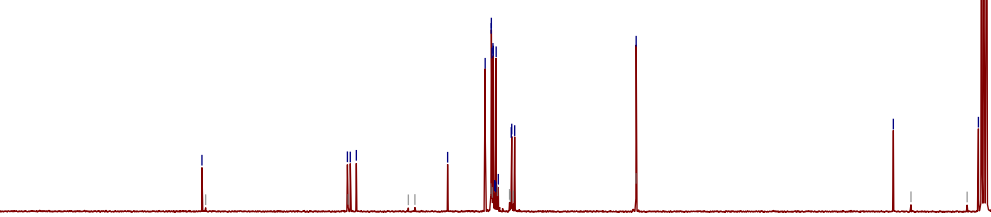

$80 \quad 170 \quad 160 \quad 150 \quad 140 \quad 130 \quad 120 \quad 110 \quad 100 \quad 90 \quad 80$ 

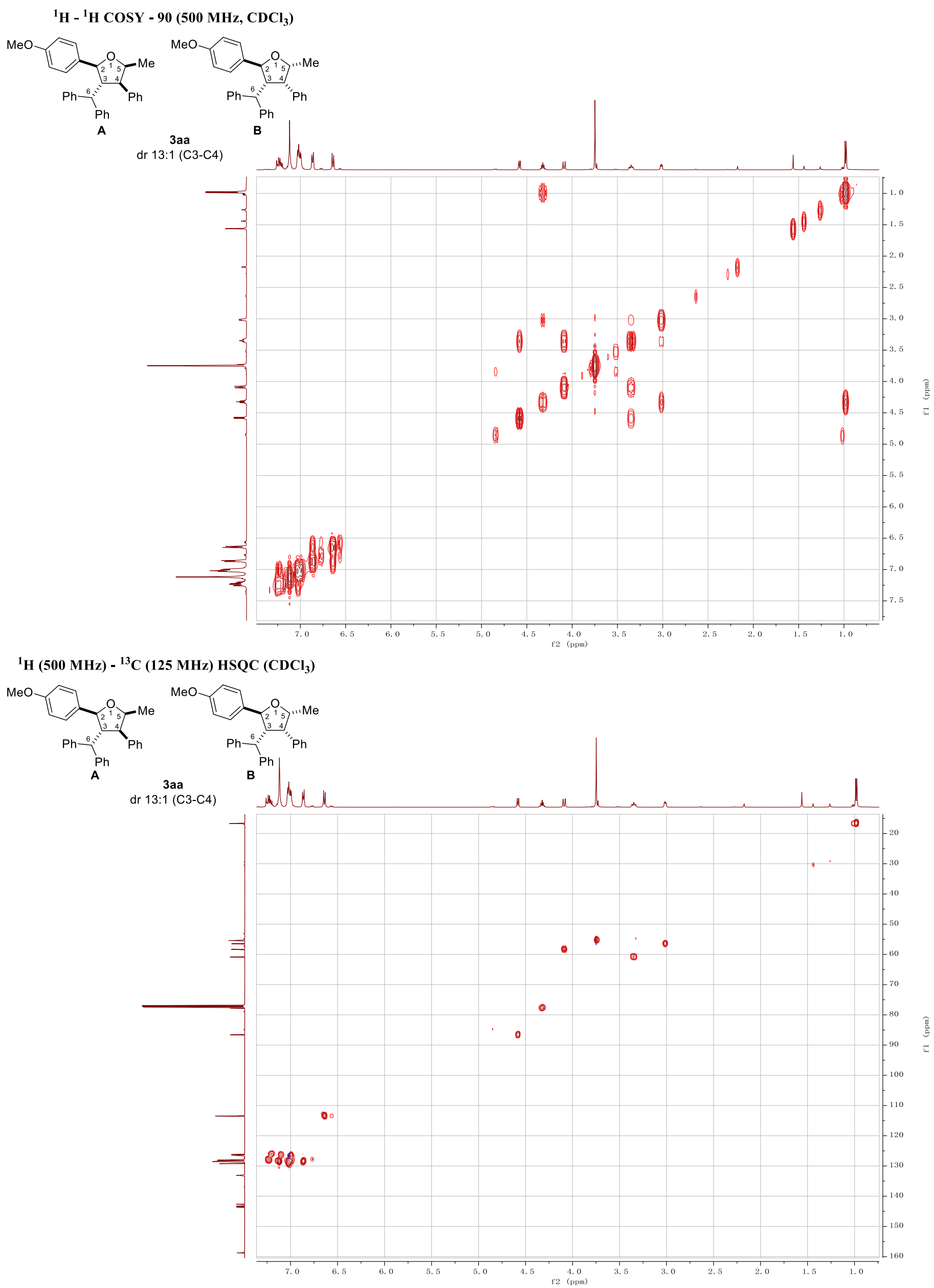

S55 
NOESY-2D (500 MHz, CDCl $\mathrm{CD}_{3}$

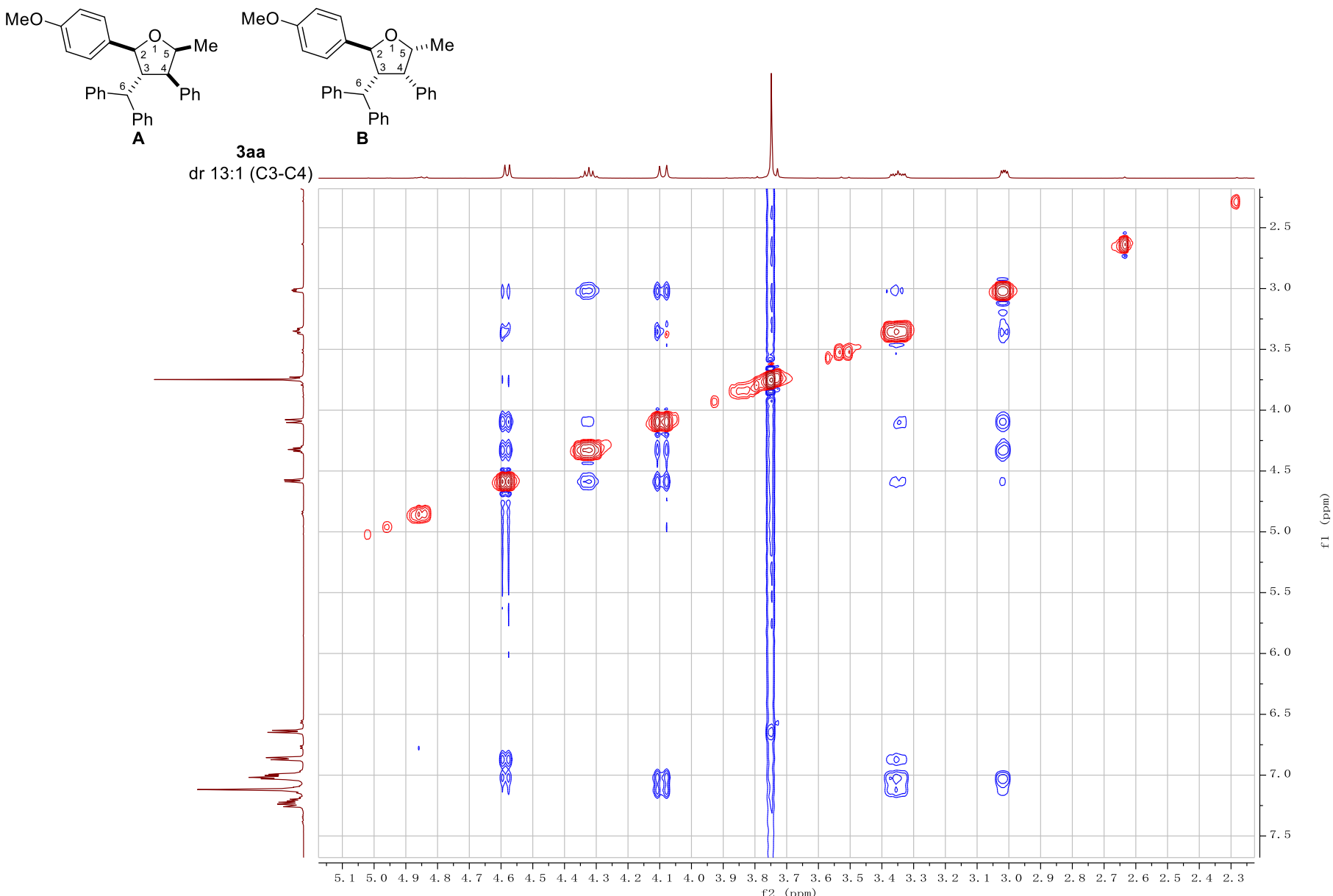




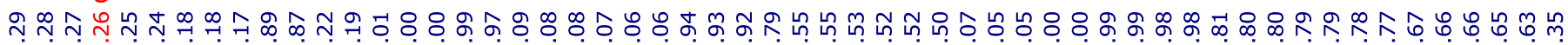
ヘ ヘ

${ }^{1} \mathrm{H}$ NMR (400 MHz, $\left.\mathrm{CDCl}_{3}\right)$
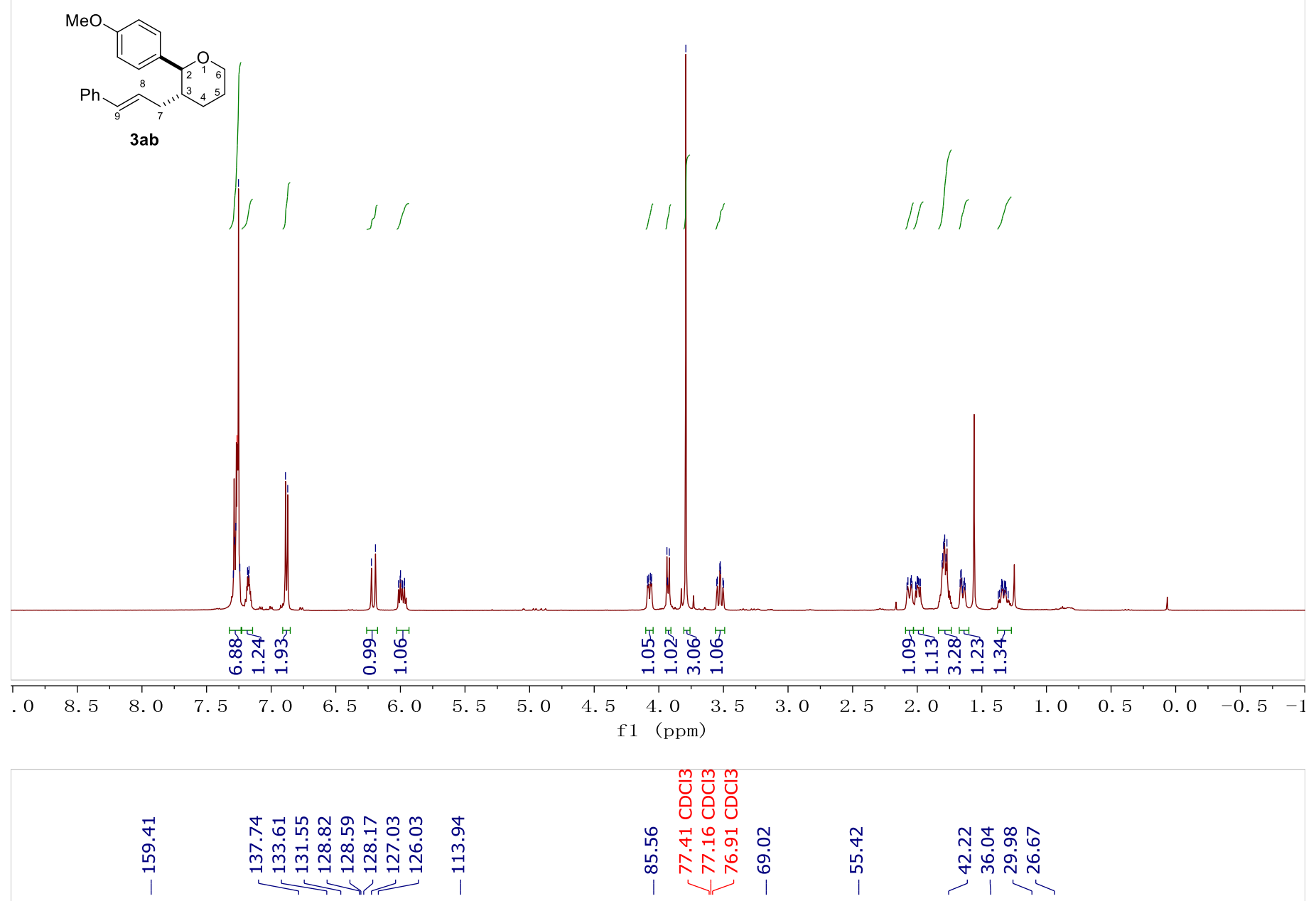

${ }^{13} \mathrm{C}$ NMR (100 $\mathrm{MHz}, \mathrm{CDCl}_{3}$ )
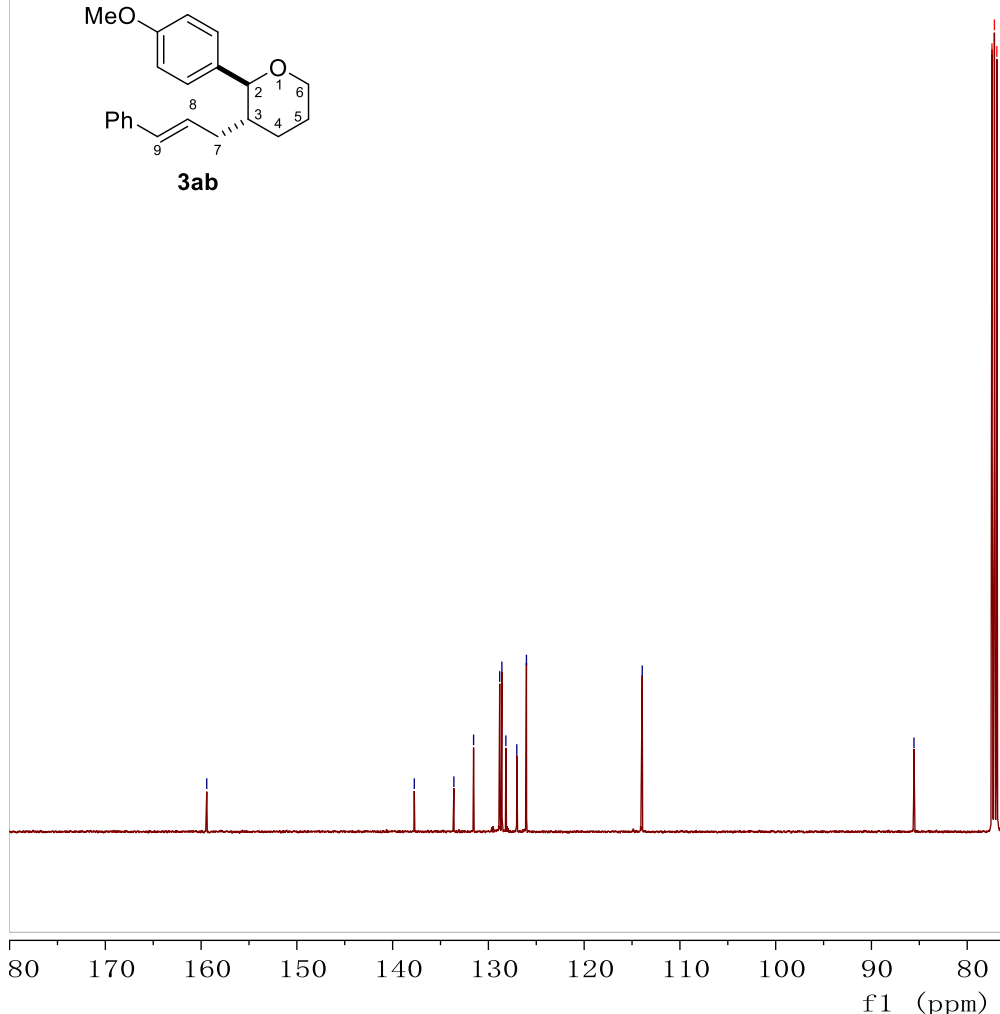
${ }^{1} \mathrm{H}$ NMR (400 MHz, $\mathrm{CDCl}_{3}$ )

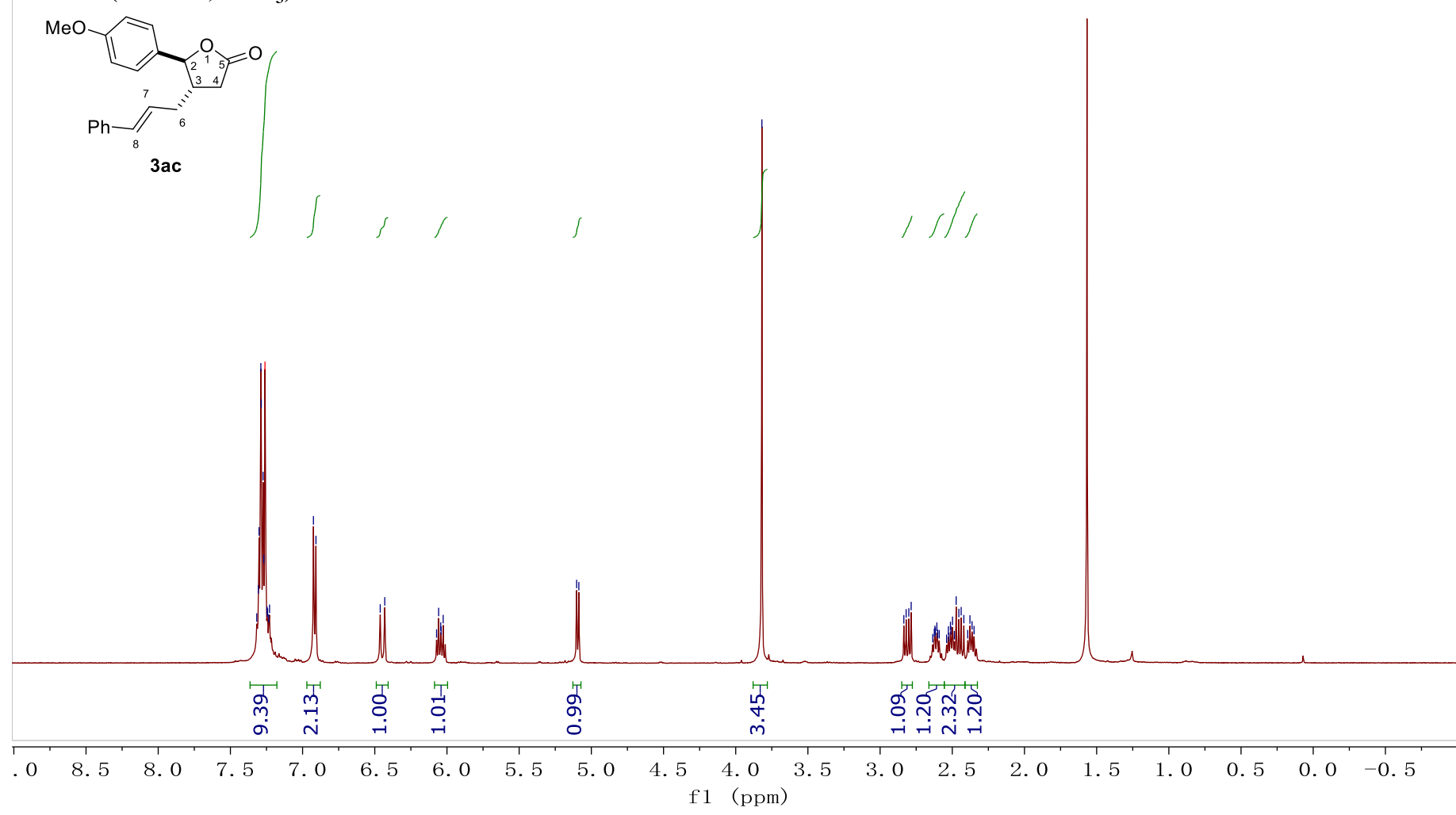

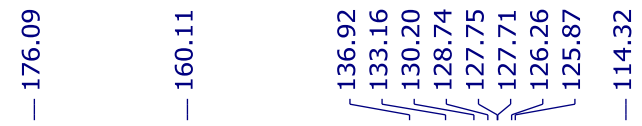

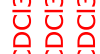

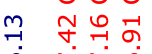

め

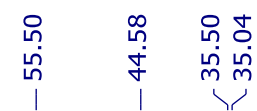

\section{${ }^{13} \mathrm{C}$ NMR (100 MHz, $\left.\mathrm{CDCl}_{3}\right)$}
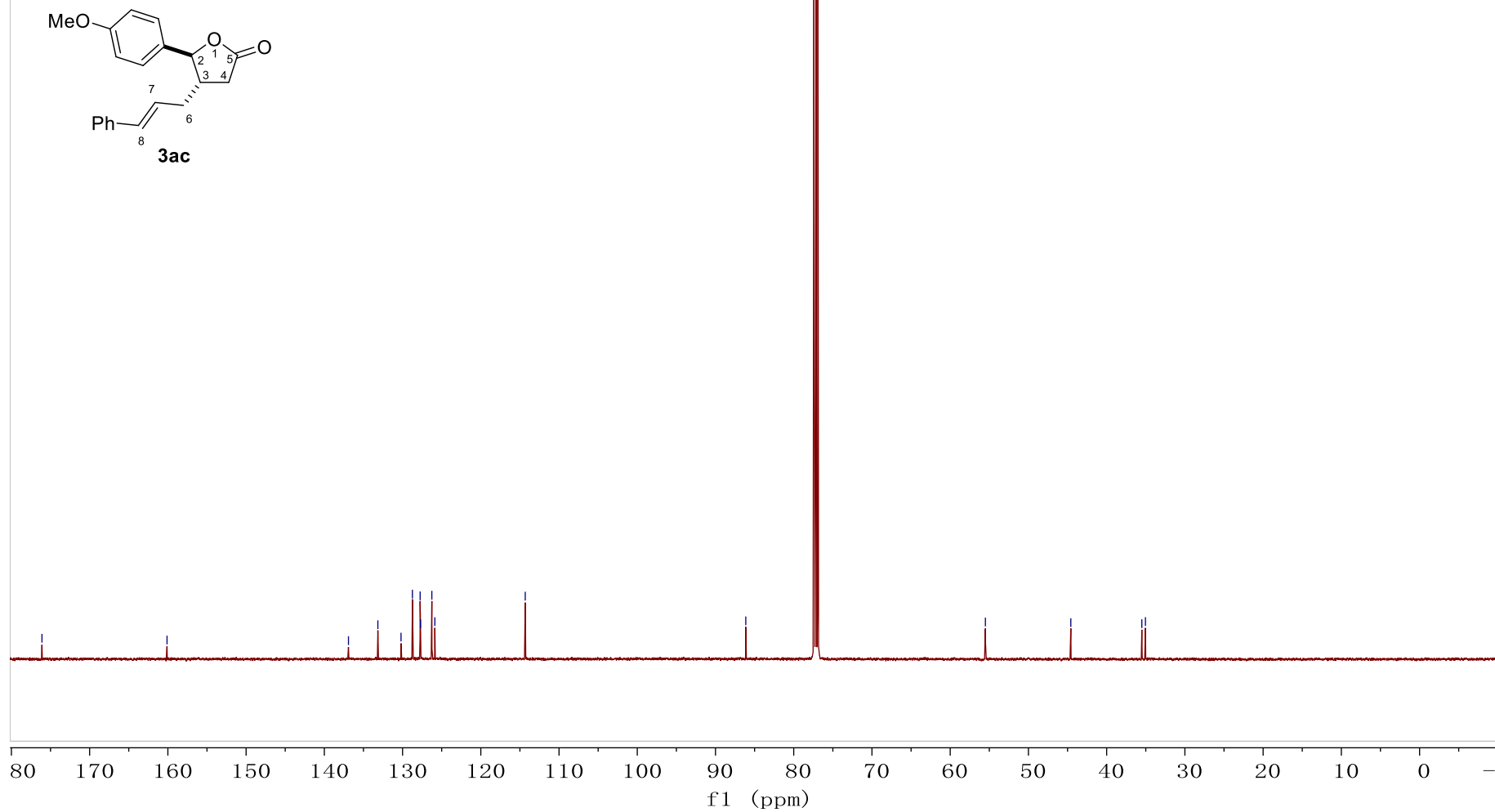
${ }^{1} \mathrm{H}$ NMR (500 MHz, $\left.\mathrm{CDCl}_{3}\right)$

$\mathrm{MeO}$

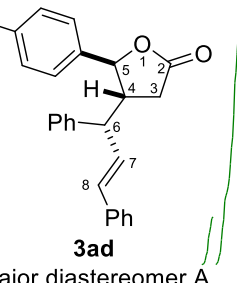

major diastereomer A
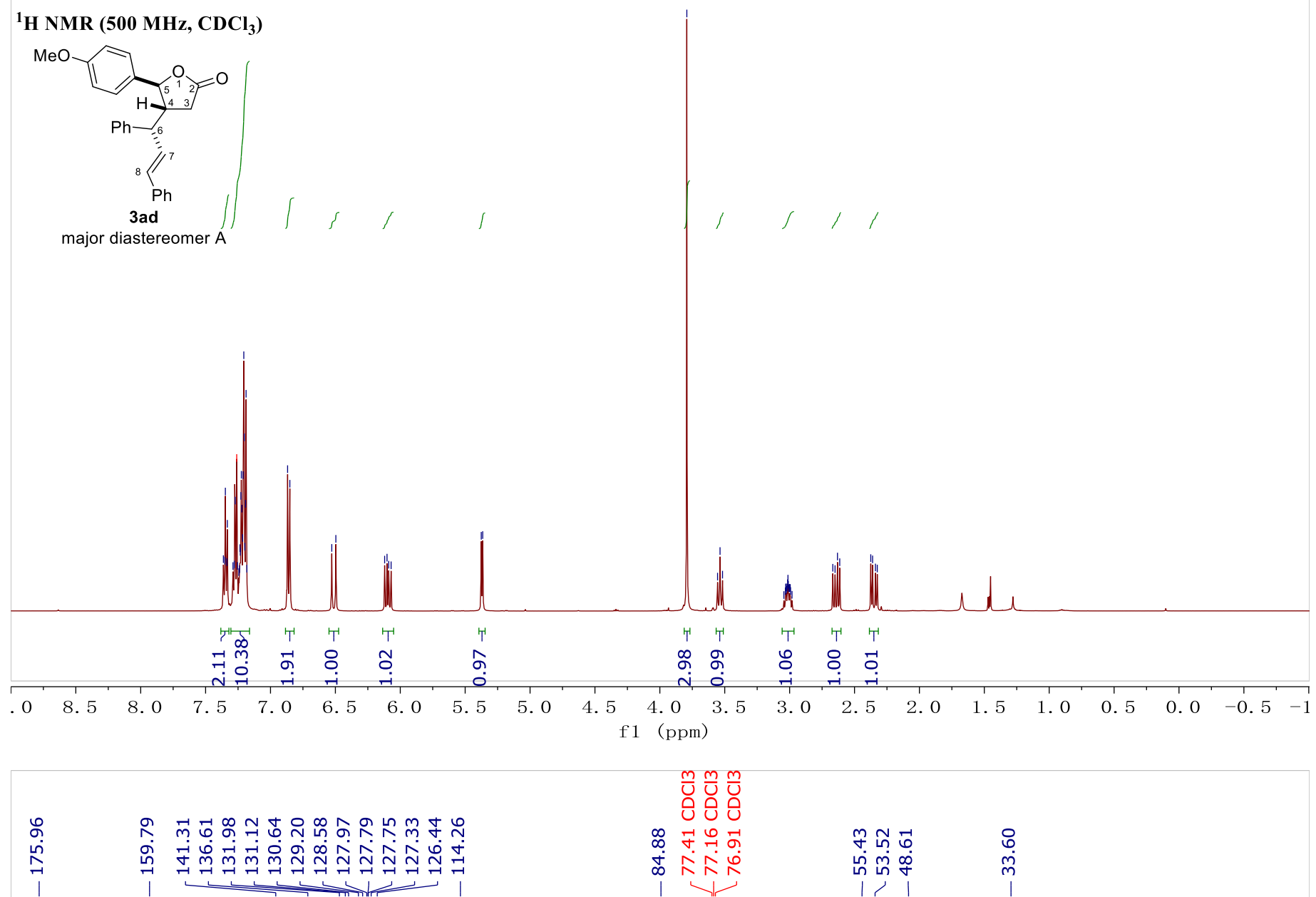

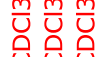

$\infty$ 감바

市ベッ

ซึ กิ

นึ่

1) 1

${ }^{13} \mathrm{C}$ NMR (125 MHz, $\mathrm{CDCl}_{3}$ )
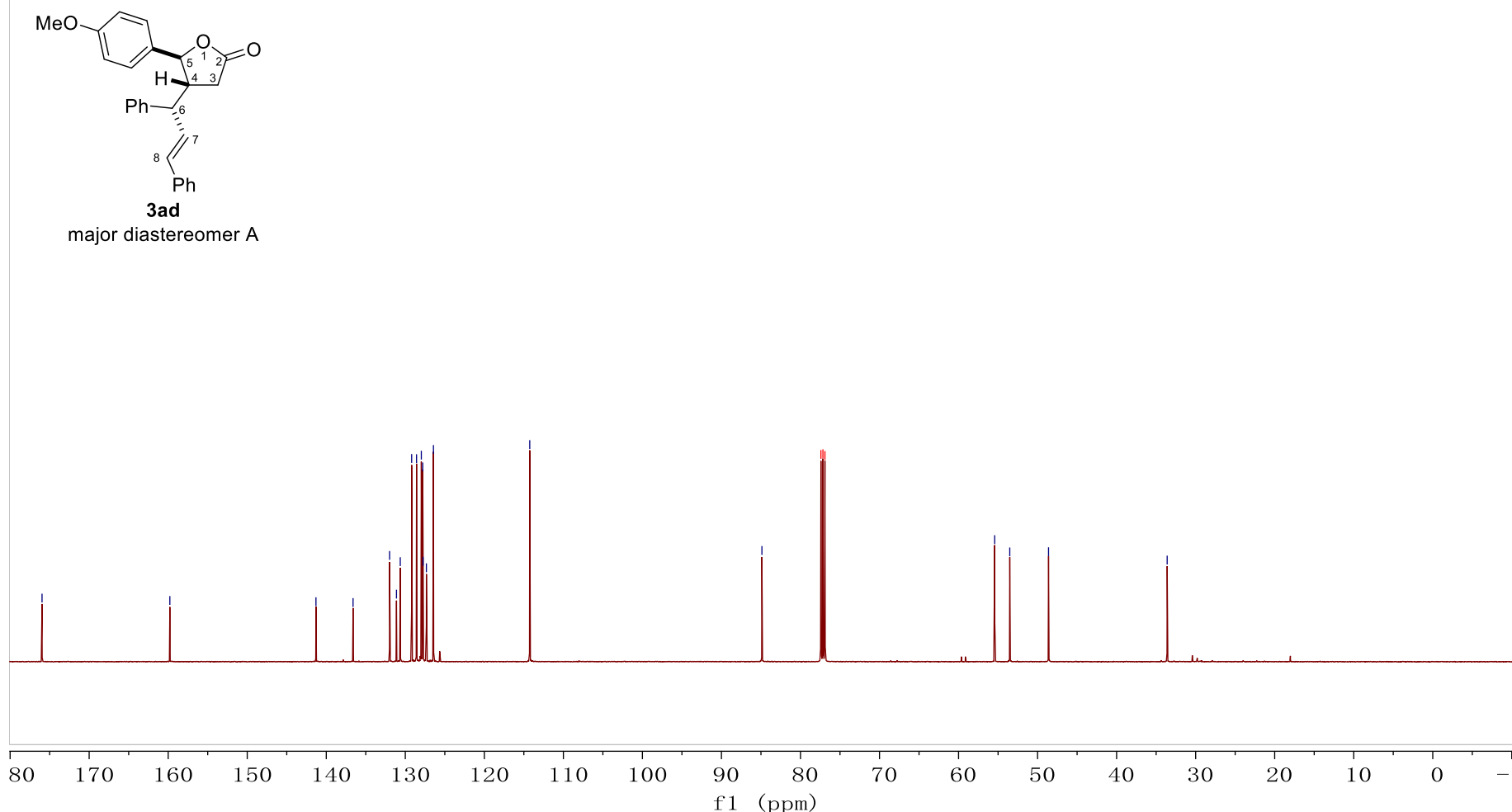


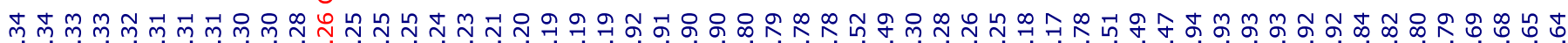

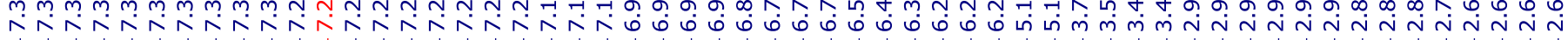

${ }^{1}$ H NMR (500 MHz, $\mathrm{CDCl}_{3}$ )

$\mathrm{MeO}$

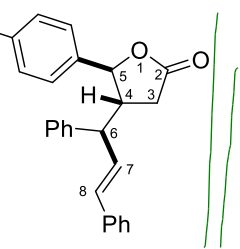

3ad

minor diastereomer $\mathrm{B}$
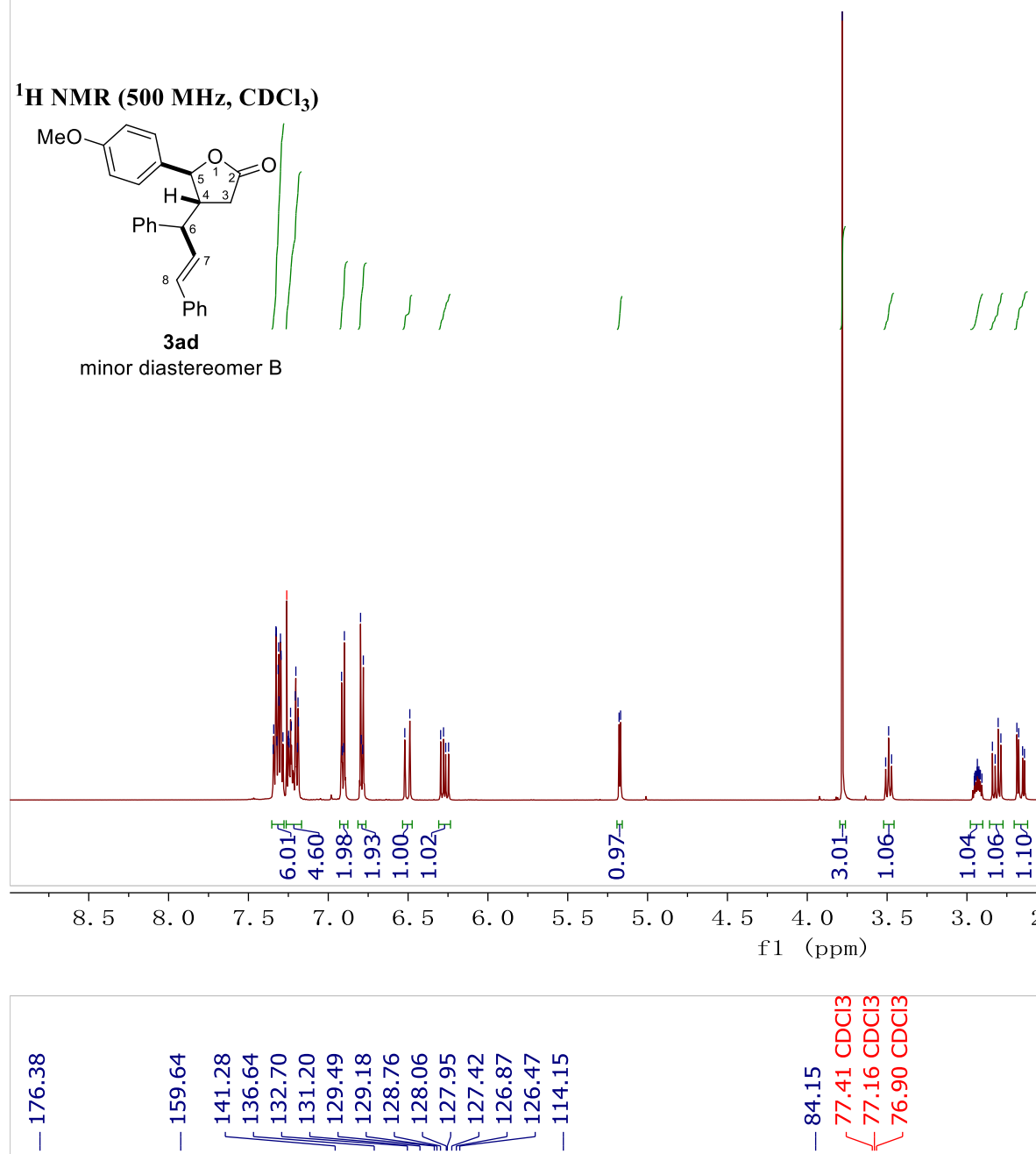

${ }^{13} \mathrm{C}$ NMR (125 MHz, $\mathrm{CDCl}_{3}$ )

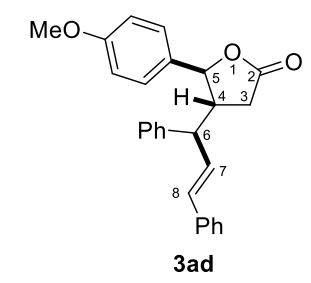

minor diastereomer $\mathrm{B}$

$m \bar{m}$
$\overline{0}$
0
0

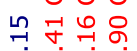

范令官

少我
ᄂ

นึ่

1)
$\stackrel{N}{N}$

( minor diastereomer $B$

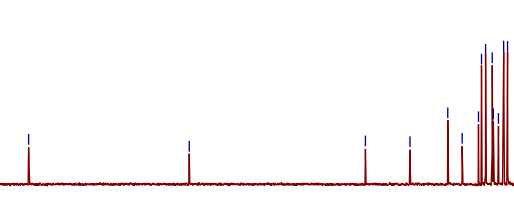


${ }^{1} \mathrm{H}$ NMR (400 MHz, $\mathrm{CDCl}_{3}$ )

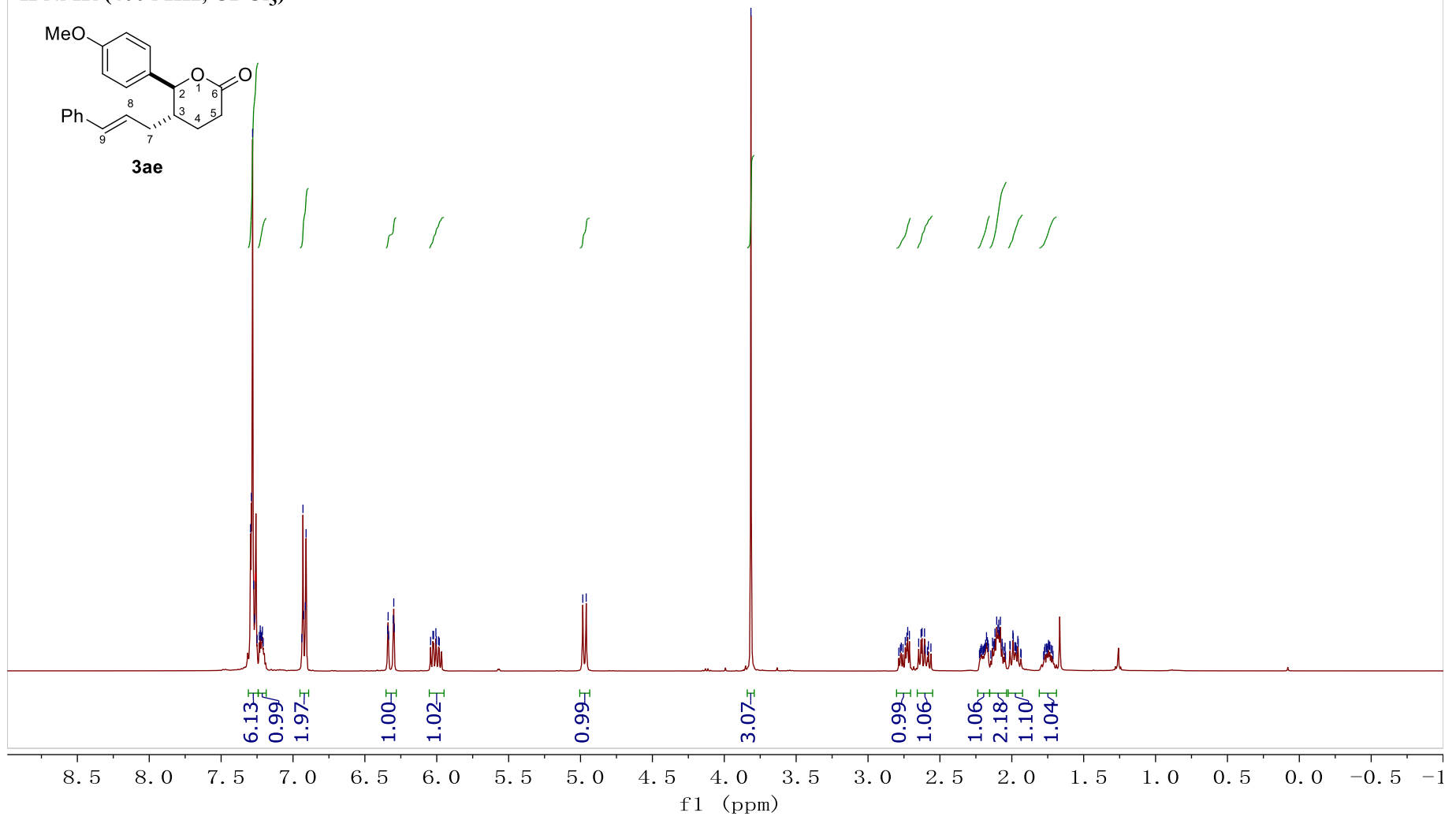

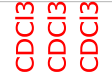

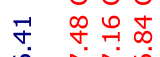

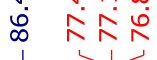

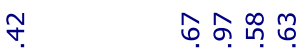

นก่

\section{${ }^{13} \mathrm{C}$ NMR (100 MHz, $\left.\mathrm{CDCl}_{3}\right)$}
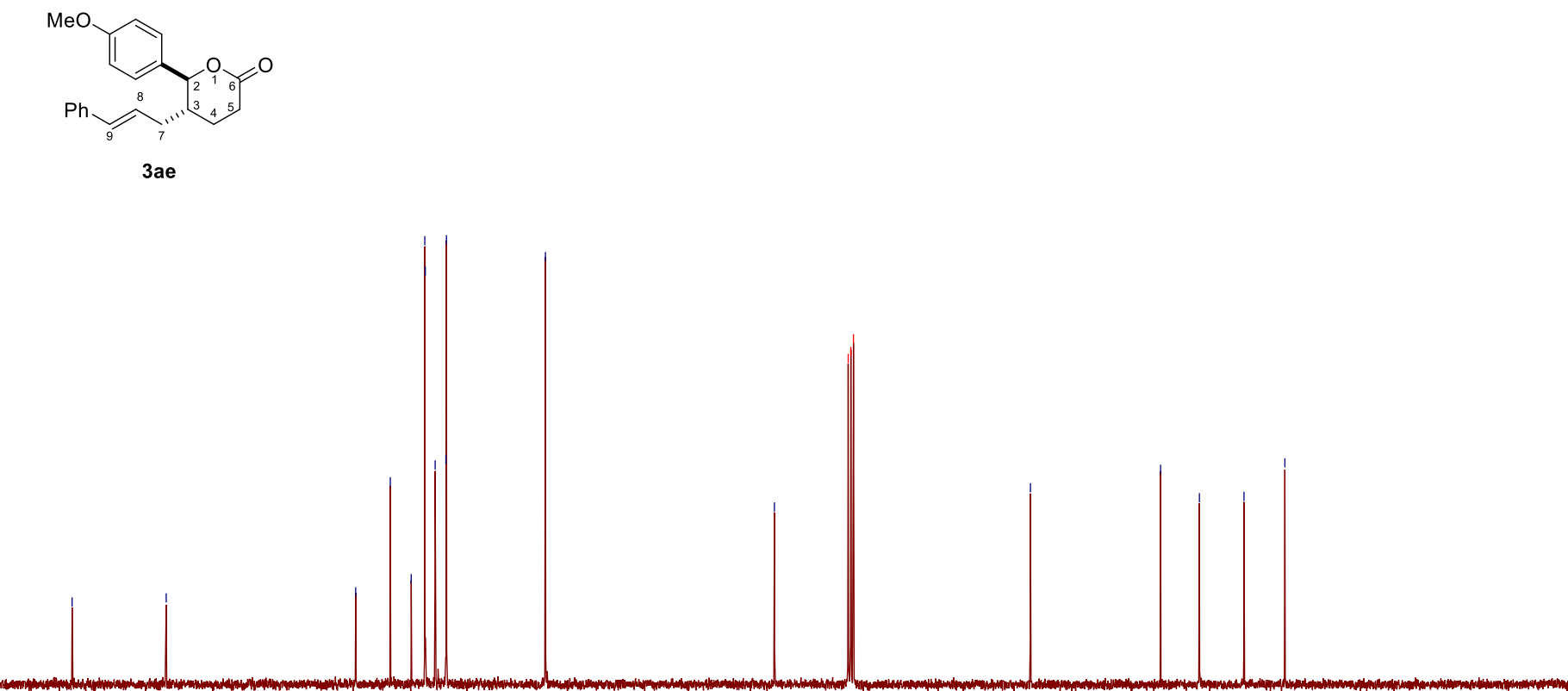


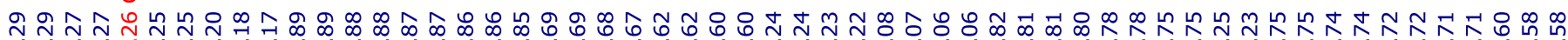

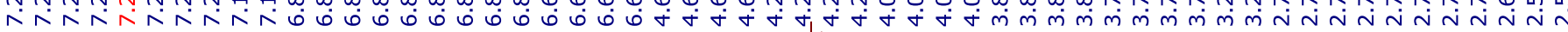

${ }^{1} \mathrm{H}$ NMR (500 MHz, $\left.\mathrm{CDCl}_{3}\right)$
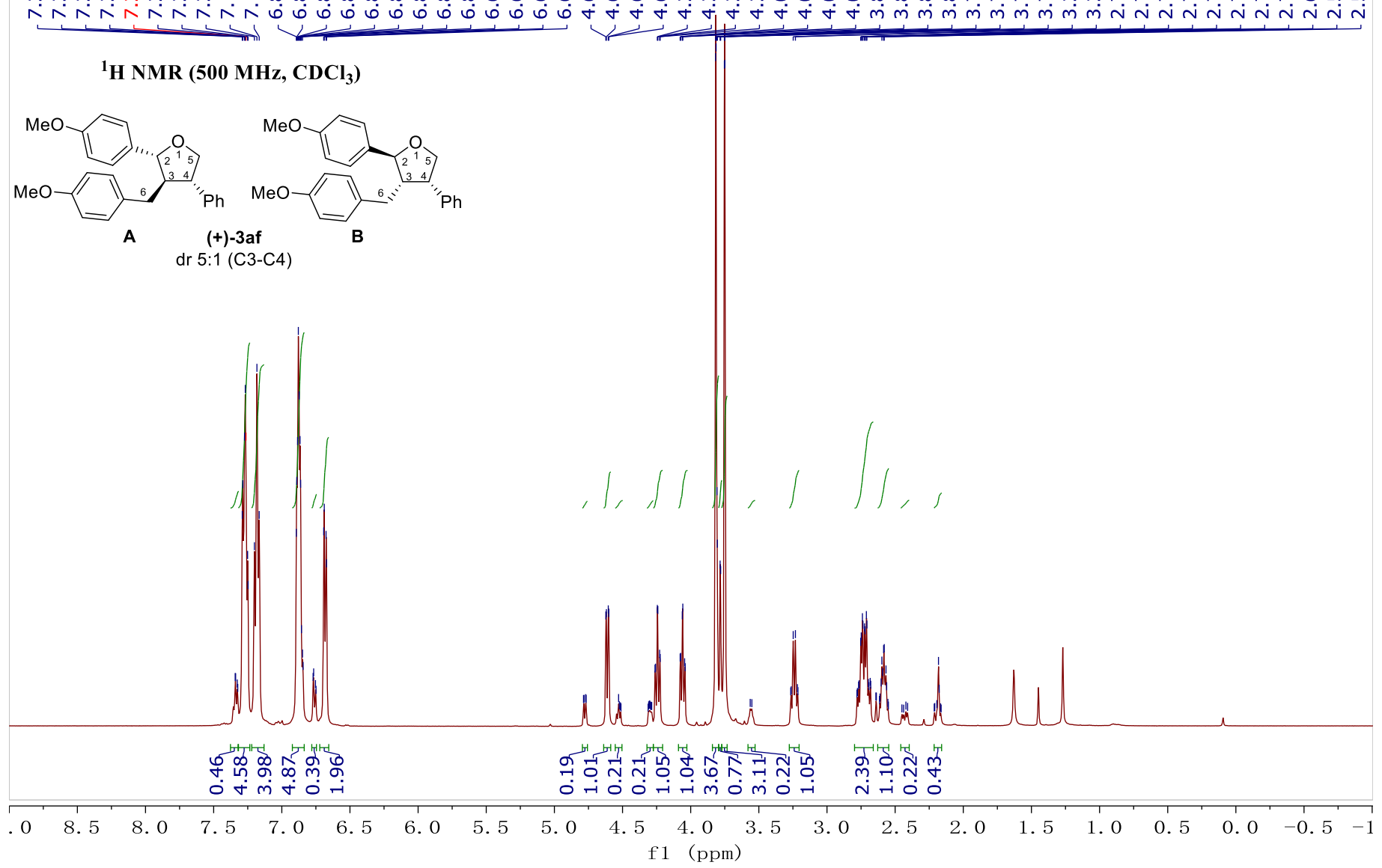

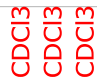

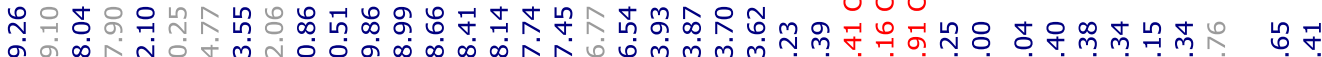

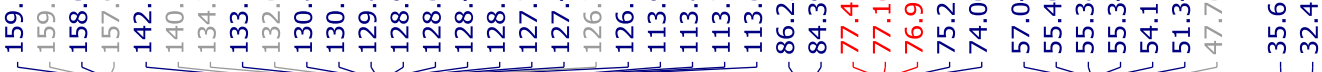

\section{${ }^{13} \mathrm{C}$ NMR (125 MHz, $\left.\mathrm{CDCl}_{3}\right)$}
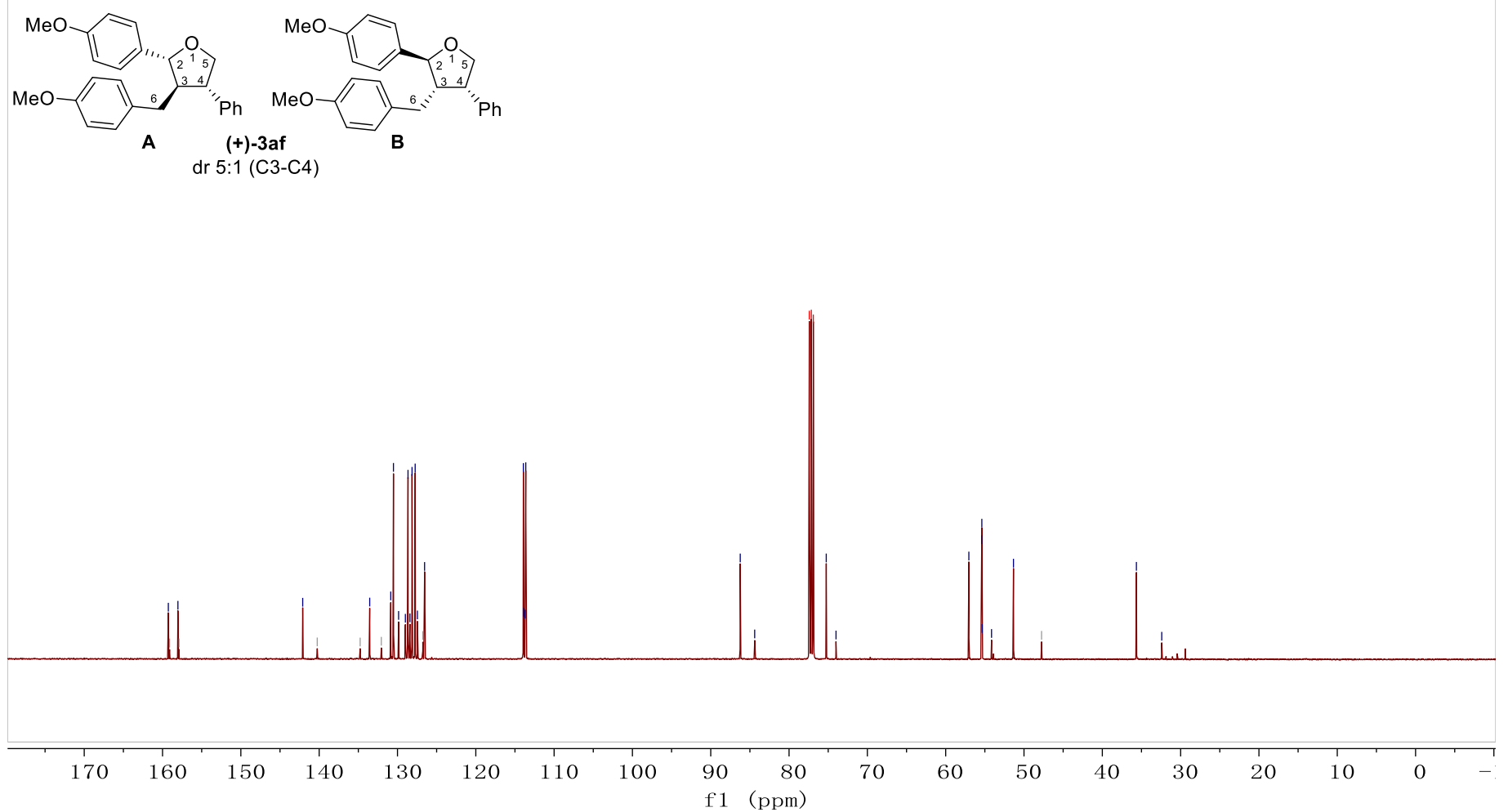
岂

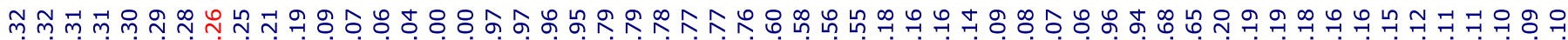

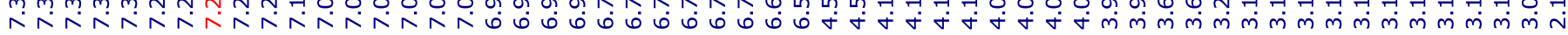

${ }^{1} \mathrm{H}$ NMR (500 MHz, $\mathrm{CDCl}_{3}$ )

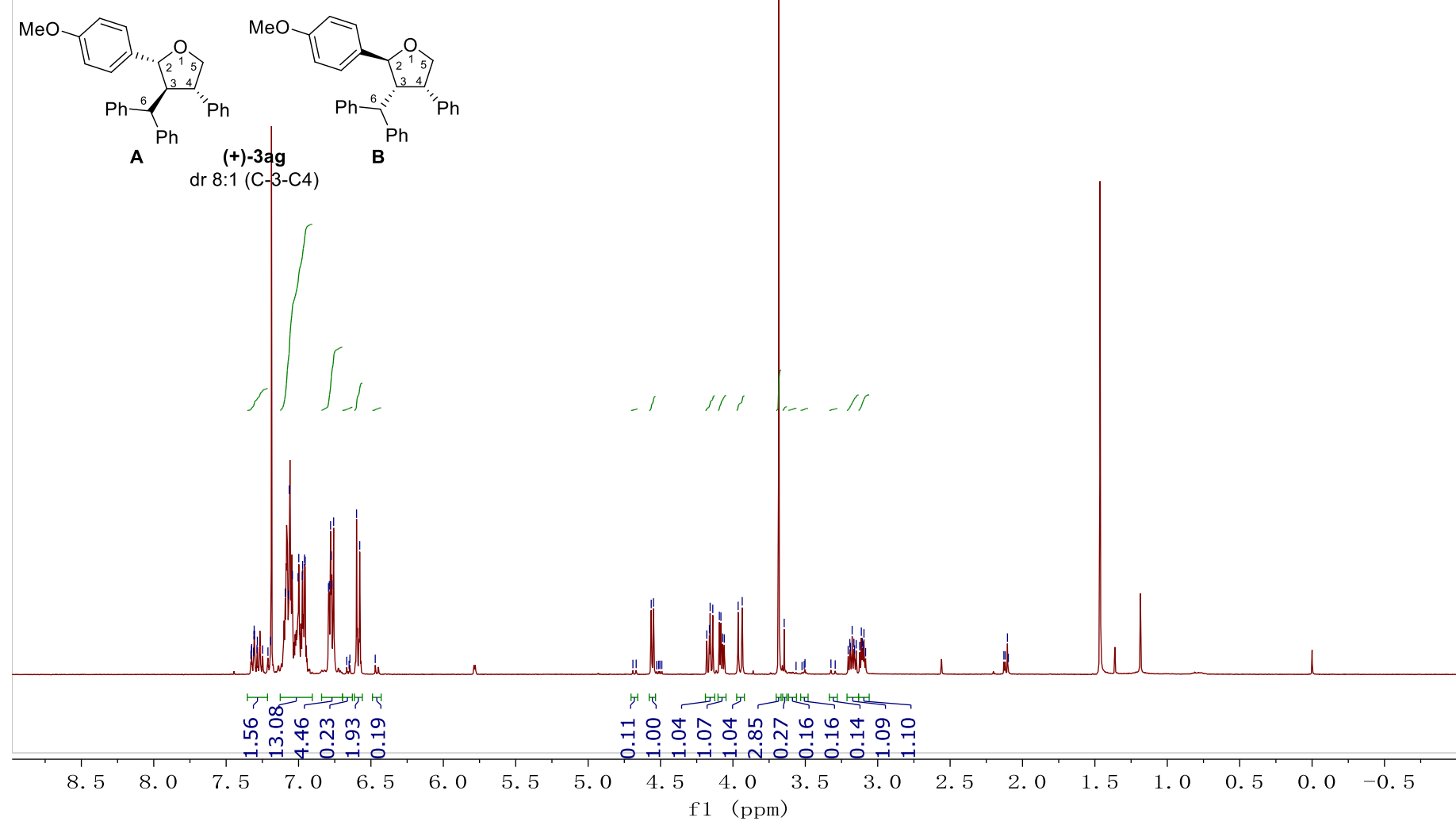

\section{$\overline{\mathrm{U}} \frac{m}{\mathrm{U}} \frac{\mathrm{m}}{\mathrm{U}}$}

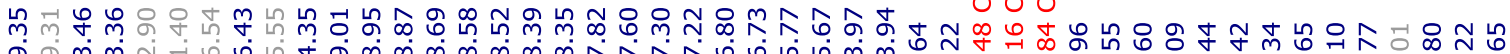

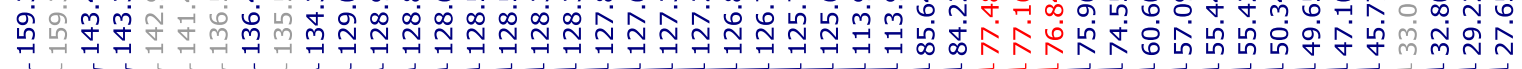

\section{${ }^{13} \mathrm{C}$ NMR (125 MHz, $\left.\mathrm{CDCl}_{3}\right)$}
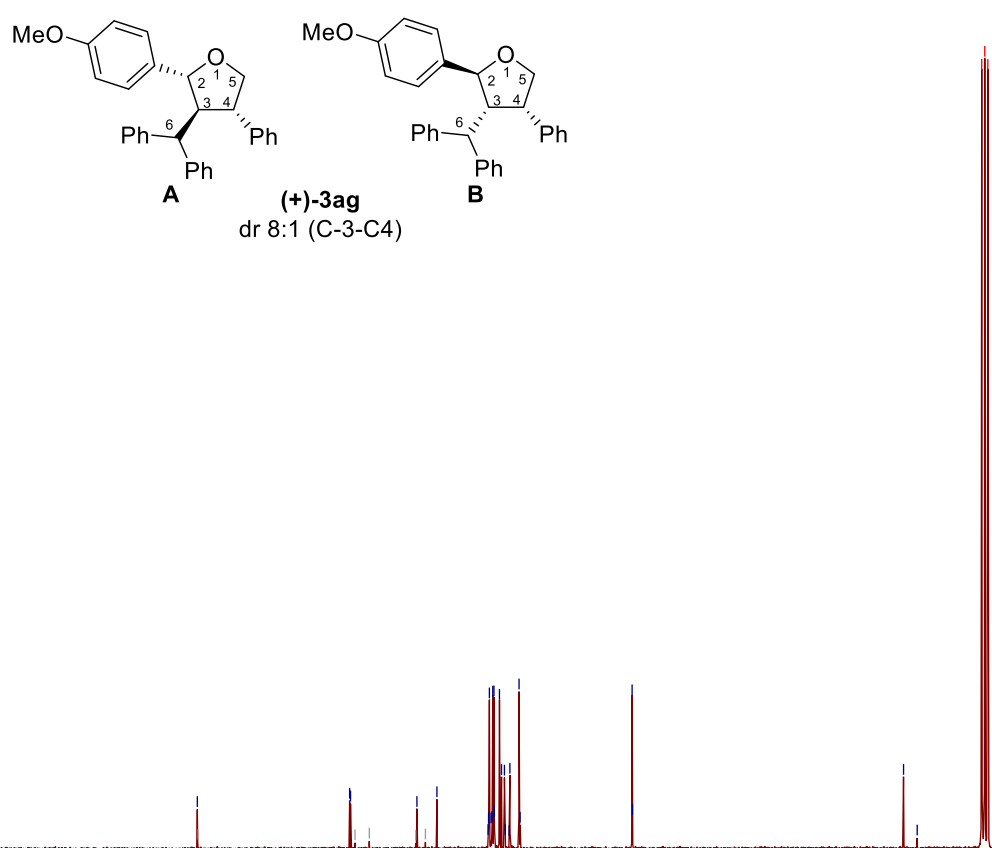
${ }^{1} \mathrm{H}$ - ${ }^{1} \mathrm{H}$ COSY - $90\left(500 \mathrm{MHz}, \mathrm{CDCl}_{3}\right)$

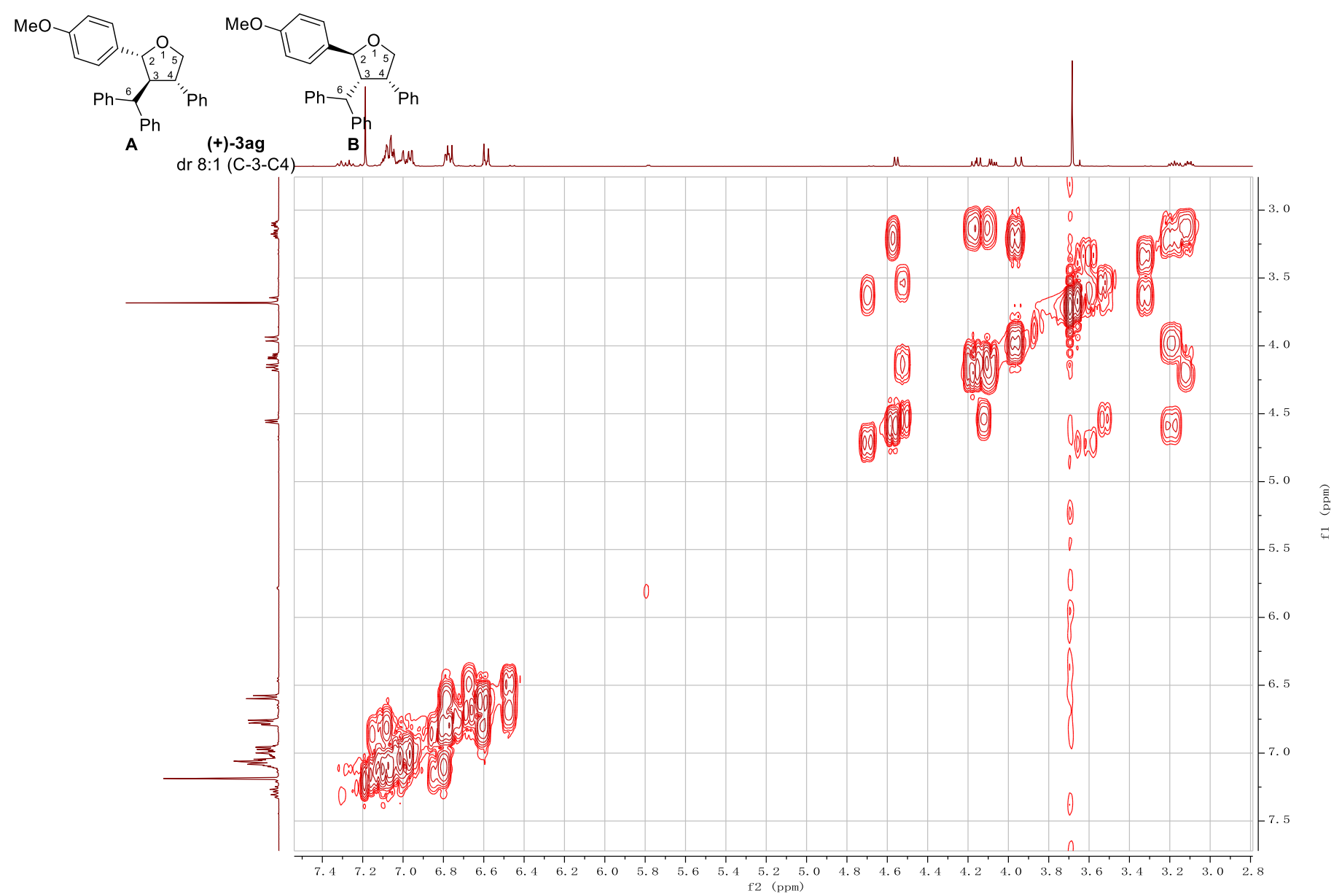

${ }^{1} \mathrm{H}(500 \mathrm{MHz})-{ }^{13} \mathrm{C}(125 \mathrm{MHz}) \mathrm{HSQC}\left(\mathrm{CDCl}_{3}\right)$

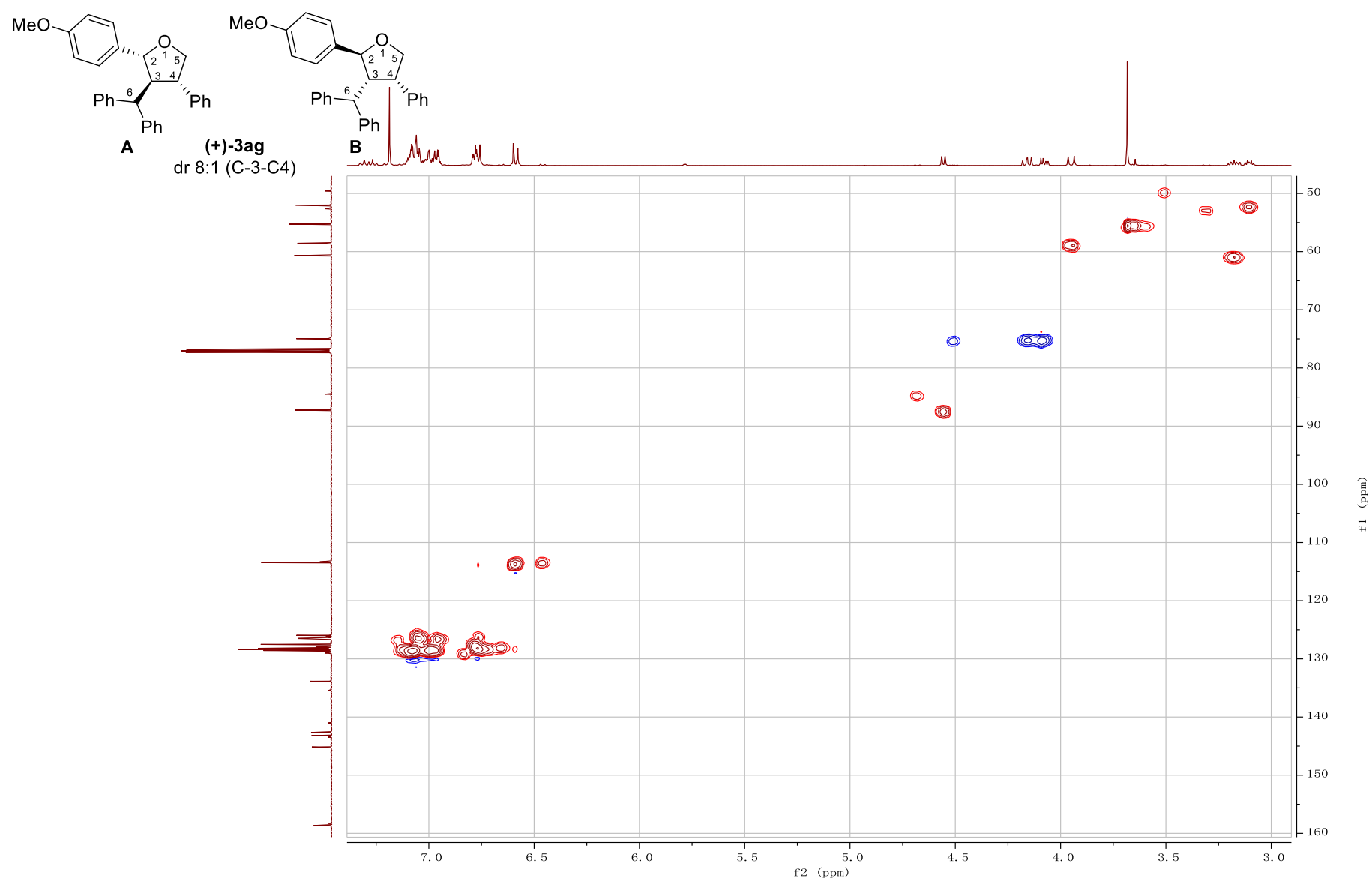

S64 
NOESY-2D (500 MHz, $\left.\mathrm{CDCl}_{3}\right)$

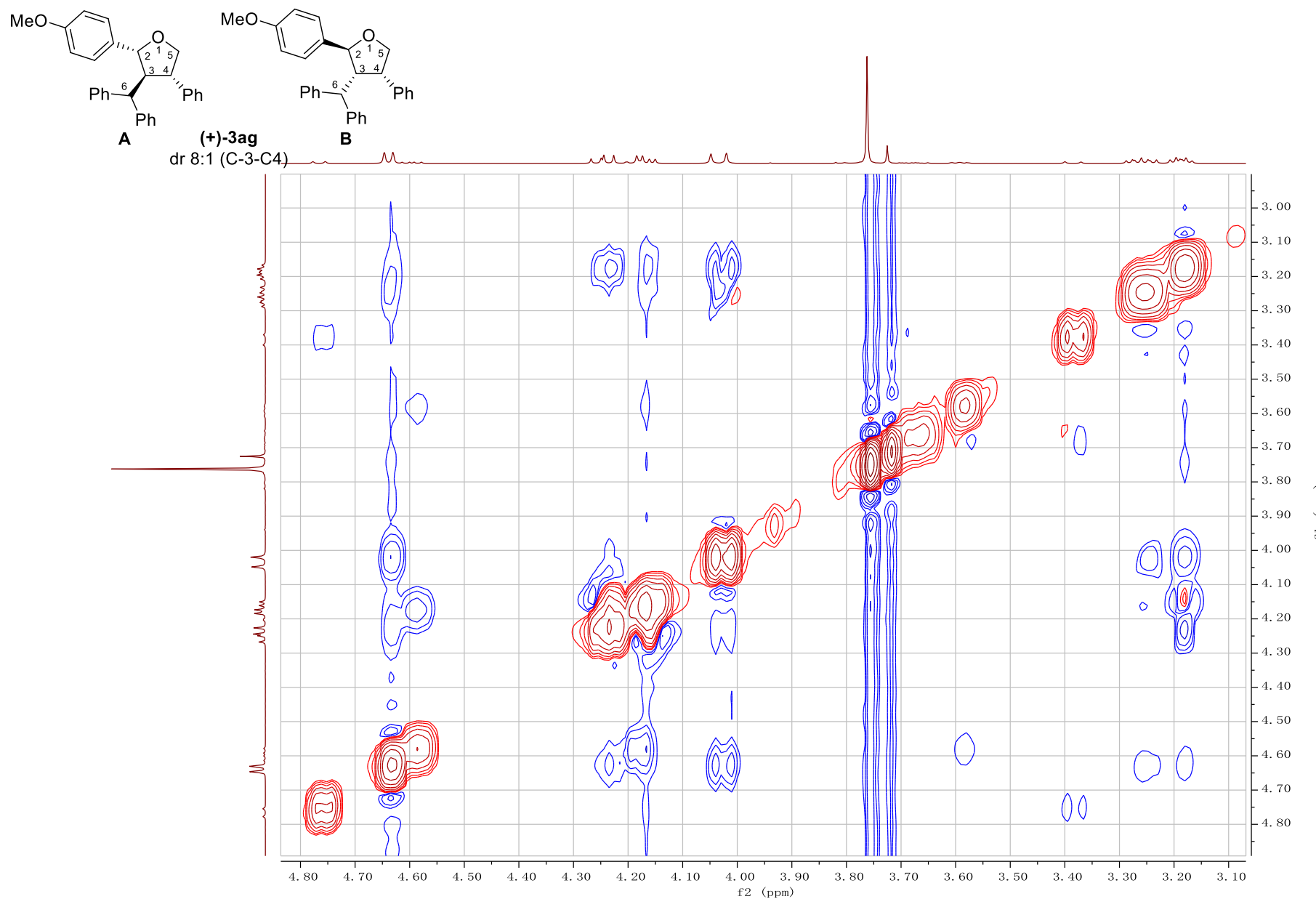


岗

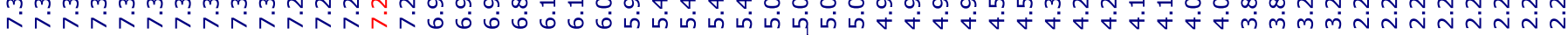

${ }^{1} \mathrm{H}$ NMR (500 MHz, $\mathrm{CDCl}_{3}$ )
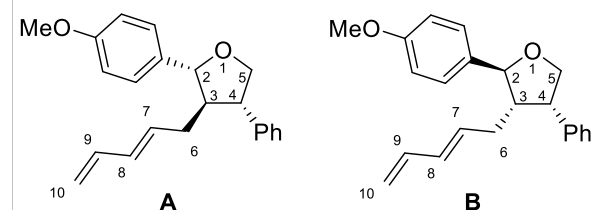

(+)-3ah dr 5:1 (C3-C4)

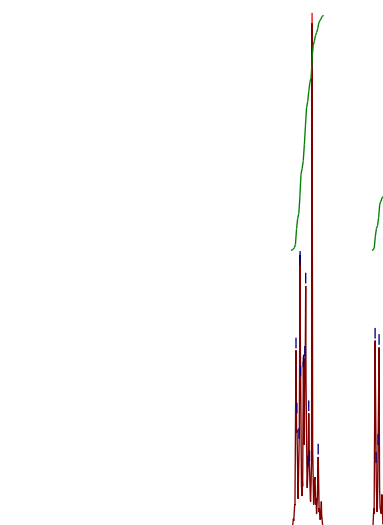

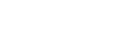

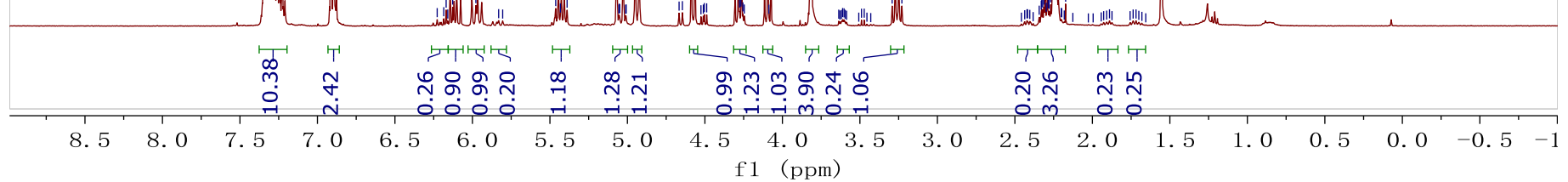

\section{产商商}

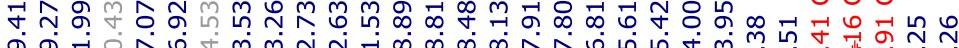

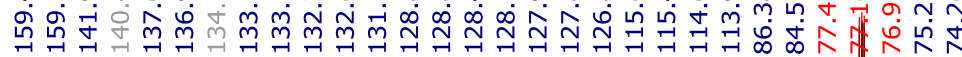

${ }^{13} \mathrm{C}$ NMR (125 MHz, $\left.\mathrm{CDCl}_{3}\right)$
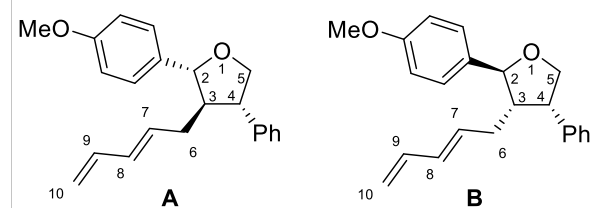

(+)-3ah dr 5:1 (C3-C4)

눈 눙ำ

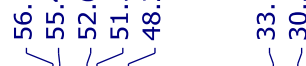

뭉
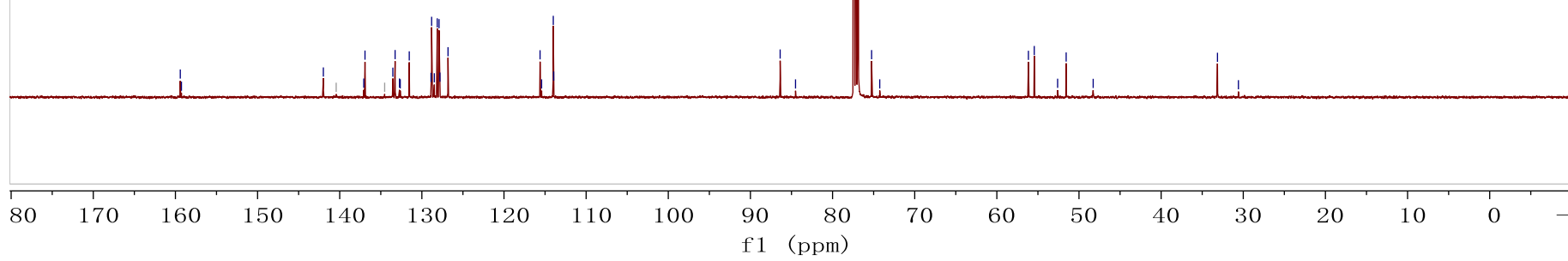
m

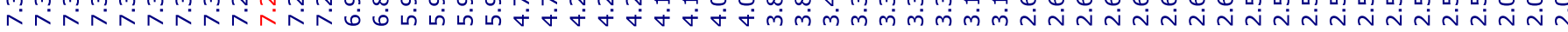

${ }^{1} \mathrm{H}$ NMR $\left(400 \mathrm{MHz}, \mathrm{CDCl}_{3}\right)$

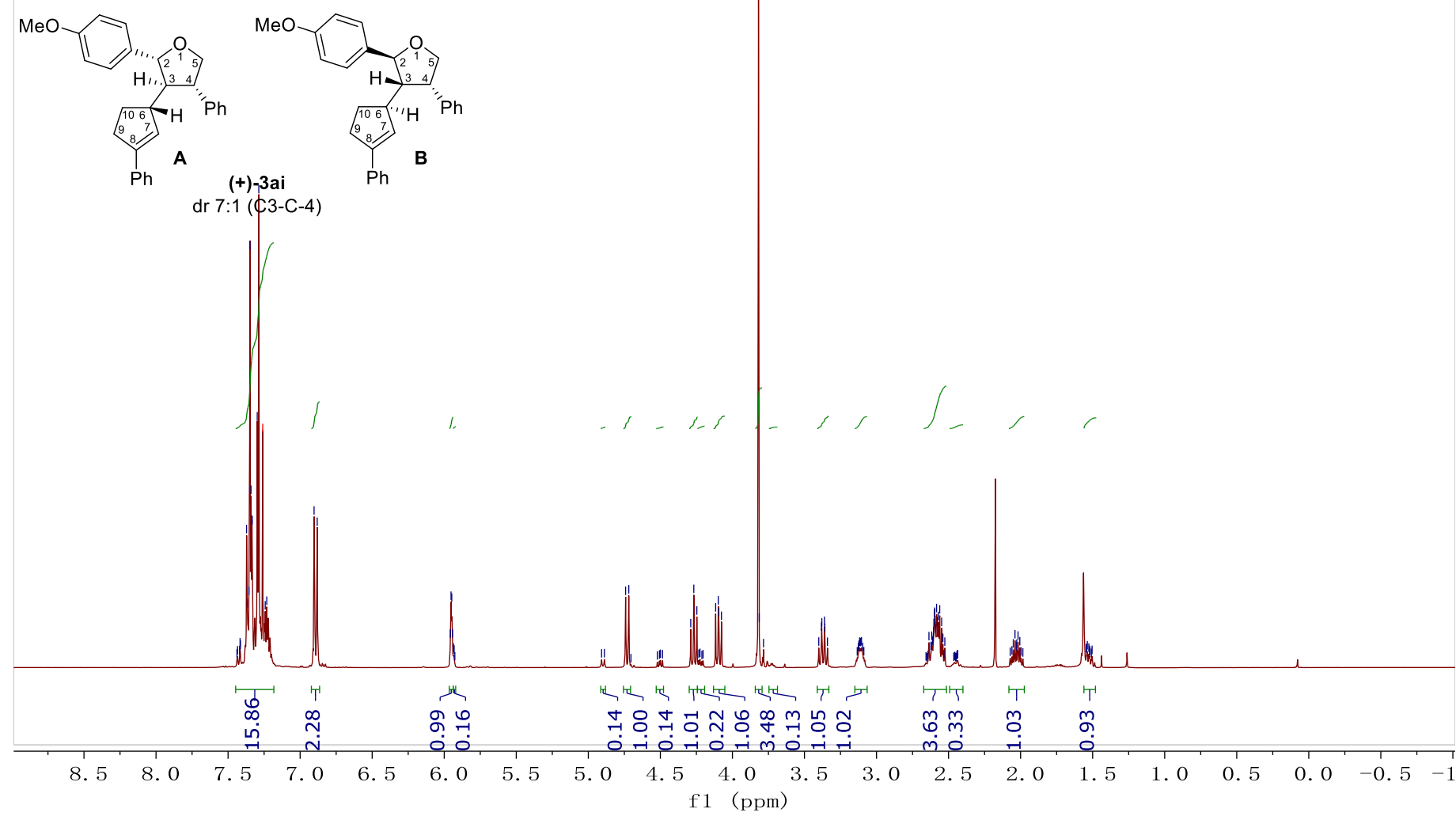

${ }^{13} \mathrm{C}$ NMR (125 MHz, $\left.\mathrm{CDCl}_{3}\right)$
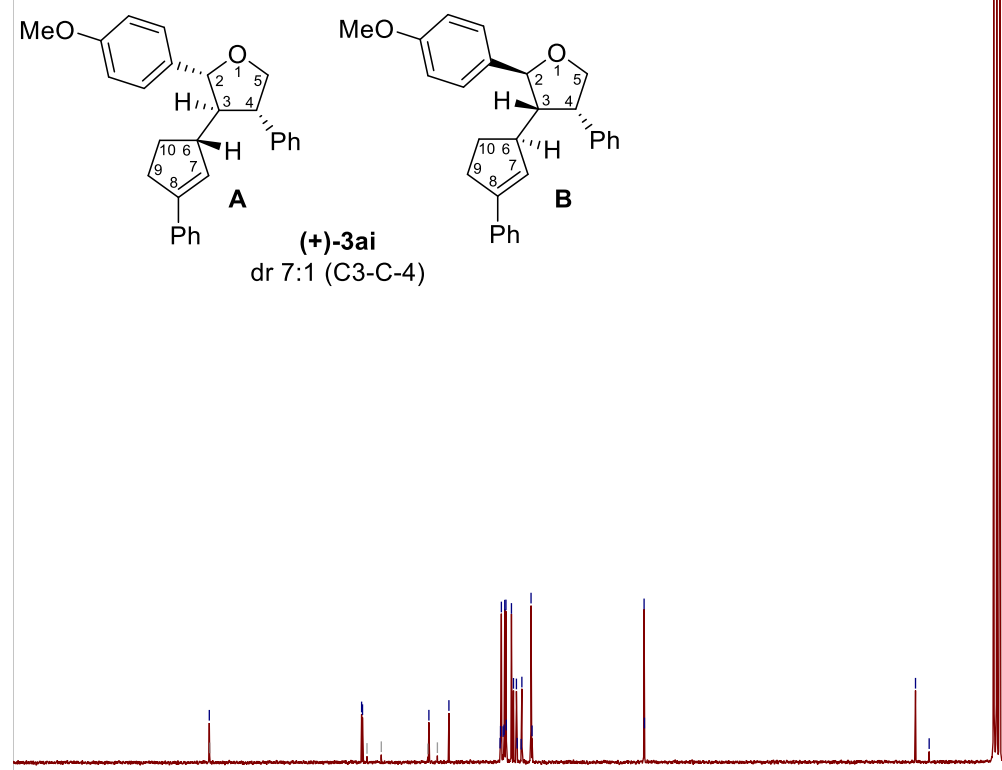


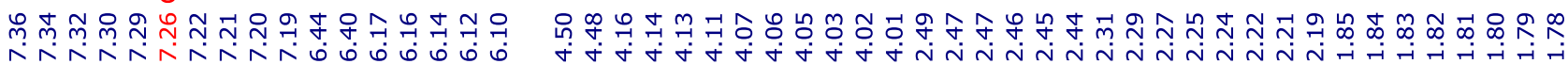

${ }^{1} \mathrm{H}$ NMR (400 MHz, $\mathrm{CDCl}_{3}$ )

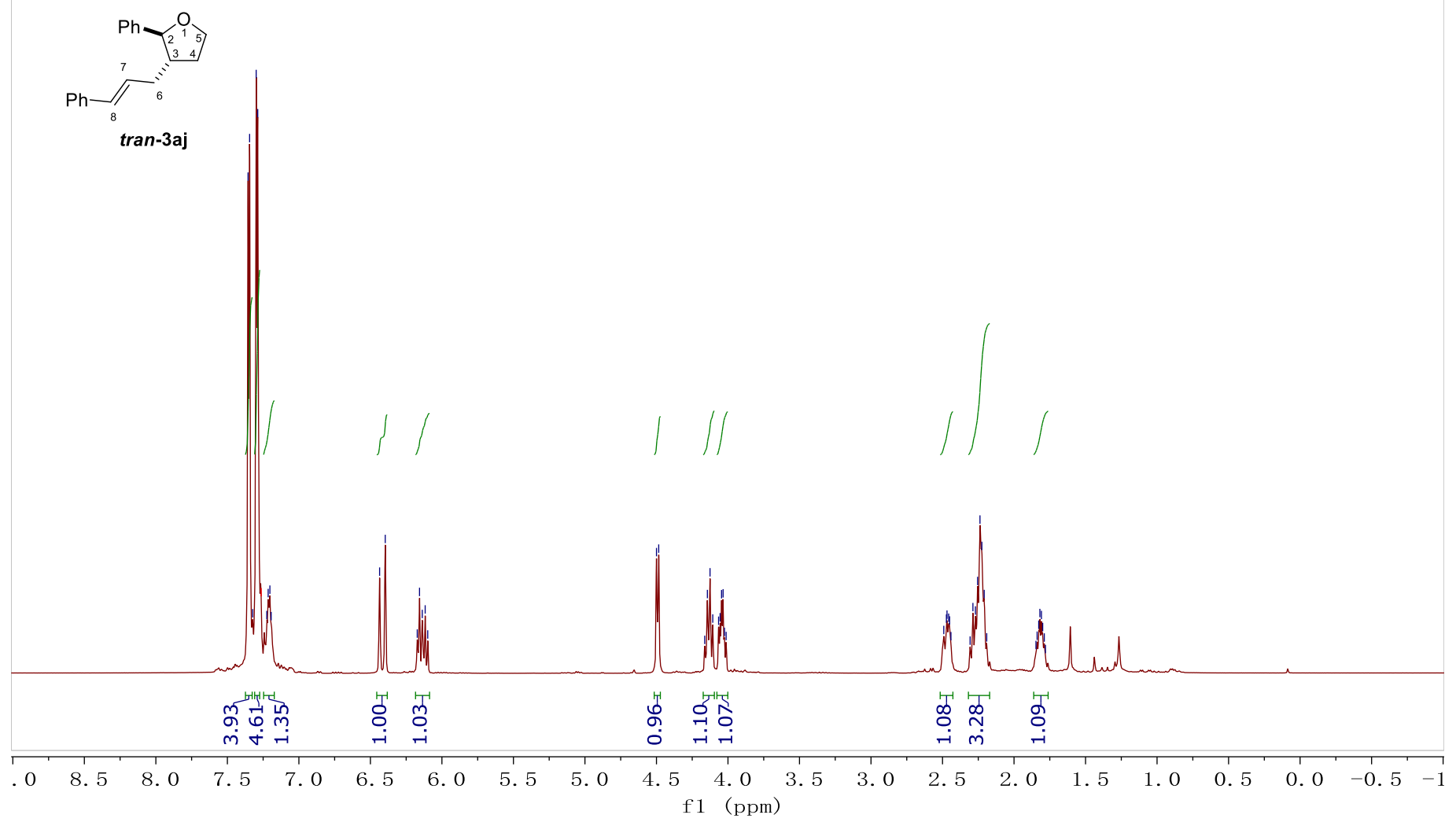

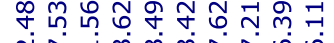

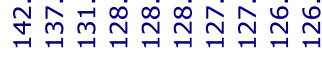

${ }^{13} \mathrm{C}$ NMR (100 MHz, $\left.\mathrm{CDCl}_{3}\right)$

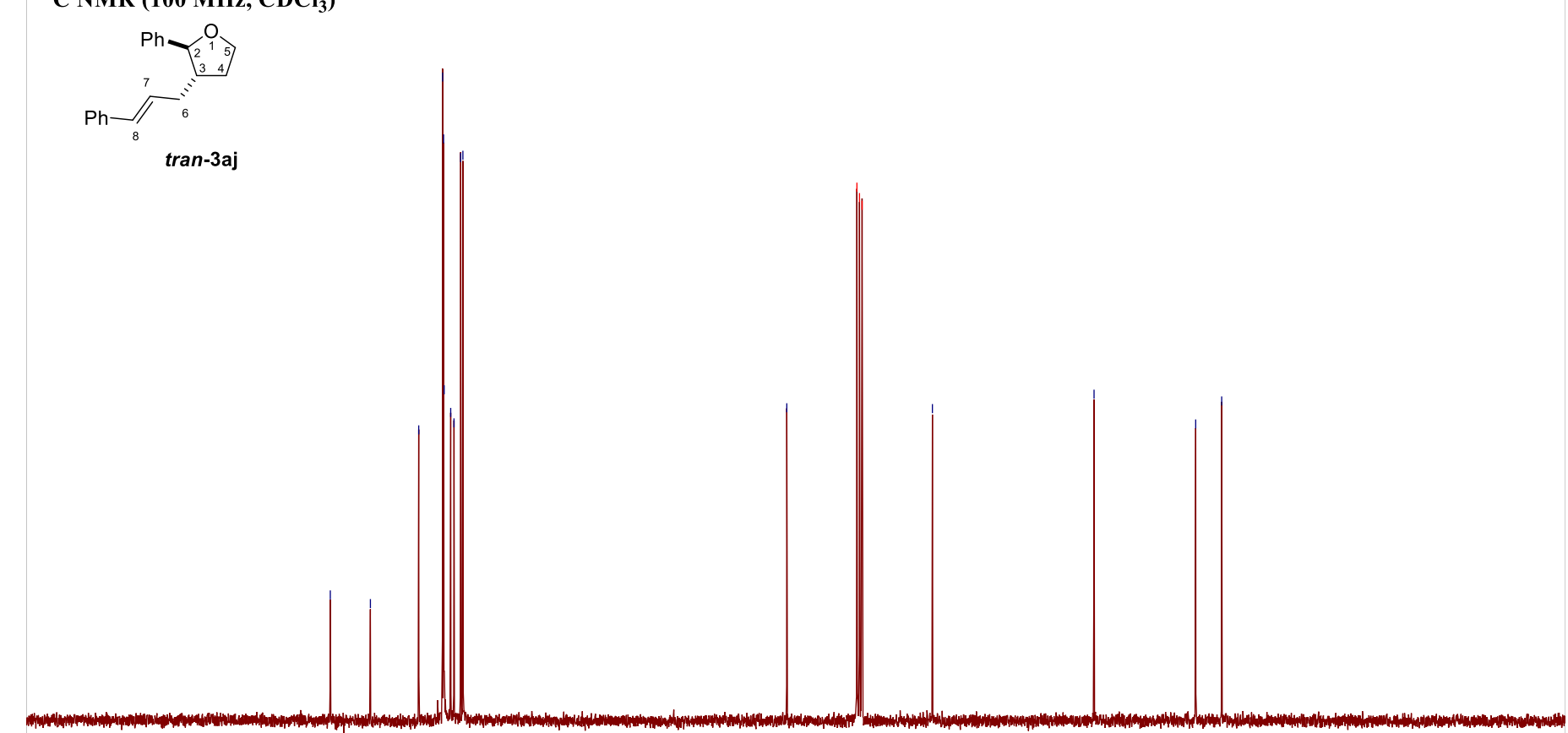

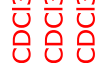

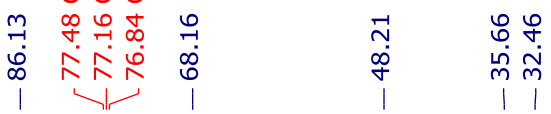

$\begin{array}{llllllllll}170 & 160 & 150 & 140 & 130 & 120 & 110 & 100 & 90 & 80\end{array}$ 


\section{${ }^{1} \mathrm{H}$ NMR (400 MHz, $\left.\mathrm{CDCl}_{3}\right)$}

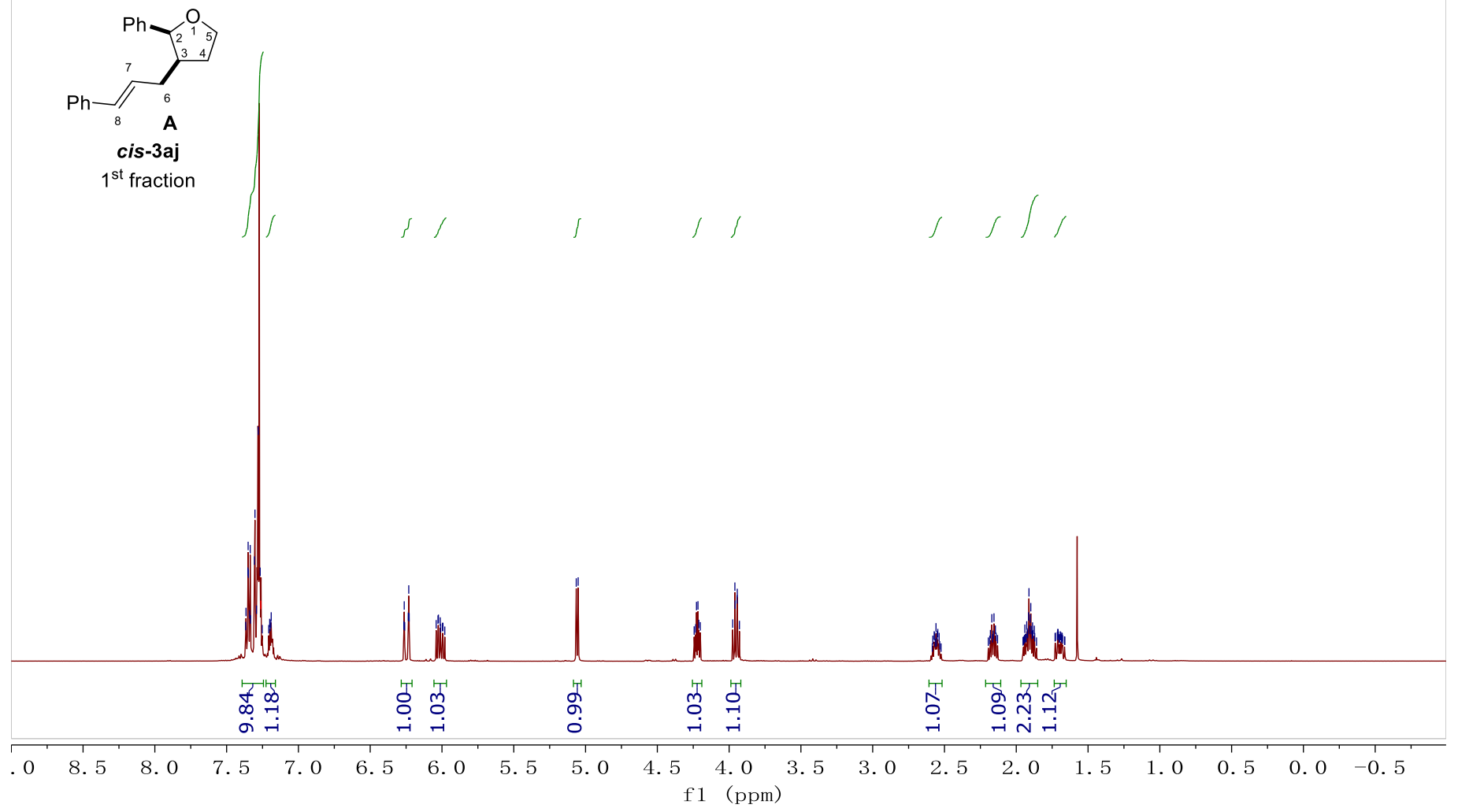

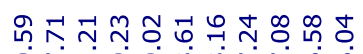

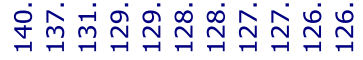

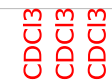

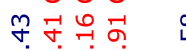

miñ

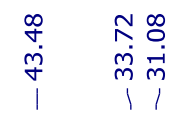

\section{${ }^{13} \mathrm{C}$ NMR (100 MHz, $\left.\mathrm{CDCl}_{3}\right)$}
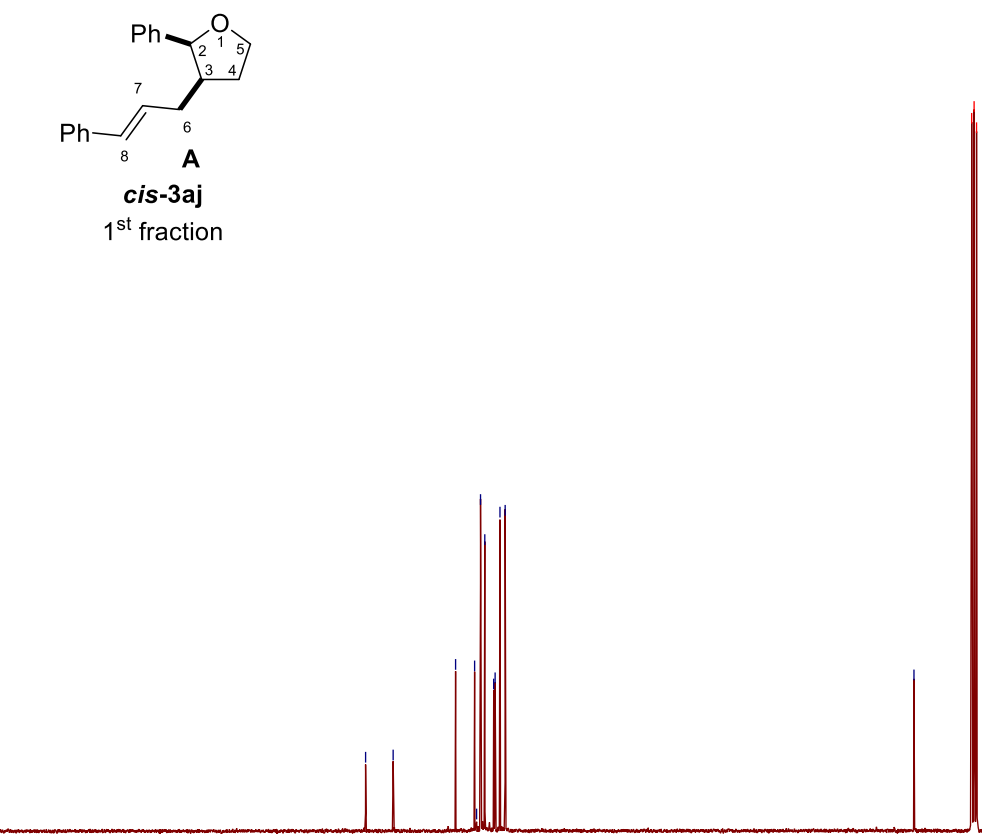


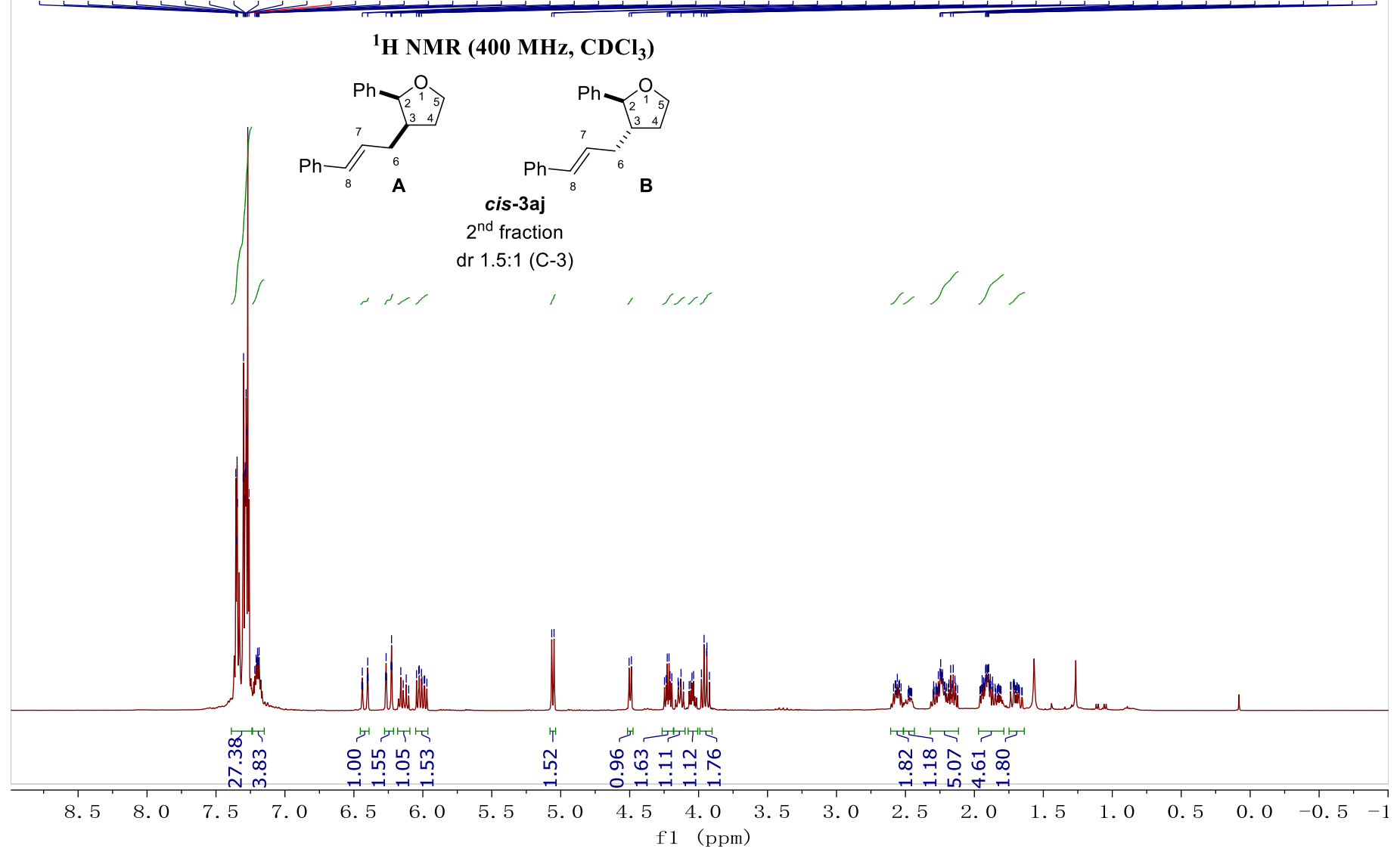

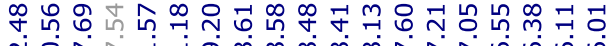

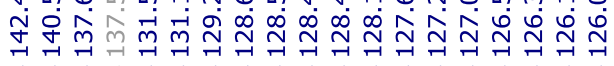

\section{${ }^{13} \mathrm{C}$ NMR (100 MHz, $\mathrm{CDCl}_{3}$ )}

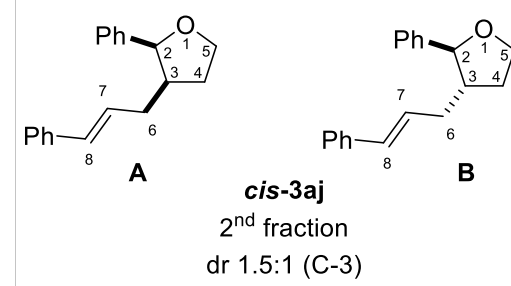

\section{는}

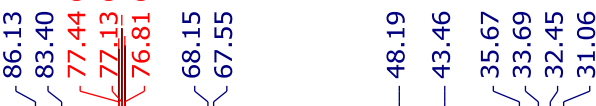
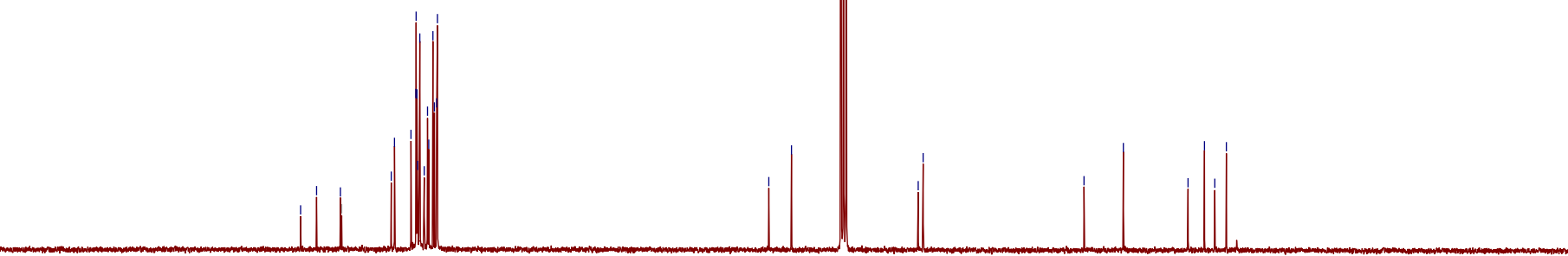
${ }^{1} \mathrm{H}$ - ${ }^{1} \mathrm{H}$ COSY - $90\left(400 \mathrm{MHz}, \mathrm{CDCl}_{3}\right)$
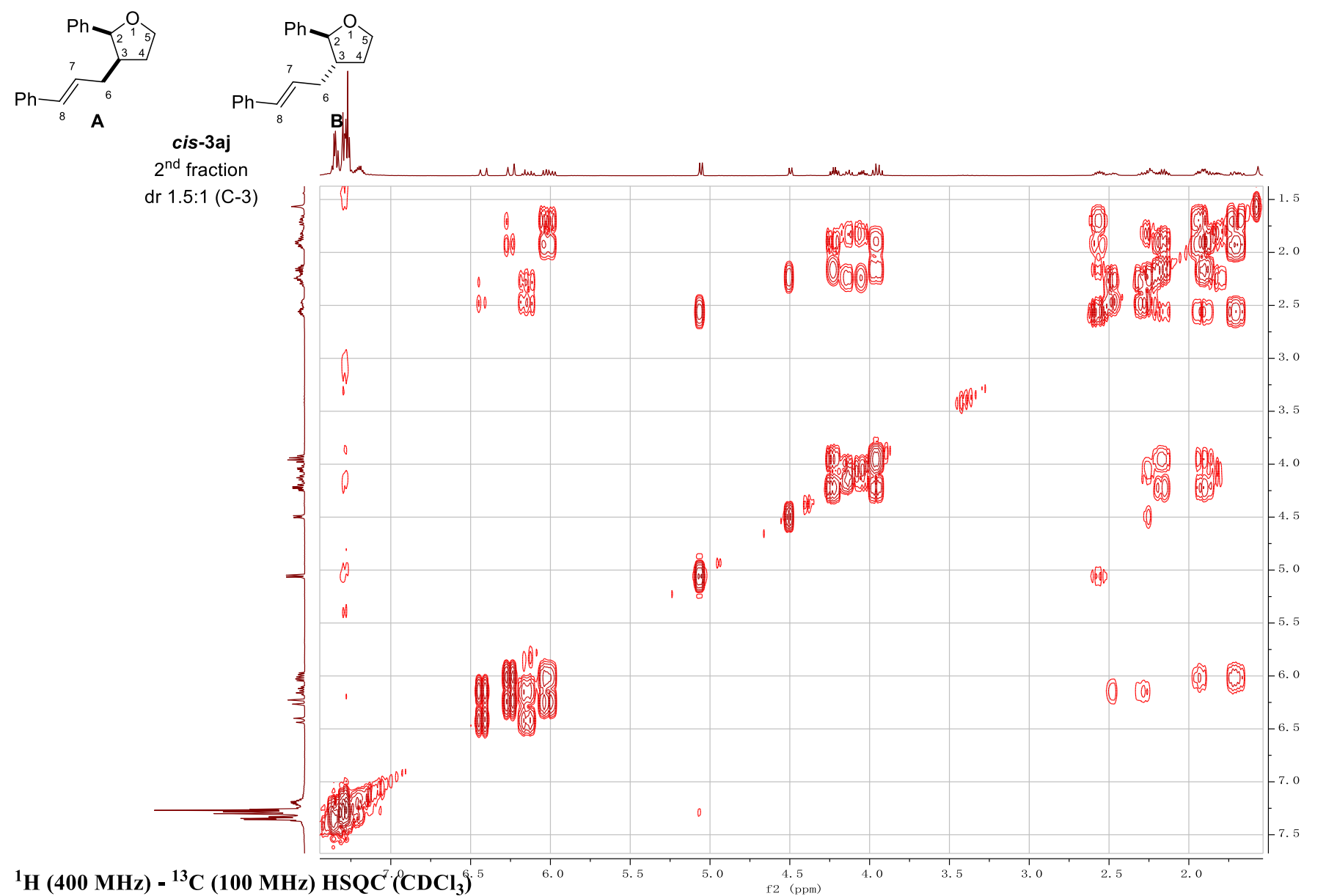

${ }^{1} \mathrm{H}(400 \mathrm{MHz})-{ }^{13} \mathrm{C}(100 \mathrm{MHz}) \mathrm{HSQC}^{1}\left(\mathrm{CDCl}_{3}\right)^{5}$
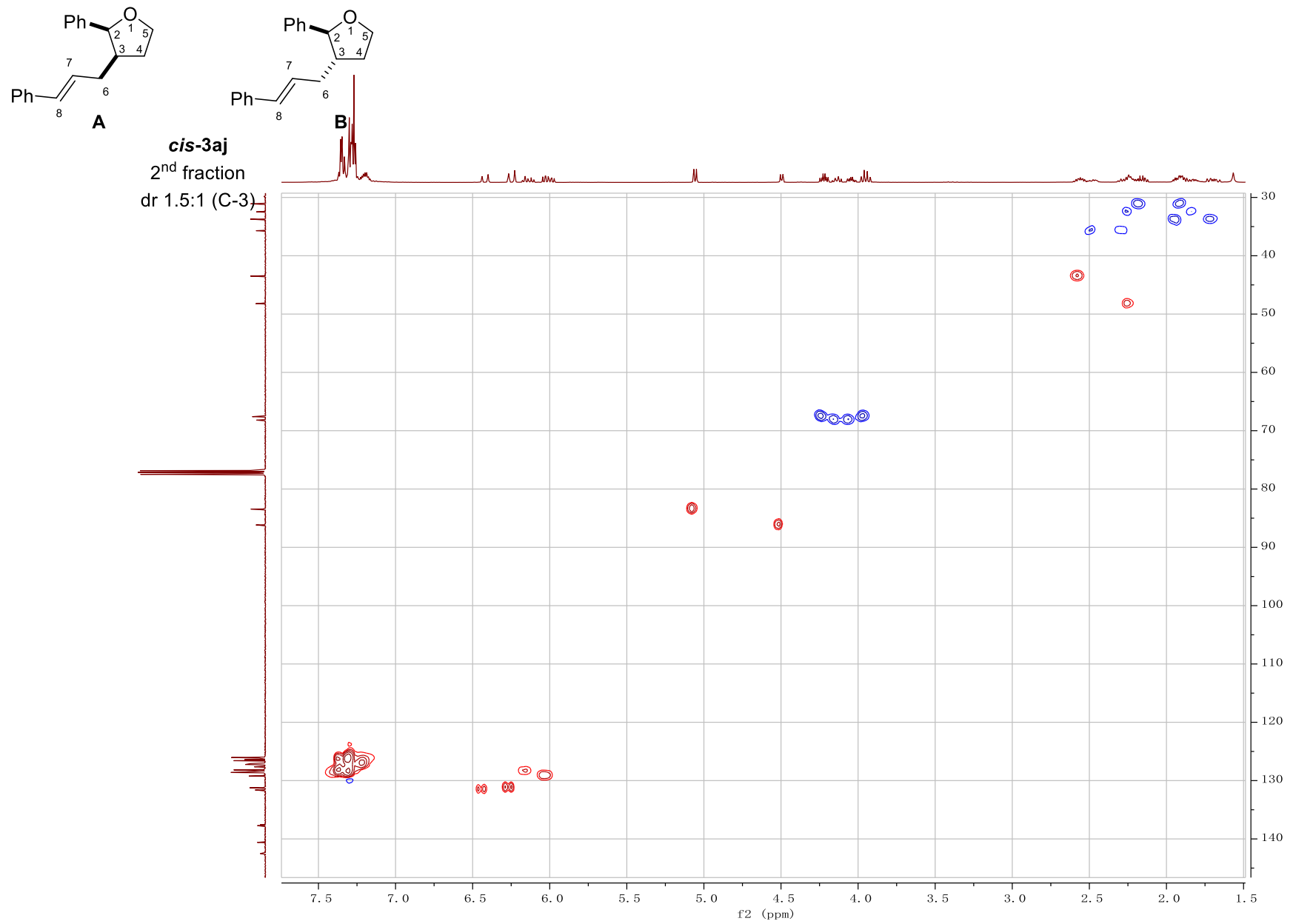


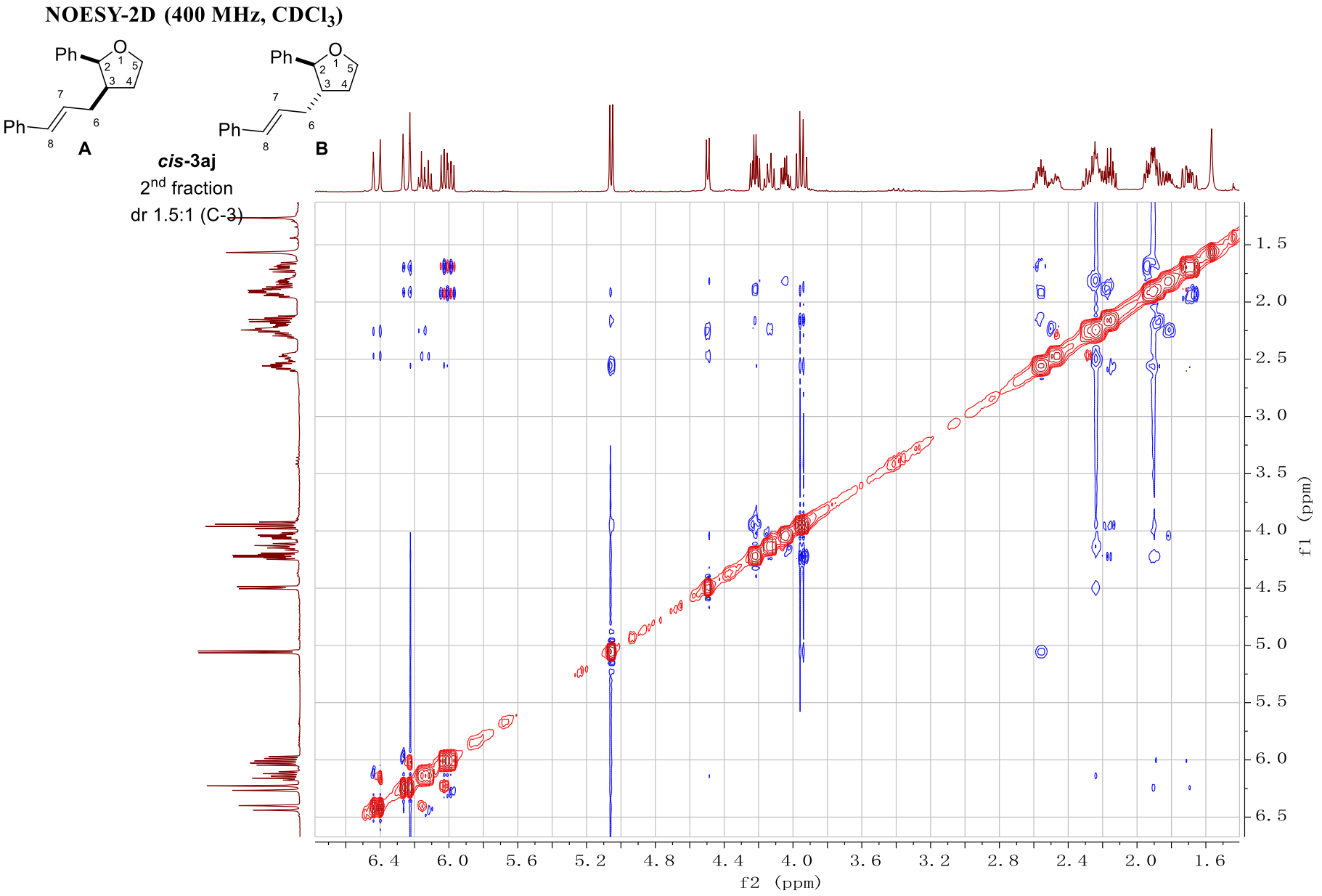


鱼

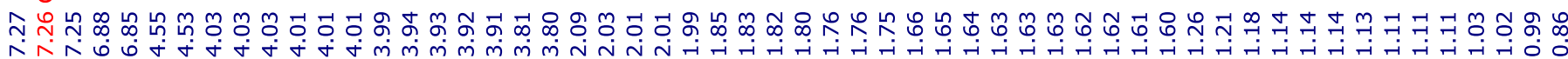

${ }^{1} \mathrm{H}$ NMR (400 MHz, $\left.\mathrm{CDCl}_{3}\right)$

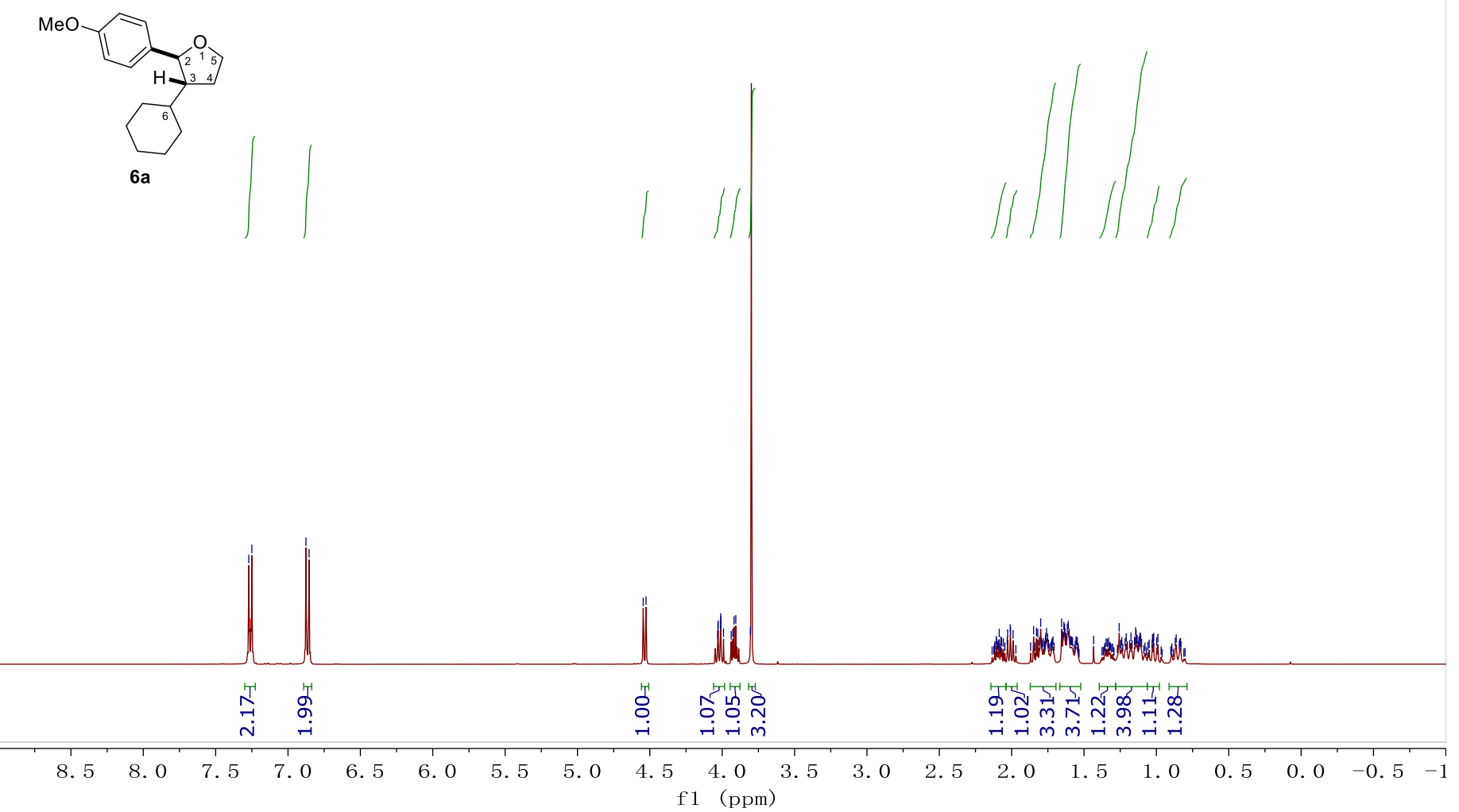

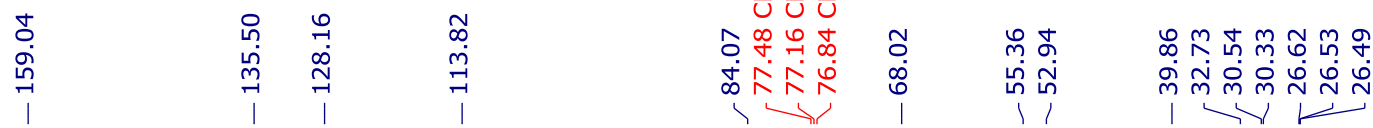

\section{${ }^{13} \mathrm{C}$ NMR (100 MHz, $\left.\mathrm{CDCl}_{3}\right)$}

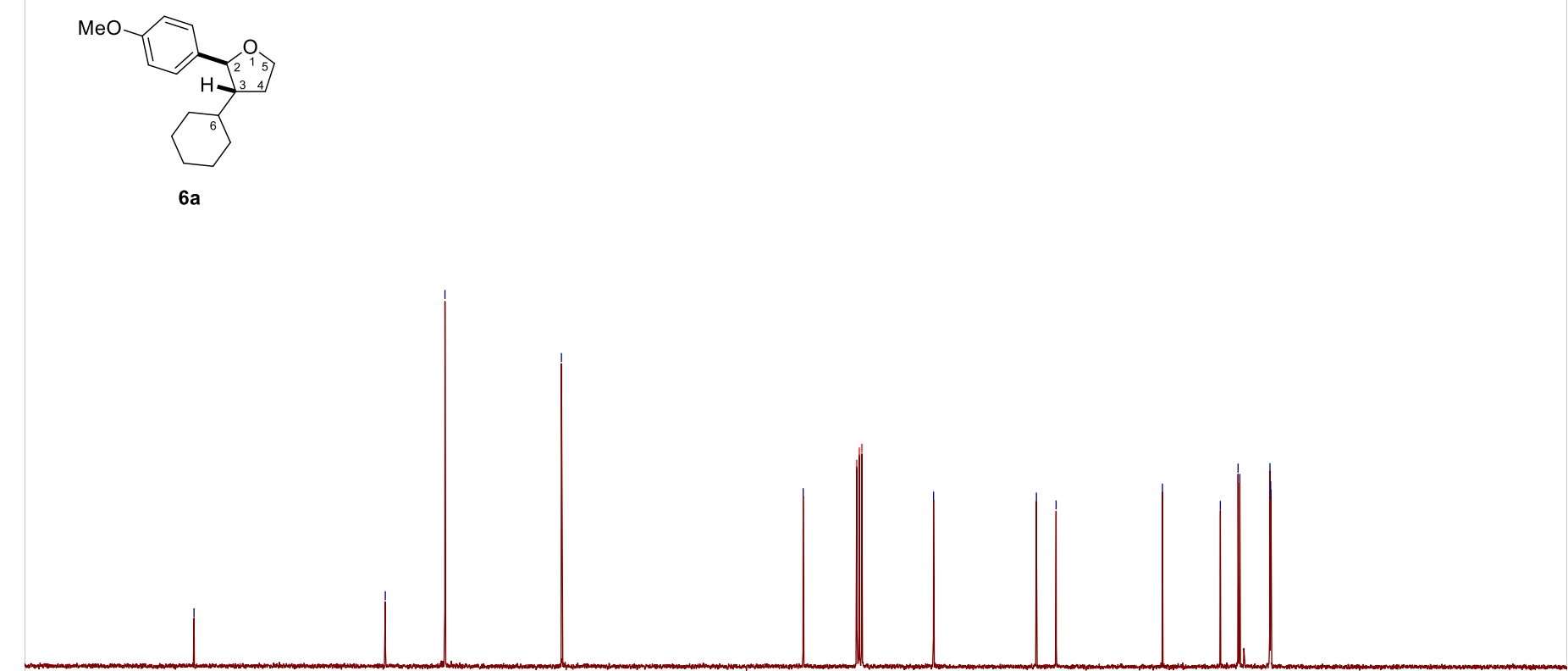

$6 a$

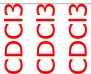

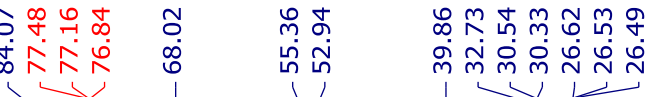


రิ

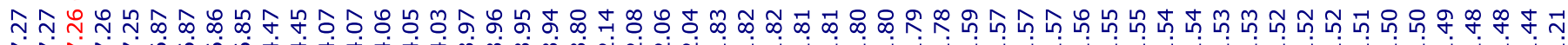
ヘ

${ }^{1} \mathrm{H}$ NMR (400 MHz, $\mathrm{CDCl}_{3}$ )

(1)

6b
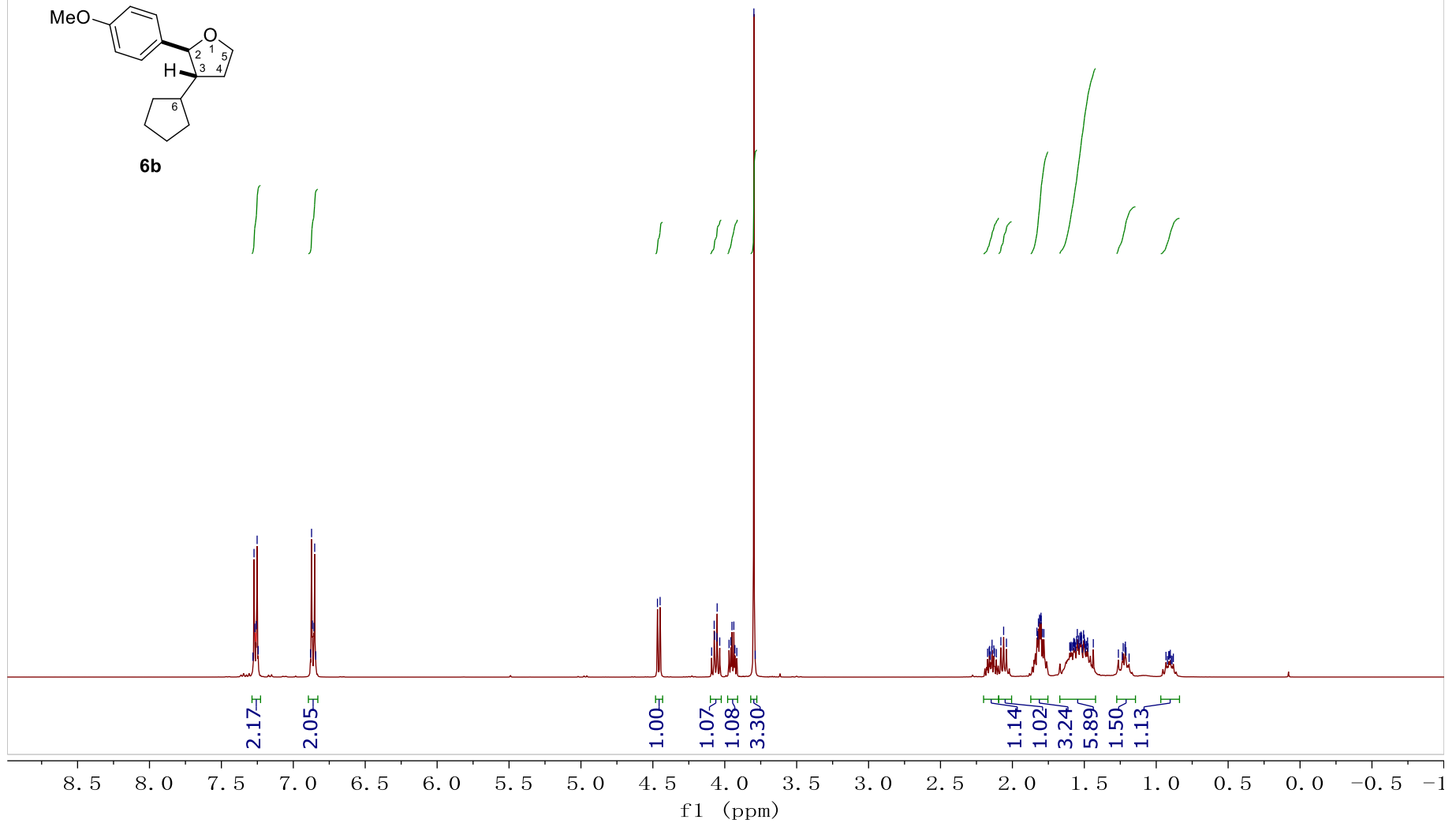

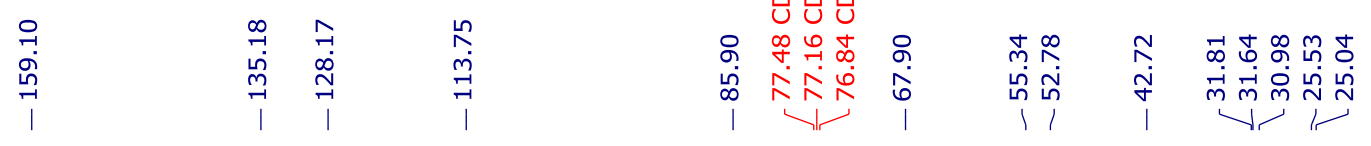

${ }^{13} \mathrm{C}$ NMR (100 MHz, $\mathrm{CDCl}_{3}$ )

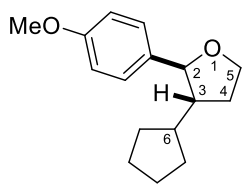

$6 b$
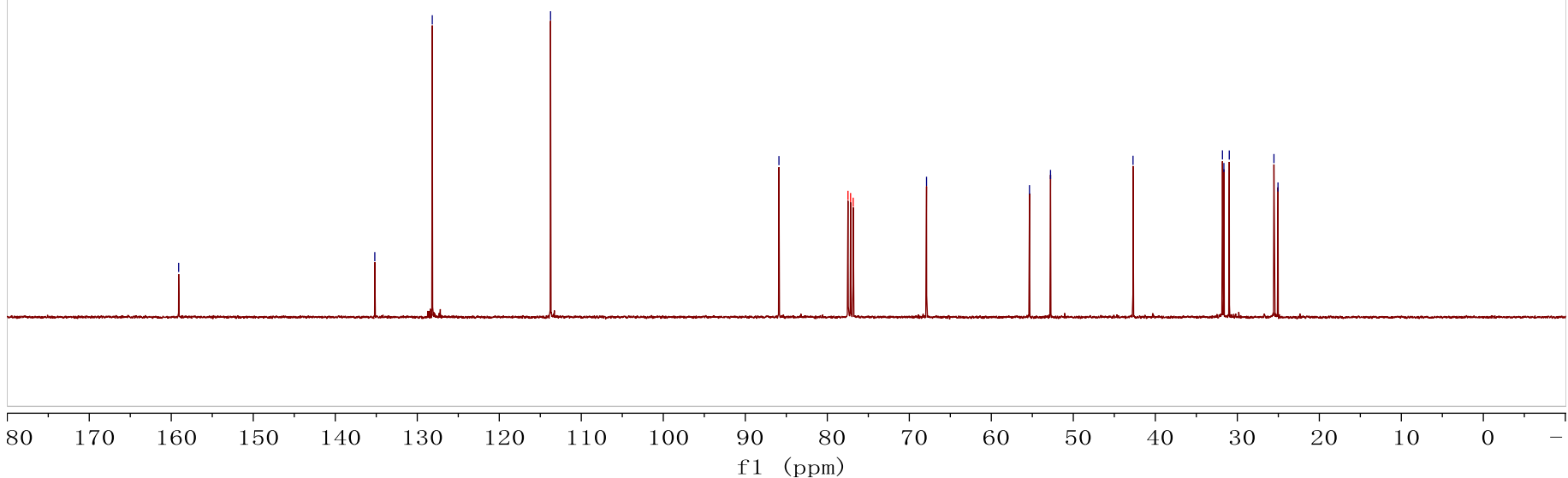
${ }^{1} \mathrm{H}$ NMR (500 MHz, $\mathrm{CDCl}_{3}$ )

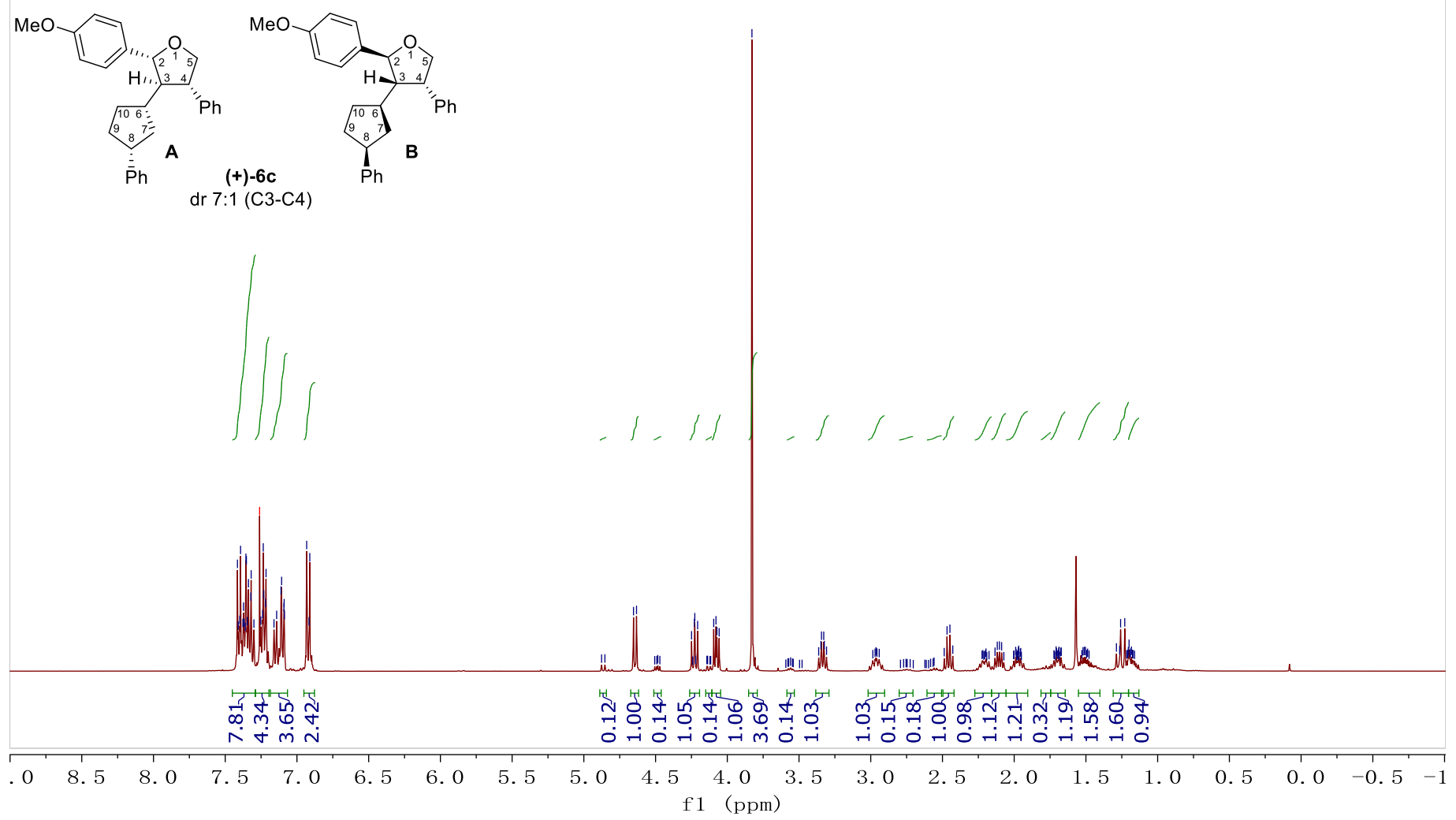

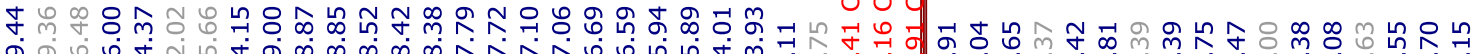

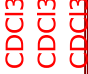

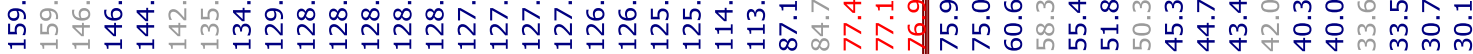
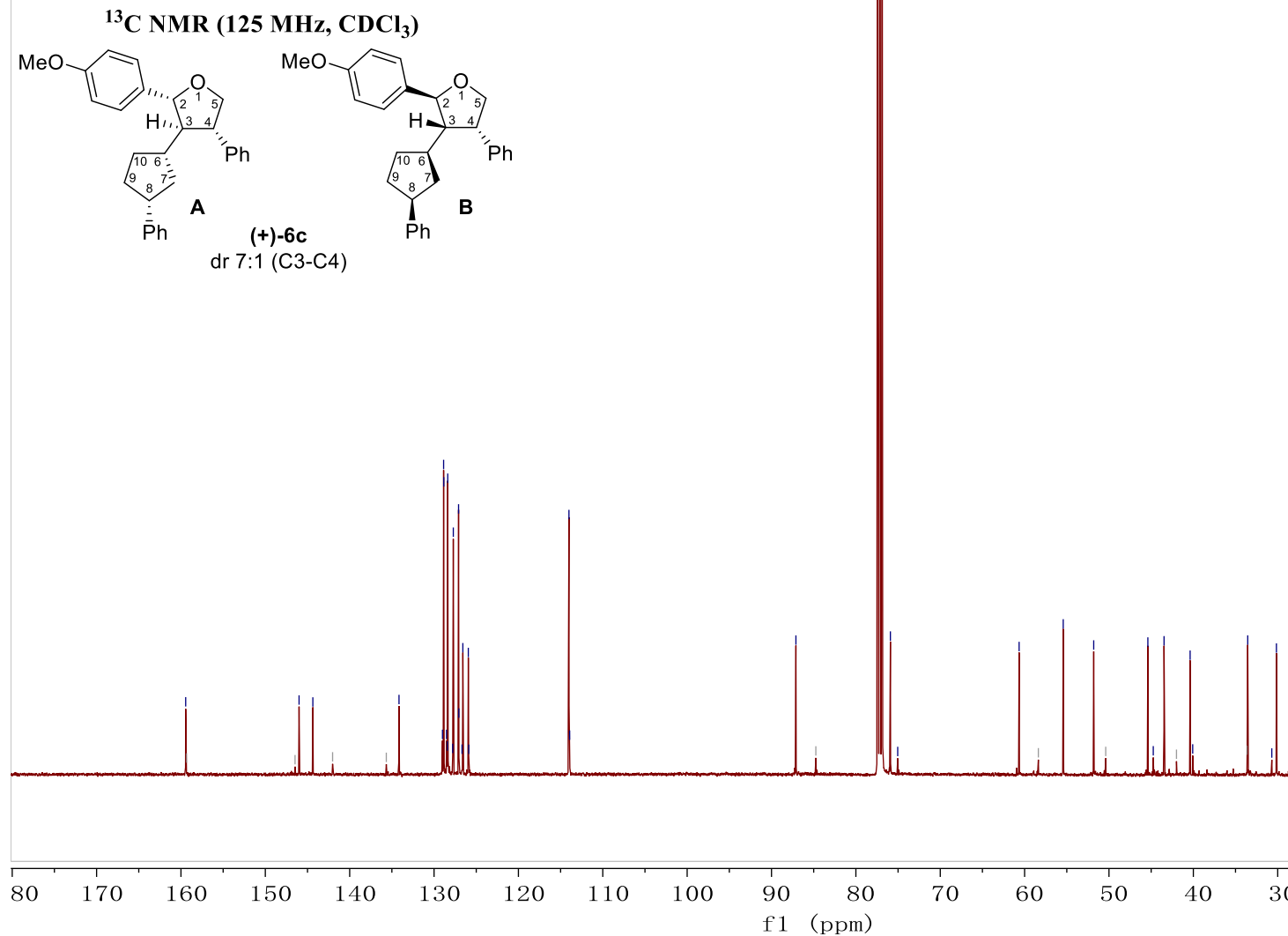
${ }^{1} \mathrm{H}-{ }^{1} \mathrm{H}$ COSY - $90\left(500 \mathrm{MHz}, \mathrm{CDCl}_{3}\right)$

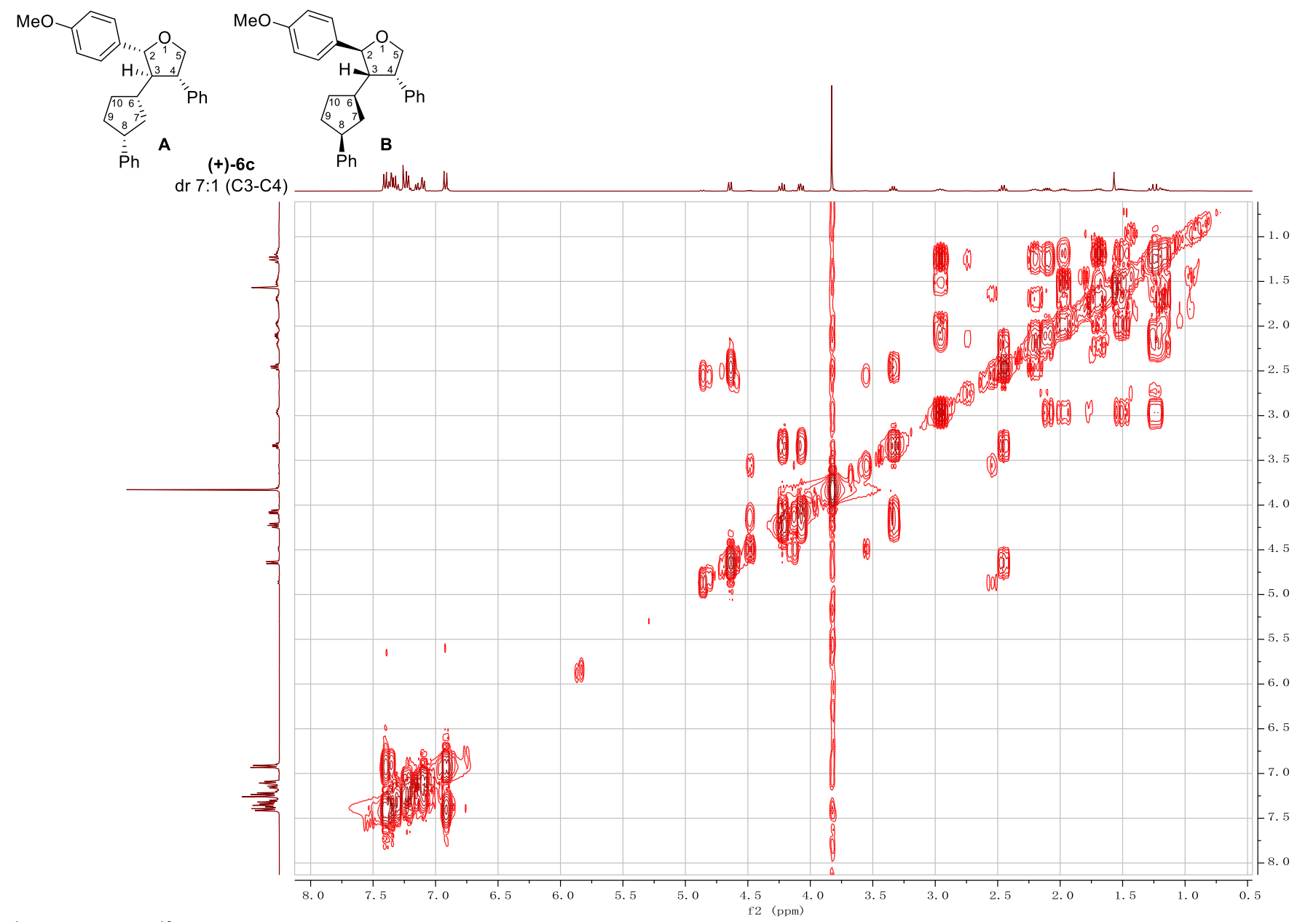

${ }^{1} \mathrm{H}(500 \mathrm{MHz})-{ }^{13} \mathrm{C}$ (125 MHz) HSQC (CDCl $)$

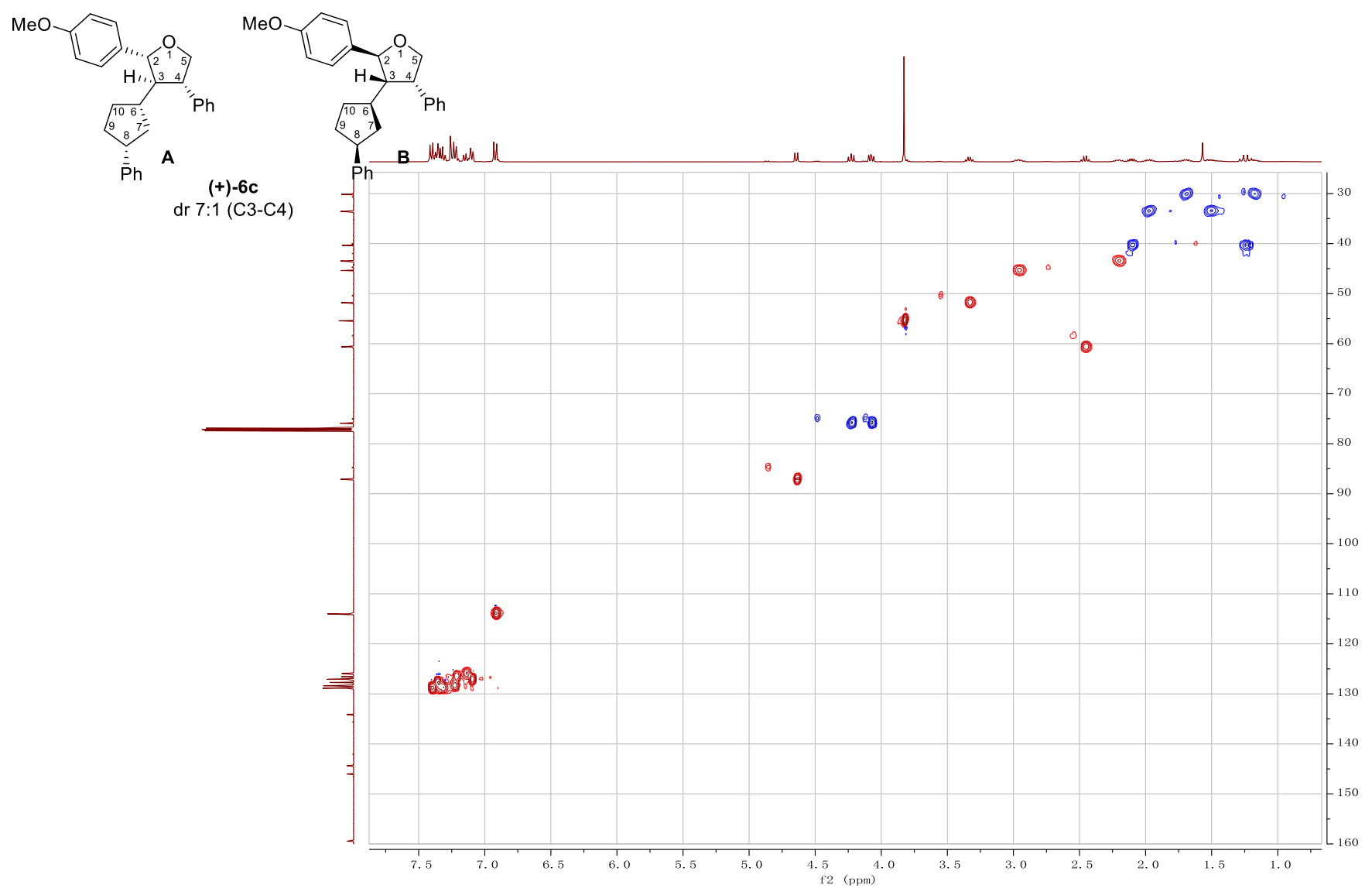


NOESY-2D (500 $\left.\mathrm{MHz}, \mathrm{CDCl}_{3}\right)$

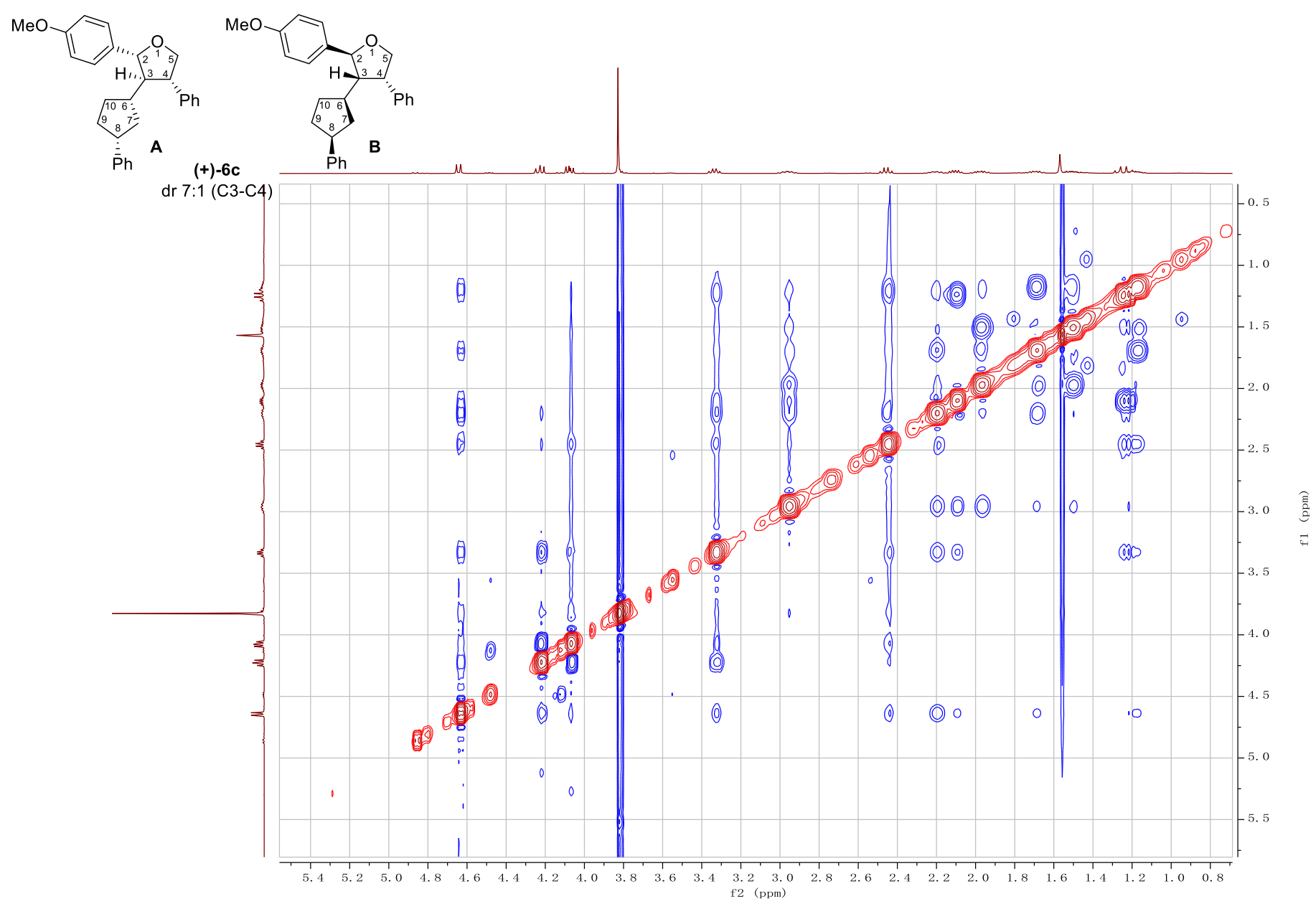


${ }^{1} \mathrm{H}$ NMR (400 MHz, $\left.\mathrm{CDCl}_{3}\right)$

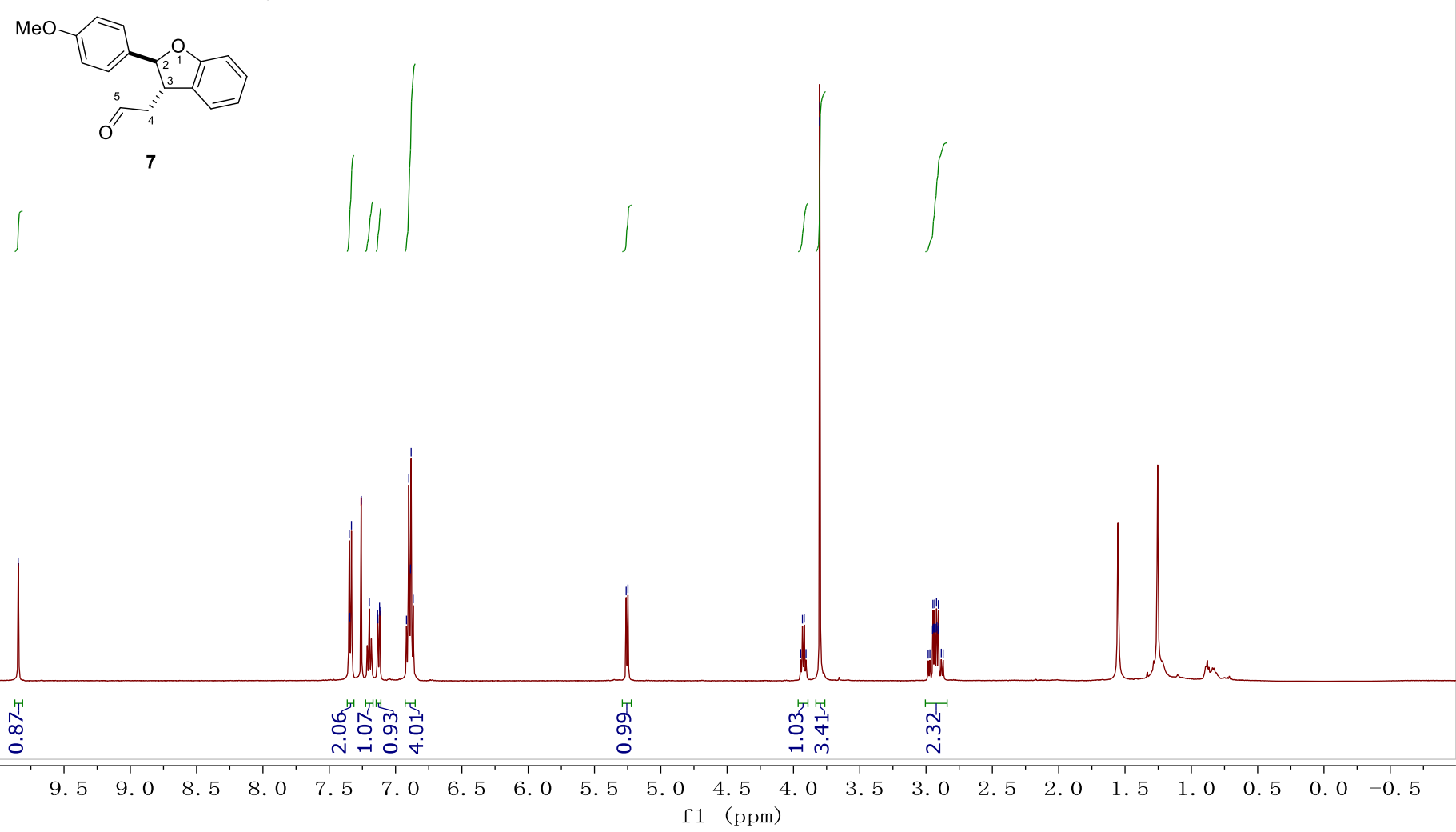

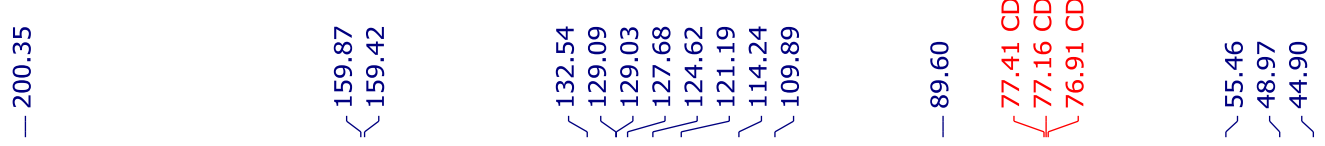

\section{${ }^{13} \mathrm{C}$ NMR (100 MHz, $\left.\mathrm{CDCl}_{3}\right)$}

$\mathrm{MeO}$<smiles>O=CC[C@H]1c2ccccc2OC1c1ccccc1</smiles>

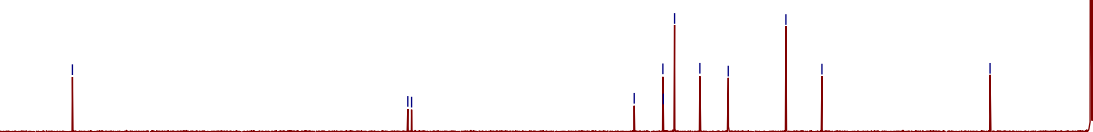

$10 \quad 20$

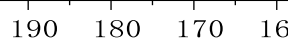

$60 \quad 150 \quad 140 \quad 130$

$120 \quad 110$ 


\section{${ }^{1} \mathrm{H}$ NMR (500 MHz, $\mathrm{CDCl}_{3}$ )}

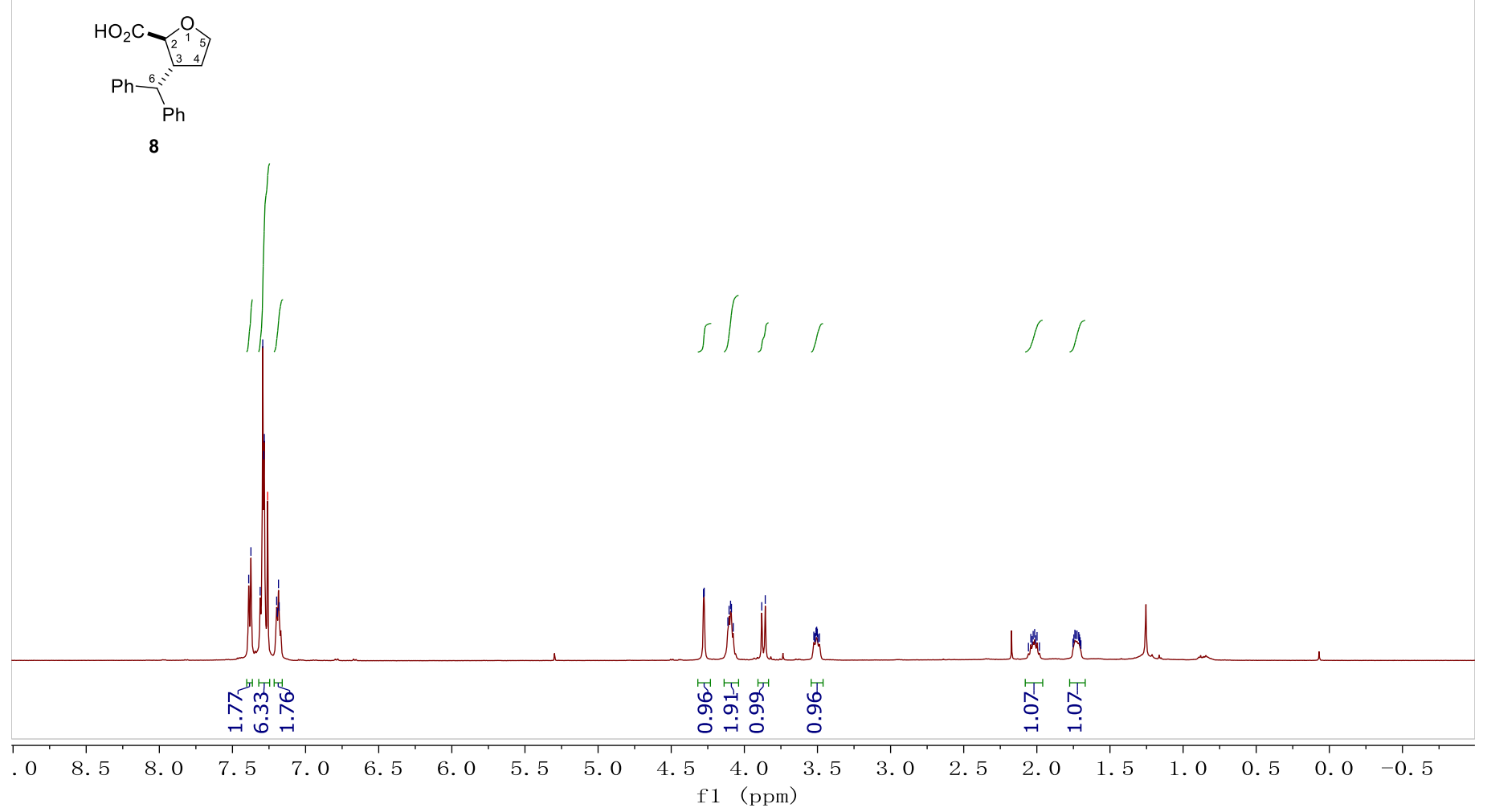

\section{g) 요 $\quad$ 요 \\ 卓 $\quad$ 垈 \\ I}
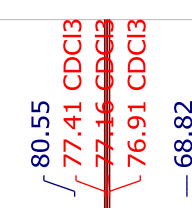

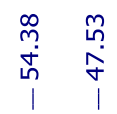

\section{กั่}

${ }^{13} \mathrm{C}$ NMR (125 MHz, $\mathrm{CDCl}_{3}$ )<smiles>O=C(O)c1occc1C(c1ccccc1)c1ccccc1</smiles>
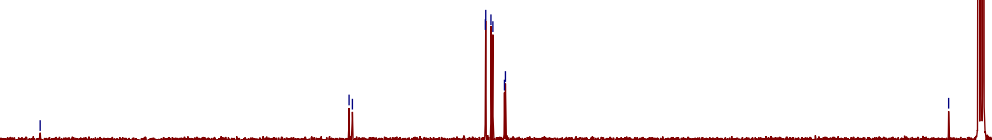

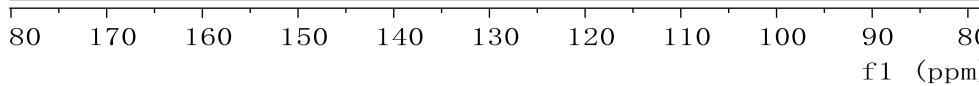


${ }^{1} \mathrm{H}$ - ${ }^{1} \mathrm{H}$ COSY - $90\left(500 \mathrm{MHz}, \mathrm{CDCl}_{3}\right)$

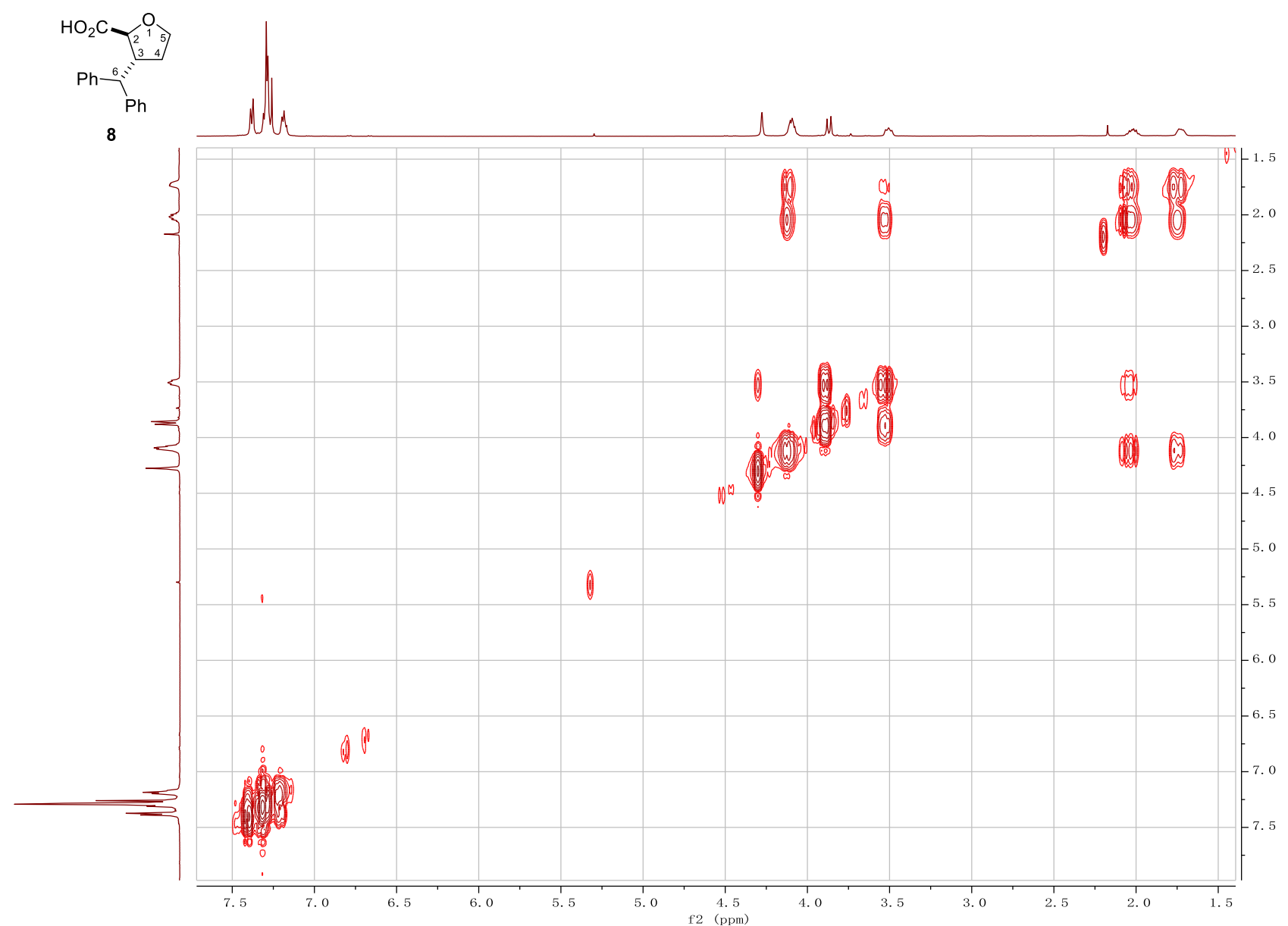

${ }^{1} \mathrm{H}(500 \mathrm{MHz})-{ }^{13} \mathrm{C}$ (125 MHz) HSQC $\left(\mathrm{CDCl}_{3}\right)$

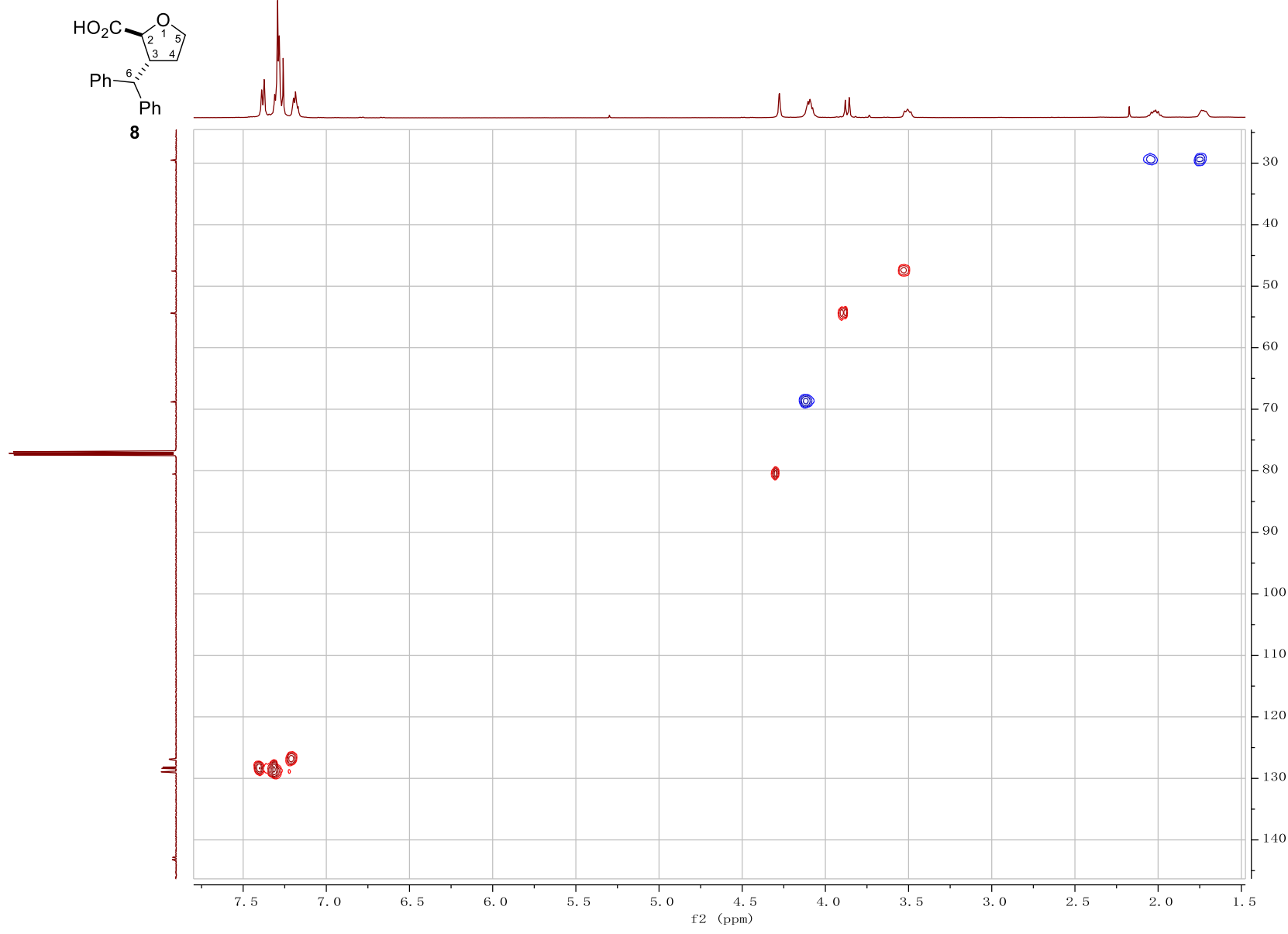




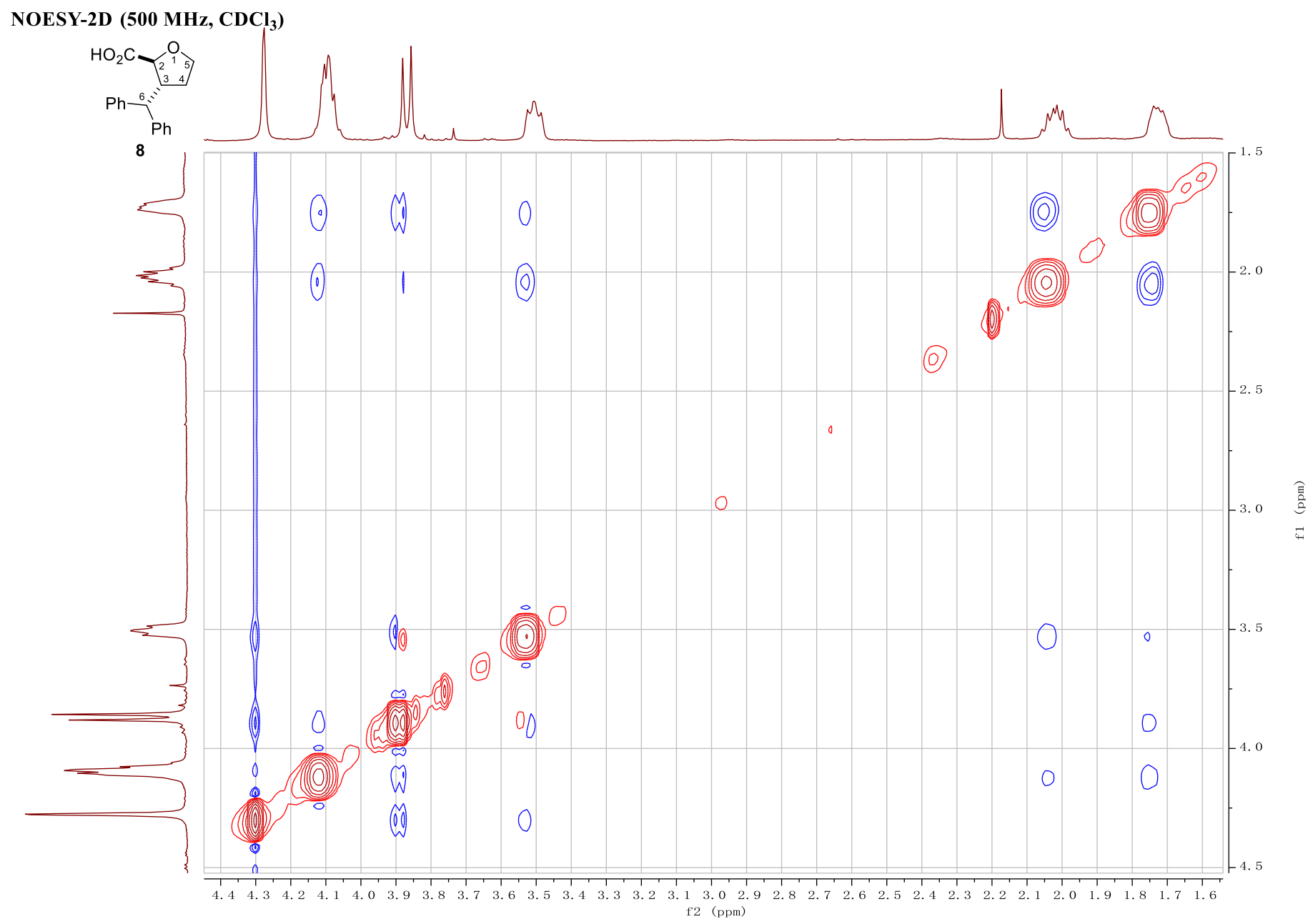


${ }^{1} \mathrm{H}$ NMR (400 MHz, $\left.\mathrm{CDCl}_{3}\right)$

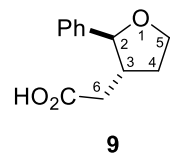
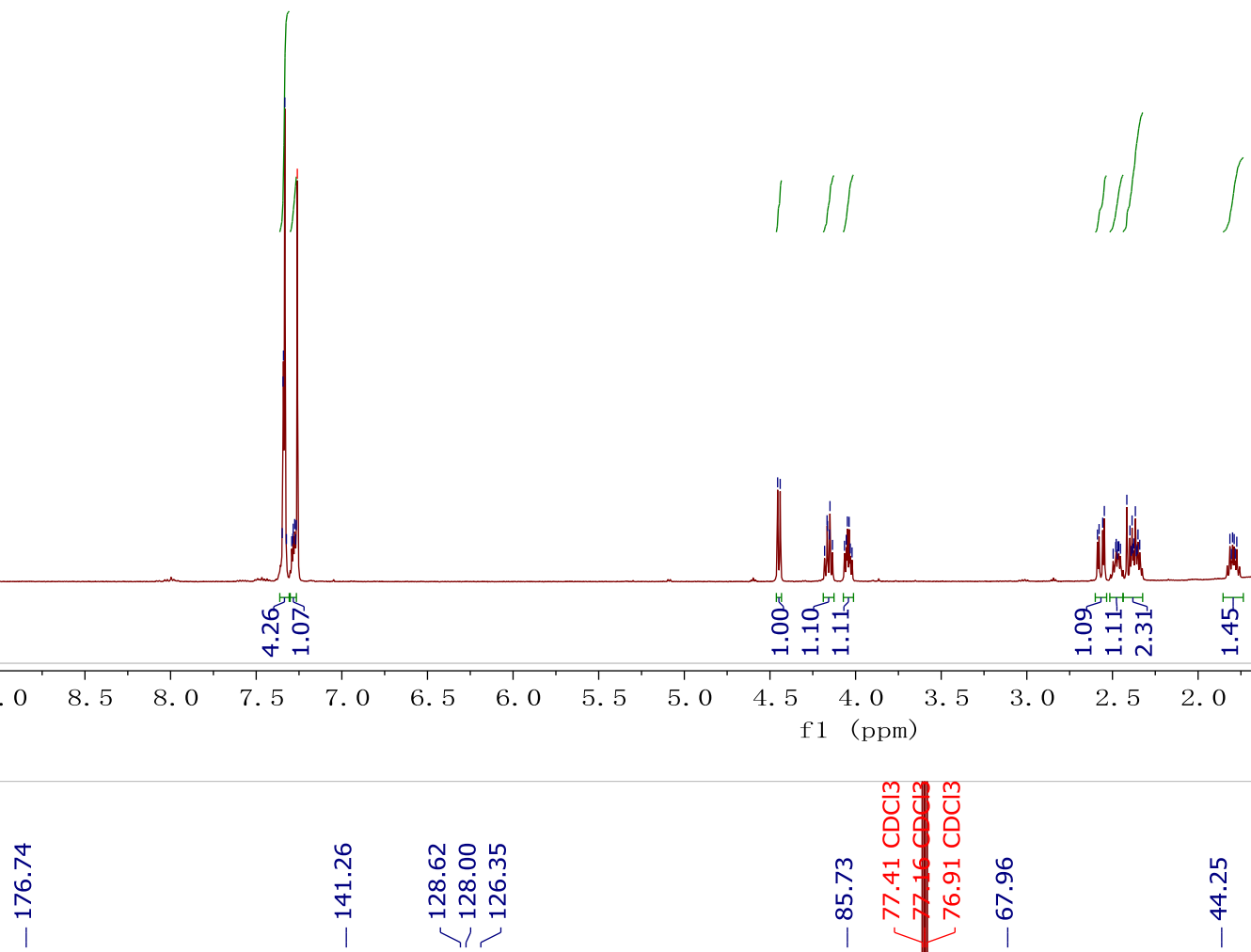

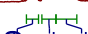

엄 ने

苗

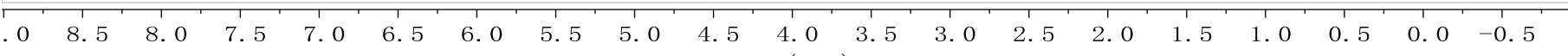
f1 (ppm)

${ }^{13} \mathrm{C}$ NMR (100 MHz, $\left.\mathrm{CDCl}_{3}\right)$
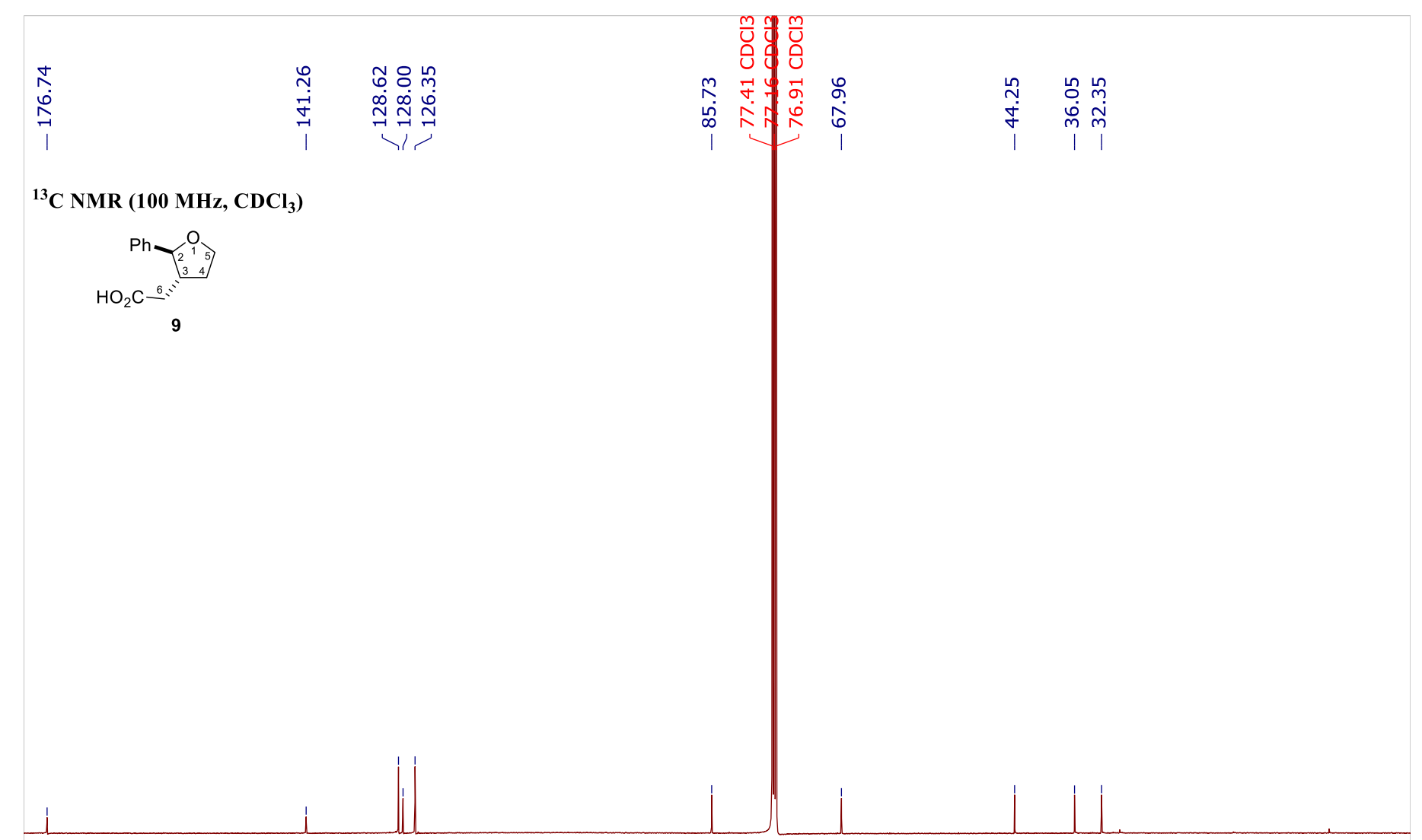


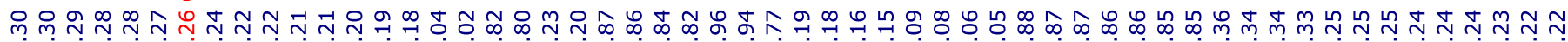

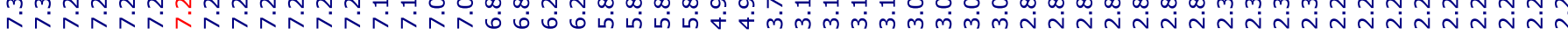

${ }^{1} \mathrm{H}$ NMR (500 MHz, $\left.\mathrm{CDCl}_{3}\right)$
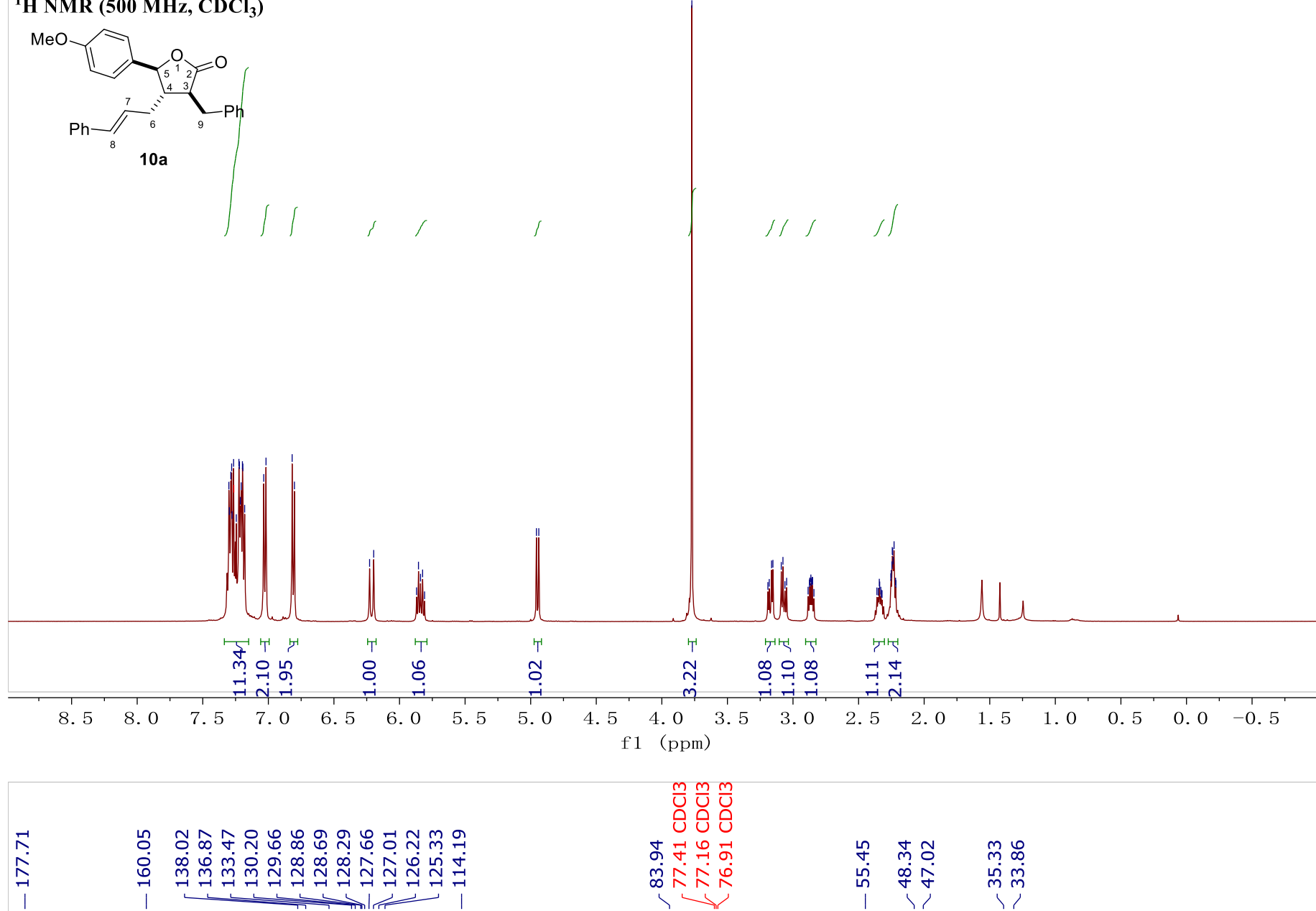

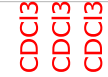

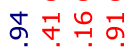

๓்ド゚

ฆ)

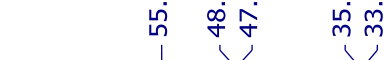

\section{${ }^{13} \mathrm{C}$ NMR (125 MHz, $\left.\mathrm{CDCl}_{3}\right)$}
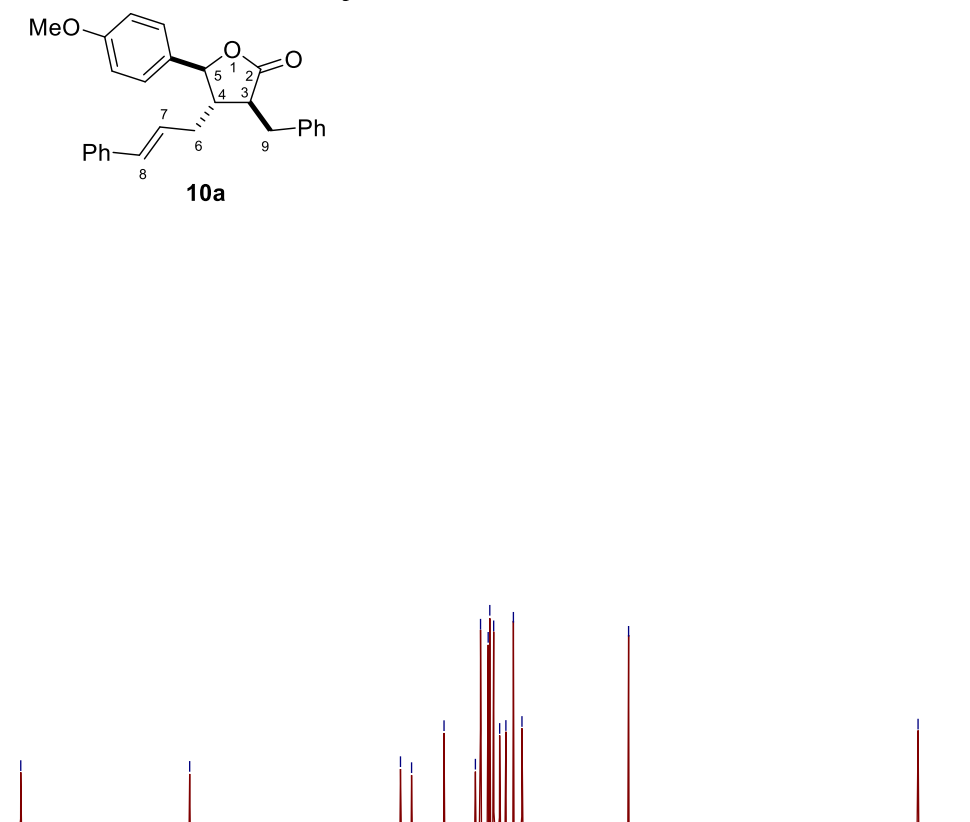

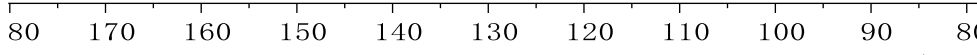


${ }^{1} \mathrm{H}$ - ${ }^{1} \mathrm{H}$ COSY - $90\left(500 \mathrm{MHz}, \mathrm{CDCl}_{3}\right)$

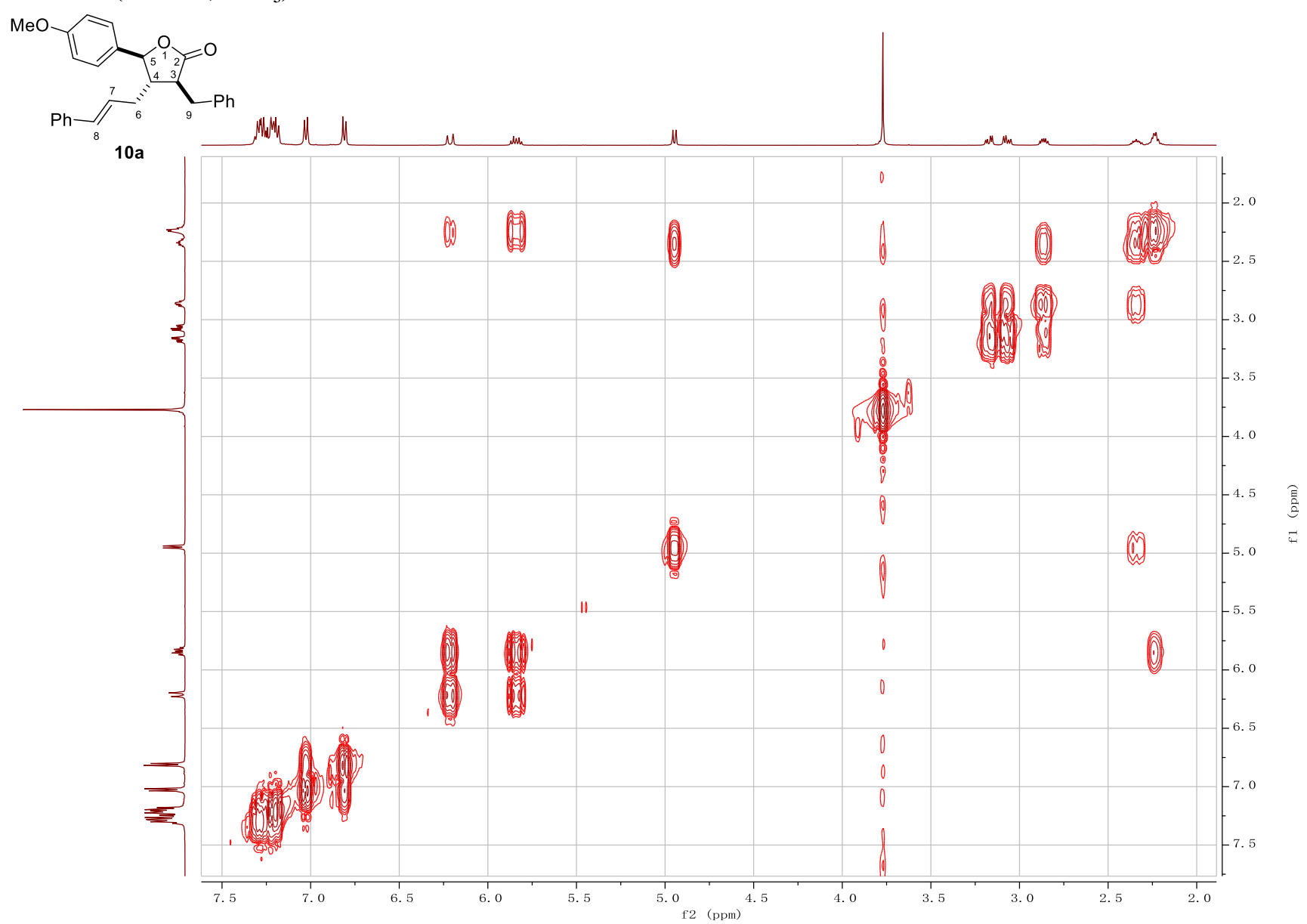

${ }^{1} \mathrm{H}(500 \mathrm{MHz})-{ }^{13} \mathrm{C}(125 \mathrm{MHz}) \mathrm{HSQC}\left(\mathrm{CDCl}_{3}\right)$

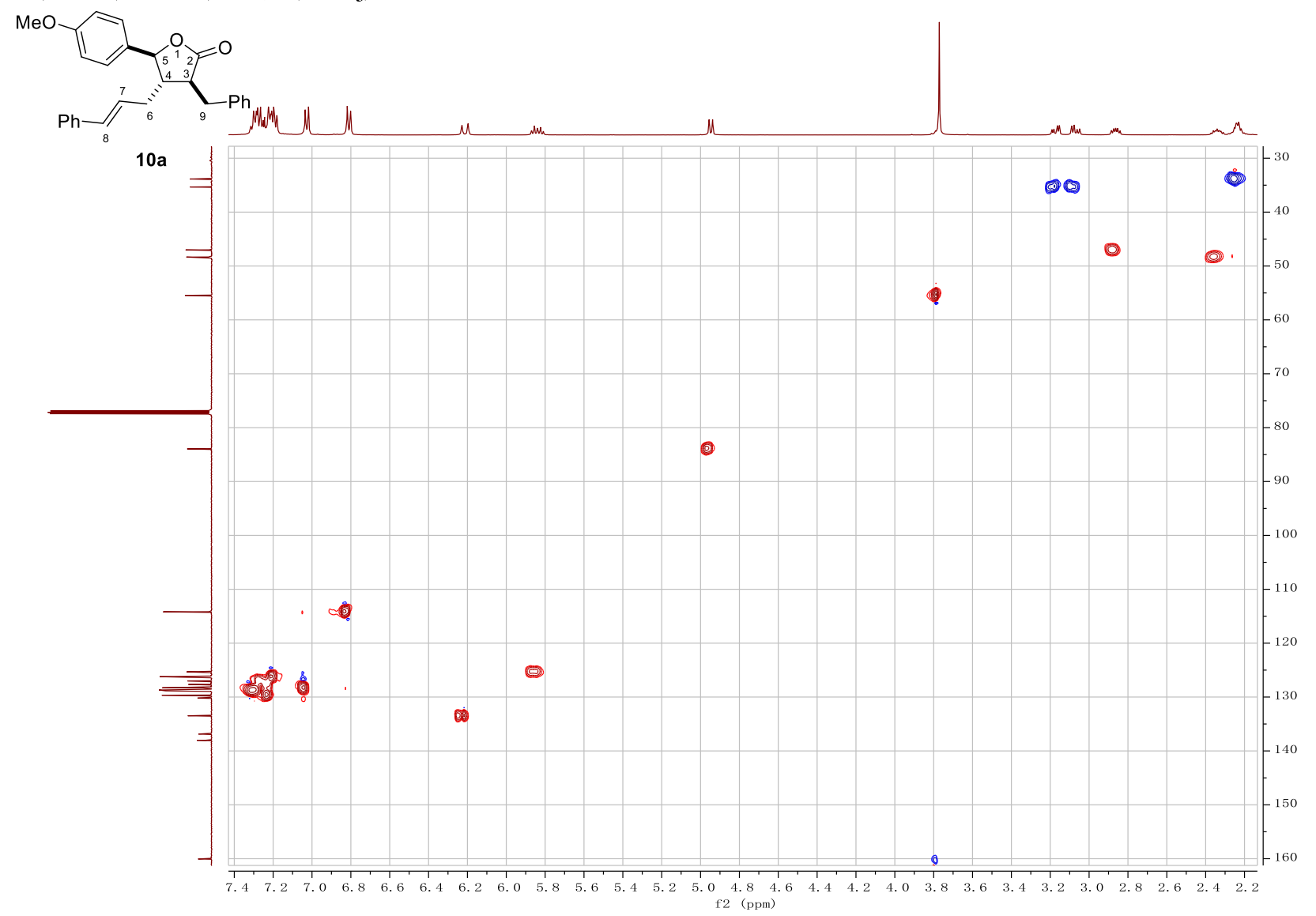


NOESY-2D (500 MHz, $\mathrm{CDCl}_{3}$ )

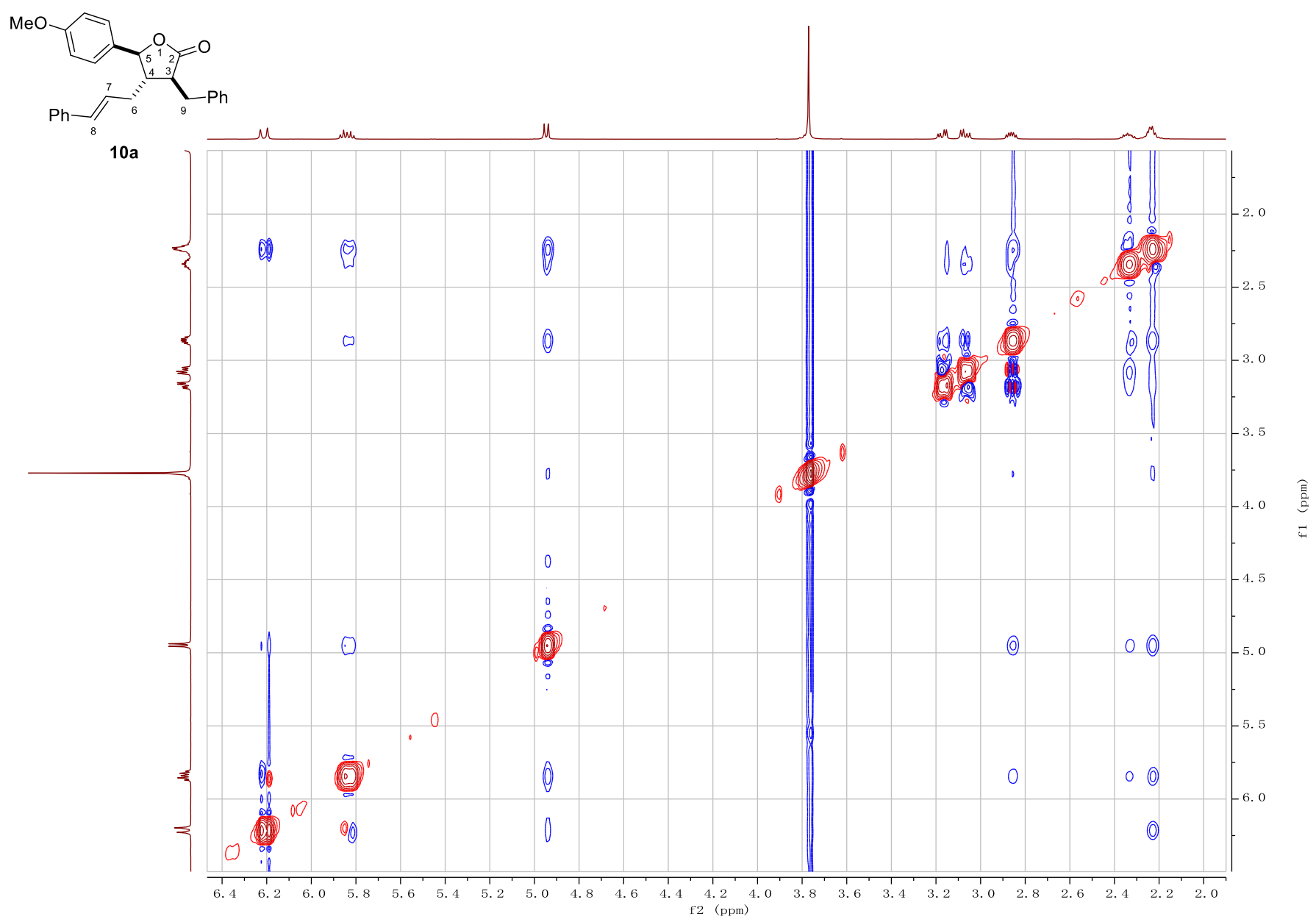




\section{${ }^{1} \mathrm{H}$ NMR (500 MHz, $\mathrm{CDCl}_{3}$ )}

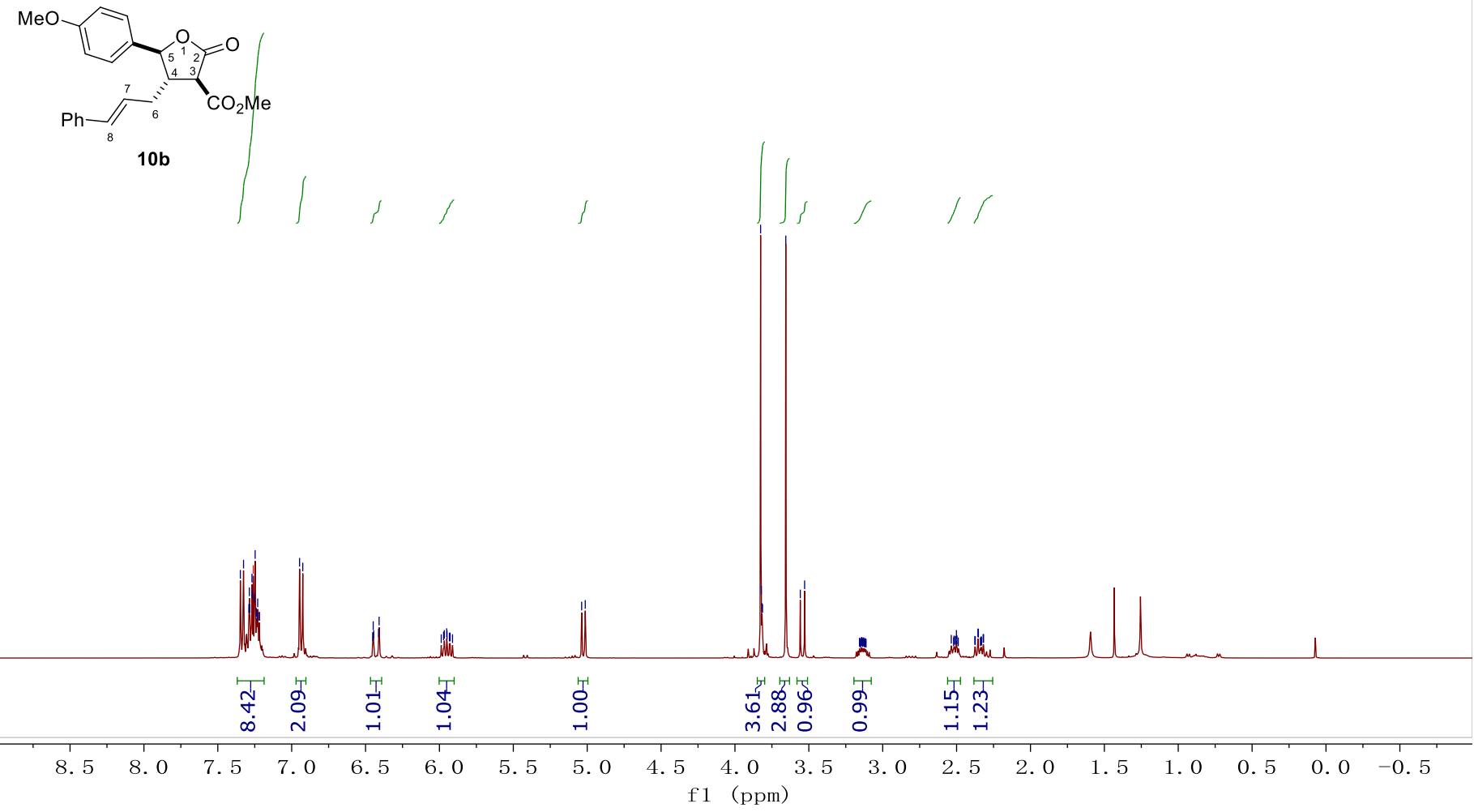

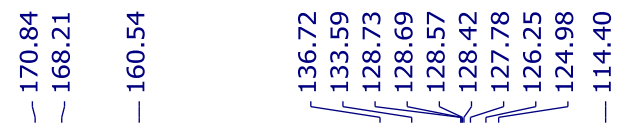

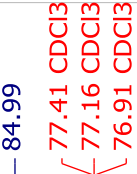

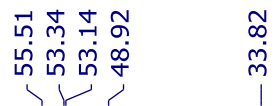

\section{${ }^{13} \mathrm{C}$ NMR (125 MHz, $\left.\mathrm{CDCl}_{3}\right)$}
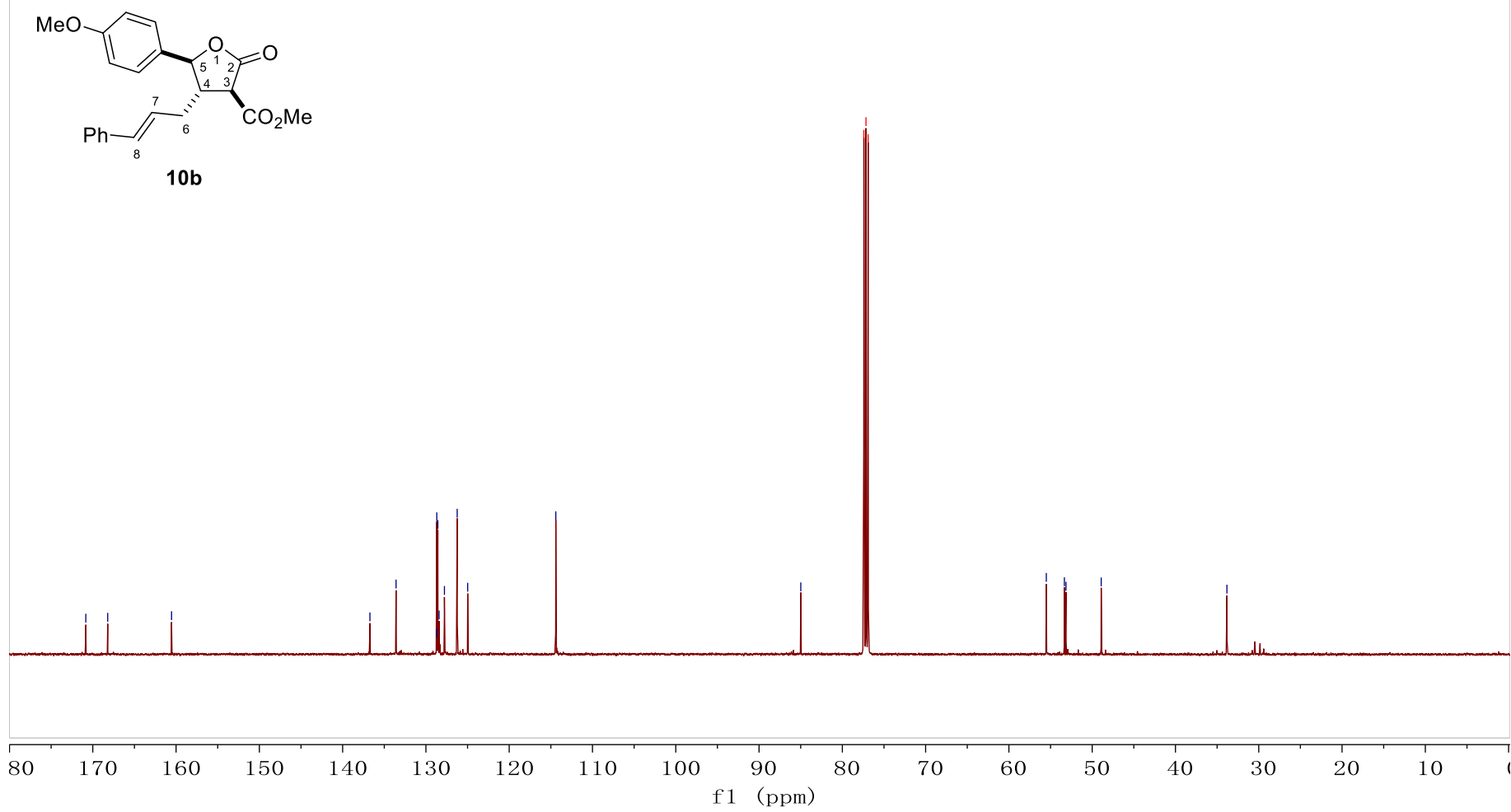
${ }^{1} \mathrm{H}-{ }^{1} \mathrm{H}$ COSY - $90\left(500 \mathrm{MHz}, \mathrm{CDCl}_{3}\right)$

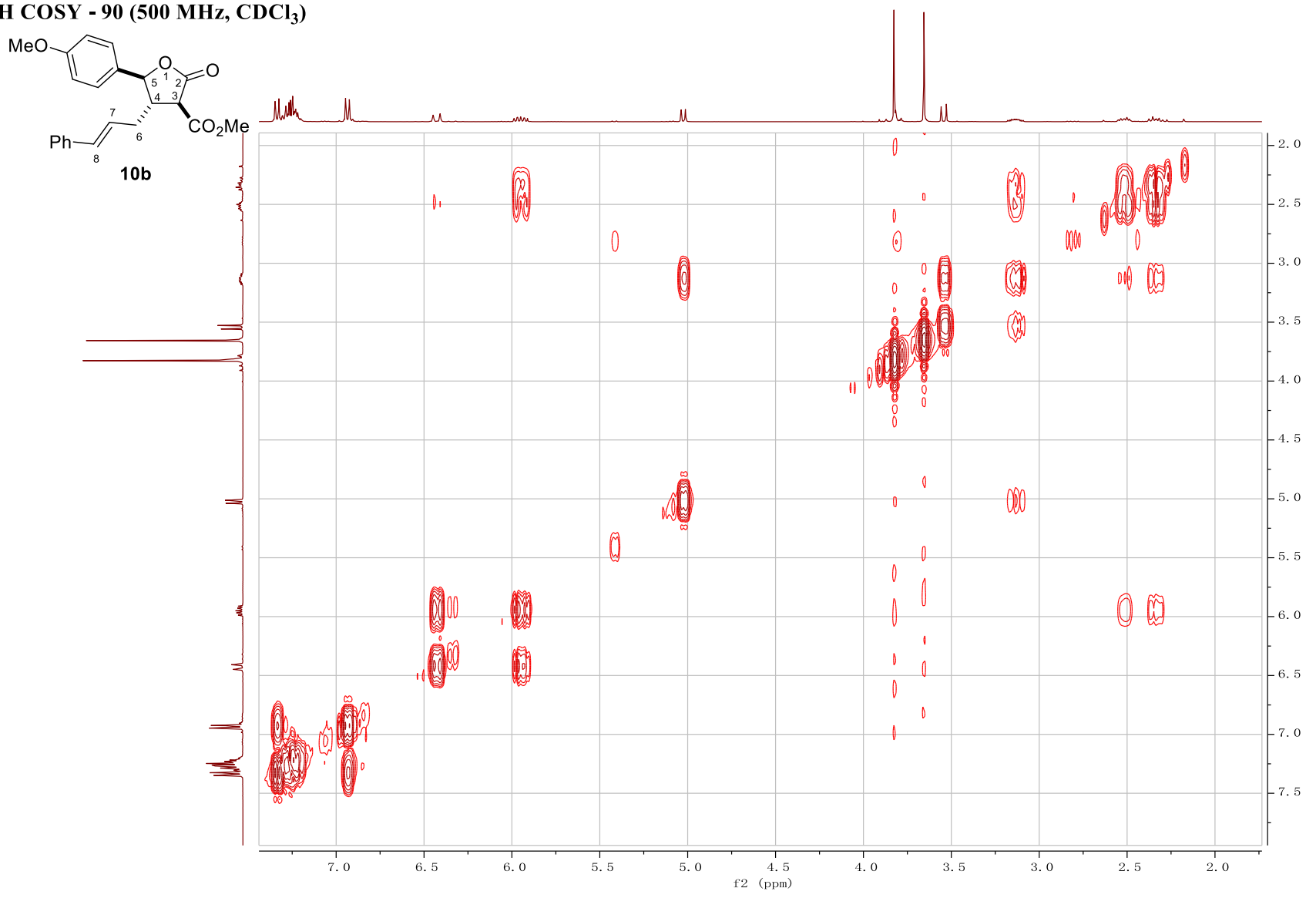

${ }^{1} \mathrm{H}(500 \mathrm{MHz})={ }^{13} \mathrm{C}$ (125 MHz) HSQC (CDCl $)$

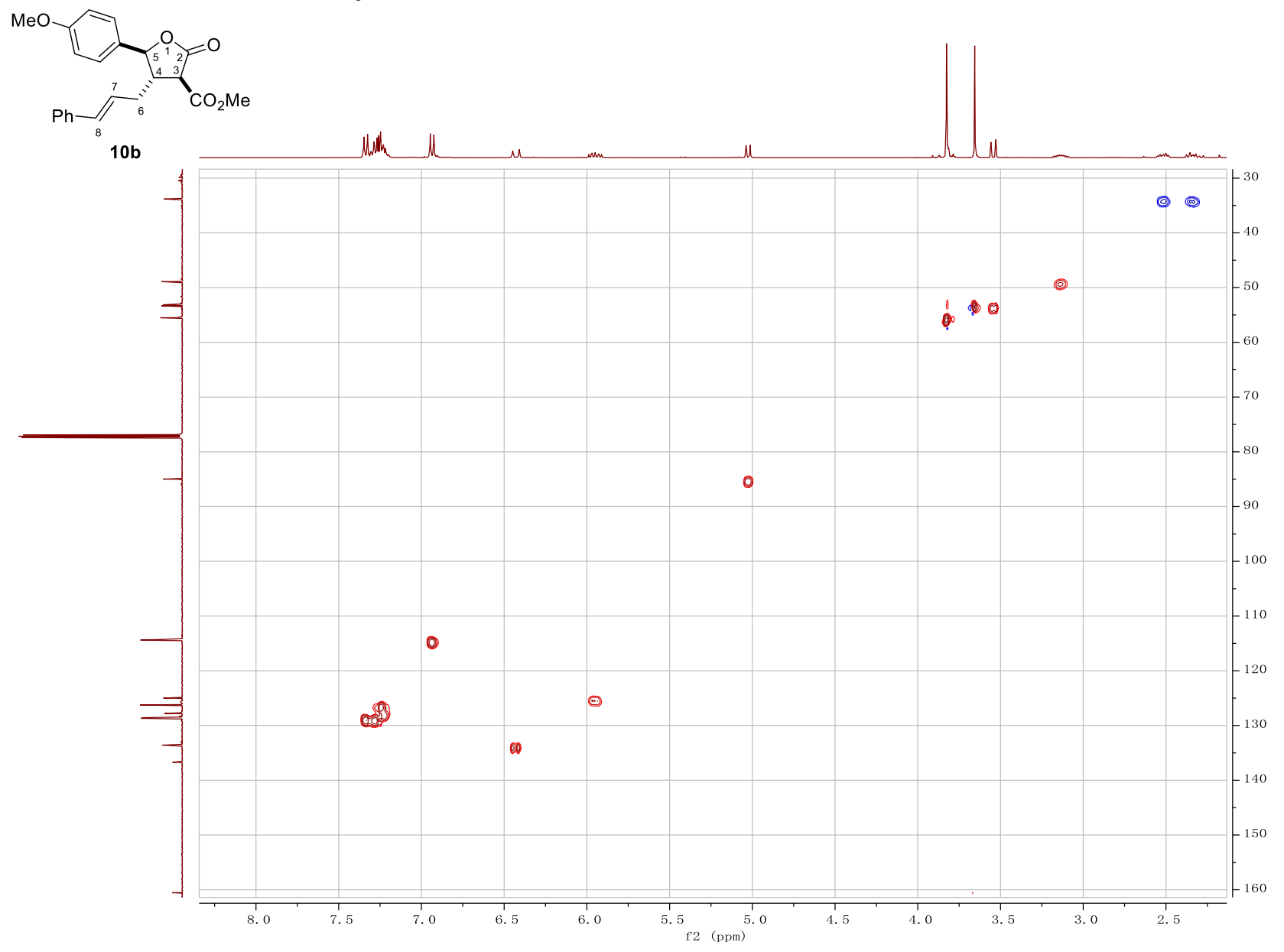




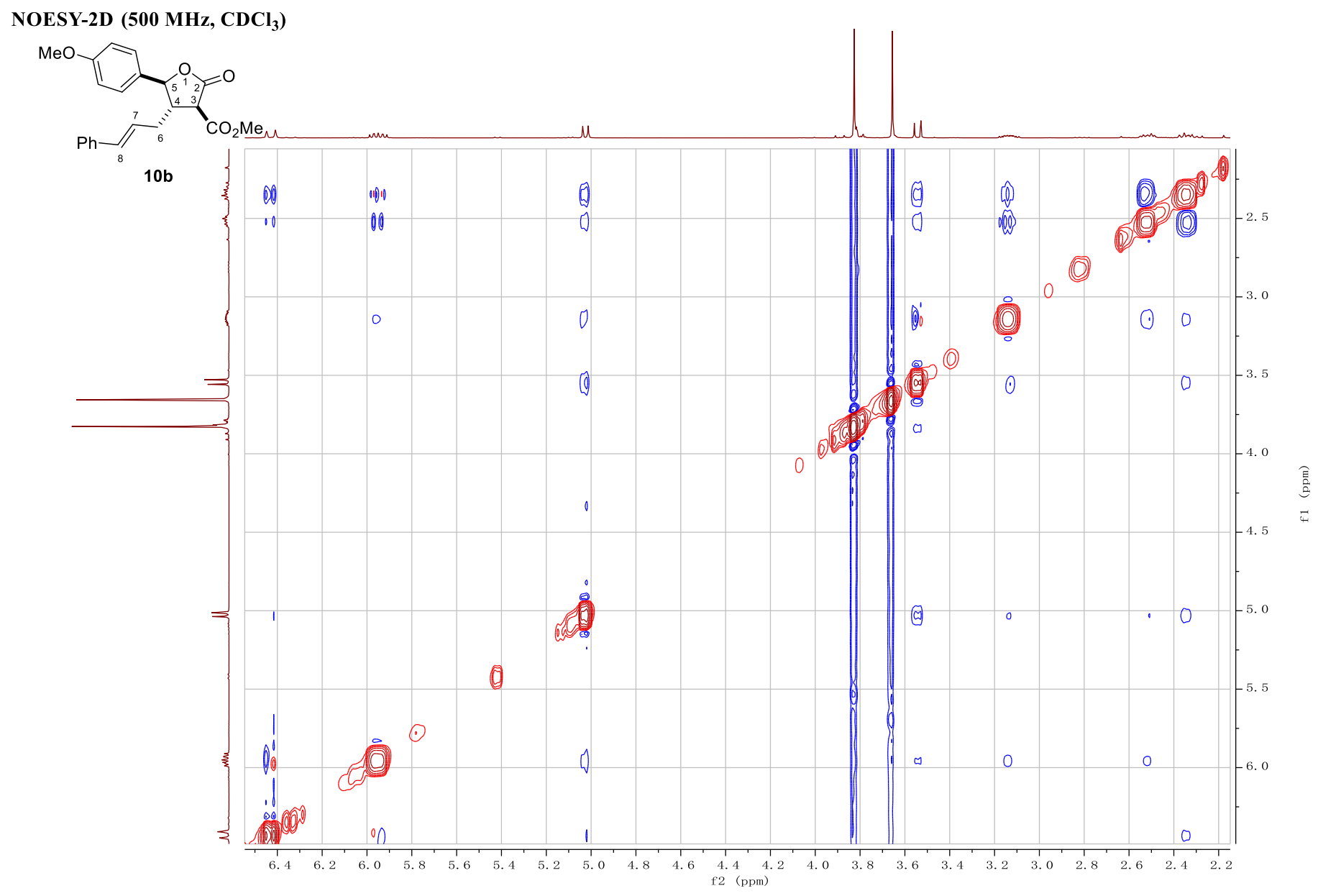

S88 


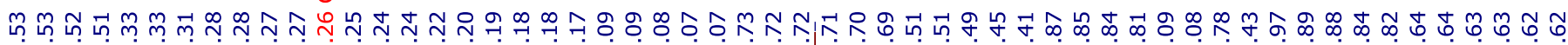

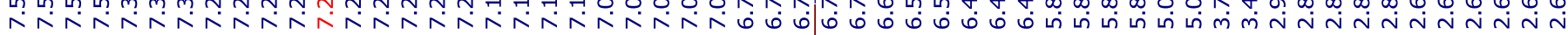

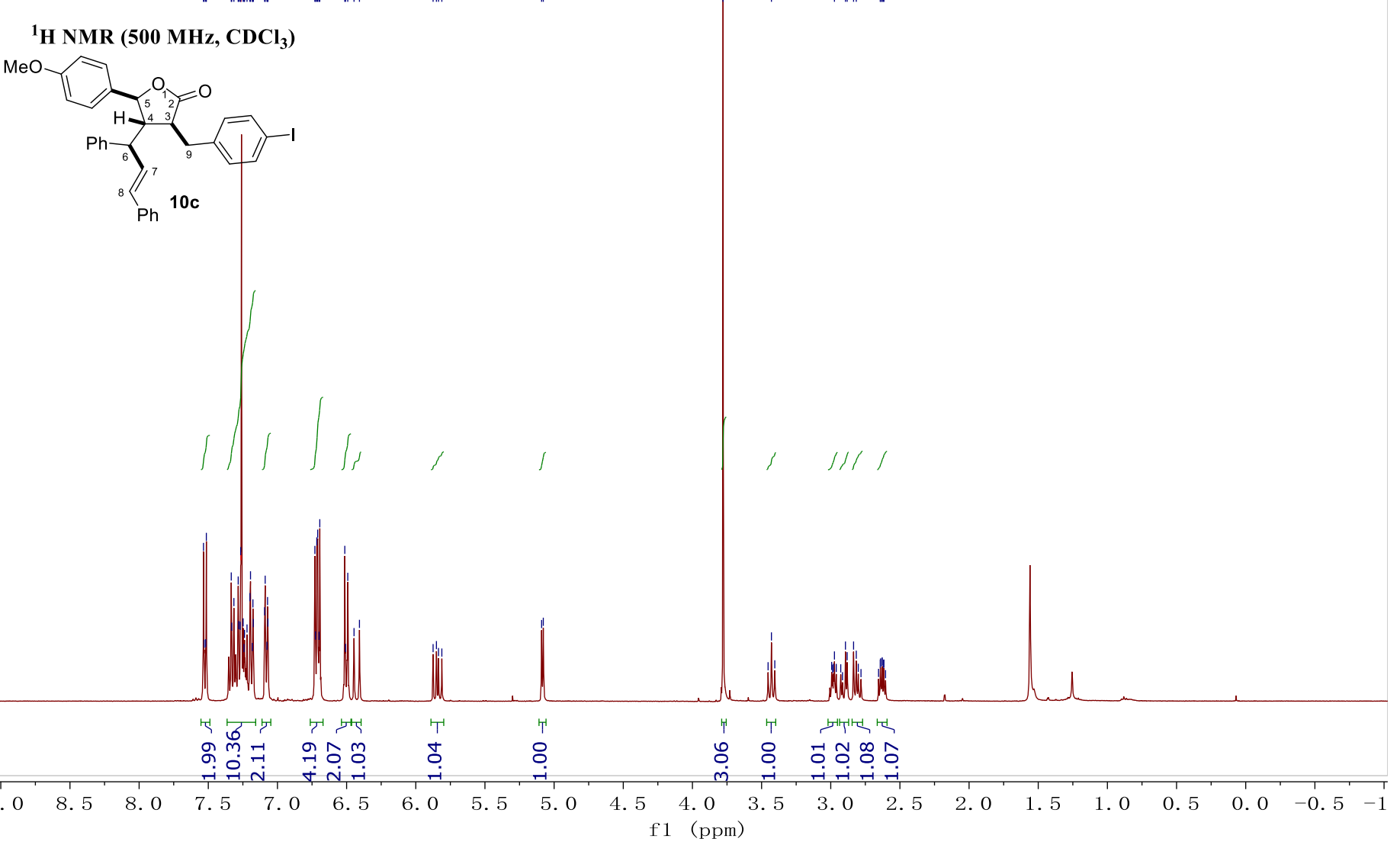

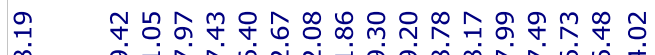

品 $\quad \underbrace{0}$

는

子.

ส

ๆ

${ }^{13} \mathrm{C}$ NMR (125 MHz, $\left.\mathrm{CDCl}_{3}\right)$

$\mathrm{MeO}$
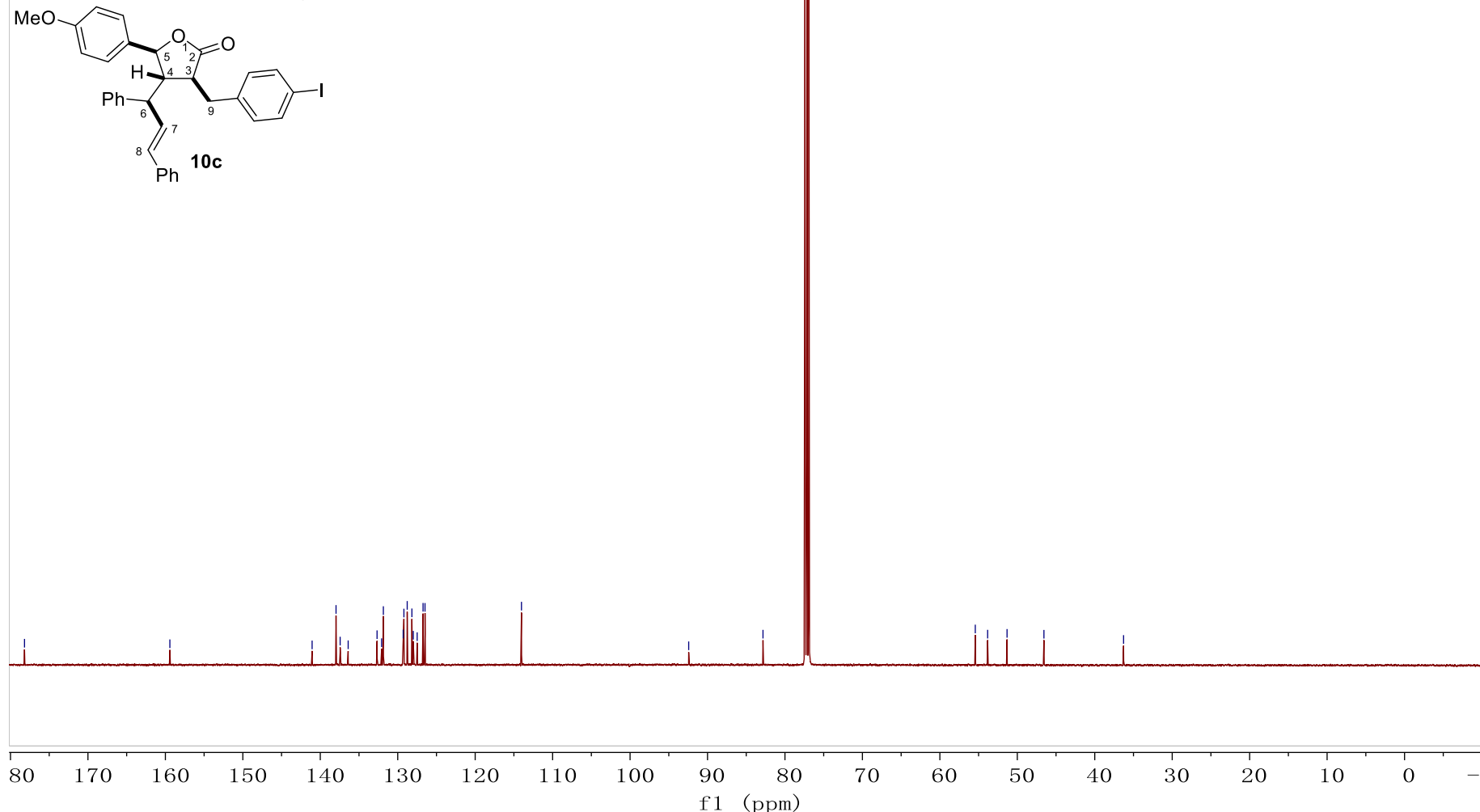

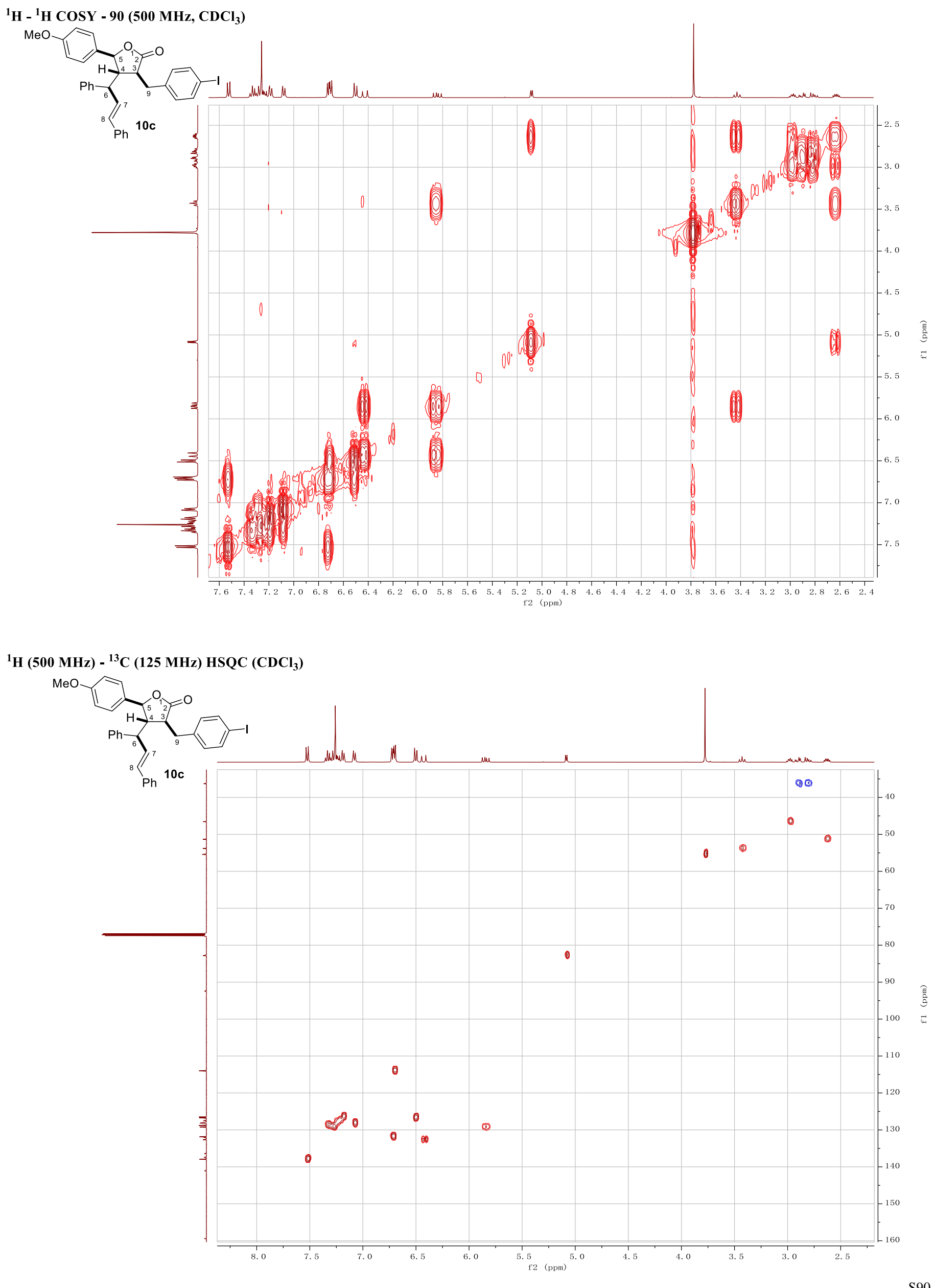
NOESY-2D (500 MHz, $\mathrm{CDCl}_{3}$ )

$\mathrm{MeO}$

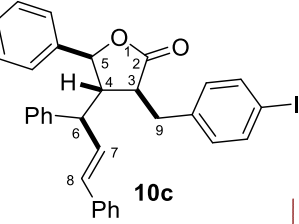

$\mathrm{Ph}$ 10c

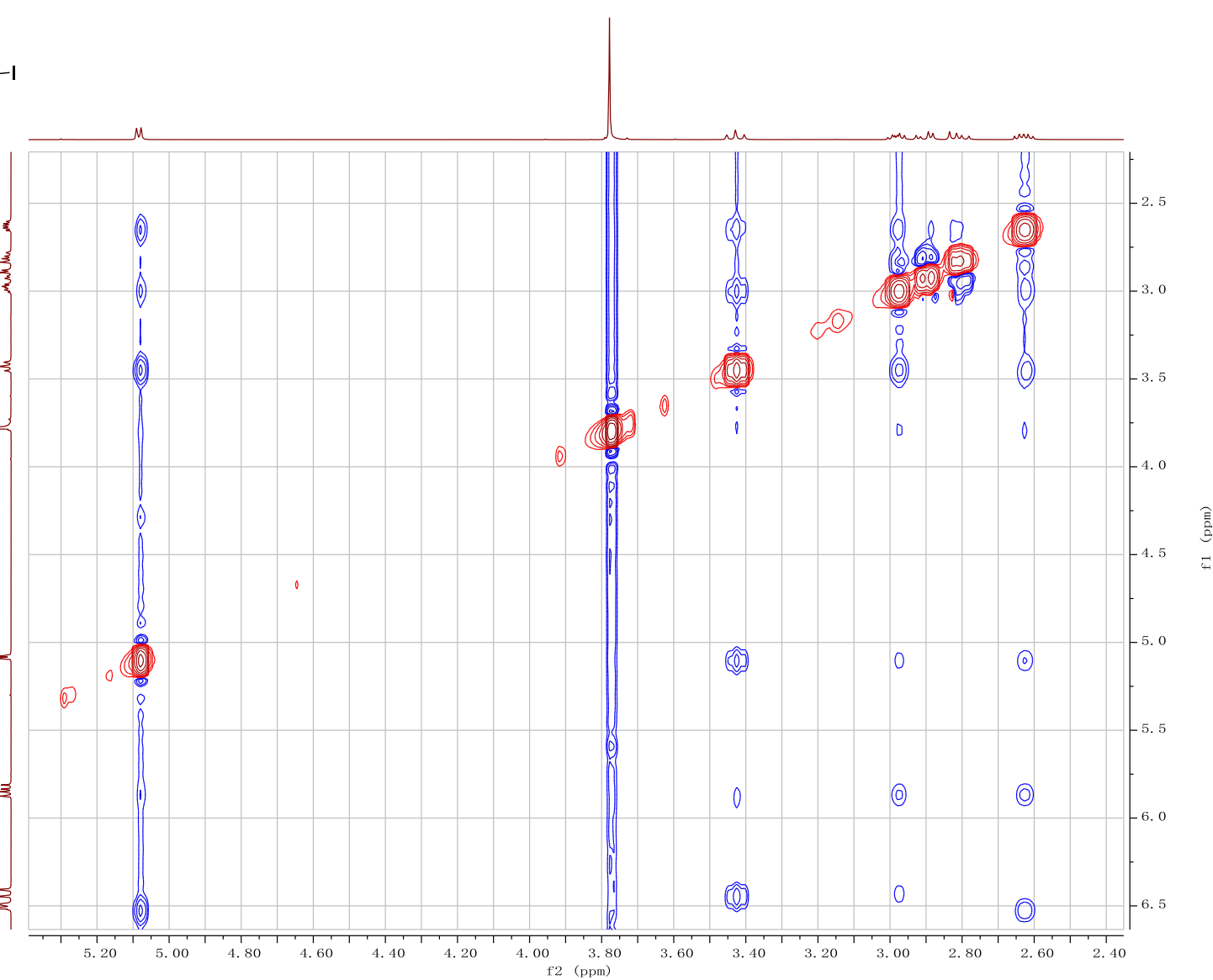




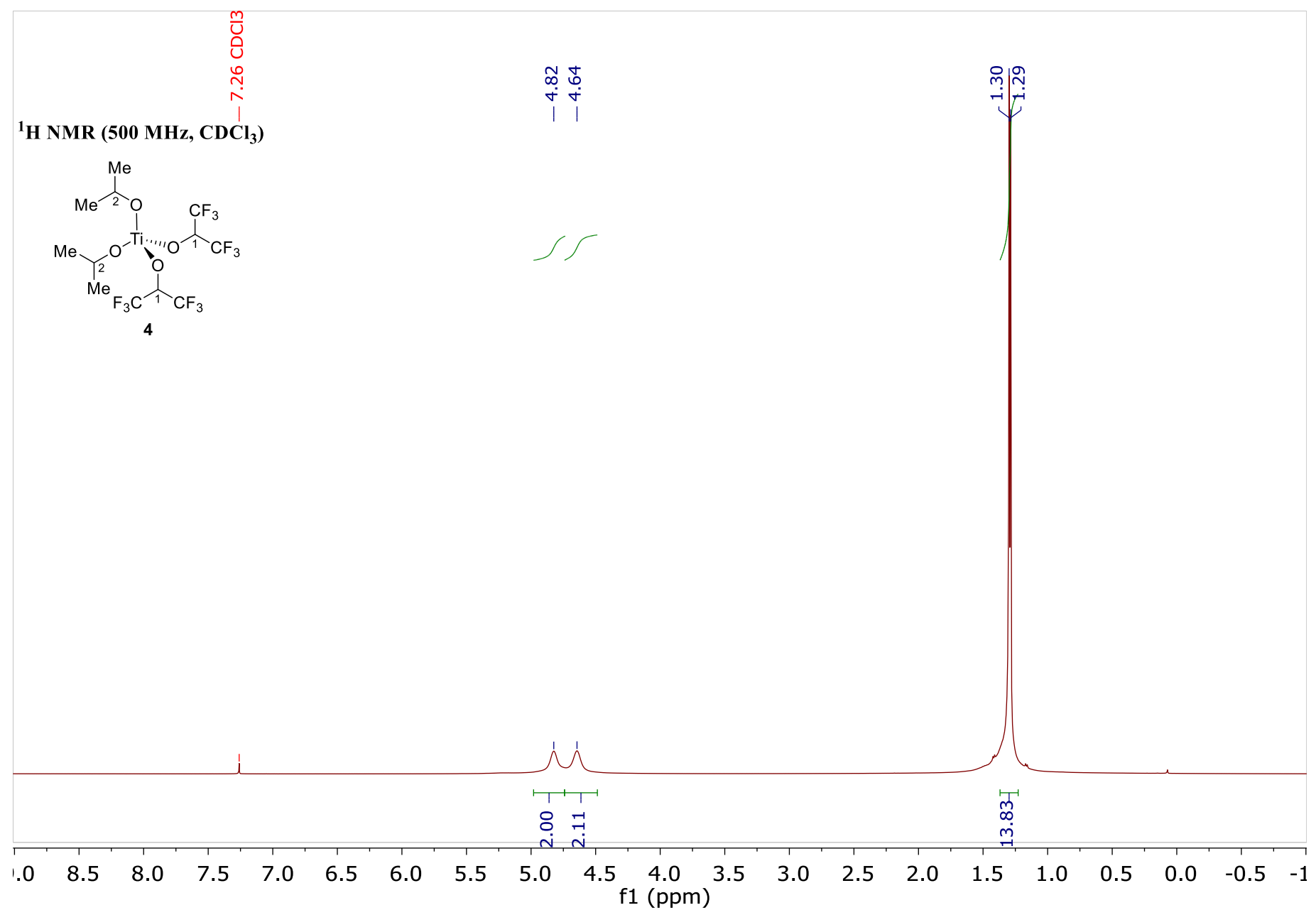

\section{${ }^{13} \mathrm{C}$ NMR (125 MHz, $\mathrm{CDCl}_{3}$ )}

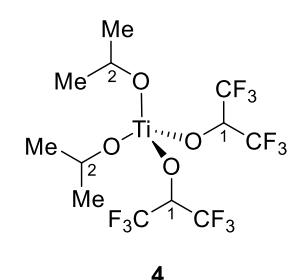

ने बे ఫั

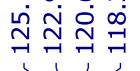

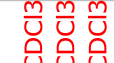

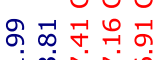

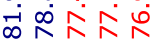

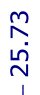


${ }^{1} \mathrm{H}-{ }^{1} \mathrm{H}$ COSY - $90\left(500 \mathrm{MHz}, \mathrm{CDCl}_{3}\right)$
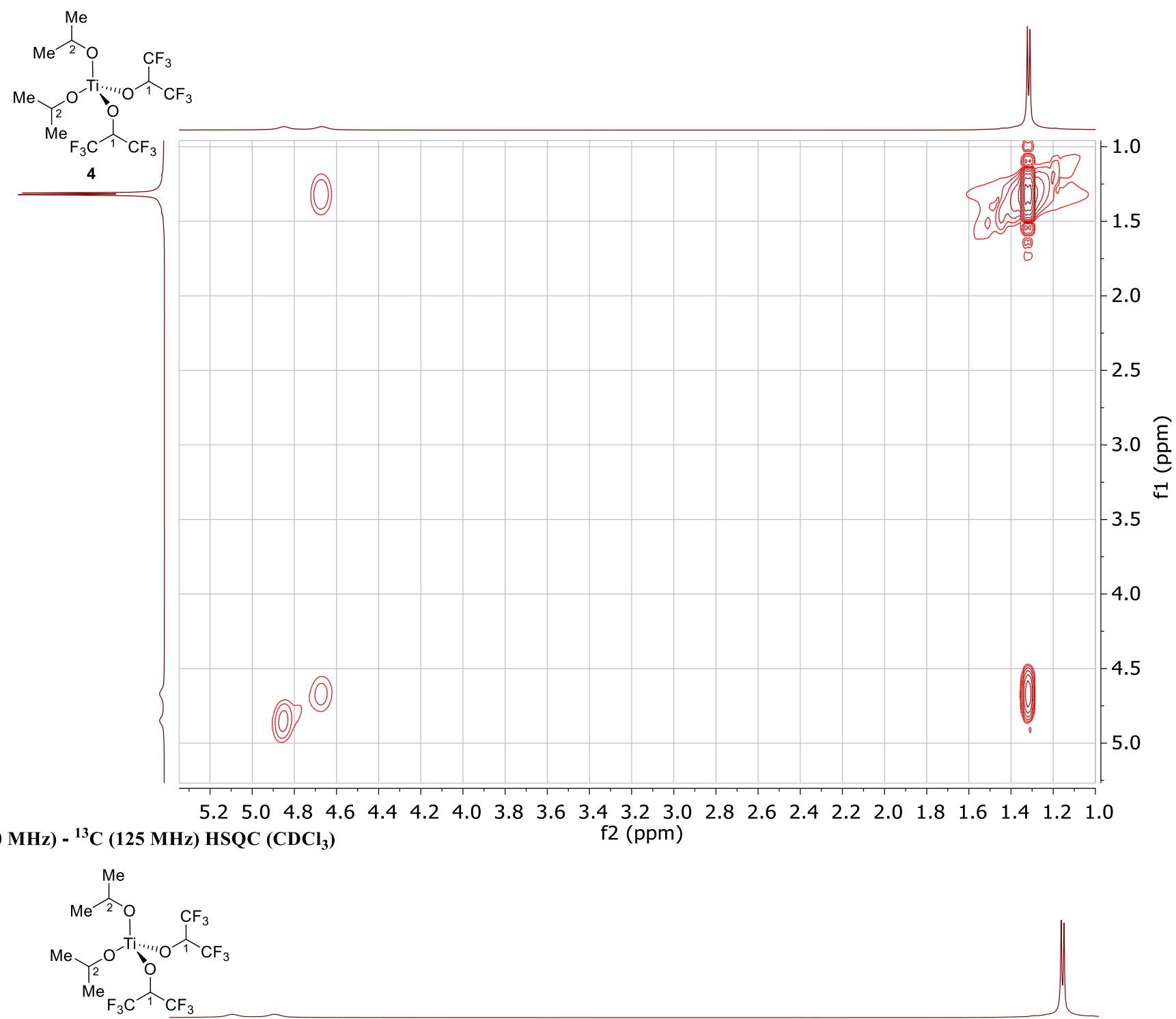

${ }^{1} \mathrm{H}(500 \mathrm{MHz})-{ }^{13} \mathrm{C}(125 \mathrm{MHz}) \mathrm{HSQC}\left(\mathrm{CDCl}_{3}\right)$

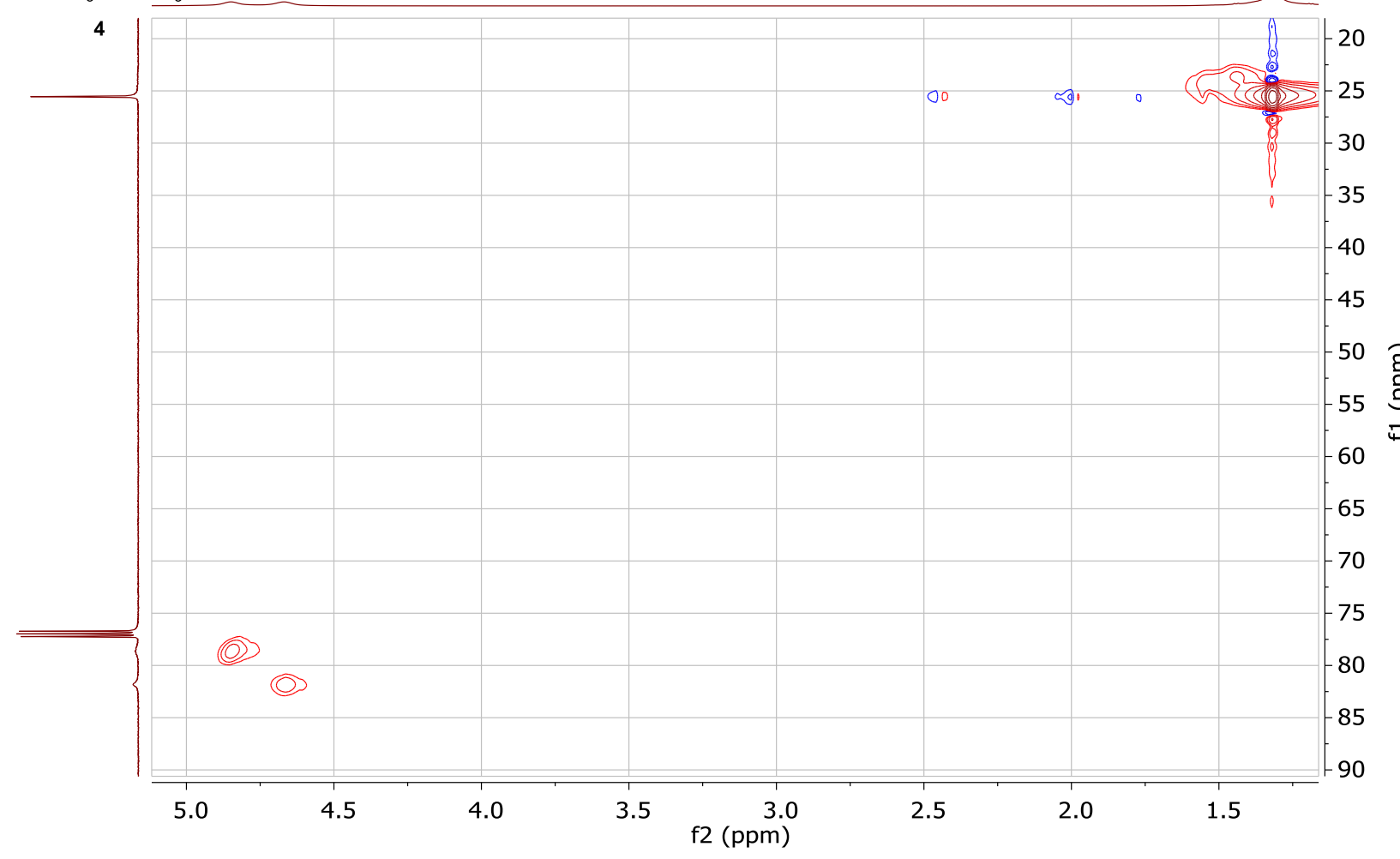


${ }^{19}$ F NMR (471 MHz, $\mathrm{CDCl}_{3}$ )
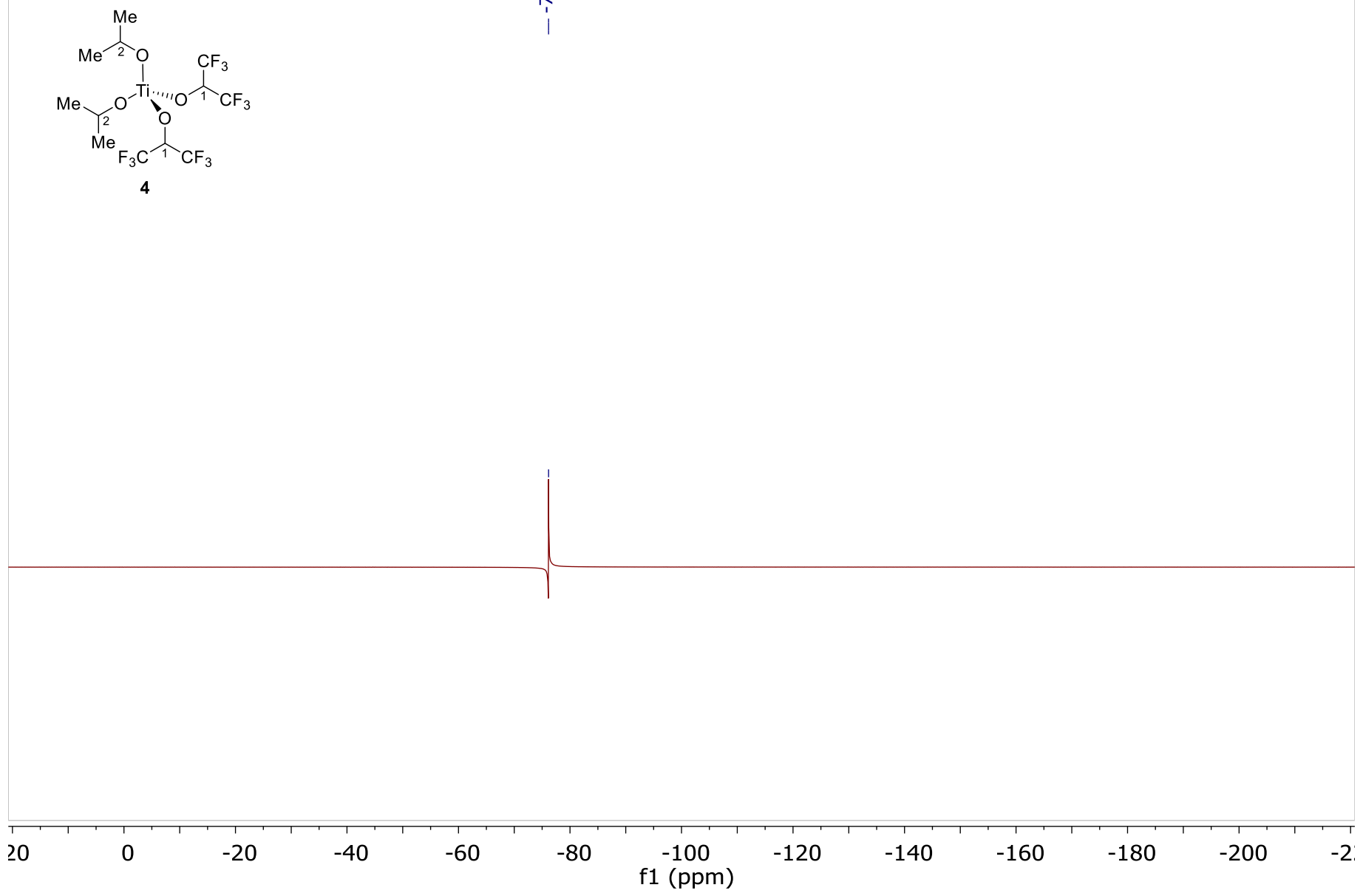

${ }^{19}$ F (471 MHz, CDCl3) - ${ }^{1} \mathrm{H}\left(500 \mathrm{MHz}, \mathrm{CDCl}_{3}\right)$ HOESY
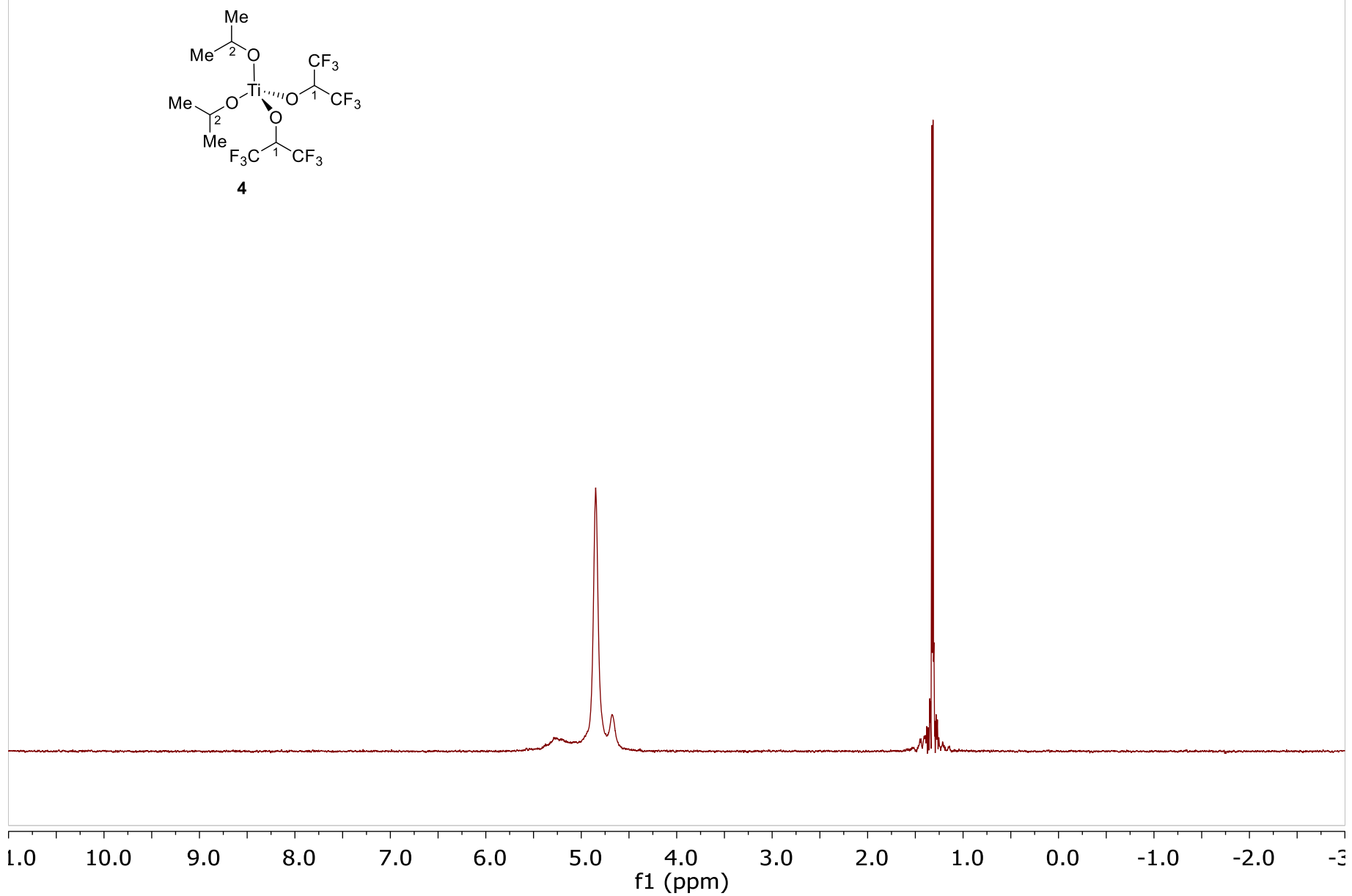
${ }^{1} \mathrm{H}$ NMR (500 MHz, $\mathrm{CDCl}_{3}$ )

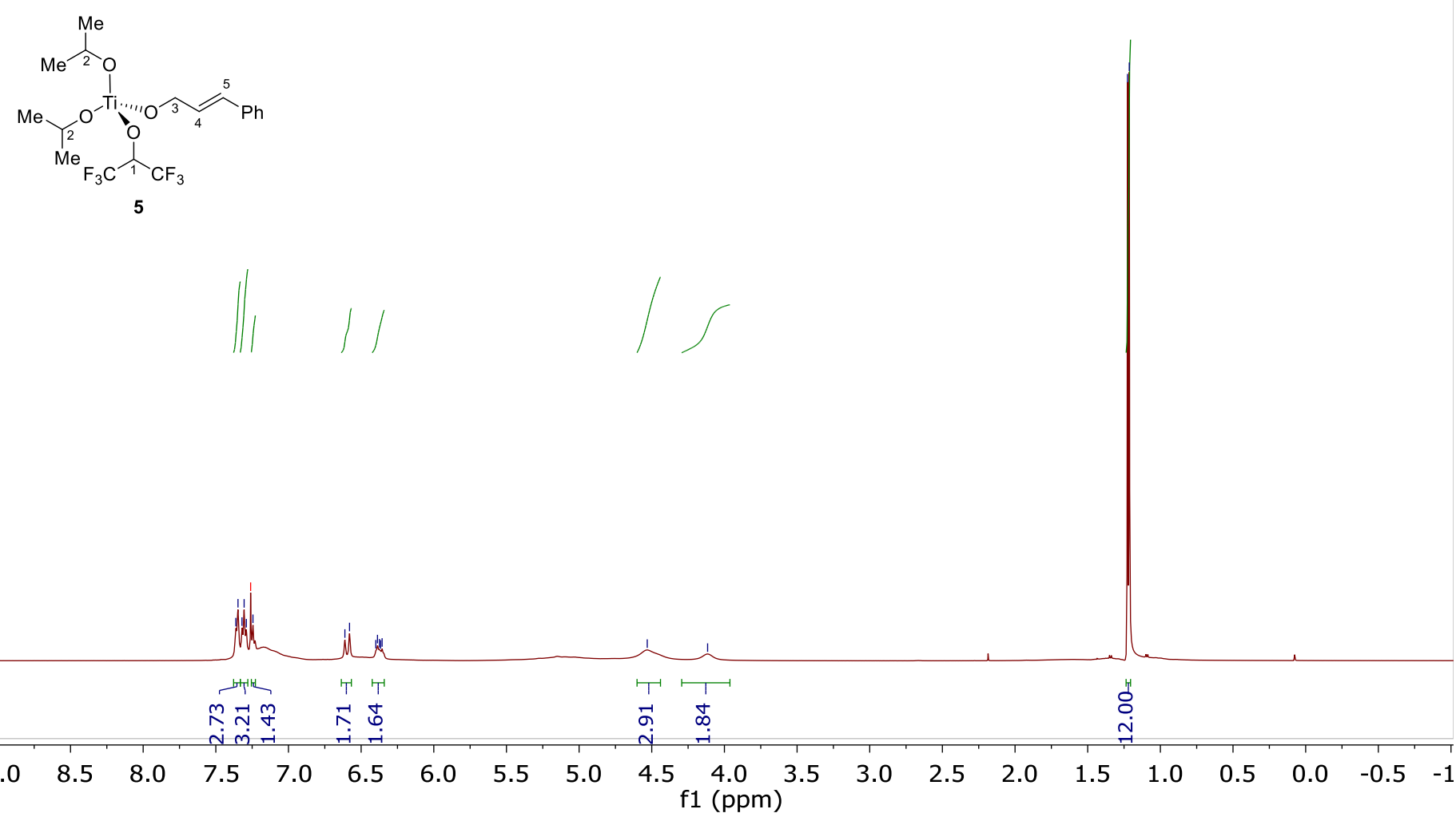

\section{${ }^{1} \mathrm{H}$ - ${ }^{1} \mathrm{H} \operatorname{COSY}-90\left(500 \mathrm{MHz}, \mathrm{CDCl}_{3}\right)$}

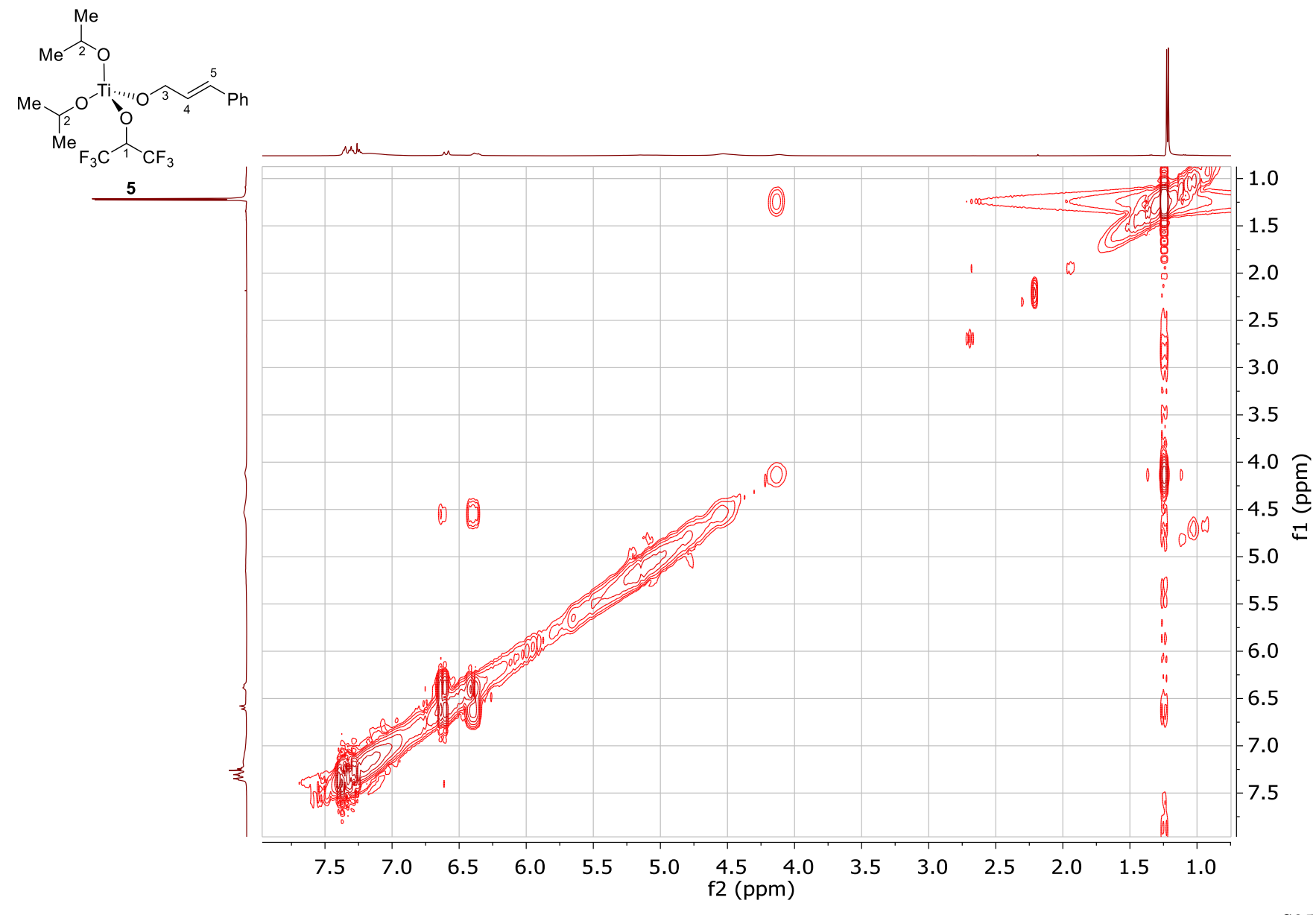




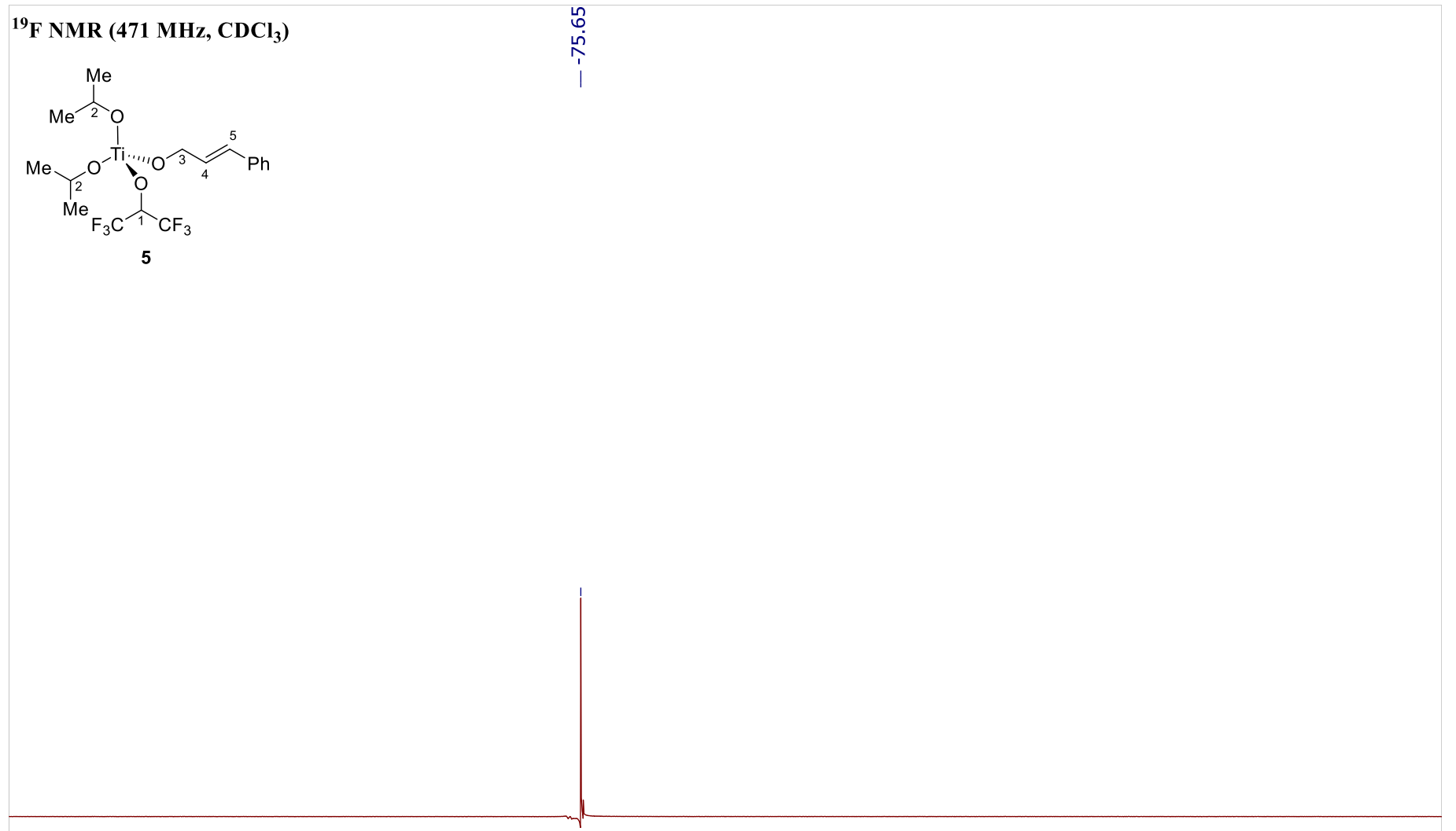

\begin{tabular}{|c|c|c|c|c|c|c|c|c|c|c|c|c|}
\hline 20 & 0 & -20 & -40 & -60 & -80 & $\begin{array}{c}-100 \\
\mathrm{f} 1(\mathrm{ppm})\end{array}$ & -120 & -140 & -160 & -180 & -200 & -2 \\
\hline
\end{tabular}

NOESY-2D (500 MHz, $\mathrm{CDCl}_{3}$ )

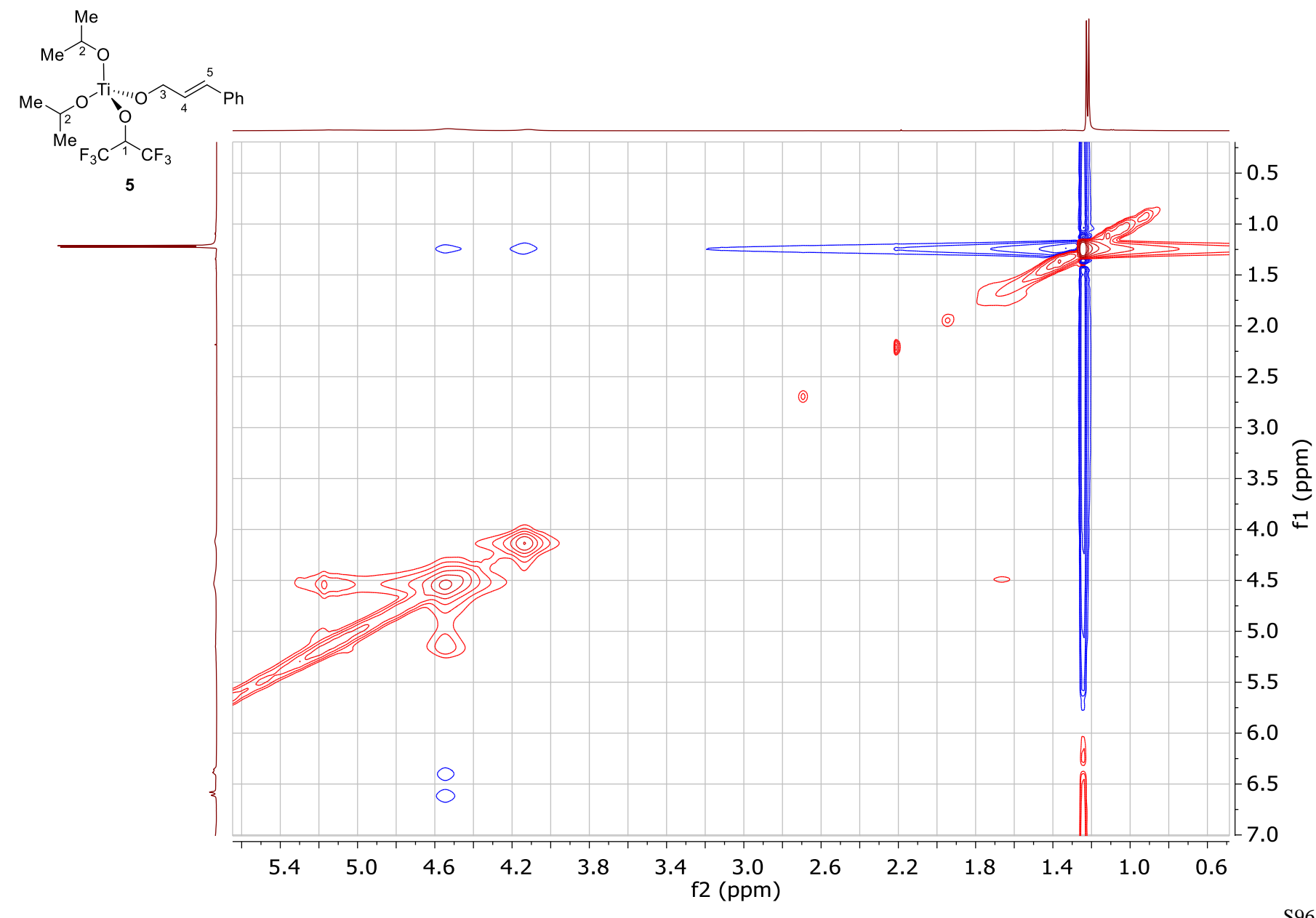

